\title{
Corneal
}

\section{Biomechanical Properties:}

Measurement, Modification and Simulation

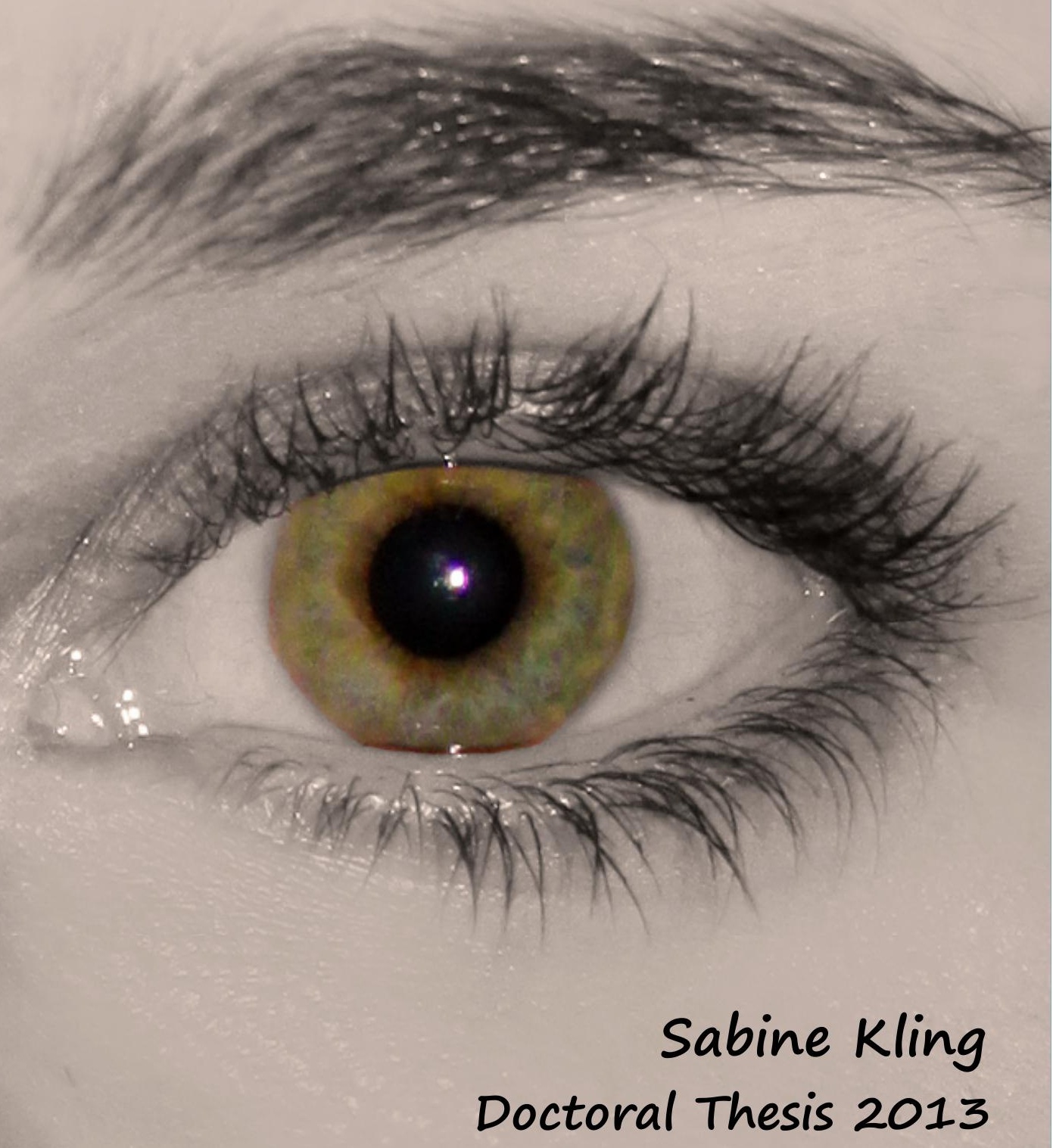

Supervisor: Susana Marcos, Instituto de Óptica, CSIC 


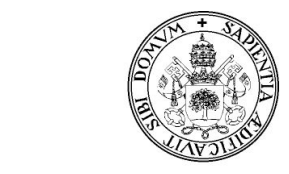

Universidad deValladolid

Impreso 2T

\section{AUTORIZACIÓN DEL DIRECTOR DE TESIS}

(Art. 2.1. c de la Normativa para la presentación y defensa de la Tesis Doctoral en la UVa)

$D^{a}$. Susana Marcos Celestino, con D.N.I. $n^{\circ} 07954600 \mathrm{G}$

profesora del departamento de Imágenes, Visión e Instrumentación óptica

Centro: Instituto de óptica “Daza de Valdés”, cSIC

como Director de la Tesis Doctoral titulada: Corneal Biomechanical Properties: Measurement, Modification and Simulation

presentada por $\mathrm{D}^{\mathrm{a}}$. Sabine Kling

alumna del programa: Doctorado en Ciencias de la Visión

impartido por el departamento de Facultad de Medicina, Instituto de Oftalmobiología Aplicada

autoriza la presentación de la misma, considerando que la tesis es original y de alto impacto, y constituye un hito importante en el conocimiento de las propiedades biomecánicas de la cornea, a través de nuevos desarrollos de instrumentación de imagen, experimentación y modelado.

Valladolid, 28 de Octubre de 2013

El Director de la Tesis,

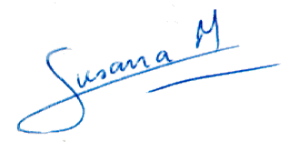

Fdo.: Susana Marcos Celestino 


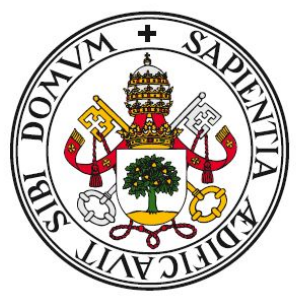

\section{Universidad deValladolid}

FACULTAD DE MEDICINA

INSTITUTO DE OFTALMOBIOLOGÍA APLICADA

\section{Corneal Biomechanical Properties:}

Measurement, Modification and Simulation

\section{DISSERTATION}

SUBMITTED IN PARTIAL FULLFILLMENT OF THE REQUIREMENTS FOR THE DEGREE OF DOCTOR OF PHILOSOPHY.

November 22, 2013

Author:

Sabine M. KLING
Supervisor:

Prof. Susana MARCos 


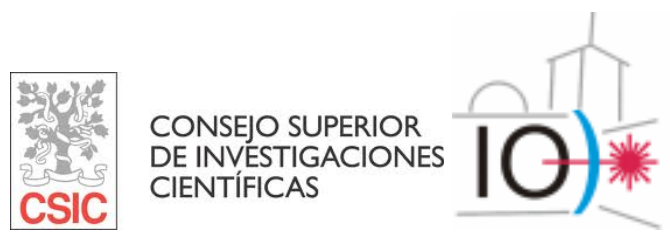




\section{Preface}

\section{Words of appreciation}

TO MY PARENTS I am grateful that you always believed in me and gave me support in everything I did. Thank you for the many emails keeping me updated and having me feel close.

TO MY SUPERVISOR A big thank you Susana, for giving me the opportunity to come to your renown research group in Madrid, for guiding me to get out the best of experiments and simulations, for teaching me the scientific language and for your constant search for new projects and collaborations, which allowed me to contribute to many national and international conferences and to be in contact with the leading groups of the field.

TO OUR COLLABORATORS Thank you Haris, for receiving me in your lab at the University of Heraklion during my short stay in 2011, for your efforts making my visit important and for getting out a nice publication. Thank you Giuliano and Andy, for receiving me in your lab at Harvard University during my short stay in 2012, for the great experience performing experiments with your novel optical systems and for the enthusiastic ambiance, which made my stay very productive.

TO MY COLLEAgUES I would like to thank all of our group members for the friendly atmosphere in the office, during lunch breaks and at congresses. I also appreciate having you insist on the Spanish language, which helped me a lot in improving my Spanish skills. The daily practice finally made it unnecessary to attend a Spanish course. Laura, thank you for helping me with many things when I arrived in Madrid for the first time. 
To RoDRIGO Thank you for the many great moments, no matter of being near or in a long distance. Travelling and discovering the world together made the time fly away, let me distract and always come up with new ideas.

To THE SPANISH GOVERNMENT I acknowledge the financial support I received in form of a FPI predoctoral fellowship. 


\section{Motivation}

The cornea is the most important optical structure in the eye and responsible for $60 \%$ of its optical power. Small alteration from its physiological state, such as irregularities in its shape and surface, induce aberrations and reduce the visual performance. Thereby the corneal shape is determined by its micro-structure and biomechanical properties. In the physiologic condition the stress-strain distribution in the cornea is equilibrated. However this equilibrium gets disturbed when corneal tissue is removed (LASIK, PRK) or material is added within the cornea (ICRS). Therefore corneal reshaping and hence the outcome of refractive surgeries depends to a large extend on the immediate biomechanical response of the cornea, but also on later ongoing wound healing effects. The structural equilibrium of the cornea gets also disturbed in certain pathologies, such as keratoconus, where the cornea suddenly becomes unstable and starts deforming non-uniformly. This induces a high amount of aberrations resulting in a continuously degrading vision. New treatments, such as collagen cross-linking, aim at increasing the corneal stiffness in order to stop the corneal steepening with keratoconus. Currently the prediction of refractive outcomes after surgery and the evaluation of the effect of cross-linking are limited by uncertainties regarding the biomechanical properties of the cornea. The corneal elasticity modulus reported in literature spans over two orders of magnitude, mostly from measurements in vitro. In vivo measurements are even more challenging. Predictive models for surgical results require accurate mechanical input parameters of the cornea. Also, corneal cross-linking can only be quantitatively evaluated by a proper assessment of the biomechanical properties of the cornea. Besides, the assessment of corneal biomechanical properties may facilitate an early diagnosis of corneal disease, such as keratoconus. This dissertation is devoted to study the corneal biomechanics combining state-of-the-art optical imaging technologies with finite element modeling. The developed methodology allowed us to quantify the biomechanical properties of the cornea and holds promise to become a much needed clinical tool in the future.

\section{Open questions addressed in this thesis}

MEASURING CORNEAL BIOMECHANICAL PROPERTIES IN A MORE PHYSIOLOGICAL STATE USING OPTICAL IMAGING TECHNIQUES Generally corneal mechanical properties are quantitatively measured by strip extensiometry 
(i.e. uni-axial stretching of flattened corneal sections). However fixation and stress application in those measurements greatly differs from the physiological condition of the cornea. On the other hand, inflation tests typically record only the displacement of the corneal apex and do not consider the entire deformation of the corneal shape. Quantitative optical imaging techniques will allow to accurately measure corneal deformation in its physiological state and under different loading conditions.

CHANGES IN CORNEAL RIGIDITY WITH UV CROSS-LINKING Cross-linking is an emerging clinical treatment to stiffen the corneal tissue. Its effect has typically been evaluated it vitro by strip-extensiometry. The mechanical properties measured with this approach might be very different from the in vivo behavior. It is important to characterize the biomechanical effect of cross-linking in order to get better understand the treatment mechanisms as well as to assess its efficacy under different treatment protocols.

MEASURING CORNEAL BIOMECHANICAL PROPERTIES BY NON-CONTACT TECHNIQUES While techniques to extract mechanical properties in vitro are extremely useful in experimental investigations, undoubtedly clinical applications require the development of in vivo techniques, which will be essential for the early diagnosis of keratoconus, to identify patients with potential risk of ectasia before performing corneal refractive surgery, and to evaluate the outcomes of treatments such as cross-linking. To date, the only wide spread commercial instrument attempting the measurement of mechanical properties is the Ocular Response Analyzer (Reichert Technologies, Depew, NY, USA), with limitations arising from the indirect nature of the measurement. Techniques relying on non-contact corneal deformation measurements, or relating microscopic structural properties with macroscopic properties are promising for in vivo measurements in the clinic.

RETRIEVING CORNEAL BIOMECHANICAL PROPERTIES FROM EXPERIMENTAL DATA FROM NEW TECHNIQUES New non-contact imaging techniques allow to measure the corneal in vivo response under different loading conditions such as air-puff ejection or vibration. The sensitivity of biomechanical properties to these loading conditions has not been studied yet. 
INCREASE PREDICTABILITY OF THE CORNEAL TREATMENTS FROM CORNEAL BIOMECHANICAL MODELING In order to relate the geometrical deformations observed under air-puff or vibration loading conditions, inverse modelling will allow retrieving the biomechanical parameters of the cornea. in vivo deformation data of patients then might be used as input in order to predict the corneal response following different surgeries (such as LASIK, PRK, intrastromal ring implantation, corneal transplantation).

\section{Goals of this thesis}

To develop new technologies to estimate corneal biomechanical properties and to apply those technologies to evaluate corneal response to corneal treatments.

In particular:

- To develop whole eye/ corneal inflation methods to obtain stressstrain responses to increased and decreased IOP

- To develop 2D extensiometry to obtain corneal elasticity of corneal flaps

- To develop an air-puff technique combined with optical coherence tomography (OCT) to measure dynamic corneal deformation noninvasive.

- To study the factors that contribute to air puff corneal deformation, including corneal geometry, IOP, the presence of sclera and in vivo or in vitro conditions

- To study the effect of hydration and storage on the corneal mechanical response to inflation.

- To perform a finite element analysis of inflation models to estimate the biomechanical properties of the cornea.

- To develop a finite element modeling of air puff corneal deformation to obtain the biomechanical properties of the cornea.

- To develop a finite element model of OCT vibrography.

- To evaluate changes in the biomechanical properties of the cornea produced by cross-linking from inflation experiments, 2D-flap extensiometry, corneal deformation imaging following an air-puff and OCT-vibrography. 
- To evaluate local changes in stiffness produced by standard crosslinking and transepithelial cross-linking from Brillouin microscopy.

- To analyze the corneal mechanical response and refractive changes to ICRS in normal and central keratoconic corneas using finite element modelling.

\section{Hypothesis}

Advanced imaging technologies in combination with sophisticated analytical tools will allow estimates of the corneal biomechanical properties and its response to treatment.

\section{Structure of this dissertation}

This thesis addresses the measurement of the corneal biomechanical properties. Techniques were developed to measure the corneal stiffness in vitro in order to study the corneal behavior as a function of different factors (such as hydration, geometry, intraocular pressure, corneal stiffness). Experimental data were used to build numerical models, which were able to reproduce the observed biomechanical response of the cornea. Numerical models were applied to retrieve biomechanical parameters from in vivo deformation measurements. Numerical models were also applied to study the outcome with implantation of intrastromal ring segments. The dissertation is structured as follows:

- Chapter 1 The thesis addresses physiological, medical, optical and mechanical aspect of the cornea. This chapter gives an overview of relevant principles and methods, including the physiology of the cornea, common corneal pathologies, optical scattering and interference theory, mechanical material testing and numerical material models.

- Chapter 2 This chapter describes the experimental and theoretical materials and methods applied in this thesis, including optical imaging systems, eye handling, corneal treatments, intraocular pressure control system, analysis routines of experimental data, mechanical finite element simulation and optical ray tracing analysis.

- Chapter 3 The corneal response to variable intraocular pressure (IOP) was studied in untreated porcine eyes and after UV collagen 
cross-linking (CXL). Enucleated eyes were treated with standard CXL conditions in vitro and contralateral eyes served as control. Eyes were measured by Scheimpug corneal three-dimensional topographer. Images were obtained while the IOP either remained constant or varied. Immediately after enucleation and 24 hours after, corneal geometry was analyzed as a function of IOP, and whole globe stress-strain curves were calculated. We found that cross-linked corneas deformed less than un-treated corneas and had a larger Young's modulus.

- Chapter 4 Corneal deformation under intraocular pressure variation was measured as a function of corneal hydration state, modulated by the storage solutions or postmortem period. Fresh enucleated porcine eyes were used for in vitro whole eye globe inflation experiments. We found that hydration and storage solutions significantly affected the biomechanical response.

- Chapter 5 Corneal biomechanical properties are usually measured by strip extensiometry or inflation methods. We developed a two-dimensional (2D) flap extensiometry technique, combining the advantages of both methods, and applied it to measure the effect of UV-Riboflavin cross-linking (CXL). Corneal flaps from the anterior stroma were mounted on a custom chamber filled with Riboflavin and silicone oil. Changes in refractive power with inflation were monitored using a ray-tracing aberrometer. Young's modulus was estimated from analytical modelling. Confocal microscopy examination was performed before and at different times after CXL. We quantified the stiffness increase in the anterior stroma 1-month post CXL.

- Chapter 6 Brillouin microscopy allows the local estimation of corneal elasticity with high lateral and axial resolution. We validated noncontact Brillouin microscopy to quantify corneal mechanical properties before and after CXL. CXL was performed on fresh porcine eyes using various presoaking times and light doses, with or without epithelial debridement. From Brillouin maps of corneal elastic modulus, stiffness and average modulus of anterior, middle, and posterior stroma were analyzed. Corneal stiffening index (CSI) was introduced as a metric to compare the mechanical efficacy of a given CXL protocol with respect to the standard protocol. The irradiation time was more important than the pre-soaking time. The epi-on protocol was less effective than the standard protocol. 
- Chapter 7 A new technique is presented for the non-invasive imaging of the dynamic response of the cornea to an air puff inducing a deformation. A high-speed spectral OCT instrument combined with an air tonometer in a non-collinear configuration was used to image the corneal deformation over full corneal cross-sections, as well as to obtain high speed measurements of the temporal evolution of the corneal apex. A quantitative analysis allowed direct extraction of several deformation parameters, such as amplitude, diameter and volume of the maximum deformation, as well as duration and speed of the increasing deformation period and the recovery period. The potential of the technique is demonstrated on porcine corneas in vitro under constant IOP for several conditions (untreated, after riboflavin instillation and under cross-linking with ultraviolet light), as well as on human corneas in vivo. A significant difference in the corneal deformation pattern was observed after cross-linking and between in vivo and in vitro measurements.

- Chapter 8 Dynamic corneal deformation imaging systems based on Scheimplug imaging have been recently introduced commercially. In this study we tested the influence of several factors on corneal deformation to an air-puff: Intraocular Pressure (IOP), corneal rigidity, dehydration, presence of sclera, and in vivo versus in vitro conditions. Porcine and human donor eyes were used for in vitro experiments and human volunteers were measured in vivo (normal and cross-linked corneas). The temporal corneal deformation was characterized by the apex indentation across time, the maximal indentation depth and the temporal symmetry (comparing inward versus outward deformation). The spatial corneal profile was characterized by the peak-distance at maximal deformation. The deformation pattern varied significantly across conditions, with cross-linked corneas showing a smaller and temporally more symmetric deformation.

- Chapter 9 In this study a finite element model was built to reproduce experimental data of the corneal response to an air-puff. We simulated different stiffness, hydration and boundaries to prove the adaptability of the numerical model to experimental data. A sensitivity analysis was performed for the human in vivo condition to study the correlation between geometrical deformation of the cornea and its biomechanical parameters. Our results show that dynamic imaging gives access to inherent material proper- 
ties and demonstrate the importance of dynamic properties in the mechanical characterization of soft tissues.

- Chapter 10 OCT vibrography is a novel technique based on resonance to assess the corneal biomechanical properties by noncontact. We demonstrated the ability of OCT vibrography to determine corneal material parameters, while reducing current prevalent restrictions. Numerical simulations were performed using an axissymmetric finite element (FE) model to demonstrate the expected dependency of the frequency response curve on corneal biomechanical parameters in isolated thin corneal membranes and eye globes. The effect on the corneal natural frequencies of the following parameters was studied: elastic modulus, viscoelasticity, geometry (thickness, curvature), intraocular pressure and density. The model was used to predict the biomechanical response in flaps from bovine corneas and in enucleated porcine eyes. Sound excitation together with a phase-sensitive OCT was used to measure the frequency response curve in anterior and posterior corneal flaps, and in virgin and UV-cross-linked whole globes in order to determine the effect of different corneal rigidity. A shift to higher natural frequencies occur as a result of an in increase in corneal stiffness.

- Chapter 11 Intrastromal corneal-ring segments (ICRSs) are applied to improve the symmetry and to modify the shape of highly distorted corneas in keratoconic, myopic, and astigmatic patients. For the current surgical treatment ICRS geometry and position are primarily chosen based on empirical nomograms. We developed a finite-element model (FEM) to predict the corneal response to different ICRS geometries in normal and central keratoconic corneas. Ocular tissues (cornea, limbus, sclera) were simulated by a hyperelastic material and the ICRS by a linear elastic material (polymethyl-methacrylate). An incrustation procedure was performed to consider the local material addition, the rigidity increase at the ICRS position and the geometric shape (triangular, hexagonal) of the ICRS. We studied the effect of different ICRS heights and optical zones in normal and axis-symmetric keratoconic corneas. The simulation results confirm clinical trends that a smaller optical zone and a thicker ICRS are most effective. Using patient specific geometric and biomechanical input data will allow a better individual prediction of ICRS implantation in the future.

- Chapter 12 This chapter summarizes the conclusions of the dif- 
ferent studies assembled in this dissertation. We report a steeper stress-strain curve of cross-linked than virgin corneas in whole globe inflation tests. Also storage media and hydration state were found to have a significant effect on the corneal biomechanical response. A 2D-flap extensiometry technique was developed that provided the natural bi-directional stress distribution. We revealed differences in the corneal stiffening pattern for different cross-linking protocols by Brillouin microscopy. We found that the geometrical corneal deformation following an air-puff depends on corneal stiffness, thickness, intraocular pressure and the presence of the sclera. Thereby the experimental data could be well reproduced with a finite element model. We propose a novel vibration technique to measure corneal dynamics by non-contact and provide supporting simulation and experimental data. We also predict the refractive change after implanting intrastromal ring segments with numerical simulation.

- Chapter 13 This chapter lists the publications and congress contributions that arose out of the work of this thesis.

- Chapter 14 Resúmenes en Español 


\section{Contents}

Preface

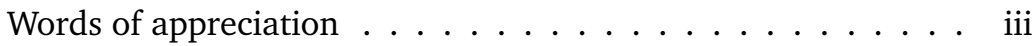

Motivation .....................

Open questions addressed in this thesis ........

Goals of this thesis . . . . . . . . . . . . . . vii

Hypothesis . . . . . . . . . . . . . . viii

Structure of this dissertation $\ldots \ldots \ldots$. . . . . . viii

1 Introduction $\quad 1$

Physiological background . . . . . . . . . . . . . 1

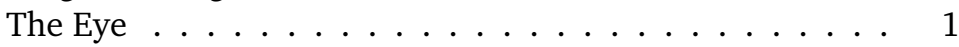

The Cornea . . . . . . . . . . . . . . 3

Layers of the Cornea . . . . . . . . . . . 4

Refractive Power . . . . . . . . . . 7

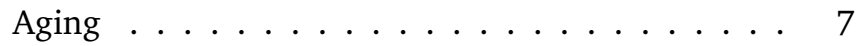

Optical background $\ldots \ldots \ldots \ldots \ldots$

Wavefront .................. 8

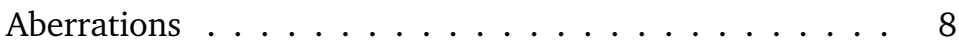

Refractive Errors of the Eye . . . . . . . . . 9

Ray-tracing system . . . . . . . . . . . . . . 11

Coherence . . . . . . . . . . . . . 12

Interference . . . . . . . . . . . . . . 13

Anterior segment imaging techniques . . . . . . . . . 13

Scheimpflug Imaging . . . . . . . . . . . . . 13

Optical Coherence Tomography . . . . . . . . . . 14

Ultrasonic Imaging . . . . . . . . . . . . . . 16

Mircroscopic imaging of the cornea . . . . . . . . . . . 17

X-ray scattering . . . . . . . . . . . . . 17

Second harmonic generation microscopy . . . . . . 19

Brillouin microscopy . . . . . . . . . . . . 20 
Corneal disease and treatment background . . . . . . . . . 21

Corneal Pathologies . . . . . . . . . . . . . . . 21

Keratoconus . . . . . . . . . . . 21

Corneal Treatments . . . . . . . . . . . . . . . 22

UV Corneal Collagen Cross-linking . . . . . . . . . 22

Intrastromal Corneal Ring Segments . . . . . . . 25

Mechanical background . . . . . . . . . . . 26

Finite Element Method . . . . . . . . . . . . . 26

FEM Analysis in Ansys . . . . . . . . . . . . . . 27

Material Models . . . . . . . . . . . . . . . . . 28

Elasticity . . . . . . . . . . . . 29

Viscoelasticity . . . . . . . . . . . . 30

Hyperelasticity . . . . . . . . . . . . . 32

Orthotropic Properties . . . . . . . . . . . . 33

Anisotropic Properties . . . . . . . . . . . . 34

Models for the Cornea . . . . . . . . . . . . . . . 34

Simple Analytical Approach . . . . . . . . . . . 34

Complex Analytical and Numerical Approaches . . 35

Material Testing . . . . . . . . . . . . 36

Stress-Strain Test . . . . . . . . . . . . 36

Modal Testing . . . . . . . . . . . . . . 38

Dynamic Modulus . . . . . . . . . . . 39

Measuring Corneal Elasticity . . . . . . . . . . . . 40

in vitro Methods . . . . . . . . . . . . . . 40

1D-Strip Extensionmetry . . . . . . . . . . . 40

Button Inflation . . . . . . . . . . . . . . . 41

Whole-Globe Inflation . . . . . . . . . . . . 42

Clinically applied in vivo methods . . . . . . . . . . . . . 42

Eye inflation . . . . . . . . . . . . . . 42

Ocular Response Analyzer . . . . . . . . . . . . . 43

Air-puff with Scheimpflug . . . . . . . . . . . . 45

State of the art of experimental ocular biomechanics . . . 45

Cornea ................ 4 45

Sclera .................. 47

2 Methods $\quad 49$

Experimental Methods . . . . . . . . . . . . . . 51

Imaging Systems . . . . . . . . . . . . . 51

Scheimpflug Imaging . . . . . . . . . . . . . . . . 51

Optical Coherence Tomography . . . . . . . . . 51

Ray Tracing Aberrometry . . . . . . . . . . . 56 
Brillouin Microscopy . . . . . . . . . . . . . . 57

Air-puff Systems . . . . . . . . . . . . . . . 57

Pressure System . . . . . . . . . . . . . . . 60

Wet-Chamber ................. 61

Eye-Holders . . . . . . . . . . . . . . . . . . 62

Whole Eye Globes . . . . . . . . . . . . . 62

Corneal Buttons . . . . . . . . . . . . 63

2D-Flap Holder . . . . . . . . . . . . . . 63

Microkeratome ............... 65

Eyes ........................... 66

Porcine Eyes . . . . . . . . . . . . 666

Rabbit Eyes . . . . . . . . . . . . . . . . 666

Human Eyes . . . . . . . . . . . . . . 66

Storage .................. 66

Corneal Treatments . . . . . . . . . . . . . . . 67

UV-Collagen Cross-linking . . . . . . . . . . 67

Hydration Conditions . . . . . . . . . . . . 68

Analysis Routines . . . . . . . . . . . . . . . . 69

Air-puff Deformation . . . . . . . . . . . . 69

2D-Flap Geometry . . . . . . . . . . . . . . 72

Simple Inflation Model . . . . . . . . . . . . 73

Corneal Stiffening Index . . . . . . . . . . . . . . . 74

Theoretical Methods . . . . . . . . . . . . . . . 75

Software . . . . . . . . . . . . . 75

Matlab . . . . . . . . . . . 75

ANSYS APDL . . . . . . . . . . . 76

ZEMAX ................. 76

Refractive Analysis of FE-models . . . . . . . . . . 76

Corneal Refraction . . . . . . . . . . 76

Spherical Aberration . . . . . . . . . . . . . 77

Finite Element Model . . . . . . . . . . . . . . . . . 77

Air-puff FEM . . . . . . . . . . . . . . 77

Vibrography FEM . . . . . . . . . . . . . . . . . . . . . . . . . . 78

ICRS FEM . . . . . . . . . . . . . . 78

FEM Solver . . . . . . . . . . . . . . 78

Sensitivity Analysis . . . . . . . . . . . . 78

3 Biomechanical Changes after Collagen Cross-Linking from $\begin{array}{ll}\text { Porcine Eye Inflation Experiments } & 79\end{array}$ Introduction . . . . . . . . . . . . . . 81 
Protocols ..................... 83

Set-up ..................... 84

Data analysis . . . . . . . . . . . . . 85

Results .................. 86

Changes in corneal thickness . . . . . . . . . 86

Changes in the corneal apex position ... . . . . 87

Change in horizontal and vertical corneal radius of curvature 90

Refractive changes: varying versus constant pressure . . . 94

Simple Biomechanical Model: Young's Modulus and Hys-

teresis . . . . . . . . . . . . 9 97

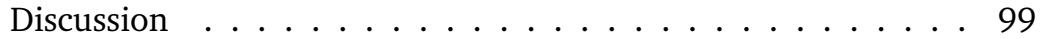

Comparison to previous clinical and experimental data . . 99

Implications of the results . . . . . . . . . . . . 102

4 Effect of hydration state and storage media on corneal biomechanical response from in vitro inflation tests 105 Introduction . . . . . . . . . . . . . . 107

Conditions and Protocols . . . . . . . . . . . . . . 108

Results . . . . . . . . . . . . . . . 110

Corneal Thickness . . . . . . . . . . . . . . . 110

Corneal Curvature . . . . . . . . . . . . . . 111

Corneal Hydration . . . . . . . . . . . . . . . 113

Epithelium and Corneal Transparency . . . . . . . . . 113

Discussion . . . . . . . . . . . . . . . . 114

5 Corneal biomechanical properties from two-dimensional corneal flap extensiometry: application to UV-Riboflavin $\begin{array}{lr}\text { cross-linking. } & 119\end{array}$

Introduction . . . . . . . . . . . . . . . . . . 121

Protocols . . . . . . . . . . . . . . . 122

Stress-strain cycles . . . . . . . . . . . . . . . . . 124

Data Analysis . . . . . . . . . . . . . . . . 124

Results . . . . . . . . . . . . . . . 125

Flap Pachymetric and Microscopic Observations . . . . . . 125

Defocus Aberration . . . . . . . . . . . . . . . 125

Asitgamtic Aberration . . . . . . . . . . . . . 128

Young's Modulus . . . . . . . . . . . . . . . 128

Discussion . . . . . . . . . . . . . . . . 129 
6 Brillouin microscopy of collagen cross-linking: non-contact depth-dependent analysis of corneal elastic modulus

Introduction . . . . . . . . . . . . . 135

Protocol . . . . . . . . . . . . . . 136

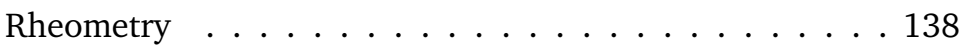

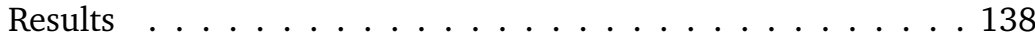

In situ mechanical characterization of corneas after standard CXL . . . . . . . . . . . . . . 138

Effect of pre-soaking time and UV light exposure time . . . 140

Corneal collagen cross-linking without epithelial debridement .................. . . 142

Sham controls . . . . . . . . . . . . . . . . . . . 144

Hydration effects on Brillouin modulus and shear modulus 145 Discussion ................... 146

7 Determination of corneal biomechanical properties from deformation following an air-puff

Introduction . . . . . . . . . . . . . . . 153

Protocol . . . . . . . . . . . . . . 156

Calibration and validation . . . . . . . . 156

Eyes and measurements . . . . . . . . . . . . . 157

Results

158

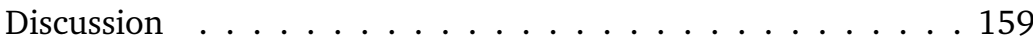

Conclusions . . . . . . . . . . . . . . 163

8 Contributing factors to corneal deformation in air-puff measurements $\quad 165$ Introduction . . . . . . . . . . . . . . . 167

Conditions and Protocols . . . . . . . . . . . . 169

Pig eyes . . . . . . . . . . . . . . . 169

Human eyes . . . . . . . . . . . . . . . . 170

Different IOPs (data set 1 ) . . . . . . . . . . . . . 170

Corneal thickness and hydration state (data set 1 ) . . . . 170

Corneal rigidity (data set 1 ) . . . . . . . . . . . . 171

Corneal buttons versus whole eyes (data set 2) . . . . . 171

in vitro versus in-vivo (data set 3 ) . . . . . . . . . . . 171

Analysis . . . . . . . . . . . . . . . . 172

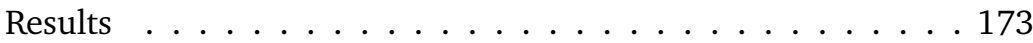

Effect of intraocular pressure (IOP) - data set $1 \ldots 173$

Spatial deformation profile . . . . . . . . . . 174 
Effect of dehydration and CXL - data set $1 \ldots 174$

Temporal apex deformation . . . . . . . . . 174

Spatial deformation profile . . . . . . . . . 175

Difference between button and whole globe - data set 2.176

Temporal apex deformation . . . . . . . . . 176

Spatial deformation profile . . . . . . . . 177

Difference between in vitro and in-vivo - data set 3 . . 178

Temporal apex deformation . . . . . . . . 178

Spatial deformation profile . . . . . . . . . 178

Central corneal thickness - all data sets . . . . . . . . . 178

Repeatability of measurements - data set $2 \ldots \ldots 178$

Discussion . . . . . . . . . . . . . . . . 179

9 Non-invasive in vivo evaluation of corneal viscoelastic biome$\begin{array}{ll}\text { chanical parameters } & 185\end{array}$

Introduction . . . . . . . . . . . . . . 187

Eye Modelling . . . . . . . . . . . . . . . . 190

Air-puff . . . . . . . . . . . . . . . . . 191

Different corneal conditions . . . . . . . . . . . . 191

Different intraocular pressures (IOPs) . . . . . 191

Different boundary conditions . . . . . . . . . . . . 193

What determines the corneal response? . . . . . . . . . 194

Discussion and conclusions . . . . . . . . . . . . 195

Methods . . . . . . . . . . . . . . . . . 199

Air Puff Imaging . . . . . . . . . . . . . . . . 199

Subjects and experimental data . . . . . . . . . . . 199

Air-puff characterization . . . . . . . . . . . . . . 199

Finite element simulation . . . . . . . . . . 200

Geometry . . . . . . . . . . . . . 200

Material models . . . . . . . . . . . 200

Boundary conditions and loads . . . . . . . . . 201

Air-puff application . . . . . . . . . . . . . 201

Ocular muscle contribution . . . . . . . . . . 201

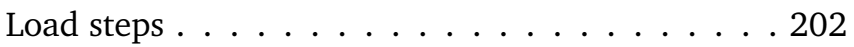

Parameter selection . . . . . . . . . . . . . 202

Analysis . . . . . . . . . . . . . . . . 202

Computing techniques . . . . . . . . . . 203

10 Non-invasive quantification of corneal biomechanical properties using OCT-Vibrography 205 Introduction . . . . . . . . . . . . . 207 
Protocol . . . . . . . . . . . . . . . . 208

Finite element (FE) simulation . . . . . . . . . . . 208

Geometry and boundaries . . . . . . . . . . . 209

Material model and biomechanical properties . . . 209

Analysis . . . . . . . . . . . . . . 210

Experiments ..................... 210

Phase-sensitive OCT . . . . . . . . . . . 211

Experimental protocol . . . . . . . . . . . 211

Eyes / conditions . . . . . . . . . . . . 211

Data analysis . . . . . . . . . . . . . 212

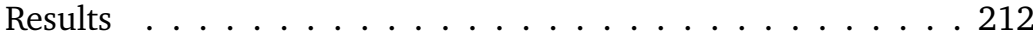

Biomechanical properties . . . . . . . . . . . 212

Corneal flaps . . . . . . . . . . . . . . . . 212

Whole eye globes . . . . . . . . . . . . . . . . 217

Discussion . . . . . . . . . . . . . 223

11 Finite-Element Modeling of Intrastromal Ring Segment Implantation into a Hyperelastic Cornea 227

Introduction . . . . . . . . . . . . . . . . . 229

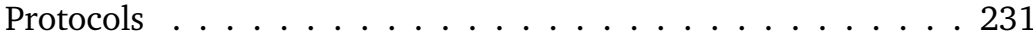

Simulation of ICRS implantation . . . . . . . . . . 231

FE Modeling of ICRSs . . . . . . . . . . . . . . . 232

Triangular Cross-Sectional ICRSs . . . . . . . . . 233

Hexagonal Cross-Sectional ICRSs . . . . . . . . . 233

Model Description . . . . . . . . . . . . . . . 233

Simulation . . . . . . . . . . . . . 233

Keratoconic Model . . . . . . . . . . . . . 235

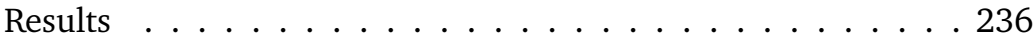

Changes in Corneal Thickness . . . . . . . . . . . 237

Effect of ICRS Geometry on Anterior Corneal Curvature . . 238

Normal Corneas . . . . . . . . . . . . 238

Keratoconic Corneas . . . . . . . . . . . . . 240

Refractive Changes . . . . . . . . . . . . . . . 241

Changes in Spherical Aberration . . . . . . . . . . . . . 242

Normal Corneas . . . . . . . . . . . . . . . 242

Keratoconic Corneas . . . . . . . . . . . . . . . 242

Effect of Keratoconus Size (Axis-Symmetric Model) . . . . 243

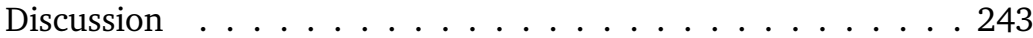

12 Conclusions $\quad 249$

Achievements . . . . . . . . . . . . . . . . . 249 
Conclusions . . . . . . . . . . . . . . . 251

Future Work . . . . . . . . . . . . . 255

13 Publications out of this thesis 257

Publications included in this thesis . . . . . . . . . . 257

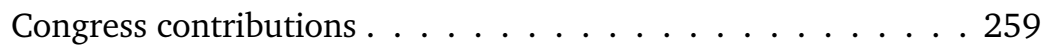

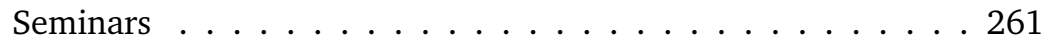

14 Resúmenes en Español 263

Capítulo $1 \ldots \ldots \ldots \ldots$. . . . . . . . . . . . . . . . . . . . . . . . . . .

Capítulo $2 \ldots \ldots \ldots \ldots \ldots$

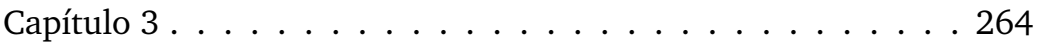

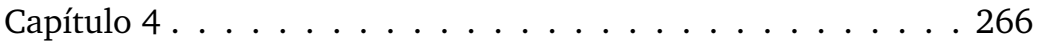

Capítulo $5 \ldots \ldots \ldots \ldots$. . . . . . . . . . . . . . . . . . . . . . . . . . . . . . . . . .

Capítulo 6. . . . . . . . . . . . . . . . 268

Capítulo $7 \ldots \ldots \ldots$. . . . . . . . . . . . . . . . . . . . . . . . . . . . . . . . . . . . . .

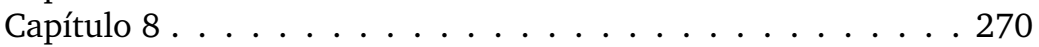

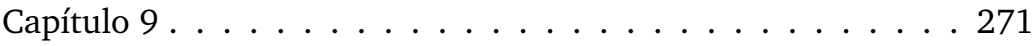

Capítulo $10 \ldots \ldots \ldots \ldots$. . . . . . . . . . . . . . . . . . . . . . . . . .

Capítulo $11 \ldots \ldots \ldots \ldots$. . . . . . . . . . . . . . . . . . . . . . . . . . . . . . . . . . .

Conclusiones en Español . . . . . . . . . . . . . . . . 274

$\begin{array}{ll}\text { Bibliography } & 293\end{array}$ 


\section{1}

Introduction

\section{Physiological background}

\section{The Eye}

As a natural optical system, the eye plays an essential role in projecting visual information from the environment into the brain. While the cornea and lens focus the incoming light to the back of the eye, photoreceptors situated in the retina absorb and convert it into chemical and electrical signals. These visual signals then get initially processed by the neural retina and transmitted by the optic nerve into the visual cortex for further processing and final perception of the visual information.

The overall ocular refractive power of the eye is around 60 Diopters, which come from the cornea and the crystalline lens. The cornea is the 
Chapter 1. Introduction

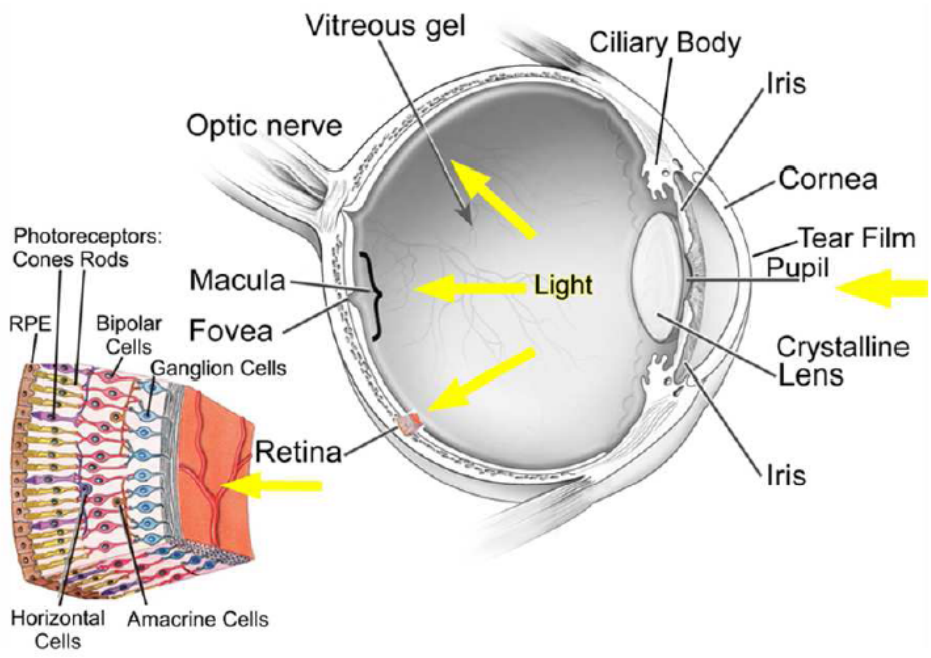

Figure 1.1: The eye, taken from Fuensanta et al [212].

main refractive component (40-43 Diopters) of the eye. In the young eye the crystalline lens has the capability to reshape upon an accommodating stimulus and contributes with a dioptric power between around $14 \mathrm{D}$ (relaxed accommodation) to $37 \mathrm{D}$ (maximally accommodated) [96]. The flexibility of the lens decreases with age and hence the ability of accommodation is lost (known as Presbyopia). A variable aperture (the iris) is placed between the cornea and the lens, contracting upon increased illumination. This aperture (pupil) controls the amount of light that enters the eye and plays a significant role in image quality.

The medium between cornea and lens is called the aqueous humor (anterior chamber), the medium between lens and retina vitreous humor. Both humors consist primarily of $\mathrm{H} 2 \mathrm{O}$ and provide the necessary pressure to maintain the shape of the ocular coat (cornea, limbus, sclera) and the lens position.

Typical dimensions of the human eye globe are: 21 - $27 \mathrm{~mm}$ axial length, $23 \mathrm{~mm}$ vertical diameter, $23.5 \mathrm{~mm}$ horizontal diameter. Typical dimensions for the human cornea are: $11 \mathrm{~mm}$ vertical diameter, $12 \mathrm{~mm}$ 
horizontal diameter, $7.86 \mathrm{~mm}$ anterior and $6.7 \mathrm{~mm}$ posterior radius of curvature.

While artificial optical systems typically have a very good on-axis optical quality, their off-axis quality is poor. In contrast the eye provides a better out-balanced system, with a more homogenous quality on- and off-axis [141].

\section{The Cornea}

The cornea forms the outer shell of the anterior eye ball with highly organized collagen fibrils, which gives the avascular tissue a high transparency (80-90\% transmission for wavelengths from $450 \mathrm{~nm}$ to $600 \mathrm{~nm}$ and even $95 \%$ to $98 \%$ for wavelengths from $600 \mathrm{~nm}$ to $1000 \mathrm{~nm}$ [12]). Both, the diameter of and the distance between individual collagen fibers is less than half of the wavelength of visible light, and therefore scattering on individual fibers gets canceled out by destructive interference.[117, 128] If this equilibrium is disturbed the cornea gets opaque (e.g. fibril swelling

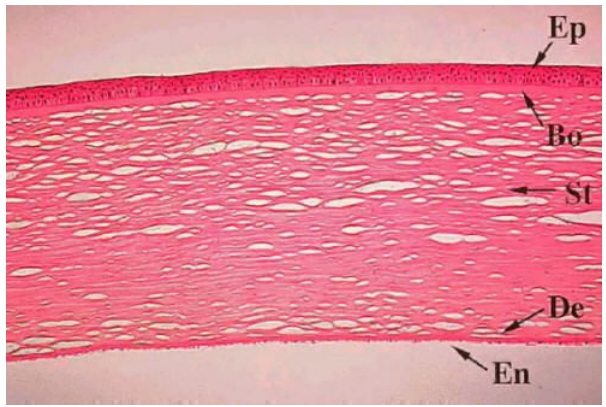

Figure 1.2: Histology of the cornea indicating the five layers: Ep=epithelium, $B o=$ Bowman layer, St=Stroma, $D e=$ Descemet membrane, En=edothelium. (Taken from www.flapandzap.com)

due to hydration). Being exposed to the environment on one side, the cornea as well as the sclera serve to protect the inner eye components. Thereby the combination of high rigidity and visco-elasticity allows absorbing physical injury (strokes etc.) and at the same time maintaining 
the ocular shape. The last one is especially important as the corneal refraction is very sensitive to small changes in corneal shape, smoothness and thickness which quickly can cause visual distortion. The corneal surface of an adult human is aspheric and convex. The cornea measures 11 to $12 \mathrm{~mm}$ in the horizontal diameter, 9 to $11 \mathrm{~mm}$ in the vertical diameter and its thickness shows a gradient increasing gradually from the center $520 \mu \mathrm{m}$ to the periphery $670 \mu \mathrm{m}$. Population average values for the radius of curvature is $7.8 \mathrm{~mm}$ for the anterior surface and $6.5 \mathrm{~mm}$ for the posterior surface. Corneal curvature is steepest in the center and flatter peripherally providing an asphericity of about $Q=-0.3$. Externally the cornea is covered by the tear-film that helps maintaining a smooth surface. Interiorly the cornea is in touch with the aqueous humor getting provided with nutrients. The cornea consists of three cellular layers and two membranes which are explained in more detail here:

\section{Layers of the Cornea}

EPITHELium The epithelium is the outermost layer and consists of nonkeratinized stratified squamous epithelial cells. It is made of about six cell layers and provides a constant thickness of about $50 \mu \mathrm{m}$. Epithelial cells proliferate from stem cells in the limbus and grow very fast. It takes a few days to recover after epithelial abrasion. Primarily the epithelium controls corneal hydration from the anterior side by preventing liquids to enter into the stroma. The epithelium also contributes to establish a smooth anterior surface. Irregularities lead to an alteration of the tear-film causing a significant alteration of corneal refraction. Only a negligible contribution to corneal stiffness is attributed to the epithelium. [54]

BOWMAN'S LAYER The Bowman's layer is an non-cellular layer of about 8-14 $\mu \mathrm{m}$ thickness and consists of condensed and irregularly arranged collagen type I (primarily) and III fibrils. Although the type of collagen fibrils is the same as in the stroma, the fiber diameter in the Bowman's 
layer is slightly smaller (20 to $30 \mu \mathrm{m}$ ). Due to its higher stiffness, the Bowman's layer protects the corneal stroma. It does not recover after rupture and is absent in most non-primates.

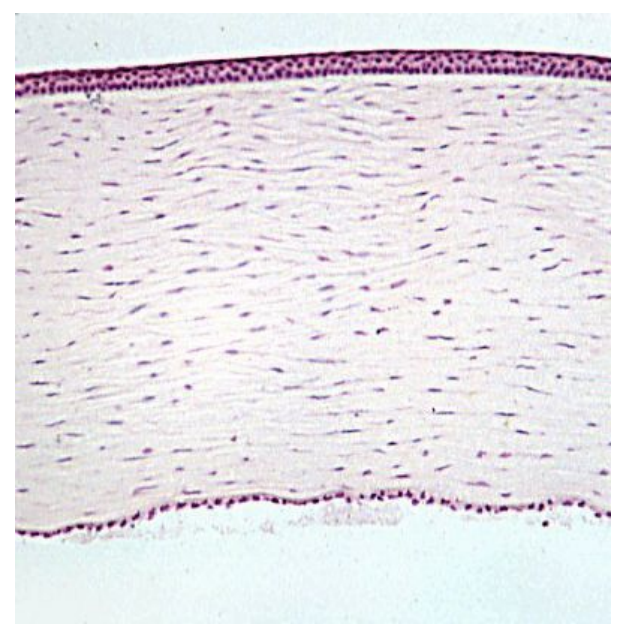

Figure 1.3: Histology of the cornea showing keratocyte nuclei. (Taken from simple-med.blgspot.)

STRomA The stroma makes out the largest part of the cornea (about 90\%) and therefore most biomechanical properties of the cornea are determined by this layer. The stroma is composed of an extracellular matrix, keratocytes (fibroblasts) and nerve fibers. About $70 \%$ of the dry weight consists of collagen molecules (mostly type I, but also type III, $\mathrm{V}$ and VI). Keratocytes synthesize pro- $\alpha$ chains. Three of them form a pro-collagen triple-helix, which then self-assembles into fibrils with an uniform diameter of 22.5 to $35 \mathrm{~nm}$. The highly regular organization of collagen fibrils with a homogenous inter-fibril distance (41.4 \pm 0.5 $\mathrm{nm}$ ) guarantees the high corneal transparency. Collagen fibers assemble into lamellae of about 1.5 to $2.5 \mu$ m thickness. The stroma contains about 200-300 lamellae layers going from limbus to limbus, parallel to the surface, but at various angles to one another. The space between collagen fibers is filled with glycosaminoglycans that together with core 
proteins form proteoglycans. Glycosaminoglycans are able to absorb large amounts of water and thus contribute to regulate the hydration state of the cornea. They provide a swelling pressure of $50 \mathrm{mmHg}$ which counteracts against the pump function of the endothelium. Keratocyte cells are distributed throughout the stroma and interconnected with each other. They take the role of general repair.

DUA'S LAYER Very recently this layer of about $10.15 \mu \mathrm{m}$ has been proposed by Dua et al [47]. It hypothetically consists of about 5 - 8 tightly packed predominantly type I collagen lamella with a fibril diameter of 24 $\mathrm{nm}$. The layer is supposed to have a high stiffness and hence could be important in corneal biomechanics.

Descemet Membrane The Descemet's membrane, increases its thickness from birth ( $3 \mu \mathrm{m})$ to adulthood (8-10 $\mu \mathrm{m})$. It consists of collagen type IV, laminin and fibronectin. Collagen type IV fibrils are about 5-20 $\mu \mathrm{m}$ thick and weaker than type I fibrils. The main purpose of the Descemet membrane is to hinder the aqueous humor from diffusing into the stroma. After rupture it does not regenerate. When stromal swelling occurs the Descemet membrane gets folded.

ENDothelium The endothelium is the last cellular mono-layer of the cornea and forms the border to the aqueous humor. Its cells are polygonal (with a diameter of $20 \mu \mathrm{m}$ and a thickness of $5 \mu \mathrm{m}$ ). The endothelium is the most important component of the cornea to regulate its hydration. There exists an osmotic gradient between the stroma (134 mEq/l) and the aqueous humor (143 mEqu/l), which results in a flux of sodium $\left(\mathrm{Na}^{+}\right)$from the aqueous humor and a flux of potassium $\left(\mathrm{K}^{+}\right)$from the stroma. Endothelial cells express $\mathrm{Na}^{+}$- and $\mathrm{K}^{+}$-dependent ATPase and $\mathrm{Na}^{+}$$\mathrm{K}^{+}$exchanger. Then, in combination with carbon dioxide bicarbonate ions $\left(\mathrm{HCO}_{3}^{-}\right)$are expressed and get pumped into the aqueous humor. Coupled to this $\mathrm{HCO}_{3}^{-}$movement is a flux of water out of the stroma. This transport mechanism depends on cellular activity and therefore declines 
with cooling, which results in increasing thickness and transparency loss. Up to a certain temperature thickness and clarity are reversible. Endothelial cell density decreases with age (from $3000-4000$ cells $/ \mathrm{mm}^{2}$ in newborns to 2000 cells $/ \mathrm{mm}^{2}$ at old age), whereby the cell diameter increases from $18-20 \mu \mathrm{m}$ to $40 \mu \mathrm{m}$ (polymegathism).

\section{Refractive Power}

The cornea is the main refractive interface of the eye with its power of 4044 diopters. Due to its convex spherical shape and the larger difference in refractive index at the anterior cornea $\left(n_{\text {air }}=1.0 \rightarrow n_{\text {cornea }}=1.375\right)$ than at the posterior cornea $\left(n_{\text {cornea }}=1.375 \rightarrow n_{\text {aqu.humor }}=1.336\right)$, the anterior corneal curvature contributes to about $90 \%$ of the corneal refractive power and to about $65 \%$ of the absolute refractive power of the eye. Because of this strong refractive contribution corneal irregularities and differences from the ideal aspherical shape are an important factor in visual degradation. For example, an elliptically shaped cornea leads to astigmatism. In order to provide an even corneal surface, the eye is covered by a constantly being refreshed tear-film. With aging often the tear-film production decreases, the cornea dries and its surface becomes more rough. The eye is a natural optical system and is composed of different optical components with different refractive indicies: tear fluid (1.336), corneal tissue (1.376), aqueous humor (1.336), vitreous humor (1.336), crystalline lens (gradient index, from 1.406 in the center to 1.386 in the outer layers).

\section{Aging}

Aging induces changes in the collagen structure of the human cornea, which is attributed to a three dimensional growth of the collagen fibrils [41]. Thereby the collagen fibril diameter, the axial period and the intermolecular Bragg spacing increase [41], which can be attributed to a progressive increase in the number of non-enzymatic (i.e. glycationinduced) cross-links over the years [127] that also stiffen [70] the corneal 
Chapter 1. Introduction

tissue. Corneas generally steepen in the horizontal meridian with age increasing the against-the-rule astigmatism among older patients [9].

\section{Optical background}

\section{Wavefront}

The wavefront describes a theoretical surface that connects all points in space that are reached by a propagating light wave at a given time. Practically, the propagation of a wavefront through an optical system gives information on its aberrations.

\section{Aberrations}

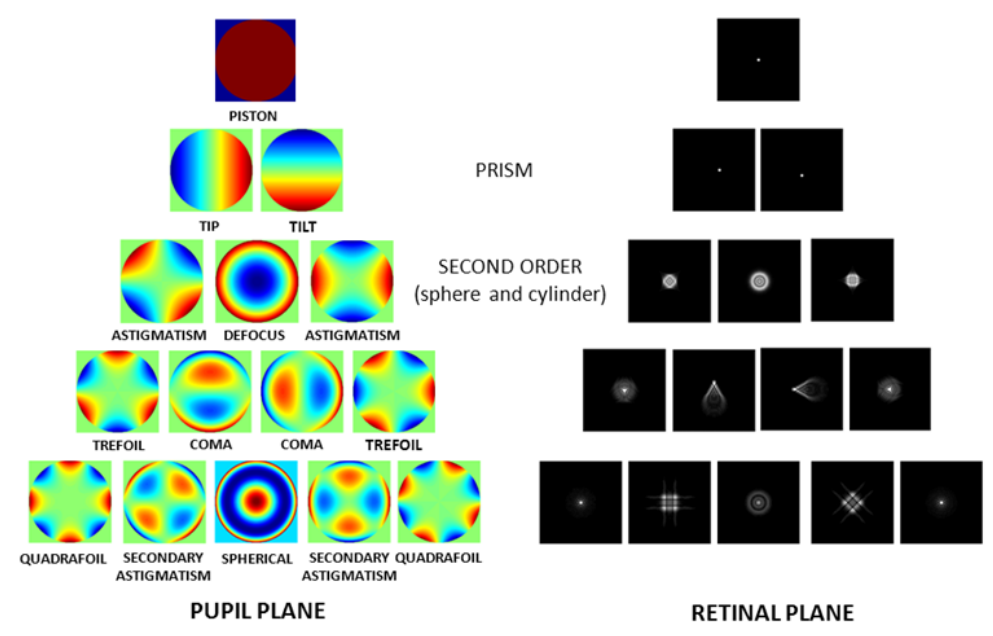

Figure 1.4: Left panel: basic shape of the first 15 Zernike polynomials; right panel: corresponding point-spread-functions, i.e. the way a punctual light source is imaged under the given aberration. Taken from [212].

Optical aberrations are apart from scatter and diffraction the main limitations of optical quality. Aberrations can be separated into monochro- 
matic wave aberrations and longitudinal, transverse chromatic aberrations, while the former are more important in visual science. Wave aberrations describe the deviation of a real wavefront present in an optical system, with respect to the ideal wavefront. According to Fritz Zernike [234] wave aberrations can be described by the sum of a set of orthogonal and hence mathematically (theoretically) independent polynomials, see Figure 1.4. Each of those polynomials is normalized to a unit circle and represents a specific part of the optical wave aberration. The Zernike polynomials $Z_{n}^{ \pm m}$ are defined by

$$
Z_{n}^{ \pm m}(\rho, \phi)=N_{n}^{m} \cdot R_{n}^{m}(\rho) \cdot G_{n}^{m}(\phi)
$$

and consist of a normalization factor $N_{n}^{m}$, a radial dependent component $R_{n}^{m}(\rho)$ and a sinusoidal azimuthal dependent component $G_{n}^{m}(\phi)$.

$$
R_{n}^{m}(\rho)=\sum_{k=0}^{(n-m) / 2} \frac{(-1)^{k}(n-k) !}{k !((n+m) / 2-k) !((n-m) / 2-k) !} \rho^{n-2 k}
$$

Here $\rho$ is the radial variable ranging from 0 to $1, \theta$ is the azimuthal variable ranging from 0 to $2 \pi, n$ is a positive integers representing the radial order and $m$ is an integer representing the azimuthal order. Table 1.1 summarizes the expanded equation 1.1 up to $5^{\text {th }}$ order. Typically aberrations are described by Zernike coefficients $z_{\text {num }}$ where num $=$ $\frac{n(n+1)}{2}+\frac{n+m}{2}$, which are factors that determine how much each polynomial contributes. The overall wavefront aberration can then be reconstructed using the following equation:

$$
W(\rho, \phi)=\sum_{n, m} z_{\text {num }} \cdot Z_{n}^{ \pm m}(\rho, \phi)
$$

\section{Refractive Errors of the Eye}

This section describes brevely the most common refractive errors in human eyes. 
Chapter 1. Introduction

Table 1.1: Zernike polynomials used to describe optical aberrations. $x$, stands for horizontal and $y$ for vertical.

\begin{tabular}{rlrl}
\hline$z 0$ & $=1 ;$ & & Piston or Bias \\
$z 1$ & $=\rho \cos (\theta) ;$ & & Tilt x \\
$z 2$ & $=\rho \sin (\theta) ;$ & & Tilt y \\
$z 3$ & $=-1+2 \rho^{2} ;$ & & Power or Defocus \\
$z 4$ & $=\rho^{2} \cos (2 \theta) ;$ & & Astigmatism x \\
$z 5$ & $=\rho^{2} \sin (2 \theta) ;$ & & Astigmatism y \\
$z 6$ & $=\rho\left(-2+3 \rho^{3}\right) \cos (\theta) ;$ & & Coma x \\
$z 7$ & $=\rho\left(-2+3 \rho^{3}\right) \sin (\theta) ;$ & & Coma y \\
$z 8$ & $=1-6 \rho^{2}+6 \rho^{4} ;$ & & Primary Spherical \\
$z 9$ & $=\rho^{3} \cos (3 \theta) ;$ & & Trefoil x \\
$z 10$ & $=\rho^{3} \sin (3 \theta) ;$ & & Trefoil y \\
$z 11$ & $=\rho^{2}\left(-3+4 \rho^{2}\right) \cos (2 \theta) ;$ & & Secondary Astigmatism x \\
$z 12$ & $=\rho^{2}\left(-3+4 \rho^{2}\right) \cos (2 \theta) ;$ & & Secondary Astigmatism y \\
$z 13$ & $=\rho\left(3-12 \rho^{2}+10 \rho^{4}\right) \cos (\theta) ;$ & & Secondary Coma x \\
$z 14$ & $=\rho\left(3-12 \rho^{2}+10 \rho^{4}\right) \sin (\theta) ;$ & & Secondary Coma y \\
$z 15$ & $=-1+12 \rho^{2}-30 \rho^{4}+20 \rho^{6} ;$ & & Secondary Spherical \\
$z 16$ & $=\rho^{4} \cos (4 \theta) ;$ & & Tetrafoil or Quadrafoil x \\
$z 17$ & $=\rho^{4} \sin (4 \theta) ;$ & & Tetrafoil or Quadrafoil y \\
$z 18$ & $=\rho^{3}\left(-4+5 \rho^{2}\right) \cos (3 \theta) ;$ & & Secondary Trefoil x \\
$z 19$ & $=\rho^{3}\left(-4+5 \rho^{2}\right) \sin (3 \theta) ;$ & & Secondary Trefoil y \\
\hline
\end{tabular}


MYopiA Myopia - also known as nearsightedness - is present when the focus of the eye falls in front of the retina causing a spherically defocused image. Myopia typically is corrected with a concave lens adding negative diopters.

HYPEROPIA Hyperopia - also known as farsightedness - is present when the focus of the eye falls behind the retina causing a spherically defocused image. Hyperopia typically is corrected with a convex lens adding positive Diopters.

Astigmatism Astigmatism is present when the ocular refraction is different in the horizontal and vertical axis. This means that if the focus of one meridian falls onto the retina, the focus of the other meridian falls before or behind resulting in an orientation dependent blur of the image (e.g. horizontal lines appear more blurred than vertical lines).

Coma Coma is present when the focus at a certain angle to the optical axis differs locally. This means that a point light source appears as a bright point with a less intense shade in one direction, similar to a comet (comet - coma: this is where the name comes from).

\section{Ray-tracing system}

Ray-tracing is a technique that tracks the way a light beam travels along its optical path. In ophthalmology this technique is used to measure the forward aberrations of the eye. Typically, narrow beams of light (from a point source) are projected sequentially and parallel to the line of sight throughout the pupil onto the retina and the position of the reflected retinal image (spot) is captured by a position sensitive detector or a CCD camera. Thereby each spot represents the ocular refraction at a given location of the pupil. If the eye is emmetropic, all spots will be observed on the fovea. In contrast if aberrations are present, the actually observed spot will be shifted out of the fovea. The wavefront of the eye 
can be reconstructed by analyzing the difference between the expected (fovea) and the actual location of the retinal image. Figure 1.5 depicts the working principle.

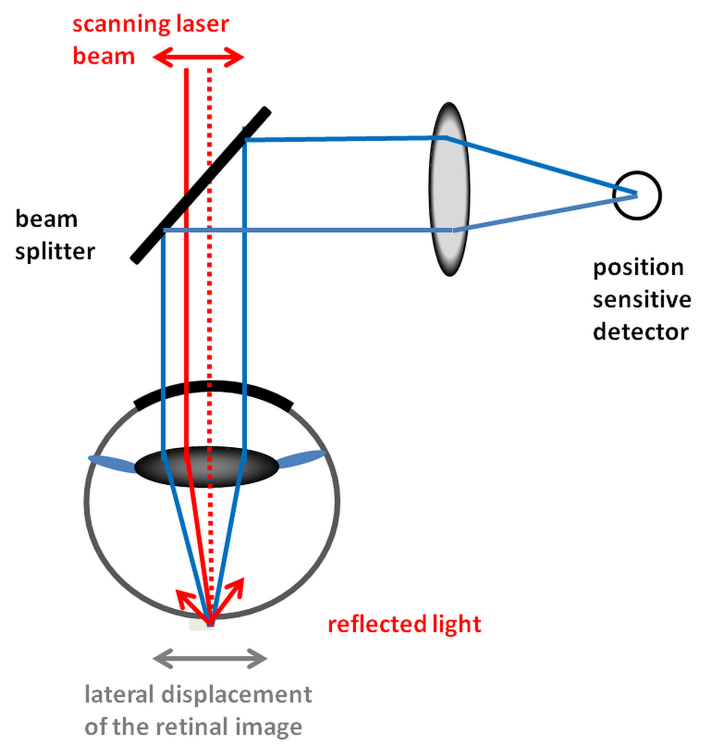

Figure 1.5: Principle of ray-tracing aberrometry.

\section{Coherence}

Coherence is an attribute of a propagating wave describing the limits wherein a constant relative phase exists. This means, if the given wave is divided in two parts, the phase of the second part can be predicted knowing the phase of the first part. Thereby temporal coherence refers to the correlation between the wave at different times at the same location. The coherence time then is defined as the time wherein no significant phase delay can be observed. Spatial coherence refers to the correlation between waves at different locations at the same time. The coherence length is defined as the distance the wave travels within the coherence time. 


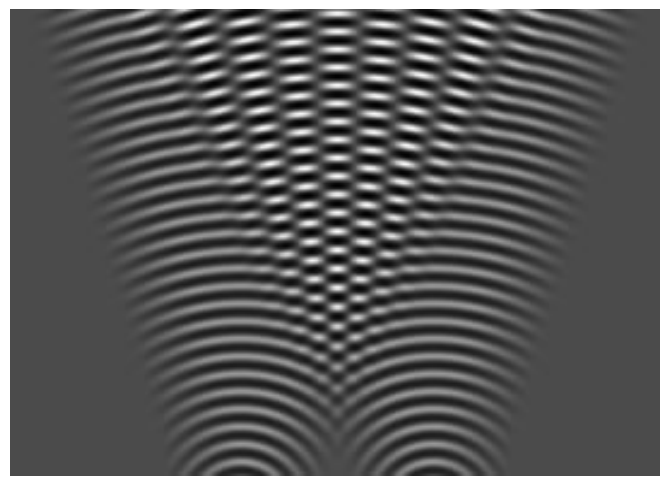

Figure 1.6: Interference pattern, taken from www.goldberg.lbl.gov.

\section{Interference}

Interference refers to the interaction of two coherent waves (typically light or other electromagnetic waves). The superposition of both signals results in an interference pattern depending on the phase differences: Inphase waves lead to constructive, out-of-phase waves lead to destructive interference. One of these simpler patterns are fringes, which are shown in Figure 1.6.

\section{Anterior segment imaging techniques}

\section{Scheimpflug Imaging}

Scheimpflug imaging is based on the Scheimpflug's principle, which goes back to Theodor Scheimpflug and describes a method to increase the depth of focus. In a typical optical system, the object and image plane are parallel to the lens plane. If this alignment is changed, so that the object plane is tilted respect to the lens plane, then the orientation of the plane of focus can be determined by geometric rules: The Scheimpflug's principle states that if the object plane is tilted, a sharp image can only be obtained when also the image plane is tilted. Figure 1.7 illustrates the Scheimpflug principle. Scheimpflug imaging allows the extension of 
Chapter 1. Introduction

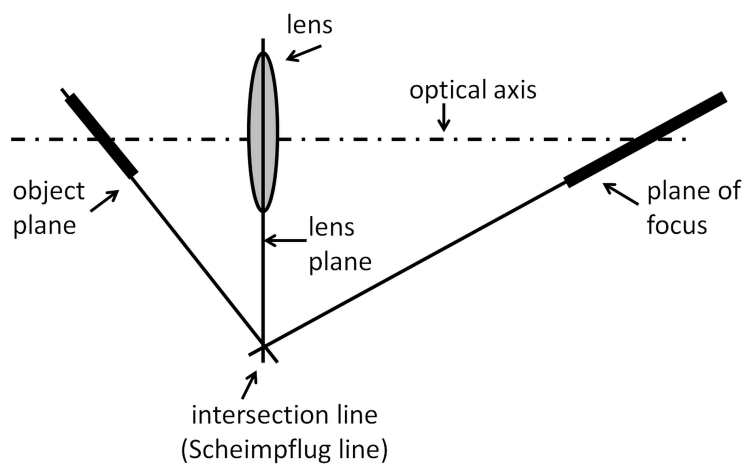

Figure 1.7: Scheimpflug's principle.

depth of focus, however the obtained images are affected by two kinds of distortion: Geometrical distortion occurs due to the tilt in the image
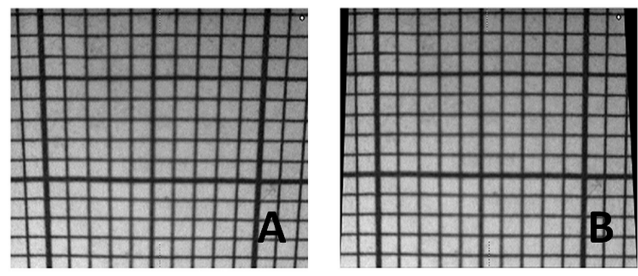

Figure 1.8: Geometrical distortion observed in Scheimpflug images.

plane, which makes the image appear trapezoidal distorted (see Figure 1.8) and optical distortion occurs as posterior optical surfaces are viewed through preceding refractive surfaces. Both distortions must be corrected in order to obtain quantitative information from Scheimpflug images. Figure 1.9 shows the effect of distortion correction.

\section{Optical Coherence Tomography}

Tomographic techniques create a set of sliced images (cross-sections) of 3D objects and are especially important in medicine as they allow a non-invasive insight into the human body. Optical coherence tomography 

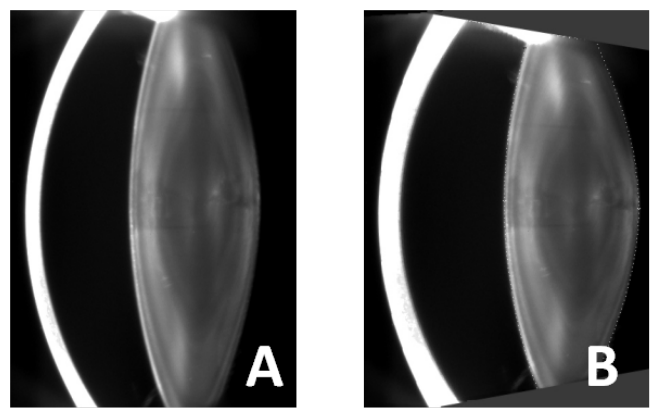

Figure 1.9: Scheimpflug image of the eye: (A) uncorrected, (B) corrected from distortions.

(OCT) allows a tomographic view of the tissue similar to ultrasound. Thereby the use of light allows a higher resolution and does not require contact as ultrasound waves, although the penetration in scattering tissue is generally lower. Ocular tissues are very suitable for the OCT imaging modality as they are highly transparent.

OCTs typically use a low time-coherent light source and scan the zone of interest in two directions: in depth and laterally. The depth scan either is performed by moving the reference mirror (time-domain OCTs) or by using a light source with wider bandwidth and splitting up the interference signal (spectral-domain OCTs). The lateral scan is generally performed by moving the object beam across the sample.

The working principle of OCTs is based on laser interference. First the coherent light is separated in two arms, one serves as reference beam, the other one is focused onto the sample. Then the reflected signal from the sample returns, joins the reference beam and both interfere with each other. There exist different types of OCTs. Here the spectral OCT and the swept-source OCT are presented as they were used for the experiments presented in this thesis. In spectral OCT a broadband light source is used and the spectral components of the interference signal are captured simultaneously and after separated by a spectrometer; in swept-source OCT a narrow band laser source is used and the spectral components 
of the interference signal are captured sequentially while sweeping the laser-wavelength [97].

\section{Ultrasonic Imaging}

Conventional sonography is based on a similar interference theory as optical coherence tomography, only that the resolution of the images is much lower (see Figure 1.10), while the penetration depth is larger. Applied to the eye, sonography can be used to image the anterior segment

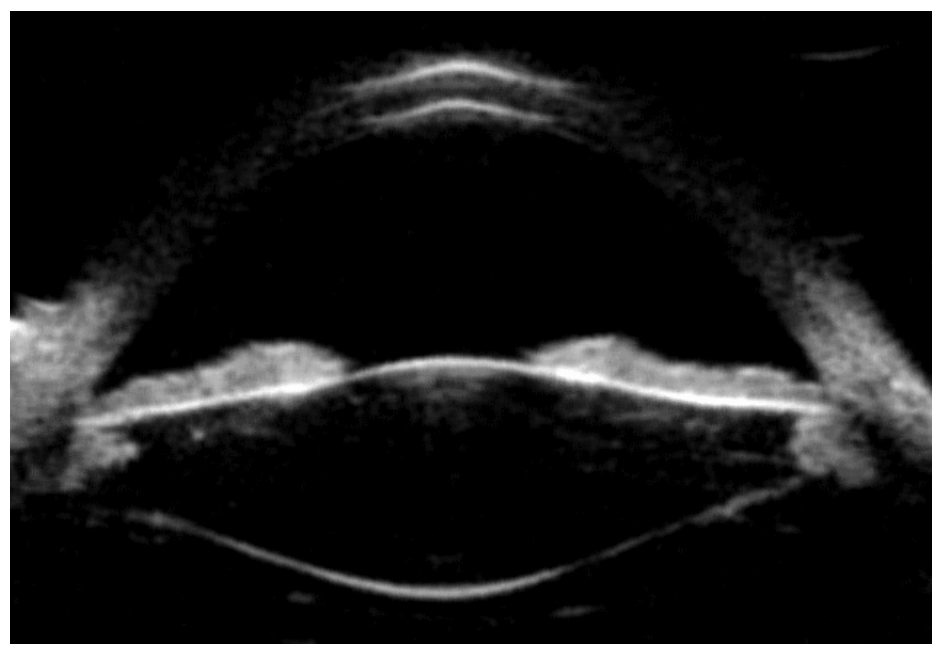

Figure 1.10: Typical image obtained from an ultrasonic biomicroscope. Here the focus is adjusted to the lens plane. The cornea appears deformed as optical distortion has not been corrected. The resolution is much lower compared to OCT images.

of the eye, reaching from the cornea back to the lens. A disadvantage of ultrasound imaging is its relative low resolution. Recently, with the purpose of estimating tissue mechanical properties, a new ultrasound imaging-based "shear wave elasticity imaging" has been suggested [173]. The technique has the potential to assess quantitatively the elastic and viscoelastic parameters of tissue, as shear waves can induce mechanical oscillations in a locally very limited zone. The elastography technique is 
based on the relationship of the shear wave propagation speed $c=\sqrt{\frac{\mu}{\rho}}$ and the biomechanical properties of the tissue:

$$
\frac{\delta^{2} s_{x}}{\delta t^{2}}-\left(c^{2}+v \frac{\delta}{\delta t}\right) \Delta s_{x}=F_{x}
$$

where $\mu$ is the shear elastic modulus, $v=\frac{\eta}{\rho}$ is the kinematic shear viscosity, $s_{x}$ is the displacement component along the beam direction and $F_{x}$ is the radiation force in beam direction. Ultrasonic elastography has allowed to estimate the corneal Young's modulus to be about $190 \mathrm{kPa}$, while corneal viscosity typically was neglected as it would require a higher imaging speed than that compatible with current commercial ultrasound scanners. New versions of ultrasound elastography and its analysis are still being developed [32].

\section{Mircroscopic imaging of the cornea}

\section{X-ray scattering}

X-ray scattering has been applied to study quantitatively the orientation and distribution of the collagen molecules, fibrils and lamellae within the corneal tissue $[130,136]$. Generally, when a focused x-ray beam passes through an ordered fibrous structure, the scattered waves interfere and result in a x-ray scatter pattern, which contains micro- and nanoscopic information on the structural organization of the sample, such as shown in Figure 1.11. Typically two operation modes are used: recording small-angle scattering, which is caused by collagen on the fibril level; or recording wide-angle scattering, which is caused by the collagen molecules within the fibrils. Most important findings from x-ray scattering include that the collagen fibril orientation differs between species. Humans have a predominant orthogonal fibril orientation at the central cornea, while pigs, rabbits and mouses have a circumferential orientation. Thereby orthogonal orientation was related to a higher visual acuity than vertical or circumferential orientation [84]. In human keratoconic 
Chapter 1. Introduction
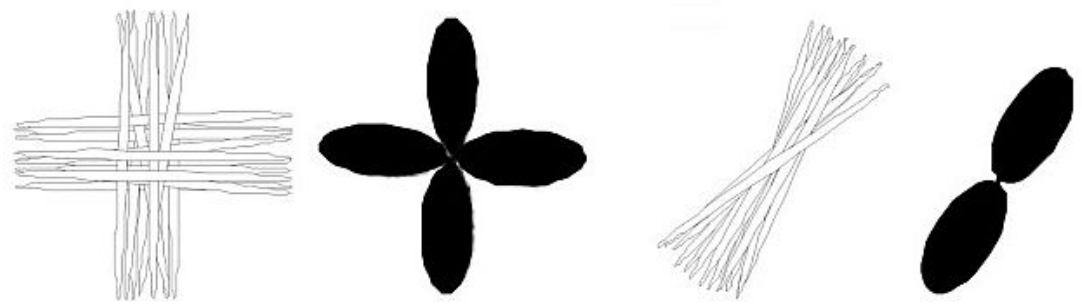

Figure 1.11: Differences in corneal orientation and the corresponding $x$-ray signal. Adapted from [136].

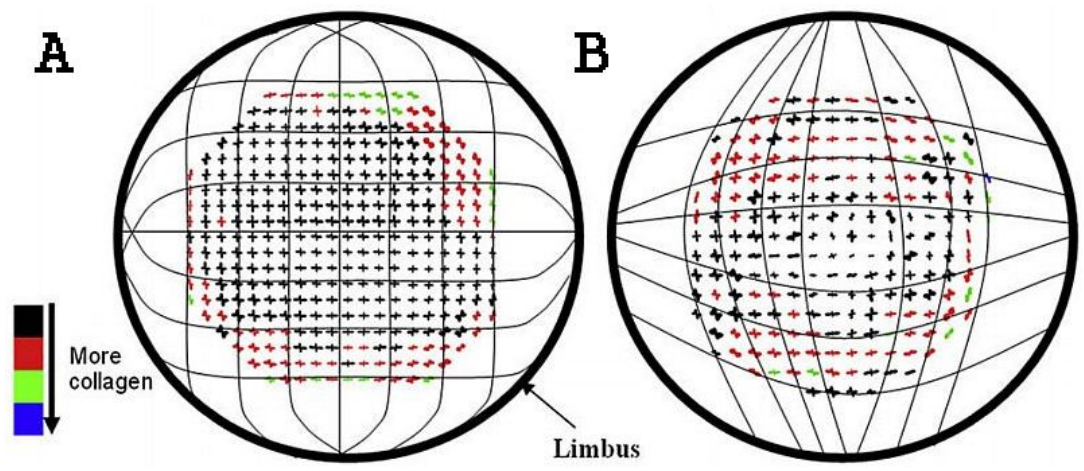

Figure 1.12: Differences in collagen orientation in a normal and keratoconic cornea. Adapted from [130]. 
corneas the orthogonal fibril orientation was disturbed (see Figure 1.12).

\section{Second harmonic generation microscopy}

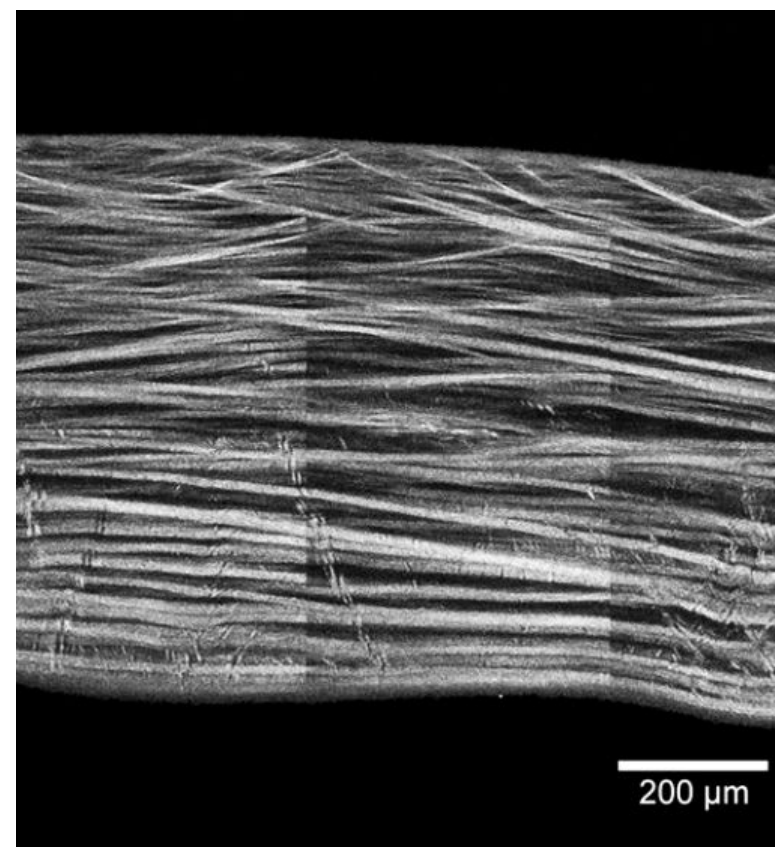

Figure 1.13: Second harmonic generated images showing a higher collagen fiber branching in the anterior cornea compared to the posterior cornea. Adapted from [219].

Second harmonic imaging is based on the principles of multiphoton microscopy and requires intense laser light - typically femtosecond pulses from a mode-locked titanium-sapphire laser of $800 \mathrm{~nm}$ wavelength - and a non-centrosymmetric molecular structure, such as collagen, to generate the second-harmonic signal. Second harmonic generation is a non-linear optical effect, in which the incident photons interact with the specimen creating new photons with twice the energy. This results in a forwardand backscattered frequency doubled second harmonic light containing information on the fibril structure of the cornea. Most important findings 
from corneal second harmonic imaging are the presence of a higher interweaving and branching of collagen fibrils in the anterior compared to the posterior cornea (see Figure 1.13). Thereby a four times higher branching density was observed in the anterior third of the cornea, which was confirmed by indentation tests revealing a difference by factor eight between the anterior and posterior stroma [219]. Furthermore sutural fibers inserting into Bowman's layer were observed and differences in the collagen lamellae distribution after cross-linking, suggesting the creation of abnormal structures compared to the regular distribution in virgin corneas [23]. Inter-specie differences have been reported [22] regarding the collagen lamella interweaving, their length and the fibril orientation.

\section{Brillouin microscopy}

Brillouin light scattering arises from the interaction between photons and acoustic phonons (propagation of thermodynamic fluctuations) in a sample. Thereby the Brillouin scattering happens at a wavelength between Rayleigh and Raman scattering. The elastic Rayleigh scattering is caused by particles much smaller than the incident wavelength and does not produce any frequency shift. In contrary, the inelastic Raman scattering is caused by interference of the incident wavelength with the molecular energy (electronic or vibrational excitation) and is typically used in chemistry for material analysis. In the inelastic Brillouin scattering, the frequency shift $\Omega$ and the linewidth $\Delta \Omega$ of the Brillouin spectrum are related to the longitudinal viscoelastic modulus $M^{*}$ of the sample, which is composed of elastic modulus, M', and viscous modulus, M"

$$
M^{\prime}=\frac{\rho \lambda^{2} \Omega^{2}}{4 n^{2}} M^{\prime \prime}=\frac{\rho \lambda^{2} \Omega \triangle \Omega^{2}}{4 n^{2}}
$$

where $\lambda$ is the mass density, $\mathrm{n}$ is the optical wavelength and $\mathrm{n}$ is the refractive index. To convert the Brillouin shift to the longitudinal modulus, the value of $\rho / n^{2}$ is required. From literature values of the refractive index and density, which are spatially varying, $[105,217]$, the ratio of 
$\rho / \mathrm{n}^{2}$ is found to be approximately constant with a value of $0.57 \mathrm{~g} / \mathrm{cm}^{3}$ and a variation of less than $0.3 \%$ throughout the cornea[85, 146].

Using Brillouin scattering to assess the elastic properties of corneal tissues was proposed by Vaughan et al [211] for the first time. However the acquisition was slow and only individual points could be measured at a time. Scarcelli et al [178] improved the one-point-samping procedure by using a parallel spectrometer, which increased dramatically the speed of measurements (nearly 100-fold) and allowed an imaging modality. The successful imaging of the stiffness gradient along the cornea has been demonstrated in vitro [177], but also first measurements have been performed in the in vivo eye [180].

\section{Corneal disease and treatment background}

\section{Corneal Pathologies}

\section{Keratoconus}

Keratoconus is a pathology affecting primarily young patients. Keratoconus causes structural changes in the collagen organization of the stroma [132, 137] - which probably weakens the corneal tissue [6] - and leads to corneal thinning [116] and a conical out-bulging. This outbulging progresses and causes increased visual degradation. Due to gravity the cone is typically located slightly below the corneal center. In most cases it is furthermore displaced slightly towards the temporal direction. This leads to the typical coma aberration observed in patients. Keratoconus often affects both eyes, yet differences in the severity between both eyes are common.

Physical eye examinations, which allow the diagnosis of keratoconus, include: visual acuity (Snellen chart), refraction (Keratometer) and slit lamp examination. The severity of keratoconus is classify by the Amsler-Krumeich scale (see Table 1.2).

For moderate keratocones optical distortions are reduced by contact 
Chapter 1. Introduction

Table 1.2: Amsler-Krumeich scale to classify keratoconus severity.

\begin{tabular}{|c|c|}
\hline Stage & Clinical observations \\
\hline \multirow[t]{2}{*}{$\mathrm{I}$} & Corneal radius $\leqslant 48 \mathrm{D}$ \\
\hline & Myopia / astigmatism < 5D \\
\hline \multirow[t]{3}{*}{ II } & Corneal radius $\leqslant 53 \mathrm{D}$ \\
\hline & Myopia / astigmatism $>5 \mathrm{D}<8 \mathrm{D}$ \\
\hline & Pachymetry $\leqslant 400 \mu \mathrm{m}$ \\
\hline \multirow[t]{3}{*}{ III } & Corneal radius $>53 \mathrm{D}$ \\
\hline & Myopia / astigmatism $>8 \mathrm{D}<10 \mathrm{D}$ \\
\hline & Pachymetry $200-400 \mu \mathrm{m}$ \\
\hline \multirow[t]{2}{*}{ IV } & Corneal radius $>55 \mathrm{D}$ \\
\hline & Pachymetry $<200 \mu \mathrm{m}$ \\
\hline
\end{tabular}

lenses acting as a mold to reshape the cornea. However further advanced keratocones require surgery. Some surgical procedures address the correction of the corneal shape (such as intrastromal ring segments); others address the progression of the out-bulging cone (such as UV corneal collagen cross-linking); or the replacement of the diseased cornea by donor tissue (such as keratoplasty).

\section{Corneal Treatments}

Several corneal treatments rely in the corneal biomechanical response to produce changes in corneal shape. These include early incisional refractive surgery (i.e. radial keratoctomy), or techniques that involve the implantation of corneal implants or inlays to reshape the cornea. Other treatments aim at increasing the corneal stiffness in order to halt corneal ectasia. This section explains the working principle of the surgeries and treatments studied in this thesis.

\section{UV Corneal Collagen Cross-linking}

UV-Corneal Collagen Cross-linking (CXL) was proposed in 1998 by Spoerl et al.[195] as a procedure to increase corneal stiffness and therefore halt the progression of keratoconus. The biomechanical properties of collagen 
tissues depend largely on their number of cross-links. In general the more cross-links, the stiffer the tissue. CXL was designed to increase the number of cross-links thereby limiting the stiffening effect to the central cornea (typical irradiation zone of $10 \mathrm{~mm}$ ). Standard CXL takes an hour and generally requires epithelium removal to allow the photosensitizer (riboflavin) to diffuse into the stroma. After instilling riboflavin-dextran solution for 30 minutes the cornea is completely soaked with riboflavin. UVA irradiation is applied during 30 minutes. A new version of the treatment is currently under research that will not require the removal of the epithelium. The CXL treatment is based on a photo-dynamic principle [11]: Riboflavin has several absorption maxima in the UV range (270, $366,445 \mathrm{~nm}$ ). A $366 \mathrm{~nm}$ wavelength is applied for treatment, although it is not the maximal absorption peak. The reason is that the wavelength, where absorption is highest $(270 \mathrm{~nm})$, is mostly absorbed by the DNA, which would lead to severe damages of the genetic material. On the other hand the $445 \mathrm{~nm}$ wavelength is potentially hazardous to the retina. Riboflavin absorbs the incident light according to the Beer Lambert law:

$$
I=I_{0} \cdot 10^{-\varepsilon \cdot d \cdot c}
$$

where $I_{0}$ is the intensity of the incident light, $I$ is the transmitted intensity after passing a certain path $d$ in a liquid with the molar concentration $c$ and molar absorptivity $\varepsilon$ (material constant). The transmitted light excites the riboflavin molecules to its singlet and triplet state. Thereby the excited triplet state lives longer than singlet state and is more probable to react with the substrate.

riboflavin excitation

$$
\begin{aligned}
R f & \rightarrow R f_{1}^{*}, I_{a b s} \\
R f_{1}^{*} & \rightarrow R f, k 1 \\
R f_{1}^{*} & \rightarrow R f_{3}^{*}, k 2
\end{aligned}
$$


According to a recent study [102] the oxygen concentration determines the kinetics of the photochemical reaction and the type of the continuing onset reactions: Type I mechanism is dominant at low oxygen concentrations. The excited sensitizer (riboflavin) reacts with the substrate (cornea) forming radicals or radical ions by hydrogen atom or electron transfer:

$$
\begin{gathered}
\text { Type I mechanism } \\
R f_{3}^{*}+S H \rightarrow\left(R f^{-}+S H^{+}\right) \rightarrow R f H^{\prime}+S^{\prime}, k 3 \\
2 R f H^{\prime} \rightarrow R f+R f H_{2}, k 4 \\
R f H_{2}+O_{2} \rightarrow R f_{o x}+H_{2} O_{2}, k 5
\end{gathered}
$$

Type II reaction is dominant at higher oxygen concentrations. Here the excited sensitizer reacts with the oxygen creating singlet molecular oxygen:

Type II mechanism

$$
\begin{gathered}
\left.R f_{3}^{*}+\right)_{2} \rightarrow R f+{ }^{1} \mathrm{O}_{2}, k 6 \\
S H+{ }^{1} \mathrm{O}_{2}^{\rightarrow S_{o x}}, k 7
\end{gathered}
$$

Singlet oxygen, also referred to as free oxygen radical, is extremely reactive and attributed to form new covalent bonds between and/or within collagen fibrils. However a direct proof of new cross-links after CXL has not been achieved yet.

In the previous equations $R f, R f_{1}^{*}$ and $R f_{3}^{*}$ stands for riboflavin in its ground state, singlet excited state and triplet excited state, respectively. $R f^{-}$is the radical anion, $R f H^{\prime}$ the radical and $R f H_{2}$ the reduced form of riboflavin. $\mathrm{SH}$ is the substrate, $S H^{+}$is the intermediate radical cation, $S^{\prime}$ the radical and $S_{o x}$ the oxidized form or the substrate. $R f_{o x}$ is the oxidized form of riboflavin with a similar UV-A absorption as riboflavin. 


\section{Intrastromal Corneal Ring Segments}

ICRSs have been proposed as a therapeutic tool to increase the biomechanical stability of keratoconic corneas and to improve their regularity. ICRS are also used to correct high astigmatism and myopia. ICRSs are made of PMMA, have typically a triangular, hexagonal or oval crosssection and are available in several dimensions (typically, $4.40 / 5.60 \mathrm{~mm}$ to $6.77 / 8.10 \mathrm{~mm}$ inner/outer radii of curvature and from $90^{\circ}$ to $210^{\circ}$, $150-350 \mu \mathrm{m}$ height and 600-800 $\mu \mathrm{m}$ width). For implantation a tunnel parallel to the cornea is created at 70\% depth of the tissue [118] in which the ICRS is inserted after. Clinical studies report on curvature changes ranging form -1 to -3.5 diopters[189] after ICRS implantation, as well as a small rotation $[36,147]$ of the ICRS.

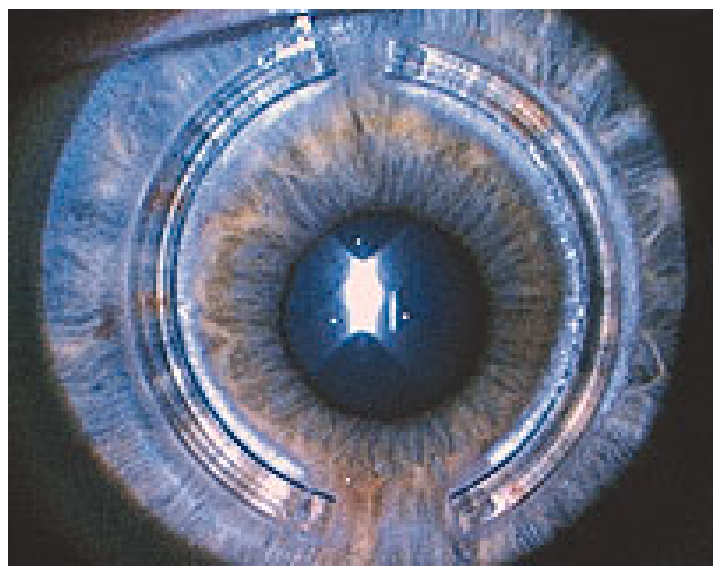

Figure 1.14: Intrastromal ring segments implanted in a human cornea. (Taken from [63]). 


\section{Mechanical background}

\section{Finite Element Method}

In 1909 Ritz [167] developed a technique to approximate the deformation of solids and set herewith the beginning of the finite element method. His technique was based on the minimization of energy estimations in order to determine unknown coefficients. The method was further improved in 1943 [39] when hat functions were introduced as new coordinate functions removing restrictions regarding the boundary conditions. Nevertheless the approach did not spread out, due to the high computational demand, until 1960 when Clough [35] suggested independently a very similar technique and introduced the term "finite element method" for the first time.

The finite element method is based on the equilibrium state, i.e. on a system relying on physical and geometrical equations. Typically the potential energy $\Pi$ is defined in variational formulation:

$$
\Pi(u(x))=U-W=\frac{1}{2} \int_{0}^{l} E F\left(\frac{d u}{d x}\right)^{2} d x-\int_{0}^{l} q u d x
$$

where $U$ is the strain energy stored in the system, $W$ is the lost energy through external forces $F$. $E$ is the modulus of elasticity, $u$ the displacement, $d u$ the increment and $q$ a constant (assuming linear material properties). This equation is then discretized into finite elements and expressed as a function of nodal displacements:

$$
\Pi^{e}=\frac{1}{2}\left\{u_{1} u_{2}\right\}\left(\begin{array}{ll}
K_{11} & K_{12} \\
K_{21} & K_{22}
\end{array}\right)\left\{\begin{array}{l}
u_{1} \\
u_{2}
\end{array}\right\}-\left\{u_{1} u_{2}\right\}\left\{\begin{array}{l}
F_{1} \\
F_{2}
\end{array}\right\}=\frac{1}{2} d^{e T} K^{e} d^{e}-d^{e T} F^{e}
$$

where $K$ is the stiffness matrix, $d$ is the nodal displacement vector and $F$ the external nodal force vector. The system of algebraic equations is then solved (analytically or numerically) satisfying $\frac{\delta \Pi}{\delta u_{i}}=0$ and the 
boundary conditions.

\section{FEM Analysis in Ansys}

The complexity of equation 1.19 may be increased when including more material properties. Ansys software (ANSYS, Inc., Canonsburg, PA, USA) uses the following equation to generally describe motion in a structural system:

$$
[M]+[C]+[K] u=F^{a}
$$

where $[M]$ is the structural mass matrix, $[C]$ is the structural damping matrix, $[K]$ is the structural stiffness matrix, is the nodal acceleration vector, is the nodal velocity vector, $u$ the nodal displacement vector and $F^{a}$ the applied load vector. Imposed by the external stimulation, all nodes in the structure move at the same frequency, whereby the presence of damping or viscoelasticity induces phase shifts (making the natural frequencies and shapes complex). Forces and displacements can be expressed by complex notation:

$$
\begin{gathered}
u=u_{\max } e^{i \Phi} e^{i \Omega t}=\left(u_{1}+i u_{2}\right) e^{i \Omega t} \\
F=F_{\text {max }} e^{i \Psi} e^{i \Omega t}=\left(F_{1}+i F_{2}\right) e^{i \Omega t}
\end{gathered}
$$

Where $u_{\max }$ is the maximum displacement, $F_{\max }$ is the force amplitude, $i$ is the square root of $-1, \Omega$ is the imposed circular frequency expressed in radians/time $(2 \pi f), f$ is the impose frequency expressed in cycles/time, $t$ is time, $\Phi$ the displacement phase shift and $\Psi$ is the force phase shift. $u_{1}=u_{\text {max }} \cos \Phi$ and $\left\{F_{1}\right\}=\left\{F_{\text {max }} \cos \Psi\right\}$ are the real displacement and force vector, respectively; $u_{s}=u_{\max } \sin \Phi$ and $F_{1}=F_{\max } \sin \Psi$ are the imaginary displacement and force vector, respectively. Substituting equation 1.21 and 1.22 into 1.20 leads to the final equation of motion:

$$
\left([K]-\Omega^{2}[M]+i \Omega[C]\right)\left(\left\{u_{1}\right\}+i\left\{u_{2}\right\}\right)=F_{1}+i F_{2}
$$


Considering inertia, damping and static loads is important in a dynamic analysis. Inertia is proportional to the square of the imposed frequency $\Omega$ and depends on the element mass matrix $\left[M_{e}\right]$ :

$$
F_{1 e}^{m}+F_{2 e}^{m}=\Omega^{2}\left[M_{e}\right] u_{1 e}+\left[M_{e}\right] u_{2 e}
$$

The subscript $e$ refers to element. Damping is directly proportional to the imposed frequency and depends on the element damping matrix $\left[C_{e}\right]$ :

$$
F_{1 e}^{m}+F_{2 e}^{m}=-\Omega[C] u_{2 e}+\Omega\left[C_{e}\right] u_{1 e}
$$

Static loads depend on the element stiffness matrix $\left[K_{e}\right]$ are independent of the imposed frequency:

$$
F_{1 e}^{m}+F_{2 e}^{m}=-\left[K_{e}\right] u_{23}+\left[K_{e}\right] u_{1 e}
$$

In this context equation 1.23 can be expressed by

$$
\left[K_{c}\right] u_{c}=F_{c}
$$

The subscript $c$ stands for complex notation.

\section{Material Models}

Several models have been proposed to describe differences in the stressstrain relation for different kinds of materials. The simplest model description is linear elasticity, which is present in almost every material, however only withing a very small strain range. In order to describe materials at higher stresses, non-linear elastic and hyperelastic theories have been introduced. For isotropic materials it is sufficient to express stress and strain in only one direction. If there are reinforcements along one direction (such as fibers in wood, or collagen in the cornea), orthotropic (for orthogonal fiber orientation) or anisotropic (for any fiber orientation) properties need to be considered. All above mentioned ma- 
terial models refer to the inhered material structure and its organization. A different kind of non-linearity is viscoelasticity, which refers to time dependent non-linearity instead of strain dependent non-linearity. Table 1.3 summarizes different aspects of the most important material models for corneal tissue.

Table 1.3: Comparison of different material models.

\begin{tabular}{c|c|c} 
material model & dependency & deformation \\
\hline linear elasticity & none & reversible \\
non-linear elasticity & strain & reversible \\
viscoelasticity & time & reversible \\
hyperelasticity & strain & reversible \\
plasticity & strain, time & permanent \\
\hline
\end{tabular}

\section{Elasticity}

LINEAR ELASITICITY The behavior of linear elastic materials is described by the Hooke's law: $\sigma=k \cdot \epsilon$, with $\sigma=\frac{F}{A}$ and $\epsilon=\frac{L-L_{0}}{L_{0}}$, where $\sigma$ is stress, $\epsilon$ is strain and $k$ the elastic proportionality factor. It is valid for isotropic materials, i.e. where the material properties are independent on its orientation, the stress-strain relation over time is constant and deformations totally reversible. Only ideal materials show this behavior in all conditions. Nevertheless, the theory of linear elasticity can be applied in cases when e.g. stresses and strains are infinitesimally small. Also, many materials show an initially linear stress-strain relation up to the point where a certain yield stress is reached (see Figure 1.15). And, linear elasticity becomes valid in an isotropic viscoelastic material, when only very short time slots are analyzed, where the viscous elements do not have any impact.

NONLINEAR ELASTICITY Nonlinear elasticity refers to deformations that are not completely reversible (independent of time), i.e. the final deformation consists of both, an elastic part (reversible) and a plastic 
Chapter 1. Introduction

part (permanent).

\section{Viscoelasticity}

Viscoelastic theory is applied, when the stress-strain relation is time dependent (dynamic). Linear viscoelastic deformation consists of two parts, an elastic deformation (immediately reversible) and a viscous defromation (reversible, but time dependent), whereas nonlinear viscoelastic deformation additionally consists of a third plastic deformation (permanent). Most corneal models developed in this thesis consider linear viscoelasticity.

LiNEAR VISCOELASTICITY There exist several common formulations to describe the linear viscoelastic behavior. All of these combine two basic elements: A spring representing the linear elastic behavior $\sigma=k \cdot \epsilon$ and a dash-pot representing the linear viscous behavior $\sigma=v \cdot \frac{\delta \epsilon}{\delta t}$. When applying a step load, the spring element answers with an immediate constant increase in strain, while the dash-pot answers with creep (i.e. gradually increasing strain, until the maximum is reached). If this load is then released, the spring element answers with an immediate constant reduction of strain, while the dash-pot answers with relaxation (i.e. gradually decreasing strain, until the initial geometry is reached). In the Maxwell model, spring and dash-pot are in series and the constitutive equation results in: $\sigma+\frac{v}{k} \cdot \frac{\delta \epsilon}{\delta t}$ This model describes well the relaxation of a material, however fails for creep. In the Kevin-Voigt model spring and dash-pot are parallel, which results in: $\sigma=k \cdot \epsilon+\nu \cdot \frac{\delta \epsilon}{\delta t}$ This model in contrast describes well the creep of a material, but fails for relaxation. In order to account for both - creep and relaxation - the standard linearized model has been introduced. It consists of a spring which is parallel to a variable number of Maxwell elements. $\sigma(t)=\epsilon_{0} \cdot Y(t)+\int_{0}^{t} Y(t-\xi) \cdot \frac{\mathrm{d} \epsilon(\xi)}{\mathrm{d} \xi} \mathrm{d} \xi$ $\sum_{i=1}^{N} \alpha_{i} \cdot e^{\frac{-t}{\tau_{i}}}$

The stress in viscoelastic materials depends on the elastic and viscous 
properties, which can be described with the standard linear model:

$$
\sigma(t)=E \cdot \varepsilon+\eta \cdot \frac{\delta \varepsilon}{\delta t}
$$

where $\mathrm{E}$ is the elasticity modulus, $\varepsilon$ is the strain and $\eta$ the viscosity. The spring describes the instantaneous elastic contribution $(E \cdot \varepsilon)$ and the dash-pot the time dependent viscous contribution $\left(E \cdot \eta \cdot \frac{\delta \varepsilon}{\delta t}\right)$. Prony series were applied to describe the viscoelastic behavior. For this purpose the time dependent Cauchy stress $\sigma$ was expressed as

$$
\sigma=\int_{0}^{t} 2 G(t-\xi) \frac{d e}{d \xi} d \xi
$$

where e is the deviatoric part of the strain, $t$ is the current time and $\xi$ is the past time. The shear relaxation function $\mathrm{G}(\mathrm{t})$ is defined as

$$
\sigma=G_{0}\left[\alpha_{i}^{G} \exp \left(-\frac{t}{\tau_{i}^{G}}\right)\right.
$$

with

$$
G_{0}=G_{\infty}+\sum_{i=1}^{n_{C}} G_{i}
$$

and

$$
\alpha_{i}^{G}=\frac{G_{i}}{G_{0}}
$$

where $\alpha_{i}^{G}$ is the relative modulus, $G_{0}, G_{\infty}$ and $G_{i}$ are the instantaneous, the infinite and the current shear elastic moduli, respectively, and $\tau_{i}^{G}$ the relaxation times for each of the $n_{G}$ Prony components. In accordance with 1.32, the sum of relative moduli must be equal to 1 . In order to reduce the amount of material properties to be optimized, we limited the amount of Prony constants and set $n_{G}=1$. Thermal effects were not considered for the viscoelastic behavior. 
Chapter 1. Introduction

\section{Hyperelasticity}

In hyperelastic materials the strain is non-linear but reversible. The material is characterized by its strain energy density potential:

$$
S_{i j}=\frac{\delta W}{\delta E_{i j}}=2 \frac{\delta W}{\delta C_{i j}}
$$

Here $S_{i j}$ are components of the second Piola-Kirchhoff 260 stress tensor, $\mathrm{W}$ is the strain energy function per unit undeformed volume, $E_{i j}$ are components of the Lagrangian strain tensor and $C_{i j}$ are components of the right Cauchy-Green deformation tensor and can be expressed as $C_{i j}=F_{k i} F_{k j}$ where $F_{i j}$ are components of the deformation gradient tensor. The 3-parameter Mooney-Rivlin model can be used to express the strain energy as a scalar function of the deformation tensors:

$$
W=c_{10}\left(I_{1}-3\right)+c_{01}\left(I_{2}-3\right)+c_{11}\left(I_{1}-3\right)\left(I_{2}-3\right)+\frac{1}{d}(J-1)^{2}
$$

where $c_{10}, c_{01}, c_{11}$ and $\mathrm{d}$ are material constants, $I_{1}$ and $I_{2}$ are invariants of $C_{i j}, J=\operatorname{det}\left|F_{i j}\right|$ and $\mathrm{J}$ is the ratio of the deformed elastic volume over the undeformed volume of materials. For the simulations presented here, hyperelastic (anisotropic) behavior was necessary for higher strains; for small deformations the cornea still behaves isotropically. $d$ is the incompressibility parameter and was estimated by

$$
d=\frac{2}{K}=\frac{2 \cdot 3(1-2 v)}{E}
$$

where $\mathrm{K}$ is the bulk modulus, $\mathrm{E}$ the elasticity modulus and $v$ the Poisson's ratio. Due to its high water content the cornea can be considered incompressible, hence we defined $v=0.5$. The parameters $c_{10}, c_{01}$ and $c_{11}$ do not have a direct physical meaning, but for consistency with linear elasticity the shear modulus $\mu$ is given by

$$
\mu=2\left(c_{01}+c_{10}\right)
$$


for small strains. The shear modulus describes the material stiffness, i.e. the resistance when the top surface is displaced laterally respect to the bottom surface.

\section{Orthotropic Properties}

The structural composition of a material may cause a different behavior in different directions. This behavior is typically observed in fiber-reinforced materials. When the reinforced fibers are oriented orthogonally within the material, this special case can be described by orthotropic theory. Here the stress tensor $\sigma$ is defined by the elasticity matrix $D$ and the elastic $\left({ }^{e l}\right)$ strain tensor $\varepsilon$.

$$
\{\sigma\}=[D]\left\{\varepsilon^{e l}\right\}
$$

The compliance matrix $C=D_{-1}$ in polar coordinates is defined as:

$$
\left[D_{R \Theta}\right]^{-1}=\left[\begin{array}{ccc}
1 / E_{R} & -v_{R \Theta} / E_{R} & 0 \\
-v_{R \Theta} / E_{\Theta} & 1 / E_{\Theta} & 0 \\
0 & 0 & 1 / 2_{R \Theta}
\end{array}\right]
$$

where $G_{R \Theta}$ is the shear modulus in the $R \Theta$-plane (i.e. it represents the rigidity experienced when sliding the anterior versus the posterior cornea), $E_{\Theta}$ is the longitudinal elastic modulus (i.e. it represents the rigidity of the collagen lamellae), $E_{R}$ is the radial elastic modulus (i.e. it represents the transversal rigidity of the cornea and is related to the compressibility and extensibility of its thickness), $v_{\Theta R}$ and $v_{R \Theta}$ are the major and the minor Poisson's ratio, respectively. As $D^{-1}$ is presumed to be symmetric, the mayor Poisson's ratio results from:

$$
v_{\Theta R}=\frac{E_{\Theta}}{E_{R}} \cdot v_{R \Theta}
$$


Chapter 1. Introduction

\section{Anisotropic Properties}

Anisotropic theory describes the general and hence the most complicated case, where material properties can vary along the material in an asymmetric way. The compliance matrix is defined by 66 different parameters. Together they describe the structural organization of material properties.

\section{Models for the Cornea}

Different methods and models have been applied in order to describe the stress-strain relation in the corneal tissue.

\section{Simple Analytical Approach}

The Laplace's equation describes the capillary pressure (or pressure difference) $p$ over an interface (defined by the two radii $R 1$ and $R 2$ ) in terms of surface tension $\sigma$ :

$$
p=\sigma \cdot\left(\frac{1}{R 1}+\frac{1}{R 2}\right)
$$

The equation can be derived by the principle of minium energy or by requiring force equilibrium. In this representation an infinitely thin membrane is assumed, i.e. the thickness of the interface is no considered. In an approximation, the eye globe can be considered a thin vessel with a contained fluid where $R 1$ represents the anterior and $R 2$ the posterior radius of curvature. Considering that the cornea is weaker than the sclera (and hence corneal deformation dominates), the material properties of the vessel can be assumed to represent the corneal properties. The Laplace equation then can be applied to estimate the stress in the cornea induced by the intraocular pressure. Considering furthermore that the cornea has a finite thickness, we obtain the following equation:

$$
\sigma=\frac{R \cdot p}{2 \cdot t h}
$$

where $\sigma$ represents corneal stress; $R$ represents the mean corneal radii 
of (anterior, posterior, horizontal and vertical) curvature; $p$ represents IOP; and th represents corneal thickness. The strain on the stress produced by IOP can be expressed by

$$
\epsilon=\frac{\Delta R}{R}
$$

where $\epsilon$ represents strain, $\Delta \mathrm{R}$ represents difference in radius of curvature with respect to an initial measurement, and $R$ represents radius of curvature.

\section{Complex Analytical and Numerical Approaches}

Modelling the corneal tissue can be addressed at the level of molecular composition and interaction, at the level of collagen structure or at the level of macroscopic mechancical behavior. It is argued, which is the right level of complexity. The macroscopic response of the corneal tissue is determined by its microscopic structure, however simulating numerically the micro-structure is computationally too expensive. Studies at the molecular level (i.e. considering the interaction between proteins and sugars within the unit cell) therefore can account primarily for basic processes within the cornea, such as corneal transparency and hydration, which was addressed in a recent study modelling the interaction between stromal proteoglycans and their linear side-chains (glycosaminoglycans) [33]. Microscopic information can be included in a macroscopic model by averaging the properties across the corneal thickness and determining the corresponding material properties, which then are used as input in a finite element model. Pinsky et al [162] considered the collagen lamella structure and accounted for collagen fibrils, the extracellular matrix and proteoglycan cross-linking, which then were averaged and fitted to an anisotropic material model. In a more recent study this model was further improved, including also the interweaving of collagen fibrils along the corneal thickness [157], which has been validated with torsional shear and indentation experiments. A similar, but less detailed approach has been used by Pandolfi et al [152] and Han et al [83] assuming that the 
Chapter 1. Introduction

cornea can be described by an orthotropic or anisotropic hyperelastic material model, respectively, with a higher stiffness along lamellae and a reduced stiffness in the axial direction.

\section{Material Testing}

\section{Stress-Strain Test}

Stress-strain measurements are the Gold standard for determining the mechanical behavior (such as the Young's modulus) of materials. Typically the material specimen is stretched and the corresponding force (=stress) is measured in a strip extensiometer. The engineering stress-strain curve (where strain is calculated referring to the initial cross-section) and the true stress-strain curve (where strain is calculated referring to the current cross-section) must be differentiated. Engineering stress and strain are defined by $\sigma=\frac{F}{A_{0}}$ and $\epsilon=\frac{\Delta L}{L_{0}}$ respectively, while true stress and strain are defined by $\tau=\frac{F}{A}$ and $\epsilon=\ln \frac{\Delta L}{L_{0}}$ respectively. Figure 1.15 illustrates the

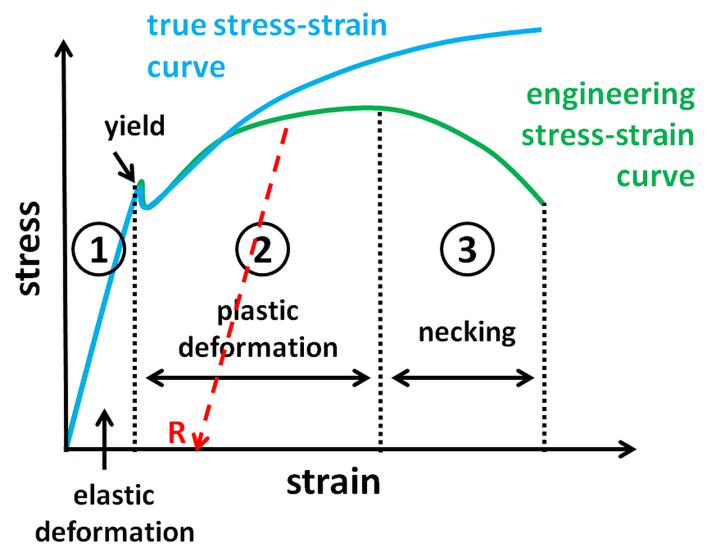

Figure 1.15: Stress-strain curves of a material showing three zones: (1) elastic deformation (Hooks law applies), (2) plastic deformation, (3) necking, with final rupture of the specimen 
two kinds of stress-strain curves. Differences are mainly observed when large deformations occur. In the very beginning (zone 1), almost every material behaves as linearly elastic and follows the Hook's law:

$$
\sigma=E \cdot \epsilon
$$

where $E$ is a constant, the elastic modulus (also Young's modulus). All deformations occurring in this zone are completely reversible. The yield stress indicates the transition from zone 1 to zone 2 . In zone 2 the material deforms permanently plastic, i.e. upon releasing stress only the elastic deformation can be recovered (indicated by point $\mathrm{R}$ ). In the necking zone (zone 3) true and engineering curves differ: The true stress-strain curve represents the real behavior of the material, as the cross-sectional area is recomputed for each strain. The curve gets steeper indicating that the material stiffens until failure. For the engineering stress-strain curve the original cross-sectional area of the specimen is considered at all strains. The apparent decrease in the necking region is because the cross-section of the material gets smaller due to excessive stretching.

Stress-strain tests are always performed within the elastic region of the material (zone 1), typically between 3\% and 15\% strain. They serve to measure the static material properties (such as the Young's modulus) of most materials. With a slightly different set-up stressstrain recording also allows to characterize viscoelastic materials. Two approaches are used: For the creep test, stress is held constant and the increasing strain with time is measured; for the relaxation test, strain is held constant and the decreasing stress with time is recorded. Figure 1.16 shows the corresponding graphs, from which the predominant visocelastic time constant $(\tau)$ can be obtained. Material creep follows the equation $\epsilon=\epsilon_{\max } \cdot\left(1-\exp \frac{-t}{\tau}\right)$ and material relaxation $\sigma=\sigma_{\max } \cdot \exp \frac{-t}{\tau}$. This means when time is equal to $\tau$, strain has reached $63 \%$ of its absolute value (creep test) and stress has reduced to $37 \%$ (relaxation test). 
Chapter 1. Introduction
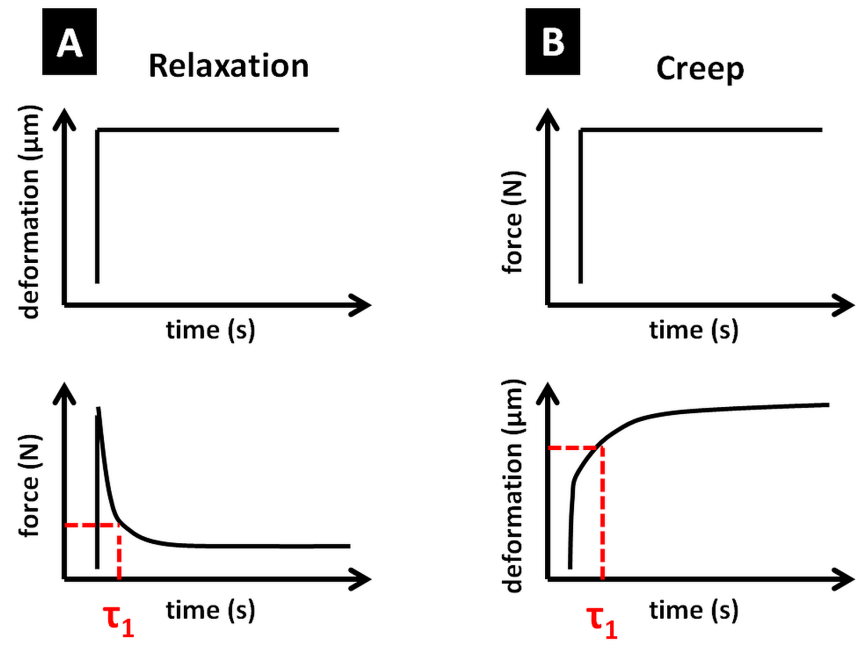

Figure 1.16: Creep and relaxation testing scheme (typical for viscoelastic materials).

Preconditioning In soft tissue, typically various loading-relaxation cycles (up to 15\% strain [124]) are performed in order to align the fibrils along the load direction and to increase the measurement repeatability prior to recording the stress-strain curves. If this procedure shall be applied with viscoelastic materials, sufficient recovery time must pass between the pre-conditioning and the stress-strain test in order not to eliminate the viscoelastic contribution.

\section{Modal Testing}

Modal testing is used to measure the dynamic behavior of materials or constructions. Studying the dynamic properties - such as the natural frequencies, mode shapes and damping - has become important in the construction of buildings and bridges in order to predict their behavior under typical load and in extreme situations (e.g. earthcakes) with the objective to make them more resistant. (A typical example where these calculations failed is the Tacoma-Narrows bridge, which collapsed due to 
resonance induced by its traffic.) The dynamic behavior of a structure is very different from its static behavior.

Modal testing is based on measuring the Frequency Response Function (FRF) of any arbitrary object. In the standard procedure vibration is induced by a tip oscillating at different frequencies. Thereby the hardness of the tip needs to be chosen in such a way that all frequencies are getting excited for a proper analysis.

\section{Dynamic Modulus}

In purely elastic materials stress and strain is completely in-phase, while in purely viscous materials the strain lags the stress by 90 . Viscoelastic materials are situated somewhere in between. Therefore a new nomenclature is introduced to describe these characteristics:

$$
\begin{gathered}
\epsilon=\epsilon_{0} \cdot \sin t \omega \\
\sigma=\sigma_{0} \cdot \sin t \omega+\delta
\end{gathered}
$$

Here $\omega=2 \pi \cdot f$, where $f$ is the frequency of the strain oscillation, $t$ is time and $\delta$ is the phase lag between stress $\sigma$ and strain $\epsilon$. Viscoelastic materials behave much more rigid, if a force is applied very quickly than if the force would be applied slowly.

$$
\begin{gathered}
E=E^{\prime}+i E^{\prime \prime} \\
E^{\prime}=\frac{\sigma_{0}}{\epsilon_{0}} \cdot \cos \delta \\
E^{\prime \prime}=\frac{\sigma_{0}}{\epsilon_{0}} \cdot \sin \delta
\end{gathered}
$$

where $E$ is the complex dynamic modulus, composed of $E$, the in-phase storage modulus (measures the stored energy, i.e. elastic, recoverable energy) and $E$ ", the out-of-phase loss modules (measures the 
Chapter 1. Introduction

energy converted into heat, i.e. energy that is not further mechanically available).

\section{Measuring Corneal Elasticity}

This section reviews literature on techniques that have been applied to measure the biomechanical properties of corneal tissue.

\section{in vitro Methods}

Most experiments assessing stress-strain curves of the cornea are done in vitro, as it is the most direct and considered a gold standard approach to measure the Young's modulus. Different approaches have been applied using different geometries, stress distributions and boundary conditions. The most common techniques are explained here:

\section{D-Strip Extensionmetry}

The most extended assessment of corneal mechanical properties have been done through 1-D strip extensiometry. In these experiments the enucleated eye is normally eviscerated and small strips (typically of 1-2 mm width) of the central cornea were obtained. The strips are subsequently mounted within appropriate clamps (see Figure 1.17) and placed in a stress-strain extensiometer.

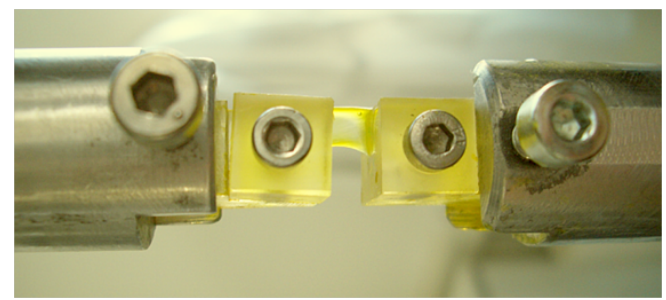

Figure 1.17: $1 D$ corneal flap mounted within clamps for uni-axial stress-strain extensiometry measurements. 
Although human corneas a generally weaker than porcine corneas, the increase in stiffness after cross-linking is much stronger in human than in porcine. Boyce et al [17] studied the general mechanical properties of the cornea confirming its viscoelastic behavior and non-linearity at higher stresses.

A limitation of uni-axial stress-strain tests in corneal tissue is that lamellae are cut during flap preparation, which changes dramatically the stress distribution along the tissue compared to the natural condition. Furthermore the initially curved tissue is flattened to a 1D-strip, inducing tensile strain on the posterior side and compressive strain on the anterior side [56].

\section{Button Inflation}

In corneal button inflation tests, the cornea and a small scleral ring are cut out of the eye globe and mounted onto an artificial pressure chamber. This approach overcomes several disadvantages of strip extensiometry: The cornea maintains its natural shape, and applying pressure to the posterior cornea results in a similar stress distribution as in the natural condition. For the measurements, the cornea is first excised from the eye globe (with a small scleral ring left) and then mounted in an artificial anterior chamber with a completely rigid fixation at the limbus. A detailed explanation of the implementation of the button inflation experiment performed in this thesis can be found in chapter 2. Stress-strain measurements have been performed, while changing the pressure within the chamber recording the full-field local deformation [16] or changes in the apex position [56].

Limitations of this technique include artificial stresses induced at the limbus due to the forced rigid boundary. The latter also leads to a different expansion behavior, compared to the natural condition. 
Chapter 1. Introduction

\section{Whole-Globe Inflation}

In whole eye globe inflation corneal expansion is studied in its natural environment, i.e. surrounded by the limbus and sclera. Typically a needle is introduced into the anterior chamber allowing to control the intraocular pressure during the experiment. A detailed explanation of the implementation of the button inflation experiment performed in this thesis can be found in chapter 2 .

Hennighausen et al [86] used this technique in combination with a confocal microscope to measure the displacement of keratocytes within the corneal tissue as a function of IOP. The anterior cornea was found to deform less than the posterior cornea, while this difference gets increased the more swollen the cornea. Asejczyk-Widlicka et al [7] performed in vivo eye inflation and measured the relation between infused volume and intraocular pressure. Whole globe inflation has also been applied to measure the local deformation by means of mercury droplets on the corneal surface [101].

Limitations of this technique include that the expansion behavior of the eye globe is a sum of corneal and scleral deformation. Hence only approximations for the corneal contribution can be made.

\section{Clinically applied in vivo methods}

Generally all in vivo techniques can also be applied in vitro. However due to their nature of being non-invasive, in vivo techniques typically asses the corneal stiffness more indirectly than in vitro techniques do.

\section{Eye inflation}

Pallikaris et al [151] performed in vivo eye inflation during eye-surgery and measured the relation between infused volume and intraocular pressure. Thereby a higher ocular rigidity was observed with age. 


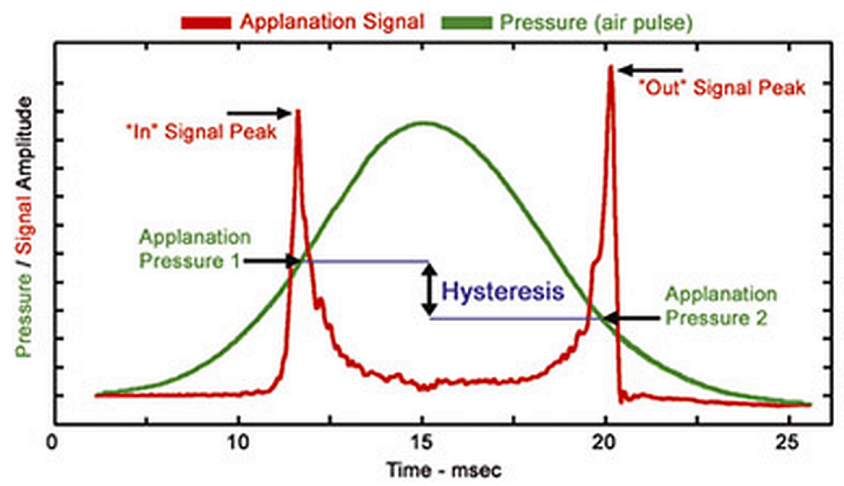

Figure 1.18: Signal recorded with the Optical Response Analyzer (ORA), image taken from manufacturer website (www.reichert.com)

\section{Ocular Response Analyzer}

The Ocular Response Analyzer (ORA) has been the first FDA approved device to measure corneal biomechanics. It is based on an air-puff emitting tube and a light sensor that measures the deviation of a laser beam (adjusted to the corneal apex) during the corneal deformation following air-puff ejection. Thereby the air-puff pressure for both corneal applanations (from inward and outward deformation) is recorded and the difference of both is defined as corneal hysteresis $(\mathrm{CH})$. $\mathrm{CH}$ is related to the viscoelastic properties of the cornea. The first and the second applanation pressure also allow the estimation of the IOP. The following formula are applied to estimate the different parameters:

- Goldmann correlated IOP: $I O P g=\frac{P 1+P 2}{2}$

- Corneal compensated IOP: IOPcc

- Corneal Hysteresis: $C H=P 1-P 2$

- Corneal Resistance Factor: $C R F=P 1-k \cdot P 2$ 
Chapter 1. Introduction

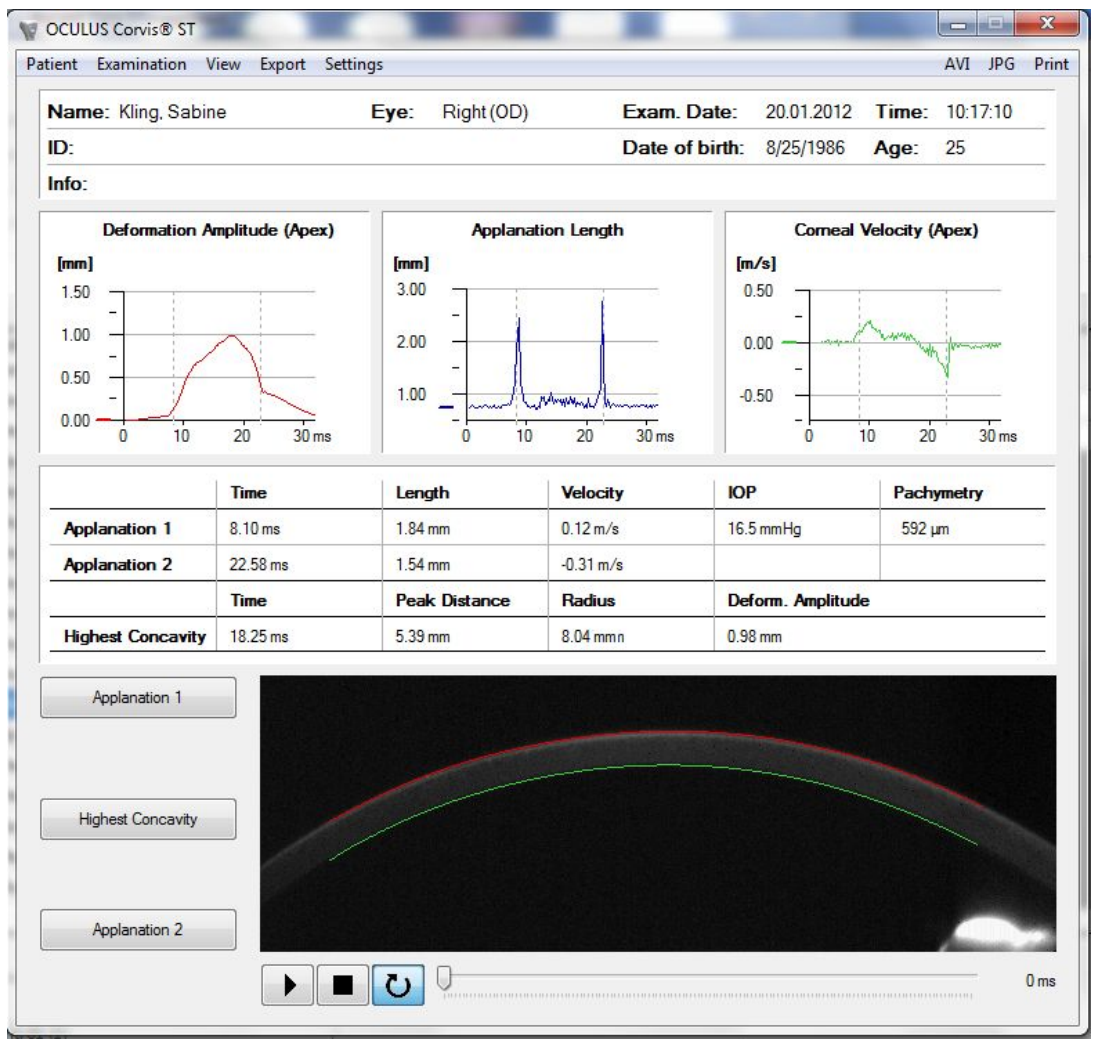

Figure 1.19: Corvis ST software. 


\section{Air-puff with Scheimpflug}

During the development of this thesis, a new commercial system has been released (Corvis, by Oculus, Wetzlar, Germany) that combines airpuff with Scheimpflug imaging high speed acquisition of horizontal crosssectional corneal images 1.19 . The system has been recently granted FDA approval as a tonometer. Prior to its commercial release the company made available to our laboratory a prototype, with a software providing quantitative information on spatial and temporal corneal deformation parameters.

\section{State of the art of experimental ocular biomechanics}

\section{Cornea}

The Young's moduli of cornea determined experimentally differ by up to two orders of magnitude. Table 1.4 hows some relevant examples. This large variation can be attributed to the different hydration conditions and the time elapsed post-mortem, but also to the different techniques used to measure the corneal stiffness in the different studies. The general trend can be observed that strip-extensiometry leads to higher Young's moduli than ultrasound or magnetic resonance based techniques.

HUMAN CORNEA The Young's modulus of human cornea is about 1.3 $\mathrm{MPa}$ in the virgin cornea and about 5.9 MPa after cross-linking (measured by 1D-strip extensiometry) [226]. The cornea is prone to have a non-linear stress-strain curve. Its central thickness is about $550 \mu \mathrm{m}$ and the physiologic intraocular pressure $15 \mathrm{mmHg}$. Models of the cornea generally consider its orthogonal fibril orientation [152] and viscoelasticity [74], while simulations mostly have been performed for static loading cases.

PORCINE CORNEA Porcine eye models are often used for in vitro experiments in ophthalmology, as human and pig corneas show a similar 
Chapter 1. Introduction

Table 1.4: Corneal Young's moduli reported in literature.

\begin{tabular}{|l|c|c|c|c|}
\hline author & year & method & specie & Young's modulus \\
\hline Wollensak & 2009 & strip-extensiometry & rabbits & $1.11 \mathrm{e} 7$ \\
Wang & 1996 & ultrasound & human & $5.30 \mathrm{e} 6 \quad 2.00 \mathrm{e} 7$ \\
Andreassen & 1980 & strip-extensiometry & human & $3.63 \mathrm{e} 7$ \\
Hjortdal & 1995 & mercury droplets & human & $3.00 \mathrm{e} 62.00 \mathrm{e} 7$ \\
Schumacher & 2009 & strip-extensiometry & porcine & $2.91 \mathrm{e} 6$ \\
Wollensak & 2003 & strip-extensiometry & porcine & $1.59 \mathrm{e} 6$ \\
Wollensak & 2003 & strip-extensiometry & human & $1.30 \mathrm{e} 6$ \\
Hamilton & 2008 & applanation tononmetry & human & $2.90 \mathrm{e} 5$ \\
Cartwright & 2011 & speckle interferometry & human & $2.70 \mathrm{e} 5 \quad 5.20 \mathrm{e} 5$ \\
Tanter & 2009 & ultrasound & porcine & $1.90 \mathrm{e} 5$ \\
Bao & 2012 & apex displacement & porcine & 7.00e4 2.90e5 \\
Litwiller & 2010 & magnetic resonance & bovine & 4.00e4 $1.85 \mathrm{e} 5$ \\
\hline
\end{tabular}

stress-strain response [233] under applied loading. Although there are some differences such as a higher corneal thickness, a lower viscoelastic creep [55] and a higher stiffening after cross-linking treatment [226], porcine eyes are considered a good model to study ocular biomechanical properties and their change in response to treatments. The water content of porcine corneas [81] is slightly lower compared to humans $(71.79 \%$ vs. $78 \%$ ) and therefore porcine corneas might be more susceptible to edema. The central corneal thickness is about $850 \mu \mathrm{m}$ and the IOP 15 $\mathrm{mmHg}$, similar to humans[172]. The Young's modulus reported is about 1.5 MPa in the virgin cornea and about 2.7 MPa in after cross-linking (measured by 1D-strip extensiometry) [226].

RABBIT CORNEA The rabbit cornea only consists of four layers: epithelium, stroma, Descemet's membrane and the endothelium. Interestingly it lacks the Bowman layer. Compared to the human eye $(23.5 \mathrm{~mm}$ diameter), the rabbit eye is much smaller $(\sim 18 \mathrm{~mm})$ although its cornea is large (covering up to $30 \%$ of the rabbit's eye) - even larger than the human cornea (nonetheless its radius of curvature is similar). Corneal 
thickness is about $420 \mu \mathrm{m}$ and the IOP ranges between 20 and $25 \mathrm{mmHg}$ [209] - slightly higher than in humans. Its Young's modulus has been reported to be $11.1 \mathrm{MPa}$ in the virgin cornea and about $20 \mathrm{MPa}$ after cross-linking (measured by 1D-strip extensiometry) [224].

\section{Sclera}

The difference between corneal and scleral stiffness varies from factor 3 (0.05-0.24 MPa versus 0.15-0.83 MPa, measured by pressure-volume relationship) [7] to factor 36 (40-185 $\mathrm{kPa}$ versus 1-7 $\mathrm{MPa}$, measured by magnetic resonance) [123]. 
Chapter 1. Introduction

48 de 329 


\section{2 \\ Methods}

In this chapter the experimental and numerical techniques used in this dissertation are described. Different optical systems were used to measure the corneal deformation under different loading conditions. The imaging systems used for this purpose were commercial instruments (Pentacam Scheimpflug, Corvis Scheimpflug), custom-developed instruments (high speed spectral OCT) available in the Visual Optics and Biophotonics Lab (Instituto de Óptica, CSIC, Madrid, Spain) and at the Wellman Center for Photomedicine at Harvard University in Boston (Brillouin microscopy, OCT vibrography).

The author of this thesis designed the experiments and implemented in collaboration with Alfonso Pérez-Escudero and Daniel Pascual (both Instituto de Óptica, CSIC, Madrid) the eye globe / corneal button mounting systems and the control of the intraocular pressure. She also developed 
Chapter 2. Methods

the analysis routines for the Corvis Scheimpflug and the iTrace ray tracing measurements and collaborated with Alfonso Pérez-Escudero in the analysis of Pentacam Scheimpflug images, with Harilaos Ginis (University of Heraklion, Greece) in the 2D-flap mounting system, with Ernest Chang and Imran B. Akca (both Wellman Center for Photomedicine, Boston) in the analysis of OCT Vibrography measurement and with Carlos Dorronsoro (Instituto de Óptica, CSIC, Madrid) in the analysis OCT air-puff images. Part of the finite element models were developed in collaboration with Nandor Bekesi (Instituto de Óptica, CSIC, Madrid). Principal investigators in of the laboratories where this research was conducted are Profs. Susana Marcos, Andy Yun and Ionanis Pallikaris, respectively. 


\section{Experimental Methods}

\section{Imaging Systems}

In this section various imaging techniques are explained, which have been used in the following chapters to measure corneal deformation under different loading conditions.

\section{Scheimpflug Imaging}

For the experiments presented in this thesis the Pentacam anterior segment imaging system from Oculus GmbH (Wetzlar, Germany) was used. In this system, the Scheimpflug camera consists of a modified slit-lamp camera placed in a certain angle to the eye. During the measurement both, slit light and camera rotate around the optical axis of the instrument, allowing the capture of 25 anterior segment sections with a lateral resolution of $56.7 \mu \mathrm{m}$ ( $141 \times 141$ pixels). From these cross-sections then $3 \mathrm{D}$-elevation maps of the anterior and posterior corneal surfaces are obtained (see Figure 2.1).

The accuracy of the anterior and posterior corneal shape measured with this system have been validated previously using a hybrid porcineplastic model eye [154]. The authors demonstrated that the optical distortions are properly corrected and that the system reliably measures anterior $(\delta=0.04 \mathrm{~mm})$ and posterior $(\delta=0.26 \mathrm{~mm})$ corneal curvature. It also was demonstrated that measurements of the posterior corneal radius of curvature and asphericity are not affected by changes in the anterior corneal surface (i.e. no correlation between both surfaces, $p>0.6$ ).[154]

\section{Optical Coherence Tomography}

SPECTRAL OCT System Figure 2.4 shows the schematic set-up of the spectral OCT (sOCT) system [77] that was used for the experiments presented in chapter 7 . 
Chapter 2. Methods

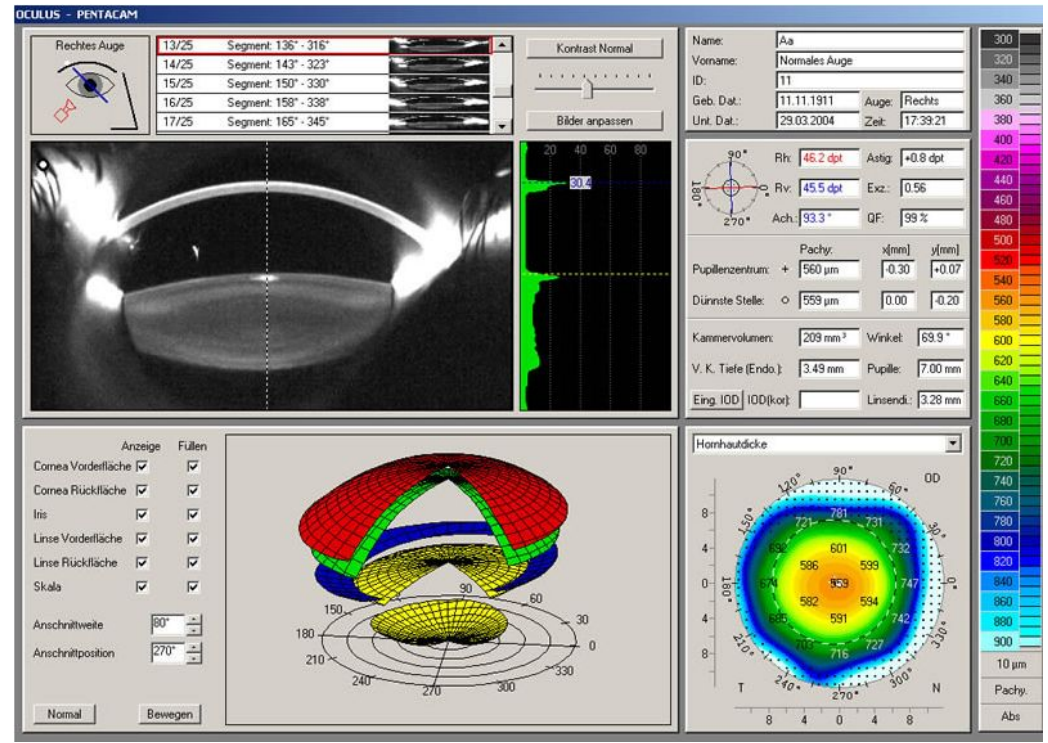

Figure 2.1: Commercial Pentacam Scheimpflug system.

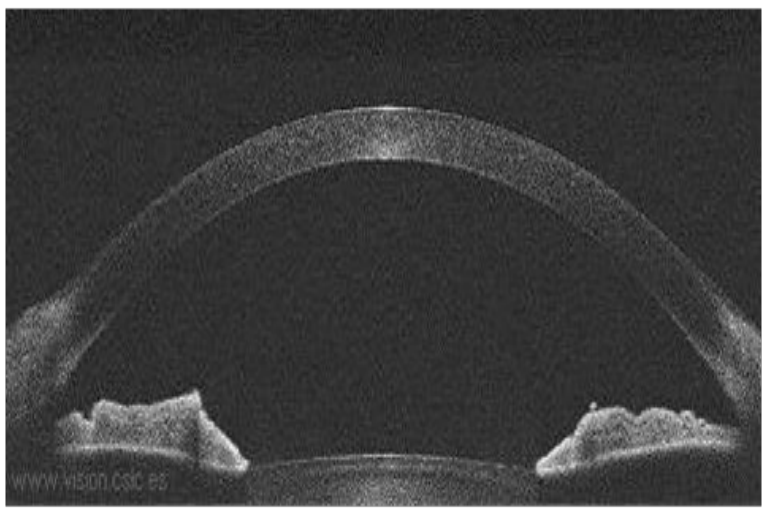

Figure 2.2: Typical image of a human cornea obtained with the sOCT system. 
The system has been developed at Instituto de Óptica (CSIC) in collaboration with the Copernicus University (Toruń, Poland). A sketch of the spectral-domain OCT is shown in Figure 2.3. The system uses a superluminescent diode laser source (centered at $840 \mathrm{~nm}$; Superlum, Ireland) and a spectrometer with a line-scan camera to record the spectral fringe pattern. This fringe pattern is then Fourier transformed in order to the separate the signals comming from different depths (different wavelengths within the $50 \mathrm{~nm}$ bandwidth of the light source are reflected at a different layers of the scanned sample).

A typical static image of the anterior segment of the eye obtained by this system is shown in Figure 2.2.

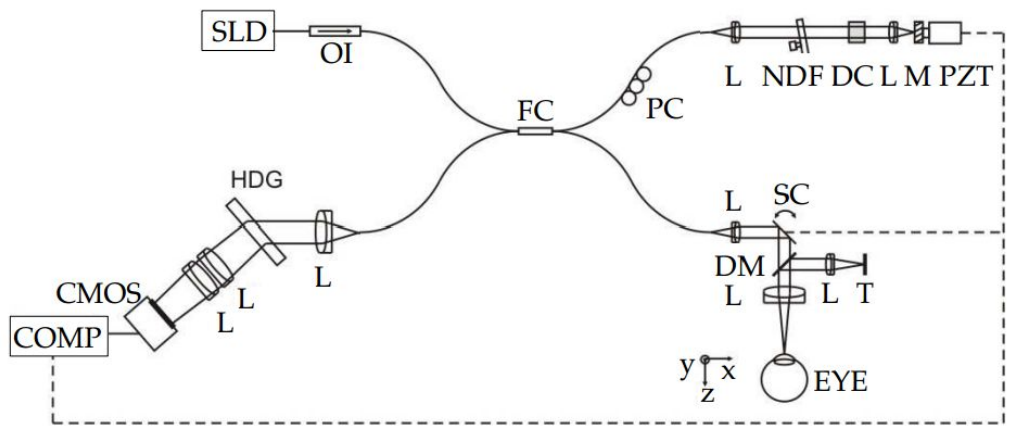

Figure 2.3: Sketch of the SOCT setup: SLD - superluminescent diode, OI - optical isolator, FC - 80:20 fiber coupler, PC polarization controller, NDF - neutral density filter, DC dispersion compensator, L1-L8 - lenses, $M$ - silver mirror, PZT - piezotranslator, SC - galvanometric scanners, DM - dichroic mirror, $T$ - target, HDG- holographic volume diffraction grating, CMOS - linescan camera, COMP computer. (Reproduced from [77])

The signal to noise ratio is $97 \mathrm{~dB}$ and the acquisition speed 25000 A-Scans/s. The axial range of the instrument is $7 \mathrm{~mm}$ in depth, resulting in a theoretical pixel resolution of $3.42 \mu \mathrm{m}$ in air. The axial resolution predicted by the $50 \mathrm{~nm}$ bandwidth of the laser source is $6.9 \mu \mathrm{m}$. The superluminiscent diode power was set to $1.5 \mathrm{~mW}$ for the in vitro measurements, and to $1.0 \mathrm{~mW}$ for the in vivo measurements. 
A single 2-D image to capture the corneal deformation following an air-puff consisted of $90 \mathrm{~A}$-scans in a lateral range of $15 \mathrm{~mm}$, leading to a spatial sampling of $0.17 \mathrm{~mm}$ and a temporal sampling of $1.35 \mathrm{~ms}$. A single 1-D image of the apex deformation along time consisted of a consecutive scan of A-lines without moving the scanner. The acquisition time of the camera (12-bit line-scan CMOS with 4096 pixels; spL4096-140k by Basler AG, Germany) was set to $15 \mu \mathrm{s}$.

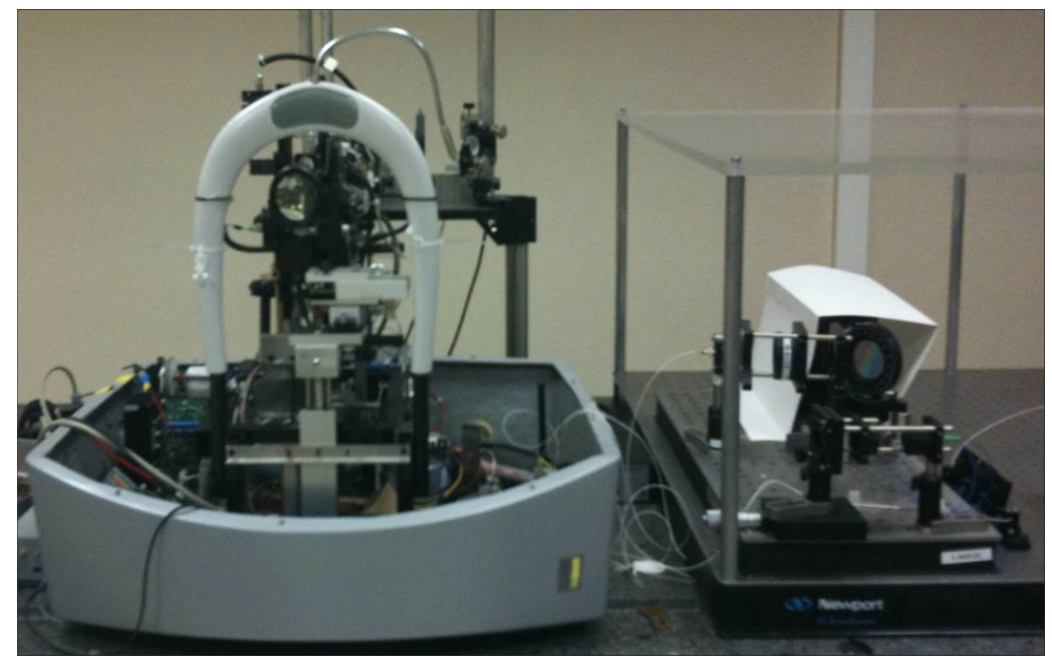

Figure 2.4: sOCT system.

Ortiz et al [146] have developed fan and optical distortion correction algorithms which allowed the captured images to be quantitatively analyzed. Fan distortion (affecting the radius of curvature by about 3\%and asphericity by about $125 \%$ ) arises from the scanning architecture of the OCT system (separation of scanning mirrors and aberrations of optical components, the collimator lens in particular). It can be minimized by hardware and fully corrected by software using ray tracing. Optical distortion arises from viewing the internal optical surfaces through refracting preceding surfaces. After distortion correction the radius of curvature and asphericity of an artificial lens could be measured with 
$>90 \%$ accuracy (compared to non contact profilometry) [148].

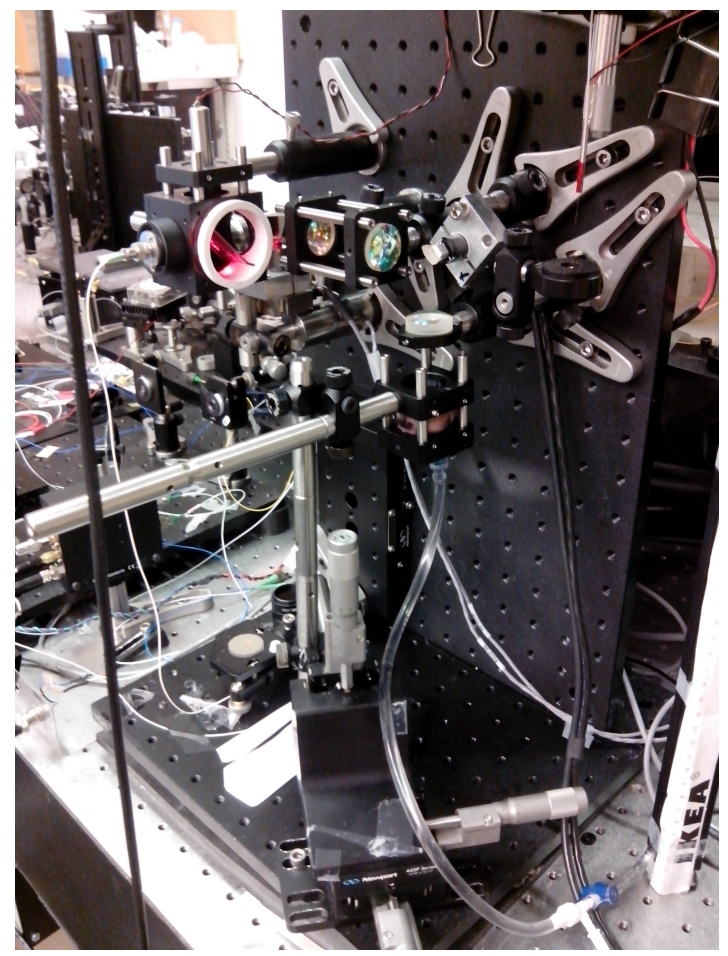

Figure 2.5: Phase-sensitive swept-source OCT system.

Phase-Sensitive SWePt-source OCT System Figure 2.5 shows the schematic set-up of the swept-source OCT system that was used for the experiments presented in chapter 10. It is an updated version of the system presented by Chang et al in 2012 [30]. The frequency domain OCT is based on a wavelength swept source $\left(\lambda_{0}=1280 \mathrm{mn}, \delta \lambda=125\right.$ $\mathrm{nm}$ ) with an output power of $40 \mathrm{~mW}$. The system was operated at a A-line rate of $48 \mathrm{kHz}$ and a sensitivity of $100 \mathrm{~dB}$. A multifunctional board (DAQ; PCI 6115, National Instruments) was used to acquire the interference data, to control a two-axis galvanometer beam scanner and to trigger the signal of the function generator (generating a sound wave). In this 
way the data acquisition, the beam scanning and the sample oscillation were synchronized. The lateral scanning velocity was dependent on the sampling rate of points per oscillation period at one location.

\section{Ray Tracing Aberrometry}

The iTrace system (Tracey Technologies Corp, Houston, USA) was used in the measurements presented in chapter 5 .

The ray-tracing aberrometer (see Figure 2.6) consists of a laser beam (785 nm), which is directed (parallel to the line of sight) onto a given point on the cornea within the pupil. It then analyzes by retro-reflected light the exact position where the beam hits the retina. This process is repeated for 63 more points across the cornea resulting in a lateral resolution of about $1 \mathrm{~mm}$. Then 27 Zernike polynomials are fitted to describe the ocular aberrations.

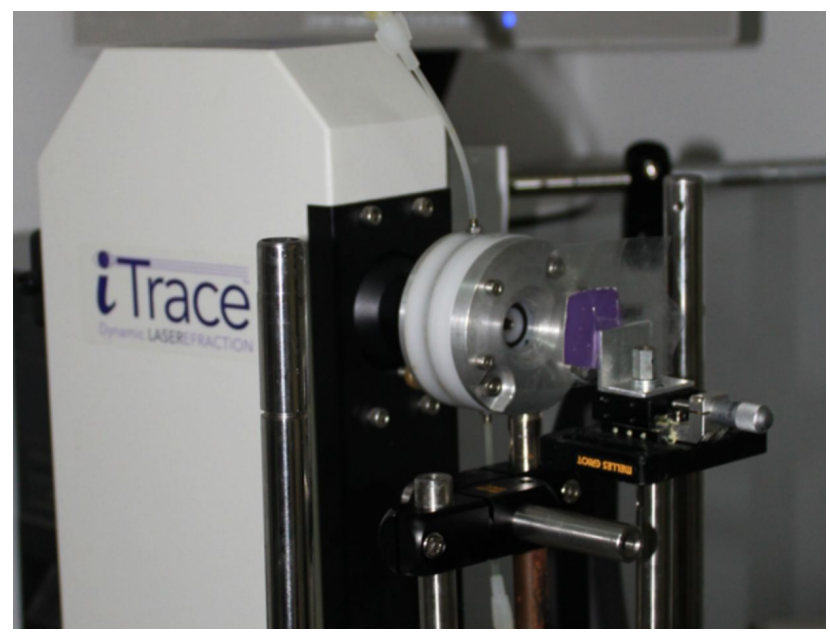

Figure 2.6: Commercial ray tracing system (iTrace). 


\section{Brillouin Microscopy}

The Brillouin microscope platform used for the experiments presented here was similar to the one described by Scarcelli et al [177] and set up in Prof. Andy Yun's lab at Wellman Center of Photomedicine (MGHHarvard). It consisted of a confocal microscope with numerical aperture (NA) of 0.3 and a high-resolution optical spectrometer. A single-mode laser at a wavelength of $532 \mathrm{~nm}$ (Torus, Laser Quantum, Inc.) illuminated the sample with a typical power of $7 \mathrm{~mW}$. The lateral and axial resolutions were approximately $1 \mu \mathrm{m}$ and $8 \mu \mathrm{m}$, respectively. 3D translation of the sample was enabled mounting it on a motorized stage (Prior Scientific). The scattered light from the sample was collected by a single-mode optical fiber (Thorlabs) and coupled into a two-stage VIPA spectrometer in the cross-axis configuration with sub-GHz frequency resolution [174, 179]. The dispersed optical spectrum was recorded with an EM-CCD camera (Ixon Du197, Andor) with a frame integration time of 200 ms. The CCD data were processed to determine the Brillouin frequency shifts by using a custom-written MATLAB program. Brillouin images were produced by plotting the measured frequency shifts over space with color encoding.

\section{Air-puff Systems}

These systems are designed to capture corneal deformation following an air-puff in order to measure the IOP and the dynamic bio-mechanical properties of the cornea. Measurements were performed on a custom built and on a commercial air-puff system, which are described below.

sOCT A commercial non-contact tonometer (NT 2000, Nidek, Hiroishi, Japan) was combined with a custom spectral domain OCT system (see chapter 2) to provide dynamic images of the corneal deformation event during an air puff. The non-contact tonometer provides an air puff which is directly impacting the cornea and produces a fast $(\sim 20 \mathrm{~ms})$ deformation event, identical to that used in non-contact corneal tonometry. A tilted mirror is attached to the tube tip providing the air puff (see Figure 2.7 for 
a schematic diagram of the setup) to allow acquisition of sOCT images.

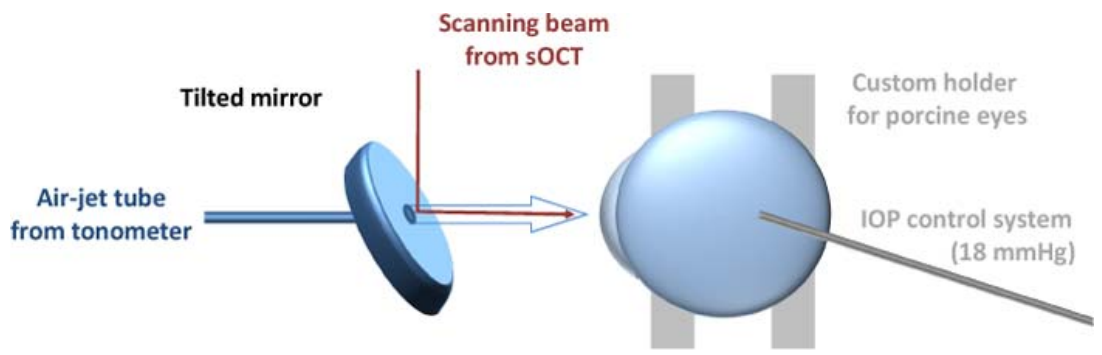

Figure 2.7: Set-up of the custom built sOCT air-puff system (bird's view). A tilted mirror is fixed to the tube tip of a modified non-contact air tonometer and provides an optical channel to a custom sOCT instrument (see text for details and angles). A custom eye holder and intraocular pressure control system (both in gray) were used for the in vitro experiments; in vivo experiments were performed without any further eye fixation.

The implementation and use of the custom-developed high-resolution sOCT instrument is described in detail elsewhere $[38,40]$. The acquisition time of the CMOS camera was set to $15 \mu$ s to provide high speed imaging of the cornea. Two configurations of the sOCT instrument were used to capture the dynamic behavior of the cornea: (1) 1-D measurements at full temporal resolution (every $15 \mu$ s) on the corneal apex, i.e. continuous A-scan recordings without moving the scanner; and (2) 2-D dynamic measurements (B-scans) of the horizontal meridians of the cornea. The lateral range of the B-scans was $15 \mathrm{~mm}$, sampled with $90 \mathrm{~A}$-scans. With this configuration, the cornea was spatially sampled every $0.17 \mathrm{~mm}$, and each corneal point was temporally sampled every $1.35 \mathrm{~ms}$. The tube tip of the non-contact tonometer was placed at 11-mm from the eye, similarly to the configuration for corneal tonometry, to ensure a standard air puff on the cornea. The air puff configuration was set to the same level in all experiments ("60 Manual" according to the denomination of the instrument used). The corneal deformation sensors of the tonometer were deactivated. In this configuration each air puff is released with the highest air pressure provided by the instrument. The tonometer 
measurement button was electronically controlled to provide a remote measurement trigger. The air tube tip of the instrument was rebuilt to allow the fixation of a mirror. Figure 2.7 shows the patient view of the system.
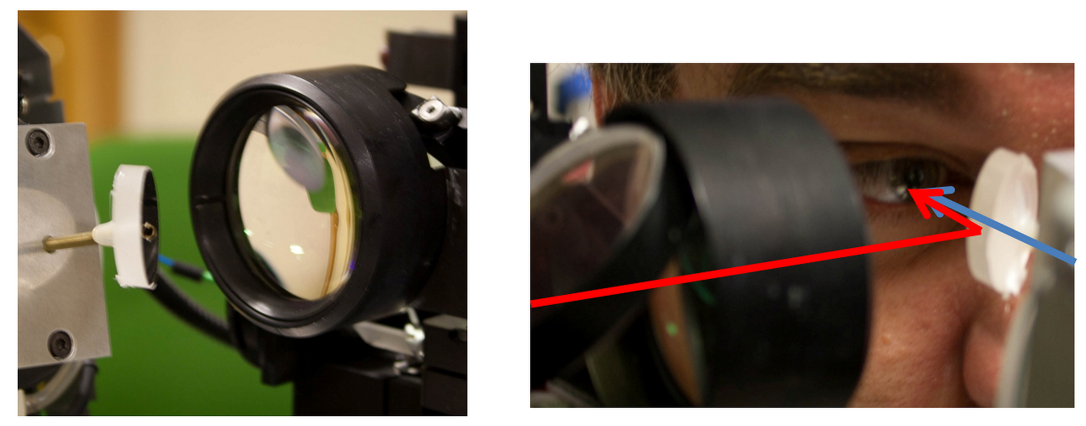

Figure 2.8: Patient view of the sOCT air-puff system. Air (blue) and laser (red) paths during the air-puff measurement by sOCT.

The mirror has a tilt angle in the horizontal direction of $45^{\circ}$ with respect both to the tonometer and the sOCT instrument, to allow both instruments to point at the corneal apex (see Figure 2.7). The mirror has an additional tilt of $8^{\circ}$ with respect to the vertical direction, which prevents the horizontal scanning beam of the sOCT from vignetting by the hole in the mirror that allows the air puff to reach the cornea. These mirror angles were set by the mirror drilling angle, produced at a precision mechanical workshop. As a result of this geometrical configuration, there is a small angle $\left(8^{\circ}\right)$ between the sOCT measurement beam axis and the air puff direction when both point at the corneal apex. In the experiments, the measurement (imaging) axis was used as a reference for the alignment of the eyes (both human in vivo and porcine in vitro). Although the resulting slightly non-normal incidence of the air puff could potentially reduce corneal deformation, this reference is preferred, since it does not induce additional distortion in the images, maintaining the symmetry of the deformation in the scanning direction (horizontal 
meridians). During the deformation, the air puff gets slightly decentered with respect to the corneal center defined by the imaging axis, but only $140 \mu \mathrm{m}$ per axial millimeter of corneal displacement. This shift can be considered negligible in comparison with the much wider corneal area in which the air pulse applies a constant force. The current configuration in which the tube tip is attached to the mirror produces slight vibrations during the air-puff release (reduced by absorbing elements disposed in the instrument holder). However, we have not observed any effect of those on the measurements during the deformation event.

SCHEIMPFLUG A recently developed air-puff system (Corvis, Occulus, Wetzlar, Germany) was used for the experiments. The Corvis system is composed of an air compressor emitting a controlled air-puff on demand and simultaneously an ultrafast Scheimpflug camera that captures 140 corneal images during the air puff event (lasting approximately $20 \mathrm{~ms}$ ). The eye is positioned in front of the system, at a distance of $11 \mathrm{~mm}$ between the corneal apex and the air tube. When the eye is aligned and the Scheimpflug image is in focus, the air-puff gets automatically released and the deformation imaged. Alternatively the air-puff can also be emitted manually. As the entire measurement happens faster than the blink reflex, in vivo measurements are minimally affected by motion.

\section{Pressure System}

During the experiments the IOP was controlled by means of a water column and a pressure (transducer)sensor (SSCM3175GA, Sensortechnics, Germany). Figure 2.9 shows the experimental set-up. The eye was connected through a needle with the water column, the pressure sensor and the syringe. The syringe was fixed on top of a customized metal block and could be moved by an external motor (NE-500, New Era Pump Systems, Inc., Wantagh, NY), which was controlled by custom routines written in Matlab. The current pressure in the eye was recorded constantly and given as input for the Matlab sub-routines over a USB 
port. In order to change the intraocular pressure, the motor was activated through the software and stopped as soon as the pressure reached the desired value. An regulatory loop was included to adjust the pressure with more accuracy. As soon as the pressure was adjusted, the imaging software was automatically called and the measurement was released. Before first time usage a calibration was performed to verify the linearity of the pressure sensor.

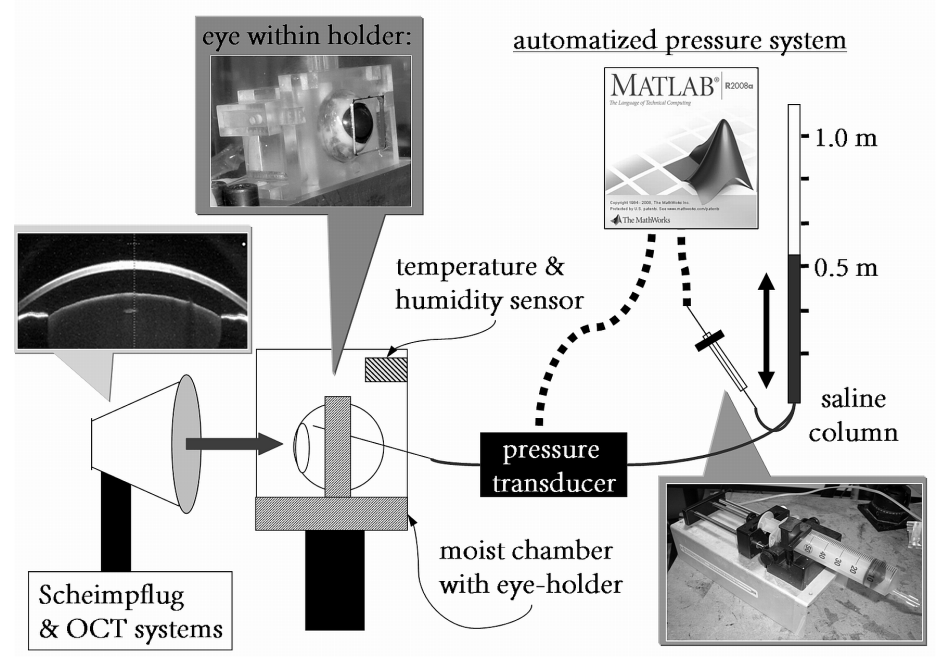

Figure 2.9: Set-up of the pressure control system.

\section{Wet-Chamber}

The eyes were placed within a custom built moist chamber (see Figure 2.10) during the measurements in order to prevent dehydration. The chamber consisted of a methacrylate box in which wet cotton cloths provided a constantly humid atmosphere. The humidity (50\%) and temperature $\left(24^{\circ}\right)$ within the chamber were continuously recorded by the manufacturer software. A window at the front side of the box allowed an unrestricted view of the eye by the imaging system. 
Chapter 2. Methods

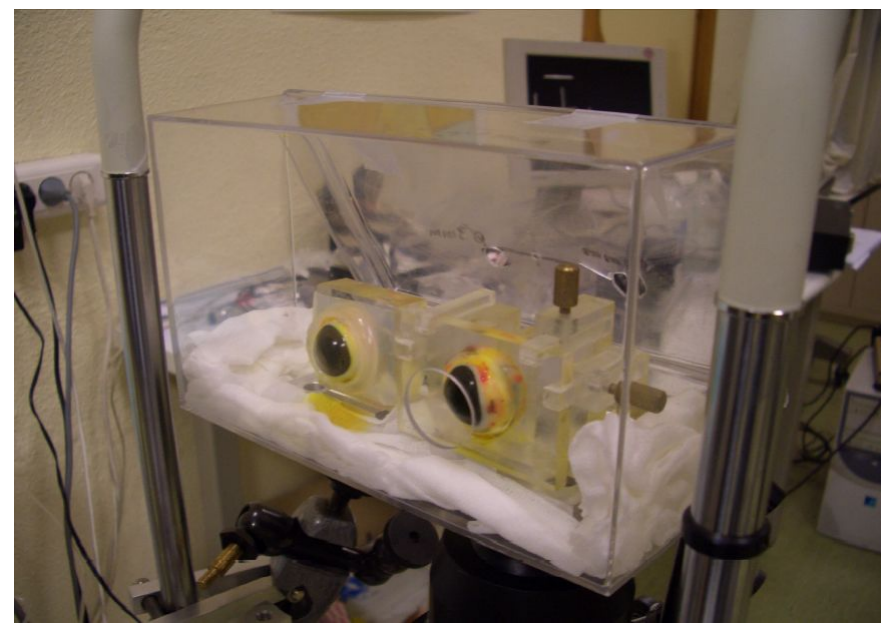

Figure 2.10: Porcine eyes mounted within the wet chamber in order to prevent dehydration.

\section{Eye-Holders}

Custom eye-holders were built to fix the eyes and corneas during the experiment in order to prevent global motion.

\section{Whole Eye Globes}

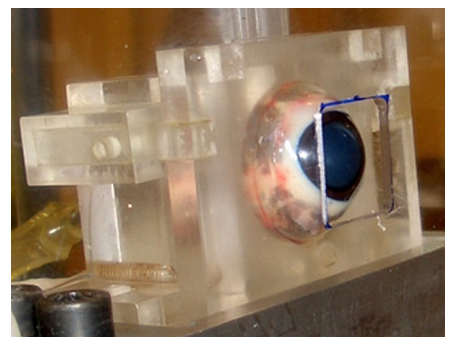

Figure 2.11: Whole eye globe holder.

A view of the holder for whole eye globes can be seen in Figure 2.11 (left). The holder consists of two rectangular plastic (Polymethylmethacrylate, PMMA) parts, each with an in-wrought half sphere of 20 
mm diameter. The distance between the plastic parts can be regulated by means of screws allowing the holder to adapt to the different size of individual porcine eyes.

\section{Corneal Buttons}

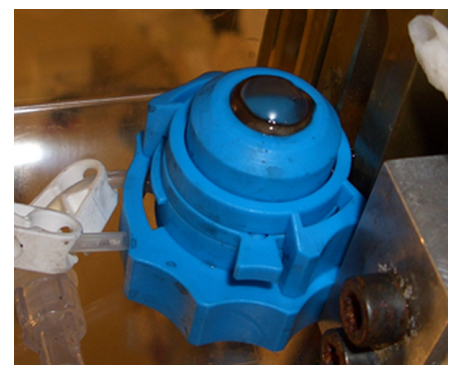

Figure 2.12: Corneal button holder.

An anterior chamber (see Figure 2.12 (right)) was used to fix corneal buttons. The commercial device (Barron Precision Instruments, L.L.C.) had an opening of $12.5 \mathrm{~mm}$ and was adequate for human buttons. We designed an additional custom holder with a wider opening $(14.0 \mathrm{~mm})$ to fit porcine eyes and fabricated it in the workshop of our center (CSIC, Madrid).

\section{D-Flap Holder}

As part of this thesis a custom flap holder (see Figure 2.13) was built to fix the two-dimensional corneal flaps created by a microkeratome. The holder consists of two chambers being separated by the flap and filled with liquid - thereby allowing stress-strain tests: Chamber 1 was filled with $0.125 \%$ Riboflavin-20\%Dextran (permitting diffusion of the photosensitizer into the flap) and connected to a pressure-system in order to apply a normal surface load onto the flap. Chamber 2 was filled with Oxane 1300 Silicon-Oil (Bausch\& Lomb) in order to preserve corneal hydration and because of its high refractive index (1.5). This chamber 
Chapter 2. Methods

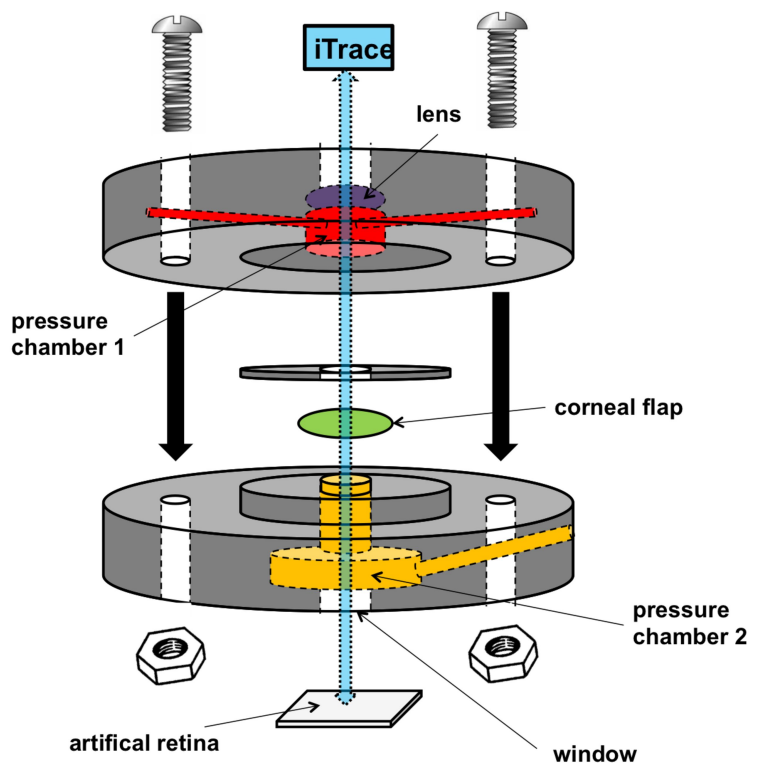

Figure 2.13: 2D Flap holder. 
was left open in order to provide atmospheric pressure independent of pressure in Chamber 1. The Dextran in the Riboflavin-Dextran solution in chamber 1 regulated the flap's hydration while the silicon oil prevented water evaporation. Under these conditions flap hydration was maintained constant throughout treatment and measurements.

\section{Microkeratome}
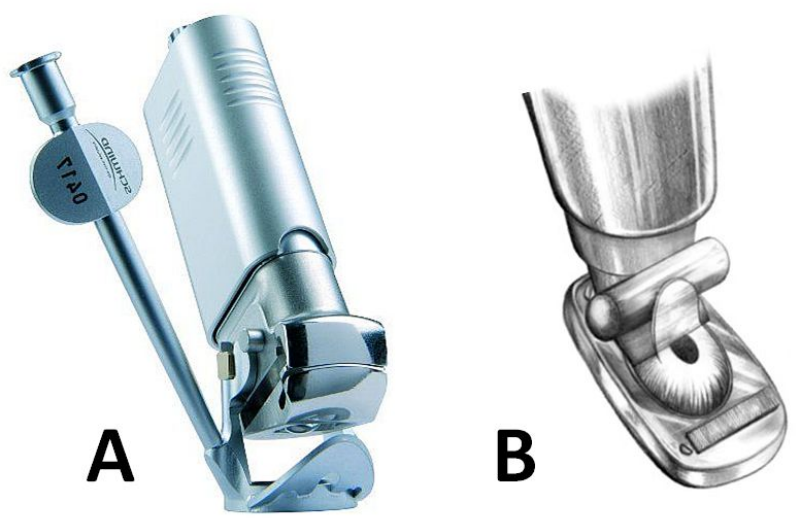

Figure 2.14: Carriazo-Pendular microkeratome.

Microkeratomes are typically used to create corneal flaps for the LASIK procedure. I this thesis we used a mechanical Carriazo-Pendular microkeratome (Schwind, Germany), see Figure 2.14A to create corneal flaps for extensiometry measurements (see chapter 5). The CarriazoPendual instrument consists of a cutting head with interchangeable blades, which performs a pedulum motion to cut the flap (at a velocity of $2-5 \mathrm{~mm} / \mathrm{s}$ ), and a suction ring connected to a vacuum chamber in order to fix the cornea to the instrument. The cutting heads are available from 90 to $170 \mu \mathrm{m}$ with a standard deviation of $10-12 \mu \mathrm{m}$. Figure $2.14 \mathrm{~B}$ shows how flaps are cut in a simplified set-up. For the experiments presented in chapter 10 a custom developed microkeratom (Deriva, Valencia) was used with a similar working principle, except that the cutting blade was 
Chapter 2. Methods

operated manually.

\section{Eyes}

\section{Porcine Eyes}

For the experiments typically fresh enucleated eyes from six-months old pigs were obtained from a local slaughterhouse (Cárnicas Vaquero, Madrid) within 4 hours post-postmortem. A total of 110 porcine eyes were used in this thesis.

\section{Rabbit Eyes}

For the experiments in chapter 5 New Zealand rabbits were obtained from a certified farm at the age of 3 months (approx. $2 \mathrm{~kg}$ weight) and studied during one month. A total of 5 rabbits were used in this thesis.

\section{Human Eyes}

Human eyes were used to verify the experimental behavior observed in the animal models (in vitro) and to study the effect of the ocular muscles (in vivo) in chapter 8. Human donor eyes were obtained through an agreement between the Fundación Universidad Autónoma de Madrid and the Instituto de Óptica (CSIC). Human patients (25 to 53 years) were normal volunteers and signed an informed consent after receiving an explanation regarding the nature of the study. All protocols followed the Tenets of the Declaration of Helsinki and had been approved by the Institutional Review Boards.

\section{Storage}

The eyes were wrapped hermetically with aluminum foil and stored at $4^{\circ} \mathrm{C}$ in a refrigerator. 


\section{Corneal Treatments}

\section{UV-Collagen Cross-linking}

As mentioned in chapter $1 \mathrm{CXL}$ is applied to increase the corneal stiffness. This section describes the treatment protocols used in this thesis. In most measurements of this thesis the standard protocol has been applied, only chapter 6 shows a comparison of both techniques.

StANDARd DRESDEn Protocol According to the protocol by Spoerl et al [195], in a first step the corneal epithelium is removed by gentle scraping with a blade or hockey knife. Then $0.125 \%$ Riboflavin-20\%Dextran solution is applied drop-wise on top of the cornea every 3 minutes for a total of 30 minutes. In a second step, the cornea is irradiated with UV-light (365 nm, $3 \mathrm{~mW} / \mathrm{cm}^{2}$ ) for further 30 minutes, while dropping Riboflavin-Dextran solution at 3 minutes intervals is continued.

We used different systems for UV irradiation $\left(365 \mathrm{~nm}, 3 \mathrm{~mW} / \mathrm{cm}^{2}\right)$, including the standard UV-XTM delivery system (Peschke; IROC, Zurich) in chapter 3, 7, 8 with an irradiation zone of $10 \mathrm{~mm}$ or UV-lamps with the same characteristics but an irradiation zone covering the entire cornea in chapter $5,6,10$. Before treatment the irradiance was measured with a calibrated UVA meter and the intensity was regulated to $3 \mathrm{~mW} / \mathrm{cm}^{2}$ with a potentiometer if necessary.

Transepithelial Corneal Collagen Cross-linking protocol The epithelium-on (epi-on) procedure was suggested by Wollensak et al [223] in order to avoid the epithelium abrasion, making the treatment more comfortable and reducing the risk of infections. The difference to the standard protocol lies in the $0.125 \%$ Riboflavin solution, which here is based on PBS without dextran. Furthermore, the solution contained an epithelium-loosening agent, $0.02 \%$ benzalkonium chloride (BAC), and optimized osmolarity ( $\mathrm{NaCl} 0.44 \%$ ) according to the protocol by Raiskup et al.[164]. Similarly, corneas are then pre-soaked with the solution for 
Chapter 2. Methods

$30 \mathrm{~min}$ and then exposed to the UV light $\left(£ \mathrm{f}=365 \mathrm{~nm}, 3 \mathrm{~mW} / \mathrm{cm}^{2}\right)$ for further $30 \mathrm{~min}$.

\section{Hydration Conditions}

Medical solutions typically used to perform CXL or to store donor eyes in eye banks were studied on their effect on corneal hydration and its biomechanics.

DEXTRAN / RiBOFLAVIN-DEXTRAN TREATMENT Dextran was applied for a short period of time (30 minutes), as it is typically done to dehydrate corneas of eye-bank eyes[233] or with Riboflavin-Dextran solutions in the cross-linking treatment.[194] De-epithelization to allow the solutions to better diffuse into the cornea was achieved with a hockey epithelium removal knife. Different concentrations of Dextran solutions were tested: 8\%-Dextran, 20\%-Dextran, and 0.125\%-Riboflavin-20\%-Dextran (which is the photosensitizer solution typically used in cross-linking treatments) in different sets of eyes. All solutions were diluted in a $0.9 \%-\mathrm{NaCl}$ solution. Solutions were instilled for 30 minutes (one drop every 3 minutes) before measurements were started.

OPtisol-GS TREATMENT Optisol GS is used specifically for long-time storage of corneas. Therefore eyes treated with Optisol GS were only measured 24 hours after storage. For treatment the eyes were immersed in Optisol GS solution overnight and stored at $4{ }^{\circ} \mathrm{C}$. In order to allow proper corneal imaging it was necessary to de-epithelialize the corneas before starting the measurements.

VIRGIN EYES Virgin eyes were used as control eyes, without undergoing any treatment. Epithelial transparency loss prevented measurements of the intact eyes after 24 hours. For this reason, the eyes were deepithelialized immediately after the first sessions. Then the eyes were wrapped hermetically with aluminum foil and stored for 24 hours before repeating the measurements.

68 de 329 


\section{Analysis Routines}

\section{Air-puff Deformation}

FROM OCT IMAGING The geometrical deformations of the cornea observed in air-puff measurements performed with the sOCT system were analyzed by extracting the parameters shown in Figure 2.15. Thereby Figure 2.15a shows the dynamics of the apex (A-scans) throughout time and Figure 2.15b depicts the corneal meridians (B-scans) before and during the deformation.

(a) Temporal dynamics of the corneal apex

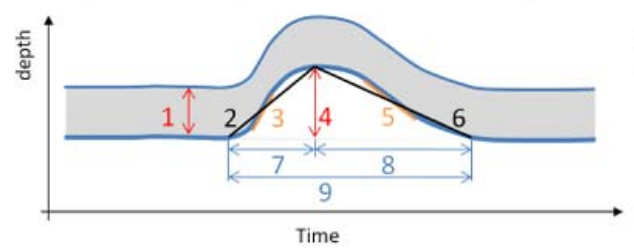

(b) Dynamics of corneal meridian

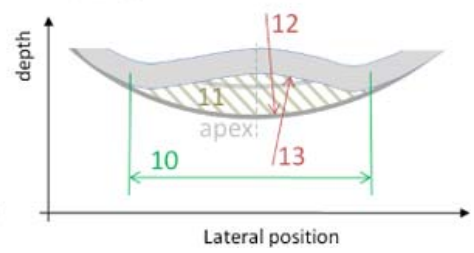

Figure 2.15: Diagram of the images resulting from the measurements and the corresponding extracted parameters. (a) Measurements of the temporal dynamics of the apex $(A-$ scans) and (b) Measurements of the dynamics (B-scans) of a corneal meridian. 1: Corneal thickness. 2: Mean deformation speed during the increasing deformation period. 3: Peak speed (maximum slope) of the increasing deformation period. 4: Deformation amplitude (peak deformation, dividing the periods of increasing and decreasing deformation). 5: Peak speed of the decreasing deformation period. 6: Mean speed of the decreasing deformation period. 7: Duration of the increasing deformation period. 8: Duration of the decreasing deformation period. 9: overall duration of the deformation event. 10: Diameter of the deformed zone. 11: Displaced volume. 12: Non-deformed corneal radius. 13: Deformed corneal radius. See text for details.

The image pixels where re-scaled in the vertical direction to provide absolute values of depth (in millimeters), and in the horizontal direction to obtain absolute values of time (Figure 2.15a; in milliseconds) and axial position (Figure 2.15b; in millimeters). Custom routines were developed to extract quantitative parameters from the captured set of 
images. The analysis is based on a direct comparison between the stable non-deformed cornea immediately before the deformation event, and the corneal deformation during the air puff. The corneal thickness ( 1 , red) is obtained as the axial distance between the anterior and posterior surface of the cornea, evaluated at the corneal apex before the deformation, during maximum deformation, and immediately after the deformation. The deformation amplitude $(4$, red) at the corneal apex represents the maximum deformation depth. This instant of maximum deformation (corresponding to the moment when the deformation stops increasing and starts decreasing, i.e., when the cornea starts recovering) divides the overall deformation (9) event in two intervals: the increasing deformation period (7) and the decreasing deformation period (8). The mean increasing and decreasing deformation speeds ( 2 and 6, black) are obtained as the deformation amplitude divided by the duration of the corresponding increasing or decreasing deformation period (slopes of the black lines in Figure 2.15a). The peak deformation speeds ( 3 and 5, orange) are also obtained for the increasing and decreasing deformation periods (as the maximum positive and negative slopes of the deformation curve, represented as orange lines in Figure 2.15a). The parameters above were obtained from high-speed A-scan measurements at the corneal apex throughout (Figure 2.15a). Additional information could be extracted from the dynamic B-scan measurements (along the horizontal meridian; Figure 2.15b). This imaging mode provided a direct view of the spatial deformation of the cornea. Although this corneal meridian measurement mode could potentially provide similar results about the apex dynamics than the A-scan measurements, the temporal sampling achievable is much lower and therefore the extracted parameters have lower precision. The B-scans were used to obtain the diameter of the deformed zone (10, green), the displaced corneal volume at the moment of peak deformation (11, gray), the non-deformed corneal radius (12) and the peak deformed corneal radius (13). 
A

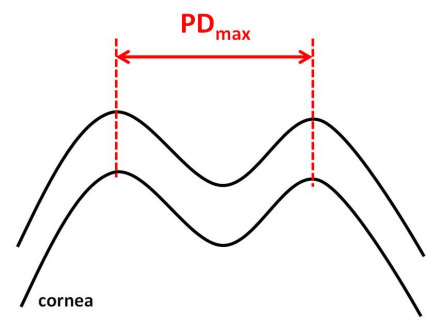

B

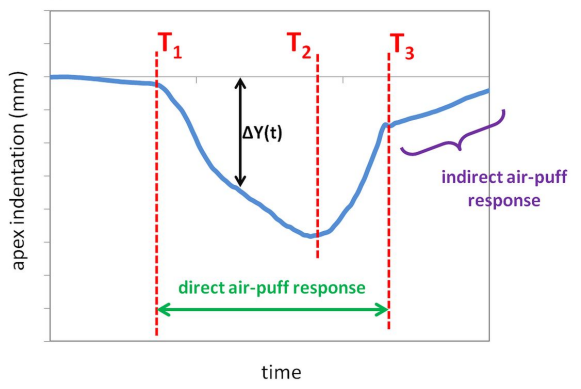

Figure 2.16: (A) Illustration of the corneal profile at maximal deformation and definition of the peak-to-peak distance (PDmax). (B) Illustration of the apex indentation as a function of time, and definition of the parameters of the temporal symmetry (Tsym), elastic air-puff response and the viscoelastic recovery and/or muscle damping.

From Scheimpflug IMAGING The geometrical deformations of the cornea observed in air-puff measurements performed with the CORVIS system were exported directly from the CORVIS software. Corneal apex indentation as a function of time and the corneal profile at maximal deformation were analyzed. The following parameters were obtained from this deformation data: (1) Peak to Peak distance $\left(P D_{\max }\right)$, i.e. the lateral distance between the two convex peaks in the corneal profile at maximal deformation (Figure $2.16 \mathrm{~A}$ ); (2) Temporal symmetry factor $\left(T_{\text {sym }}\right)$, that describes the degree of symmetry of the corneal apex versus time function (Figure $2.16 \mathrm{~B}$ ):

$$
\tau_{\text {sym }}=\frac{\sum_{T_{1}}^{T_{2}} \Delta Y_{\text {apex }}}{\sum_{T_{2}}^{T_{3}} \Delta Y_{\text {apex }}}
$$

where $T_{1}$ is the time where the deformation starts, $T_{2}$ is the time at maximal deformation, and $T_{3}$ is the time when the elastic deformation finishes. $\triangle Y_{\text {apex }}$ is the indentation of the corneal apex recorded by the Corvis system. Tsym indicates the degree of temporal symmetry in the deformation $\left(T_{\text {sym }}=1\right.$ means temporally purely axis-symmetric 
deformation). (3) Elastic air-puff response, which describes the corneal deformation as direct response to the applied air-puff, i.e. as long as the air-puff applies a direct force onto the cornea (indicated by time points $T_{1}$ and $T_{3}$ ). (4) Viscoelastic recovery and ocular muscle damping were defined as the remaining deformation after $T_{3}$, i.e. after the elastic air-puff response. Viscoelastic recovery was analyzed in all conditions. The ocular muscle damping only applies to the in vivo condition.

\section{D-Flap Geometry}

The geometric deformation observed in 2D-flap extensiometry measurements were monitored with a ray-tracing aberrometer. The amount of defocus in the artificial eye is related to the stretching of the corneal flap. The higher the pressure in Chamber 1, the more curved the flap and the higher the change of the system's refractive power. Due to small deformation produced by the applied pressures, it can be assumed that the flap is deformed spherically. This approximation is further justified as we selected a central portion of the flap for measurement and analysis. Zernike coefficients $Z_{2}^{0}$ (defocus term) and $Z_{2}^{2} / Z_{2}^{-2}$ (astigmatism at $45^{\circ} / 90^{\circ}$ terms) were analyzed. The defocus term was used to calculate the surface area of the flap as a function of pressure:

$$
A_{\text {flap }}(p)=\int_{0}^{V_{\text {flap }}} Z_{2}^{0}(p) \cdot \sqrt[2]{3} \cdot\left(2 r^{2}-1\right) \mathrm{d} r
$$

where $A_{\text {flap }}$ is the surface area, $\mathrm{p}$ is pressure in Chamber 1 and $\mathrm{r}$ the distance from the center of the optical axis. Then, an analytical model was applied to obtain stress

$$
\sigma=p_{\text {chamber } 1} \cdot \frac{A_{\text {flap }}}{t h \cdot 2 r_{\text {flap }}}
$$

and strain

$$
\epsilon=\sqrt[2]{\frac{\Delta A_{\text {flap }}}{A_{\text {flap } 0}}}
$$


from changes in the flap area, where th stands for thickness, $\sigma$ for stress and $\epsilon$ for strain. Stress is a measure of the amount of force acting on the cross-sectional area, and strain represents the relative expansion of the original flap area. The absolute pressure variation, and hence the applied force stretching the flap, was sufficiently small, so that elastic deformation only could be assumed. A linear fit was adjusted to the stressstrain relation in order to obtain the corresponding Young's modulus:

$$
E=\frac{\Delta \sigma}{\Delta \epsilon}
$$

The Zernike terms $Z_{2}^{2}$ and $Z_{2}^{-2}$ were used in order to calculate $J_{0}$ and $J_{45}$ following the power vector notation. The calculated astigmatism was analyzed as a function of pressure increase. Absolute differences in the astigmatism between CXL and non-treated flaps were investigated. A students t-test was applied to test the statistical differences in the flap shapes across conditions: before and after in-situ CXL (paired, twotailed), un-treated and in vivo CXL (two sample equal variance, two tailed).

\section{Simple Inflation Model}

A simple analytic model was applied to estimate the corneal stressstrain relationship in whole globe inflation experiments (based on IOP variation). Changes in corneal thickness and radius of curvature were used to calculate the Young's modulus. The model was similar to that used in previous inflation tests, $[86,88,101,187]$ though fewer assumptions were required because the corneal radius of curvature was measured directly in the experiment (and not necessarily derived from the apical rise, which was the only experimental input data available in other studies[5, 56]). The model assumes that corneal thickness and mean radius of curvature are a function of IOP, as increasing IOP will make the globe of the eye expand. Corneal thickness decreases because the cornea becomes stretched over a larger area. However, stretching the cornea induces stress that acts to prevent further corneal extension. Both 
forces - the IOP tendency to stretch the cornea and the stress tendency to prevent it - arrange to form a stable equilibrium. With basic equations for pressure $p=\frac{F}{A}$ (where $\mathrm{p}$ represents pressure, $\mathrm{F}$ represents force, and A represents surface of eye globe) and stress $\sigma=\frac{F}{A}$ (where $\sigma$ represents stress, F represents force, and A represents corneal cross-sectional area), we can express stress as a function of IOP (see equation 1.41).

Limitations of the model include the fact that the mechanical properties of the sclera are not considered to contribute to the response of the cornea to pressure elevation because of the high rigidity and low elasticity of the sclera compared with the cornea.[7, 88] Of course, this is an approximation because scleral effects cannot be totally neglected. Nevertheless, it appears reasonable as a first estimation of stress-strain curves in whole eye globe experiments. Moreover, the stress-strain calculation refers to a homogenous membrane sphere, with the same elasticity, rigidity, and thickness all over the globe. Therefore, the influence of corneal thickness gradient or local elasticity and rigidity differences cannot be detected. In addition, the cornea is assumed to be purely elastic. The model is based on horizontal/vertical averages of corneal radii changes and does not include potential meridional differences in deformation.

\section{Corneal Stiffening Index}

For a better interpretation of different CXL treatment conditions, the corneal stiffening index (CSI) was introduced.

The longitudinal modulus of a material can be expressed in terms of its standard Young's modulus E' via the Poisson's ratio $\sigma$, i.e. $M^{\prime}=$ $E^{\prime}(1-\sigma) /(1+\sigma)(1-2 \sigma)$ In Brillouin measurements, the modulus M' is probed in a hypersonic frequency range of 5-10 GHz. It has been shown empirically that the Brillouin-measured longitudinal modulus M' is related to the conventional Young's (or shear) moduli E' through a $\log$-log linear relationship: $\log \left(M^{\prime}\right)=a \log \left(E^{\prime}\right)+b$, where a and $\mathrm{b}$ are material-dependent coefficients[175]. From the log-log relationship, the change of elastic modulus induced by a CXL protocol can be written as: 
$\frac{\Delta M_{2}^{\prime}}{M_{1}^{\prime}}=\alpha \frac{\Delta E_{2}^{\prime}}{M_{1}^{\prime}}$ From this relationship, it follows that the relative change of Brillouin modulus of two different procedures, $\Delta M_{2} / \Delta M_{1}$, is equal to the relative change of Young's modulus, $\Delta E_{2} / \Delta E_{1}$ : i.e.,

$$
\frac{\triangle M_{2}^{\prime}}{M_{1}^{\prime}}=\frac{\triangle E_{2}^{\prime}}{E_{1}^{\prime}}
$$

the Corneal Stiffening Index (CSI) is defined as a quantitative measure of the mechanical outcome of a specific CXL procedure (denoted by a subscript X) in direct comparison to the traditional Dresden protocol:

$$
C S I_{X} \equiv 100 * \frac{\Delta M_{X}^{\prime}}{\Delta M_{\text {Dresden }}^{\prime}} \equiv 100 * \frac{\Delta E_{X}^{\prime}}{\Delta E_{\text {Dresden }}^{\prime}}
$$

The CSI therefore compares the increase in corneal stiffness with respect to the standard CXL method. By definition, the CSI of the Dresden protocol is 100 . Any procedures that resulted in a smaller modulus change would have CSI less than 100. For example, CSI is 50 for a procedure that increases elastic modulus half as much as the Dresden method. Interestingly, CSI allows universal comparison between mechanical tests that use very different methods to assess mechanical properties, e.g. Brillouin microscopy, stress-strain tests, and shear rheometry.

\section{Theoretical Methods}

\section{Software}

\section{Matlab}

Matlab (The MathWorks, Natick, MA) was used to write custom routines for experimental and simulated data analysis as well as to calculate the merit function during optimization. 
Chapter 2. Methods

\section{ANSYS APDL}

ANSYS APDL 14.0 (ANSYS, Inc., Canonsburg, PA) was used for finite element simulation and optimization.

\section{ZEMAX}

ZEMAX 12 (Radiant Zemax Corporate, Redmond, WA), a commercial optical and illumination design software, was used to analyze the deformed geometry obtained from finite element simulations.

\section{Refractive Analysis of FE-models}

For the FE-simulation of intrastromal ring segment implantation, it was of special interest to study the refractive outcome after surgery.

\section{Corneal Refraction}

Two different methods were used to estimate the change in corneal refraction: (1) changes in the anterior corneal surface only (such as those measured by keratometry), and (2) changes in the refraction resulting from both the anterior and posterior corneal surface (such as those measured by refracting the eye).

ANTERIOR REFRACTION For (1) the mean radius of corneal curvature in a central region of 4-mm diameter was estimated by fitting a circle to the coordinates of the anterior surface (obtained from the FE model). The keratometer equations were applied to determine the mean corneal refraction, assuming a refractive index of $n=1.3375$ (as done in many commercial keratometers). Calculations were performed using Matlab.

Overall REFraction For (2), the change in corneal power (pre- and postsimulated implantation) was computed by ray tracing through the anterior and posterior corneal surface (coordinates obtained from the FEM analysis and fitted using extended cubic splines). The distance between 
both surfaces was set by the corneal thickness at the apex. Refractive indices of $n_{\text {Cornea }}=1.376, n_{\text {Water }}=1.336$, and $n_{\text {Vacuum }}=1.000$ were assumed for the cornea, aqueous humor, and air, respectively. Calculations were performed using ZEMAX. Corneal power was estimated for different pupil diameters, ranging from 3- to 6-mm diameters. These calculations included both the contributions of corneal shape and potential changes in the displacement of the corneal apex. Changes in refraction were calculated considering that the retina does not move (i.e., an axial shift of the apex reduces the focal length of the eye).

\section{Spherical Aberration}

Changes in the fourth-order Zernike spherical aberration were calculated using ray tracing. Aberrations were evaluated in the pupil plane at a distance of $3 \mathrm{~mm}$ from the cornea. The spherical aberration of the anterior corneal surface was estimated assuming a single surface (defined by the coordinates of the simulated cornea, fitted by cubic splines) and the corneal index of refraction $(n=1.376)$. The spherical aberration of the entire cornea was estimated on a two-surface model (implemented in Zemax as described above).

\section{Finite Element Model}

For finite element simulations of the corneal tissue, a two-dimensional axis-symmetric geometry was used. As the objective of this dissertation is to address the static and dynamic material properties, we decreased complexity regarding the geometry, but evaluated material nonlinearities and time dependency instead.

\section{Air-puff FEM}

In chapter 9 a finite element model is presented to simulate the corneal response to an air-puff. Transient structural analysis was performed and the temporal and spatial geometrical deformations were studied. 
Chapter 2. Methods

\section{Vibrography FEM}

In chapter 10 a finite element model is presented to study the corneal resonance behavior under vibration. Modal analysis was performed in order to extract the natural frequencies and mode shapes.

\section{ICRS FEM}

In chapter 11 a finite element model is presented to predict the refractive outcome after intrastromal ring segment implantation. Static structural analysis was performed in order to retrieve the long-term deformations of the corneal geometry.

\section{FEM Solver}

A sparse solver (included in the ANSYS code) was applied in order to directly solve equation 1.26 . For modal analysis the full solution method chosen.

\section{Sensitivity Analysis}

A sensitivity analysis was performed for the best geometry and material parameters in order to determine the impact of the different mechanical parameters on the merit function. For this purpose different geometrical and mechanical input parameters were changed successively (with respect to one reference parameter set) by $+20 \%$. Then the finite element model was solved for the new parameter set, and the deformed nodes were exported to Matlab, where the geometrical changes were analyzed and the differences to the reference set determined. The sensitivity analysis was used in order to find the dominant parameters for the observed corneal response. 


\section{3}

\section{Biomechanical Changes after}

Collagen Cross-Linking from

\section{Porcine Eye Inflation Experiments}

Given that the increase in corneal rigidity afte UV cross-linking only had been quantified with strip extensiometry tests [226], we developed a procedure to record the corneal stres-strain curve from inflation experiments of whole eye globes in order to maintain the natural geometry and hence natural bidirectional stress distribution.

This chapter is based on the publication by Sabine Kling et al, entitled "Corneal biomechanical changes after collagen cross-linking from porcine eye inflation experiments" in Investigative Ophthalmology and Vision Science. The co-authors of this work are Laura Remon, Alfonso 
Chapter 3. Biomechanical Changes after Collagen Cross-Linking from Porcine Eye Inflation Experiments

Perez-Escudero, Jesus Merayo and Susana Marcos. This work was also presented in a poster contribution at the 2009 Annual Meeting of the Association for Research in Vision and Ophthalmology (ARVO) in Fort Lauderdale (Florida, USA) and in an invited oral contribution at the 2010 ASETCIRC (Asociación Española de Tecnología y Cirugía de Implantes, Refractiva y Córnea) meeting in Madrid (Spain).

The author of this thesis (i) did the sample preparation, (ii) performed the measurements in collaboration with L. Remon, (iii) processed the data and analyzed the results and (iv) prepared the manuscript in collaboration with S. Marcos. 


\section{Introduction}

Corneal collagen cross-linking [CXL] is an emerging technique aimed at strengthening corneal tissue. It has been successful in slowing down the progression of keratoconus and preventing ectasia. The technique works through the application of riboflavin (vitamin B2) as a photosensitizer and irradiation of the cornea with UV-A to increase the formation of intrafibrillar and interfibrillar covalent bonds by photosensitized oxidation (explained in more detail in 1). After it was first proposed by Seiler[195] in 1998, several studies have examined corneal CXL with regard to dosage parameters of riboflavin, safety of UV-A radiation, [196, 226, 227] penetration depth,[222] and efficacy of the treatment in keratoconus[221] or of the combined effect of corneal surgical treatments (i.e., intracorneal rings, laser refractive surgery, or orthokeratoplasty).[60, 79] The formation of additional cross-links between individual collagen molecules has not been verified yet, however its direct effect - an increase in corneal rigidity.

Biomechanical changes have been demonstrated in human,[113, 221] porcine[113] and rabbit[198, 224] corneas. To a large extent, these studies rely on static stress-strain tests on corneal strips. A recent study reported a significant increase in corneal rigidity after cross-linking, indicated by a rise in stress in treated porcine corneas (by 71.9\%) and human corneas (by 328.9\%) and in Young's modulus by a factor of 1.8 in porcine corneas and of 4.5 in human corneas[226] using extensiometry methods. Previous works have stressed the limitations of corneal strip extensiometry measurements to measure biomechanical properties. [56] Some limitations include the fact that strip specimens originate from a curved sample, the disruption of the corneal structure because the lamellae are cut, and that several crucial constraints are ignored (real pachymetry, or meridional differences, among others). Although some models have been developed to improve the accuracy of extensiometry tests, [56] it has been suggested that the resulting material stress-strain 
Chapter 3. Biomechanical Changes after Collagen Cross-Linking from Porcine Eye Inflation Experiments

relationship from strip extensiometry is considerably stiffer than that derived using inflation tests. The differences between the reported estimates of Young's Modulus using different techniques vary by two orders of magnitude.

Whole-eye globe inflation testing overcomes many of the problems of extensiometry, although care must be taken to control tissue hydration if the results aim to mimic in vivo biomechanical response. Inflation testing methods have been used for many years to derive ocular rigidity[7, 101, 151] and to determine the relationship between intraocular pressure and corneal apex displacement $[5,56,101]$. Some studies $[5,16]$ use only the anterior portion of the globe (cornea and a ring of sclera) attached to a chamber with a complex fixture scheme to separate corneal deformation from scleral deformation. Although this technique has the advantage of avoiding the potential influence of the supporting sclera in the measurements, the use of the intact eye represents a closer situation of the in vivo response. Also the mechanical fixation of the sclera induces a non-physiological boundary condition that could affect the strain distribution along the cornea. Given that the Young's modulus of the sclera is 3 to 3.5 times higher than the cornea,[7] the impact of the scleral biomechanical properties on the corneal deformation measurements is potentially small. In the whole eye model, the limbal junction is allowed to move, which is thought to be crucial to establish a biomechanical model mimicking the optical function of a real eye.[101, 187, 200, 229]

To our knowledge, this study presents for the first time inflation tests on porcine corneas that had undergone a collagen cross-linking treatment, in comparison to non-crosslinked corneas. Also, while most inflation experiments only report the shift of the corneal apex in response to the variation of intraocular pressure, we report also measurements of anterior and posterior corneal shape and thickness as a function of increased and decreased pressure. These results allow us to accomplish direct comparisons with clinical refractive changes measured in vivo. In addition, these measurements can be used to derive estimates of 
stress-strain behavior and Young's modulus after cross-linking (and to estimate the changes of corneal stiffness and biomechanical properties induced by the treatment). The full parametric characterization of corneal deformation as a function of pressure will be very valuable to improve predictability of finite element modelling of the cornea and ultimately the predictability of the procedure.

\section{Protocols}

A total of 38 fresh enucleated porcine eyes (see section 2) were used in the study. 23 eyes were treated with standard UV cross-linking (see chapter 2). Control eyes $(n=15$, contralateral eyes from the treated eye) were not irradiated with UV light, but followed epithelium removal and riboflavin instillation. 16 cross-linked eyes and 8 control eyes were measured under varying intraocular pressure, and 7 cross-linked and 7 control eyes were measured under constant pressure. Measurements were performed within 4 hours post-mortem (immediately after treatment) and then repeated 24 hours afterwards. Between measurements the eyes were kept in a chamber with cotton soaked in physiologic saline (sodium chloride $0.9 \%$ ) in a refrigerator at $4{ }^{\circ} \mathrm{C}$. The enucleated porcine eyes were placed in a custom-made whole-eye-globe holder (described in chapter 2 ) to guarantee proper centration during the application of the treatment and during the measurements. The eyes always were placed with the longer side of the cornea in the vertical direction. Compared with the real orientation of the eye in the pig, in the experiment the eye was turned $90^{\circ}$, which allowed a better view and more accurate alignment. (In Results and Discussion, however, we use horizontal and vertical to refer to the actual orientation of the eye in the pig.) The eye holder was placed in a wet-chamber (see chapter 2). A needle was introduced through the optic nerve head up to the vitreous and connected the eye with an infusion tube filled with $\mathrm{NaCl}$ solution. The infusion tube then was automatically driven to adjust the IOP (more details in chapter 2). Preliminary measurements 
Chapter 3. Biomechanical Changes after Collagen Cross-Linking from Porcine Eye Inflation Experiments

were conducted by entering the anterior segment of the eye superiorly, through the sclera, leaving the cornea untouched (Pérez-Escudero A, et al. IOVS 2008;49:ARVO E-Abstract 664) However,this procedure was more challenging, and we did not observe any differences between results. Pentacam measurements were taken at IOP steps of $4 \mathrm{mmHg}$ increase (or decrease) as controlled by the pressure monitored system or every 5 $\mathrm{min}$ (in the experiments were the IOP was kept constant at $19 \mathrm{mmHg}$ ). Temperature and humidity were recorded every 2 seconds throughout the experiment. An entire experimental session on each eye took typically 80 minutes. The initial measurement was taken at $18.5 \mathrm{mmHg}$, and then subsequently after $4 \mathrm{mmHg}$ steps increase. The pressure was increased up to $58.5 \mathrm{mmHg}$, and then decreased, at $4 \mathrm{mmHg}$ steps, with data also collected sequentially. Sessions on both eyes of the same animal were typically performed consecutively, and randomly, with one eye having undergone the cross-linking treatment and the other one only epithelial removal and riboflavin instillation. A set of preliminary measurements was performed increasing simultaneously the pressure in both eyes, and taking consecutive images in each eye at identical pressure levels. We did not observe any difference between the results from this procedure, and those from automatic full measurements in one eye after the other. A set of control experiments was performed by keeping the pressure constant at $19 \mathrm{mmHg}$, and taking images at 5-min intervals during the same period of time than the variable-pressure experiments. Measurements were also collected in cross-linked and not-cross-linked eyes. Typically each eye was measured in two identical experimental sessions, 24 hours apart.

\section{Set-up}

The measurement setup to image the corneal deformation during IOP variation is illustrated in Figure 2.9. The set-up is composed of a Scheimpflug camera (see chapter 1) and an automatically controlled pressure pump (see chapter 2). The water column was filled with $0.9 \%$ saline solution and connected to the eye globes to the pressure pump in 
order to change the IOP. The eyes were measured within a wet chamber (see chapter 2, where temperature and humidity were continuously monitored.

\section{Data analysis}

Corneal thickness and mean corneal curvature were obtained for all conditions and IOP levels (in a central zone of $6 \mathrm{~mm}$ ). The mean corneal thickness was directly taken from the Scheimpflug imaging commercial software. The apical mean radii of curvature were obtained from conic fittings of the anterior and posterior corneal elevation data. As changes in corneal thickness changes are more directly related to the corneal biomechanical properties (the curvature changes also depend largely on the overall geometry and scleral properties), we set our main focus on corneal thickness changes happening under 3D stress-strain testing. Measurements of horizontal and vertical radii of curvature and asphericity were obtained by fitting the raw elevation maps provided by the software to biconic surfaces, using custom routines written in Matlab (Mathworks, MA, USA) [25, 154]. Central pachymetry was obtained directly from the commercial software. The axial shift of the corneal apex was estimated by measuring horizontal and diagonal distance from the apex to a constant reference point in Pentacam Scheimpflug images (white dot in the upper left corner). These data were used to calculate the apical displacement on a subset of eyes (4 untreated eyes and 4 cross-linked eyes), which were not moved at all during the measurements. Three-dimensional image acquisition with this system (25 meridional sections per image) was performed automatically and in synchronization with an inflation system, as described in a previous publication[109]. 
Chapter 3. Biomechanical Changes after Collagen Cross-Linking from Porcine Eye Inflation Experiments

\section{Results}

\section{Changes in corneal thickness}

A dramatic decrease in corneal thickness occurred immediately after the application of riboflavin-dextran solution (during 30 minutes) to the de-epithelizated corneas. Figures 3.1 and 3.2 shows examples of Scheimpflug images before and after riboflavin application. On average a decrease of $281 \pm 5 \mu \mathrm{m}$ was found after application of riboflavin. A further decrease was observed after UV-illumination which was higher in control eyes $(88 \mu \mathrm{m})$ than in cross-linked eyes $(31 \mu \mathrm{m})$. After riboflavin instillation the radius of curvature changed by $165 \pm 655 \mu \mathrm{m}$ (anterior) and $-774 \pm 1689 \mu \mathrm{m}$ (posterior).

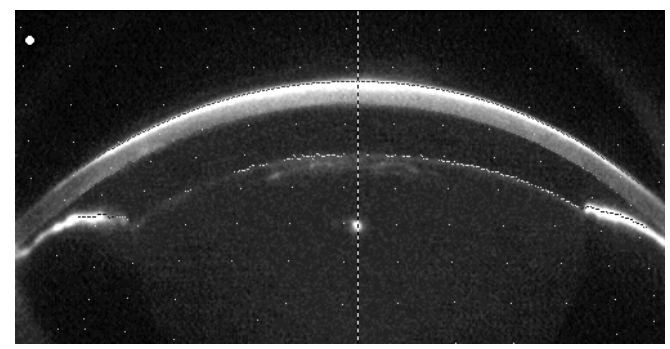

Figure 3.1: Before Riboflavin-Dextran instillation

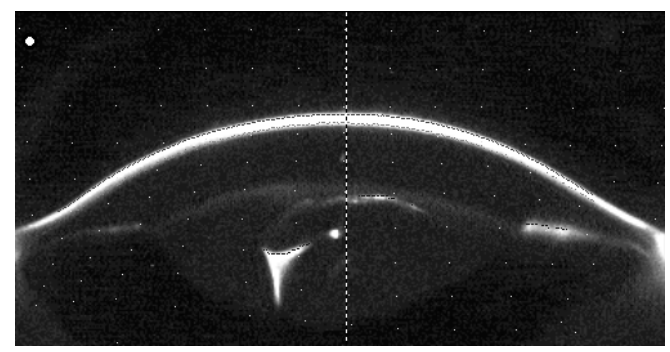

Figure 3.2: 30 min after Riboflavin-Dextran instillation

Figure 3.3 shows the change in corneal thickness as a function of IOP change (first increased from $18.5 \mathrm{mmHg}$ to $58.5 \mathrm{mmHg}$, then decreased), 
immediately after the treatment (A) and 24-hour after treatment (B). Results are the average across 16 cross-linked eyes and 8 non- crosslinked eyes. In each case the change is referred to the initial corneal thickness in each eye. Initial corneal thickness varied between 430$636 \mu \mathrm{m}$ in the cross-liked eyes and 353- $647 \mu \mathrm{m}$ in the non-cross-linked eyes immediately after the treatment (A) and between $999-1221 \mu \mathrm{m}$ in the cross-liked eyes and $945-1259 \mu \mathrm{m}$ in the non-cross-linked eyes 24 hours after the treatment (B). Immediately after the treatment corneal thickness (Figure 3.3A) changes are dominated by hydration effects, as the increase in corneal thickness occurs similarly under variable IOP $(1.03 \mu \mathrm{m} / \mathrm{min}$ (cxl) and $0.83 \mu \mathrm{m} / \mathrm{min}$ (untreated)) or constant IOP $(1.1 \mu \mathrm{m} / \mathrm{min}$ (cxl) and $2.0 \mu \mathrm{m} / \mathrm{min}$ (untreated)). Removal of the epithelium made the corneas more susceptible to hydration, as under humidity and pressure controlled conditions in the same moist chamber we had not observed an increase of corneal thickness at constant IOP in virgin corneas in previous experiments in our laboratory.

After 24 hours hydration effects are minor (around 10\% of the values immediately after the treatment, as shown from data under constant IOP), and are dominated by the pressure change. Corneal thickness clearly decreased with IOP increase, and increased with IOP decrease, with smaller changes in the cross-linked than in the non-cross-linked corneas. A total variation of $40 \mathrm{mmHg}$ in IOP pressure produced a decrease in corneal thickness of $51.2 \mu \mathrm{m}$ (in cross-linked corneas) and $76.3 \mu \mathrm{m}$ (in non- cross-linked corneas). On average, the changes in non-cross-linked eyes were significantly higher (by 1.54) than in cross-linked eyes. The differences were statistically significant at all pressure levels $(\mathrm{p}<0.01)$. After IOP variation cross-linked corneas returned to their initial thickness whereas non-cross-linked corneas remained $42.1 \mu \mathrm{m}$ thinner.

\section{Changes in the corneal apex position}

Figure 3.4 shows the change in corneal shape position as a function of pressure for cross-linked and non-crossed linked eyes. The apical rise of 
Chapter 3. Biomechanical Changes after Collagen Cross-Linking from Porcine Eye Inflation Experiments

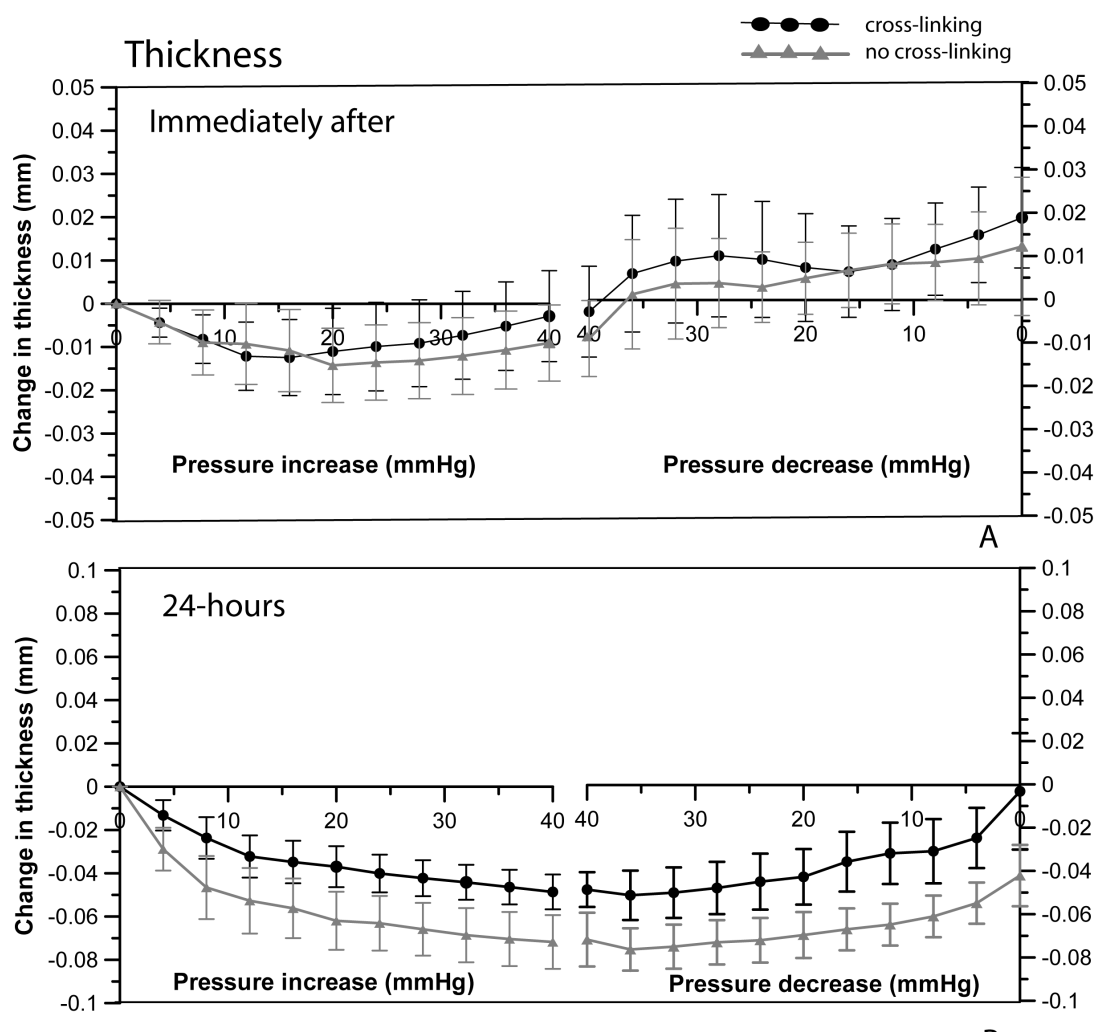

Figure 3.3: Change in corneal thickness with IOP pressure, for crosslinked corneas $(\bullet)$ and non cross-linked corneas ( $\Delta$ ). Symbols are average data across eyes and the error bars stand for standard deviatons. A. Immediately after the treatment. B. 24-hours after the treatment. Data are average of $16 \mathrm{cxl}$ and 8 non-cxl. Error bars represent standard deviation. 

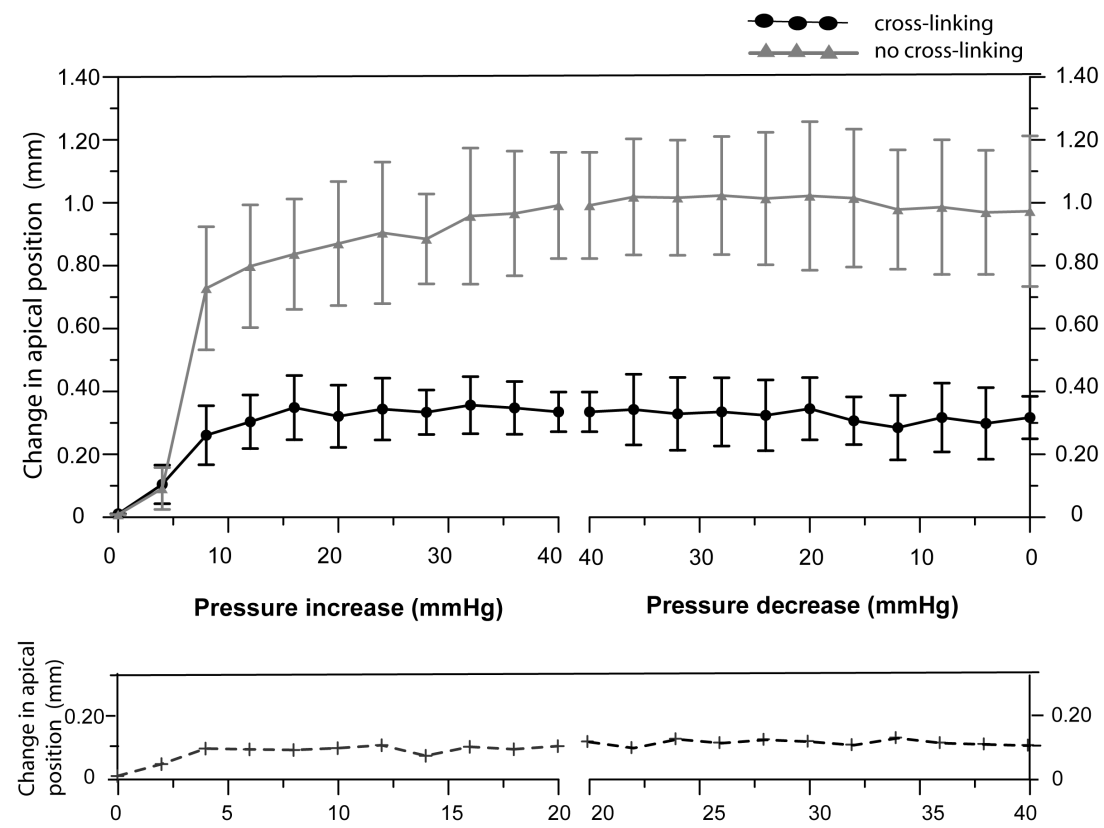

Time (min.)

Figure 3.4: Apex shift as a function of (increasing and decreasing) pressure. Cross-linked corneas are represented by $\bullet$ and non-cross-linked by $\Delta$. Apex shift during 40 minutes (equivalent to duration of the pressure variation experiment) under constant pressure is shown in dashed line. Data are immediately after treatment. Data are average of $4 \mathrm{cxl}$ and 4 non-cxl. Error bars represent standard deviation. 
Chapter 3. Biomechanical Changes after Collagen Cross-Linking from Porcine Eye Inflation Experiments

control eyes that had not undergone pressure changes during the same period of time is also shown for reference. There was a significant forward shift in the anterior corneal apex with increasing pressure, which was 2.8 times higher in non-treated corneas $(962 \mu \mathrm{m})$ than in cross-linked corneas $(345 \mu \mathrm{m})$. With constant pressure the apex only showed a slight shift and no difference between treated and untreated corneas. Decreasing pressure produced a backward movement of the apex, but it did not return to the initial values.

\section{Change in horizontal and vertical corneal radius of cur- vature}

Immediately after the procedure we found slight variation in the anterior corneal curvature throughout the session under constant IOP: -4.5 $\mu \mathrm{m} / \mathrm{min}$ (cxl), $4.4 \mu \mathrm{m} / \mathrm{min}$ (non-cxl). The variation was even smaller under constant IOP 24 hours after treatment: $-0.63 \mu \mathrm{m} / \mathrm{min}$ (cxl), - 2.5 $\mu \mathrm{m} / \mathrm{min}$ (untreated). Figures 3.5 and 3.6 show changes of the anterior (Figure 3.5) and posterior (Figure 3.6) corneal radius of curvature in the horizontal meridian with IOP variation, (A) immediately after treatment (B) and 24-hours after the treatment.

Changes in the anterior corneal radius with pressure, as well as the effect of treatment, are minimal immediately after the treatment. Measurements 24 hours after treatment (Figure 3.5 A and 3.6B) showed statistically significant differences between cross-linked and non-cross-linked eyes. The radius of curvature decreased with IOP increase in cross-linked eyes, very similarly in both the anterior and posterior corneal surface $(-309 \mu \mathrm{m}$ (anterior), $-319 \mu \mathrm{m}$ (posterior) at $40 \mathrm{mmHg} \mathrm{IOP}$ ) whereas control eyes showed an increase in radius of curvature with IOP increase (anterior by $221 \mu \mathrm{m}$ ) or remained constant (posterior). With decreasing IOP, the anterior cornea nearly returned to its initial curvature $(-113 \mu \mathrm{m}$ (cxl), 39.6 $\mu \mathrm{m}$ (non- crosslinked)) whereas the posterior cornea remained practically constantly flattened (-180 $\mu \mathrm{m}$ (cxl), $-220 \mu \mathrm{m}$ (untreated)).

Changes in the vertical corneal radius with IOP (Figure 3.7 for anterior

90 de 329 

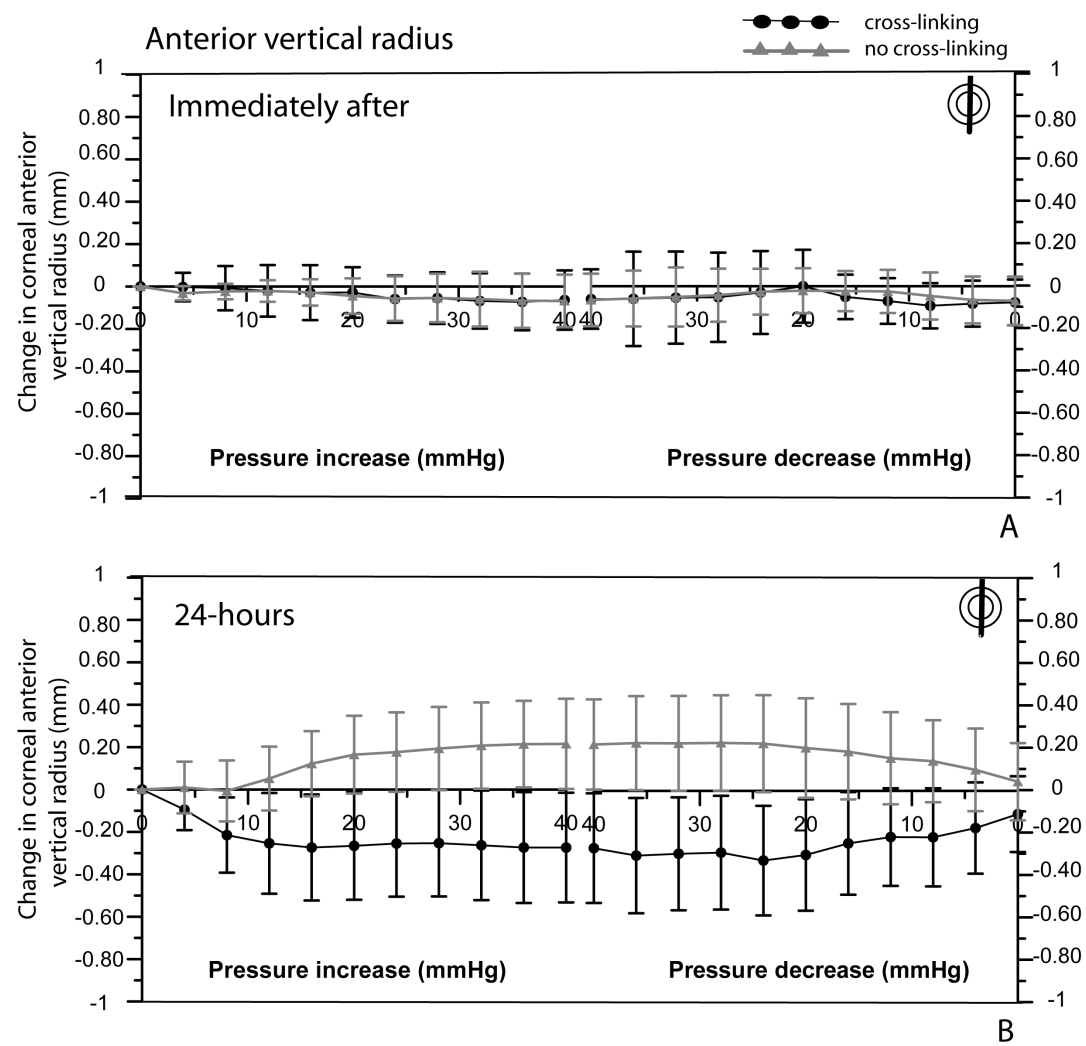

Figure 3.5: Change in anterior horizontal radius of curvature with varying pressure, immediately after $(A)$ and 24-hours after treatment $(B)$. Cross-linked corneas are represented by $\bullet$ and non cross-linked corneas by $\Delta$. Data are average of $16 \mathrm{cxl}$ and 8 non-cxl. Error bars represent standard deviation 
Chapter 3. Biomechanical Changes after Collagen Cross-Linking from Porcine Eye Inflation Experiments
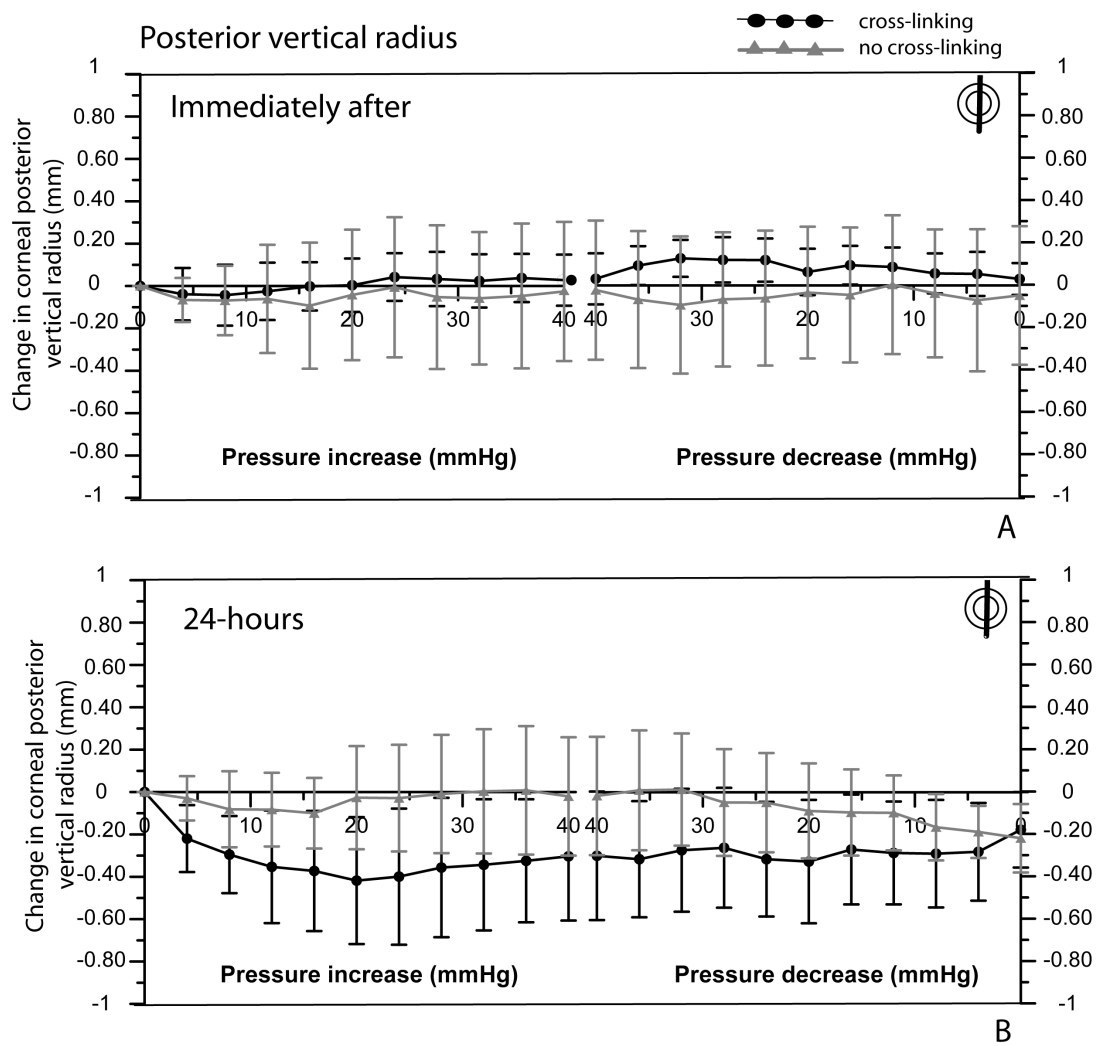

Figure 3.6: Change in posterior horizontal radius of curvature with varying pressure, immediately after (A) and 24-hours after treatment $(B)$. Cross-linked corneas are represented by $\bullet$ and non cross-linked corneas by $\Delta$. Data are average of $16 \mathrm{cxl}$ and 8 non-cxl. Error bars represent standard deviation. 

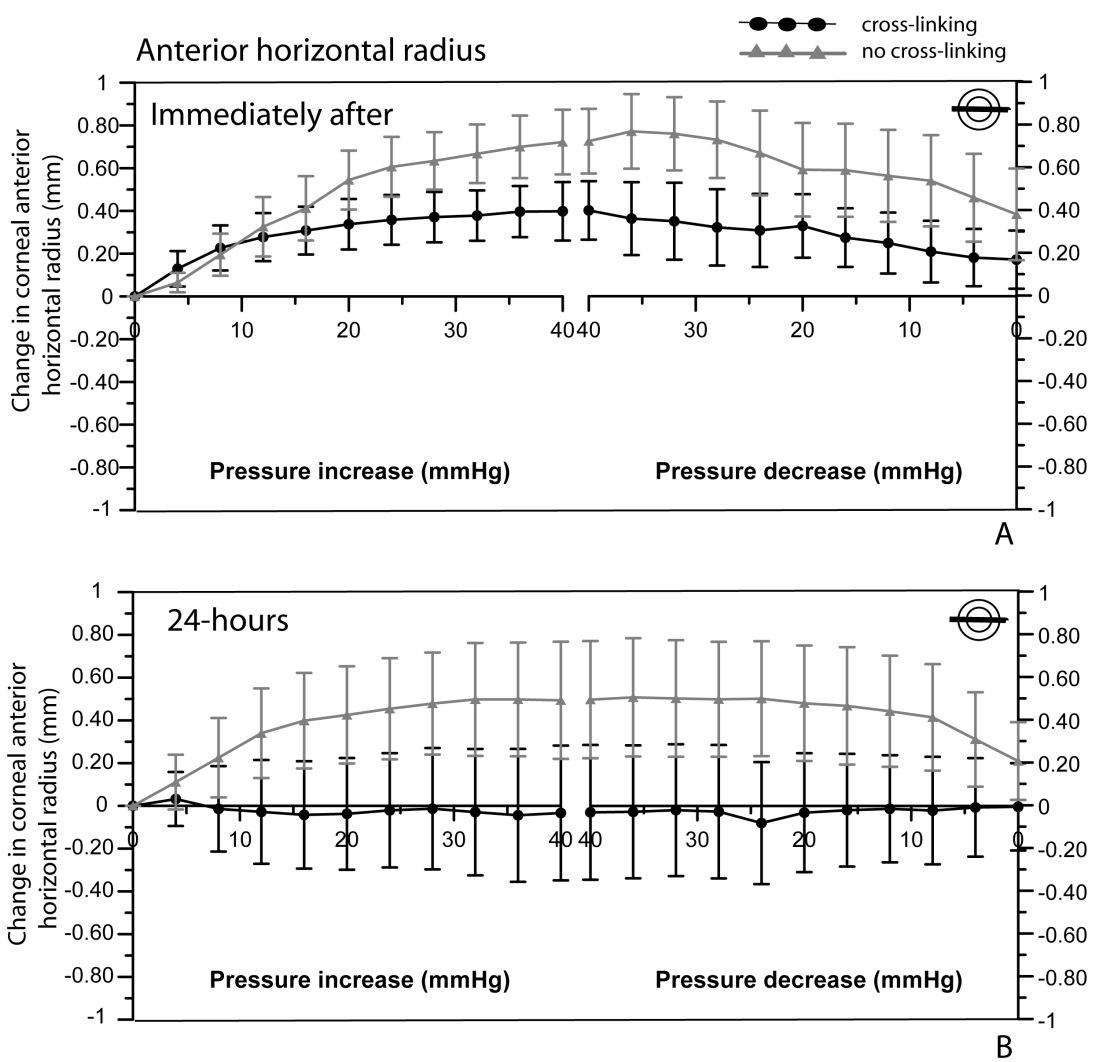

Figure 3.7: Change in anterior vertical radius of curvature with varying pressure, immediately after (A) and 24-hours after treatment (B). Cross-linked corneas are represented by - and non cross-linked corneas by $\mathbf{\Delta}$. Data are average of $16 \mathrm{cxl}$ and 8 non-cxl. Error bars represent standard deviation. 
Chapter 3. Biomechanical Changes after Collagen Cross-Linking from Porcine Eye Inflation Experiments

cornea and Figure 3.8 for posterior cornea), as well as differences between the cross-linked and non-cross-linked corneas, were more dramatic than for the horizontal radius. Figure 3.7 A and 3.8 A show the behavior immediately after treatment, and 3.7 B and 3.8 B 24 hours after treatment. In contrast to corneal thickness and horizontal radius curvature, immediate measurements of vertical radius curvature under IOP variation already showed larger changes in non-crossed-linked corneas $(768 \mu \mathrm{m}$ (anterior), $665 \mu \mathrm{m}$ (posterior) at $40 \mathrm{mmHg}$ IOP) than in cross-linked ones ( $398 \mu \mathrm{m}$ (anterior), $448 \mu \mathrm{m}$ (posterior) at $40 \mathrm{mmHg}$ IOP). Measurements 24 hours after treatment showed the same tendency. Non-cross-linked corneas deformed significantly more upon IOP variation than cross-linked corneas $(\mathrm{p}<0.01)$ Non-cross-linked corneas eyes showed a large increase in the vertical radius of curvature with IOP increase $(504 \mu \mathrm{m}$ (anterior), $679 \mu \mathrm{m}$ (posterior) at $40 \mathrm{mmHg}$ IOP) in comparison with cross-linked corneas $(248 \mu \mathrm{m}$ (anterior) and $0 \mu \mathrm{m}$ (posterior)), as well as a strong decrease with IOP decrease. Non-crosslinked corneas experienced a plastic deformation as they did not return to their original radii of curvature (remaining $207 \mu \mathrm{m}$ (anterior), $264 \mu \mathrm{m}$ (posterior) flatter) after IOP variation. Changes in radius of curvature of non-cross-linked eyes are x5.9 higher in vertical than in horizontal meridian whereas changes of cross-linked eyes are $\mathrm{x} 4.3$ times in horizontal than in vertical meridian. Control experiments showed that the changes in anterior and posterior vertical radius of curvature were minimal under constant pressure throughout a session of the same duration. Changes in radius under constant pressure ranged between $-4.4 \mu \mathrm{m} / \mathrm{min}$ in cross-linked corneas immediately after treatment to $0.057 \mu \mathrm{m} / \mathrm{min}$ in cross-linked corneas 24 hours after treatment.

\section{Refractive changes: varying versus constant pressure}

The analysis in previous subsections was based on changes in corneal radius of curvature relative to the value of the radius of curvature at the beginning of the session. However, we observed consistent changes in the radius of curvature between the two sessions separated by 24 

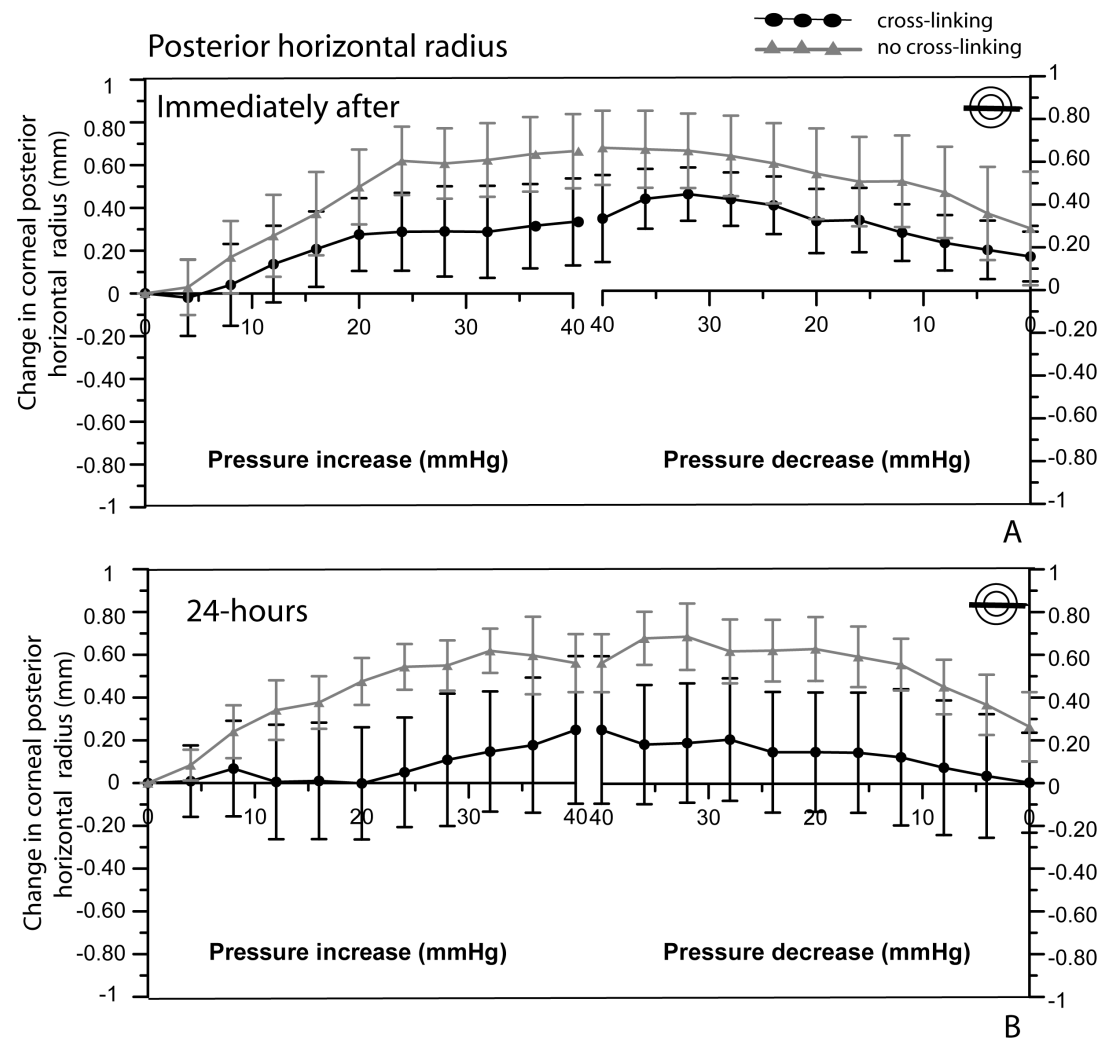

Figure 3.8: Change in posterior vertical radius of curvature with varying pressure, immediately after (A) and 24-hours after treatment $(B)$. Cross-linked corneas are represented by $\bullet$ and non cross-linked corneas by $\Delta$. Data are average of $16 \mathrm{cxl}$ and 8 non-cxl. Error bars represent standard deviation. 
Chapter 3. Biomechanical Changes after Collagen Cross-Linking from Porcine Eye Inflation Experiments
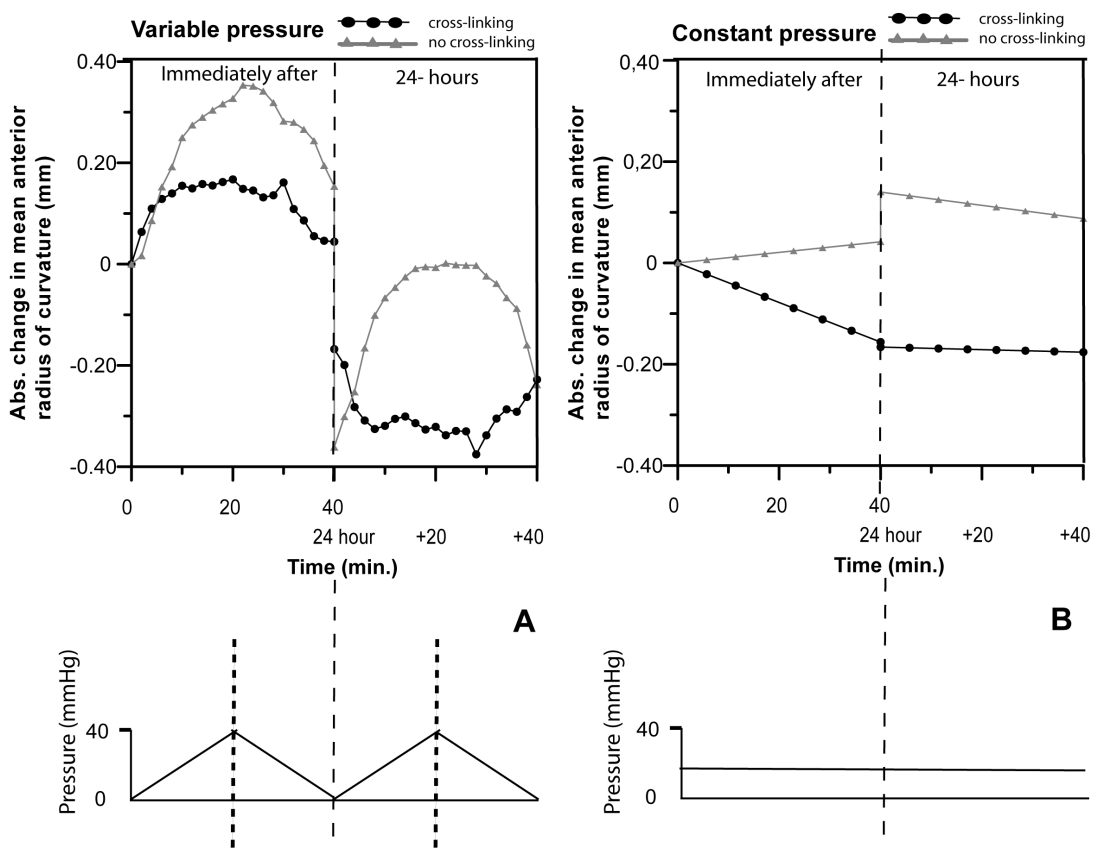

Figure 3.9: Change in anterior radius of curvature (averaged horizontal and vertical) as a function of time, relative to the value at the beginning of the experiment. A. For variable pressure. B For constant pressure. The accompanying graphs represent the change of pressure with time, Cross-linked eyes are shown with $\bullet$ and non-cross-linked eyes with ^. Pressure variations induced a characteristic corneal deformation (much larger in untreated than cross-linked corneas). Cross-linking produced also a significant decrease in corneal curvature (even under constant pressure). 
hours. Figure 3.9 shows the variation in anterior radius of curvature (arithmetic mean of horizontal and vertical radii), averaged across eyes, relative to the initial value of the experiment on day 1 in each eye. Figure 3.9 A shows data with varying IOP (both immediately after and 24 hours after treatment). Figure 3.9 B shows the data under constant IOP. Both conditions reveal that the cross-linking treatment produces a decrease in the corneal anterior radius of curvature (of $156 \mu \mathrm{m}$, from the data under constant IOP). This corneal steepening occurred immediately after treatment (at least during the $40 \mathrm{~min}$ of observation) and then remained constant (also overnight). Under variable pressure, although the measurements in both sessions (immediately and 24 hour after) show a similar pattern (large variations with IOP in non-cross-linked eyes and smaller variations in cross-linked eyes), there is a steepening from day 0 to day 1 .

\section{Simple Biomechanical Model: Young's Modulus and Hys- teresis}

Figure 3.10 plots stress as a function of strain $(\sigma(\epsilon))$ obtained using the theoretical computations for untreated and crosslinked eyes described (Figures 3.10A [immediately after treatment], 3.10B [after 24 hours]). Behavior with both the increasing and the decreasing pressure is represented. The slope of these curves in the linear region (first phase), for increased pressure, represented a measure of the Young's modulus of the cornea - the steeper the curve, the stiffer the cornea. The increase in stiffness was clear immediately after the cross-link treatment. In addition, although there were some differences between the immediate and the 24-hour behavior, the main qualitative features remained. The major change after 24 hours occurred in the untreated cornea, most likely because of the effects of hydration. After 24 hours, the Young modulus in the crosslinked eyes $(1.096 \pm 0.30 \mathrm{MPa})$ was significantly $(1.58 \mathrm{x})$ higher than that of untreated eyes $(0.692 \pm 0.30 \mathrm{MPa})$; therefore, cross-linking stiffened corneal tissue by $36.86 \%$. The model also allowed us to eval- 
Chapter 3. Biomechanical Changes after Collagen Cross-Linking from Porcine Eye Inflation Experiments
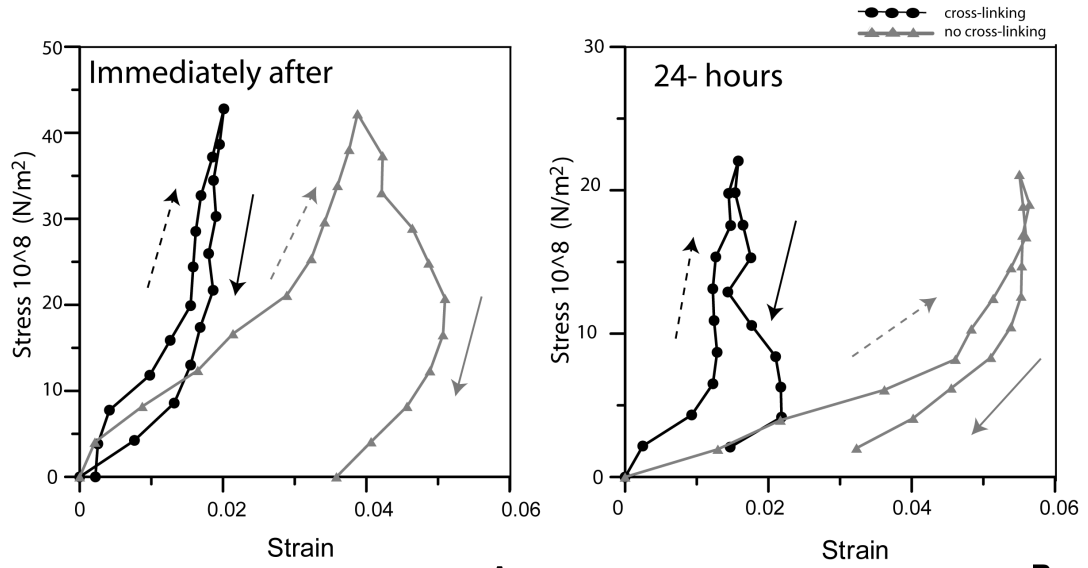

A

B

Figure 3.10: Strain-stress curves derived from equations 1.41 and 2.3. - are for cross-linked corneas and $\Delta$ for untreated corneas. The left part of the curve corresponds to increased pressure and the right part of the curve to the decreased pressure. A. Immediately after treatment. $B$. After 24-hours.

uate the differences in the strain on increased or decreased stress. We calculated the area inside the curves presented in Figure 3.8. Although not directly a measure of hysteresis, this measure relates to the elastic behavior of the cornea. We found that the ratio of untreated/cross-linked cornea stress-strain curve area was 12.8 immediately after treatment; 24 hours after treatment, it was 1.38 . Time (and probably differential effects of hydration) produced changes in opposite directions in the untreated and crosslinked corneas. We found that after decreasing pressure from $58 \mathrm{~mm} \mathrm{Hg}$ to the initial pressure, cross-linked corneas almost returned to their initial start points (strain, 0.002 [immediately] and 0.014 [24 hours later]), whereas control corneas showed a large hysteresis and ended at higher strain (0.036 [immediately] and 0.032 (24 hours later). We conclude that crosslinked corneas were more elastic (although stiffness is higher) and did not show any remaining plastic deformations, in contrast to control eyes. 


\section{Discussion}

\section{Comparison to previous clinical and experimental data}

The first major effect found during treatment is the decrease of corneal thickness after riboflavin instillation, which must be caused by the fact that riboflavin-dextran solution is hypertonic. We have observed that this has a strong dehydrating effect on the porcine cornea, which is reduced in thickness by $54 \%$. Decrease in corneal thickness in patients has been very recently reported in a clinical study,[121] in which corneal pachymetry was monitored using Pentacam Scheimpflug imaging immediately following riboflavin instillation. On average, the corneal thickness decreased by $18.7 \%$, which may pose some safety concerns in thin corneas. Alternative hypoosmolar riboflavin solution has been proposed to produce corneal swelling rather than dehydration to increase the effective corneal thickness[80]. We observe an increase in corneal thickness caused by swelling 24 hours after treatment, as reported in previous studies on porcine corneas. [222] However we did not find that corneal hydration interfered with the corneal response to pressure variation 24 hours after the treatment, neither that swelling effects were different between untreated or cross-linked cornea.

Even at constant pressure, we found a decrease in corneal radius of curvature 24 hours after cross-linking $(166 \mu \mathrm{m})$. This is consistent with clinical reports of corneal steepening during the first moth after treatment. [44] After the initial steepening, and six-month after treatment a decrease in corneal curvature (flattening) of $2 \mathrm{D}$ on average, and a decrease in refractive error of $1.14 \mathrm{D}$ [214] have been reported clinically. Our results show immediate effects of cross- linking, as well as 24 hours after treatment. The fact that refractive changes are observed in patients during the 6-months following the treatment suggests would healing or long-term acting balancing forces.

Previous inflation studies on excised porcine corneas showed a rise of the apex as a function of increased IOP very similar to our observations 
Chapter 3. Biomechanical Changes after Collagen Cross-Linking from Porcine Eye Inflation Experiments

in untreated corneas (Figure 3.4). As in Elsheikh and Anderson[56] we found an initial phase $(0-24 \mathrm{mmHg})$ where the corneal apex rises with pressure at a low stiffness (relatively high slope in Figure 3.4), attributed to a relative looseness of the collagen fibers, followed by a much increased stiffness in the second phase, where a significant increase in pressure results in a very small apical rise, attributed to the collagen fibers becoming taut. Previous studies used trephinate specimens (cornea and a narrow ring of scleral tissue) attached to a wet chamber as opposed to the whole globe used in the current study. The higher relative apical rise found in our study may result from height changes in the limbus, as opposed to the mechanical rigid attachment in the trephinate experiments. The limited lateral range of the Scheimpflug camera prevented us from accounting the peripheral forward shift. Crosslinking reduced apical rise by a factor of 2.8 with respect to untreated corneas. Most notably, in cross-linked corneas the second phase is reached at significantly lower loads $(15 \mathrm{mmHg})$, so that the regime at physiological IOP is dominated by collagen with a much higher stiffness.

Although we cannot rule out a contribution of the entire eye motion to the apical radius displacement, the behavior we found in corneal apical shift was consistent with the changes in corneal thickness. Non-crosslinked corneas did not fully return to the initial values after increased and decreased IOP with regard to apical position or corneal thickness. Crosslinked corneas tended to return to the original values both for apical position (within the measurement variability) and for corneal thickness. These results are consistent with the cross-linked cornea being stiffer (but with a more elastic behavior) than the non-cross-linked cornea.

A previous study[100] reported the decrease in central corneal thickness on globe inflation in two virgin human corneas in vitro using OCT ( $120 \mu \mathrm{m}$ for a $206-\mathrm{mmHg}$ pressure increase). In that study, the posterior cornea was visible only in two corneas, and no data were provided for lower pressures. We found an average decrease of $73.6 \mu \mathrm{m}$ for an increase of $40 \mathrm{~mm} \mathrm{Hg}$ in porcine corneas (average of 16 eyes). Cross- 
linking produced a reduction in the corneal thickness change by a factor of 2.28, consistent with an increase in corneal stiffness. The increase in the modulus of porcine corneal elasticity after CXL estimated from a simple model compares well with reports (a factor of $1.8 \mathrm{x}$ ) from corneal strip extensometry.[226] The absolute values obtained from inflation tests are lower than those obtained from corneal strip extensometry.

We measured changes in anterior and posterior corneal radius of curvature as a function of IOP variation (Figure 3.5 and 3.8). Changes in the posterior cornea tend to parallel the changes in the anterior cornea. We found highly significant differences between untreated and cross-linked corneas, consistent with an increase in corneal stiffness, particularly in the vertical direction. We found high meridional differences both on the effect of IOP on corneal curvature and on the effect of the cross-linking treatment. In untreated corneas, an increase of $40 \mathrm{mmHg}$ produced 8.26 times more increase in the vertical than in the horizontal anterior radius of curvature. The horizontal posterior radius was nearly not altered by IOP variations, while the vertical posterior radius changed by $500 \mu \mathrm{m}$ in the $0-40 \mathrm{mmHg}$ range. This result is in contrast to previous corneal strip extensometry experiments on specimens cut along the horizontal or vertical meridians of fresh porcine corneas, which showed the same stress-strain responses along the horizontal and vertical directions and only marginally stiffer (2-8\%) diagonally. [53] Microstructural studies of the fibrillar collagen structure in the porcine cornea show mainly a circumferential orientation, unlike other species (such as horse, cows or marmosets) that show dominantly a vertical orientation in the collagen fibrils or human where the stromal fibrils have a preferential orientation in the superior-inferior and temporal-nasal direction. [84, 131] The orientation-dependent corneal deformation found in our whole globe inflation experiments suggests that other factors beyond the orientation of collagen fibrils play a role in the anisotropy of the biomechanical response.

Cross-linking was much more effective in preventing an increase in 
Chapter 3. Biomechanical Changes after Collagen Cross-Linking from Porcine Eye Inflation Experiments

anterior corneal radius (both anterior and posterior, and immediately and 24-hour after) in the vertical than in the horizontal meridian - in fact we found some horizontal steepening with IOP in cross-linked corneas-. The inherent astigmatism of the porcine eye (on average the horizontal meridian was $2.19 \%$ flatter than the horizontal meridian) may play a role in the effective UV-irradiation treatment area (and therefore the relative exposure). However, differences of $710 \mu \mathrm{m}$ in the treatment area are not expected to result in these meridional differences in stiffness. Structural differences in the horizontal and vertical fibrils themselves, and a differential response to the formation of cross-linking bonds may be responsible for the observed meridional differences in the response to cross-linking.

\section{Implications of the results}

We have provided experimental data of the change in apex shift, corneal thickness, anterior and posterior corneal radius of curvature in aporcine eye inflation model, after CXL (and control eyes after epithelium removal and riboflavin instillation but not after UV-A irradiation). Although differences are expected on human corneas (particularly because of the relatively larger corneal cross-section treated) and in vivo, the complete experimental data set, along with estimations of the Young's modulus, are valuable input parameters in finite element models that will allow a better understanding and increased predictability of the cross-linking technique. To build a more sophisticated model with fewer limitations, we are planning further experiments with a high-resolution OCT and a larger lateral range that will allow viewing of limbal and scleral regions.

We have related some experimental findings on porcine corneas in vitro to clinical data found in patients. The use of a similar methodology on human corneal specimens, along with parameters obtained in vivo (particularly in eyes with corneal disorders susceptible to treatment) will provide further insight into the mechanism of cross-linking. Open questions such as the apparent anisotropy in the biomechanical response 
of the porcine intact cornea and in response to treatment, yet to be confirmed in humans and of particular interest in pathologic corneas, require combined studies of microstructural analysis of the collagen fibers and biomechanical approaches. 
Chapter 3. Biomechanical Changes after Collagen Cross-Linking from Porcine Eye Inflation Experiments 


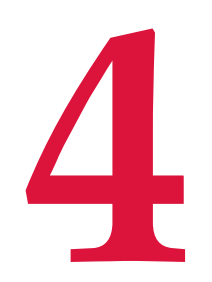

\section{Effect of hydration state and} storage media on corneal

\section{biomechanical response from in vitro inflation tests}

In the previous chapter we studied the difference between riboflavindextran and riboflavin-dextran + CXL treated corneas. Compared to virgin corneas in both conditions the corneal thickness is much lower due to the strong dehydration induced by dextran. This indicates that the biomechanical response measured might be different just because of the aplication of the photosensitizer solution. In this study we evaluate the 
Chapter 4. Effect of hydration state and storage media on corneal biomechanical response from in vitro inflation tests

effect of the corneal hydration state and different medical preservation solutions on their effect on the corneal biomechanical response. This is especially important in order to improve in vitro experiments on eyes obtained from an eye bank.

This chapter is based on the publication by S. Kling and S. Marcos, entitled "Effect of Hydration State and Storage Media on Corneal Biomechanical Response From in vitro Inflation Tests" in Journal of Refractive Surgery. This work was also presented in a oral contribution at the 2010 Euromech-518 Workshop "Biomechanics of the Eye" in London (UK).

The author of this thesis (i) did the sample preparation, (ii) performed the measurements, (iii) did the data analysis and (iv) prepared the manuscript in collaboration with S. Marcos. 


\section{Introduction}

Quantifying corneal biomechanical properties is important for the understanding of different corneal pathologies and treatments. Most experimental data of corneal biomechanics have been obtained through in vitro extensiometry measurements, where stress-strain functions are obtained from corneal strips[90, 226]. Alternatively, corneal inflation experiments in vitro[16, 56, 88] have allowed the analysis of the cornea in a condition closer to that of the living eye[151]. However, the experimental conditions (including time post-mortem, temperature and preservation media) affect the hydration state and microstructure of the cornea, and therefore likely play a role on the biomechanical properties estimates. $\mathrm{PH}$ and the osmotic tolerance of the corneal epithelium are considered to impact the corneal swelling susceptibility,[51] and swelling itself has been found to decrease the mechanical strength of the corneal tissue[2]. Also medical solutions, typically applied for preservation or treatments, are known to modify the corneal hydration state, both in vivo and in vitro (E. Spoerl, IOVS 1997, 38: ARVO Abstract 2339). This is in particular the case for Riboflavin-Dextran, used as photo-sensitizer in the UV collagen cross-linking procedure. UV-A collagen cross-linking is a technique aiming at increasing corneal rigidity, [195, 226] and is generally performed with a concentration of 0.125 Riboflavin in 20\% Dextran[194, 226] (although other Riboflavin concentrations[195] and hypotonic solutions have also been tested[79, 104]). In previous inflation experiments[109] we found a significant stiffening of the cornea, with the cross-linked corneas showing an increase by 1.58 in the estimated Young modulus. It is interesting to investigate to which extent the Riboflavin-Dextran solution alone induces a change in the biomechanical properties of the cornea, with respect to those of virgin eyes. The standard photosensitizer solution for cross-linking contains Dextran, a neutral polysaccharide, which is well-known for its dehydrating effect. Dextran is often used to dehydrate the corneal tissue after storage in eye-banks or to prevent 
Chapter 4. Effect of hydration state and storage media on corneal biomechanical response from in vitro inflation tests

swelling in in vitro experiments. Another medical solution is Optisol GS, one of the most widespread storage media for human corneas in eye banks for the preservation before transplantation. [233] Although the ultimate goal in many applications addressing corneal biomechanics is to obtain corneal biomechanical properties in vivo, in vitro measurements are an important step forward, particularly if they are tested in conditions close to those in vivo. With this study we investigate the differences in corneal deformation patterns upon changes in IOP across different hydration conditions of the corneal tissue. This will help us to identify the best preservation media or photo-sensitizer solution, as well as optimal baseline conditions for the investigation of the corneal tissue's biomechanical properties.

\section{Conditions and Protocols}

The experiments were performed using the whole eye glob holder (see chapter 2) and the automatized inflation system (see chapter sc:pressureSystem) in combination with the commercial Pentacam Scheimpflug imaging system 2.

30 fresh, enucleated porcine eyes (left and right eyes packed together) were obtained from a local slaughterhouse (prior to scalding) and used immediately, i.e. within 4 hours post-mortem.

Table 4.1: Summary of the different conditions studied.

\begin{tabular}{r|cc|cc|cc} 
& \multicolumn{2}{|c|}{$\begin{array}{c}\text { number } \\
\text { of eyes }\end{array}$} & \multicolumn{2}{c|}{$\begin{array}{c}\text { without } \\
\text { epithelium }\end{array}$} & \multicolumn{2}{c}{ application } \\
\hline time & Oh & $24 \mathrm{~h}$ & Oh & $24 \mathrm{~h}$ & Oh & $24 \mathrm{~h}$ \\
\hline virgin & 6 & 6 & no & yes & - & - \\
8\% Dextran & 6 & 6 & yes & yes & topical & topical \\
$20 \%$ Dextran & 6 & 6 & yes & yes & topical & topical \\
Riboflavin-Dextran & 6 & 6 & yes & yes & topical & topical \\
Optisol & - & 6 & - & yes & - & immersed
\end{tabular}

The eyes were separated equally into five groups and treated with 
different medical solutions (see Table 4.1 and chapter 2 for a more detailed description of the individual solutions).

NORMALIZED THICKNESS The corneal hydration state was estimated by the normalized thickness

$$
H=\frac{t h}{t h_{0}}
$$

where th is corneal thickness in treated eyes at different times and $t h_{0}$ is corneal thickness in virgin eyes at 0 hours. $\mathrm{H}=1$ refers to normally hydrated corneas.

Each enucleated porcine eye was imaged separately. Motion during the experiment was prevented by fixing the eyes in a customized eyeholder which could accommodate the individual differences in diameter across eyes, see chapter 2 . Within the holder, we oriented the eye similarly as it was naturally oriented in the pig, i.e. the longer side horizontally and the optical nerve head upwards, preserving the natural superior-inferior orientation. The experiments were performed with the porcine eye within a moist chamber, see chapter 2, to guarantee a constant humidity. To allow an undistorted Scheimpflug imaging a small window was cut in the box. Control measurements (moist chamber without eye) showed that relative humidity was kept constant at $48 \%$ $\left(23^{\circ} \mathrm{C}\right.$ room temperature). One entire inflation procedure took about 20 minutes. First, $15 \mathrm{mmHg}$ of IOP (assumed as the physiological IOP of pigs) were adjusted for reference. Then, IOP was increased at 5 $\mathrm{mmHg}$ steps up to $55 \mathrm{mmHg}$, and then decreased at 5-mmHg intervals. Each pressure step was held constant for 1 minute before a Scheimpflug image of the anterior segment of the eye was collected. This ensured that the cornea had adapted a constant shape before the measurement. Figure 2.9 summarizes the measurement set-up. Measurements were performed without pre-stressing $[20,86]$ to avoid the drastic changes in the mechanical relaxation behavior[27] (particularly the viscoelastic behavior) normally produced pre-conditioning[27] the tissue, and to allow evaluation of the corneal viscoelastic properties of the original 
Chapter 4. Effect of hydration state and storage media on corneal biomechanical response from in vitro inflation tests

Table 4.2: Corneal normalized thickness before measurements at $0 \mathrm{~h}$ and $24 \mathrm{~h}$.

\begin{tabular}{l|cc} 
& Oh & $24 \mathrm{~h}$ \\
\hline virgin & 1.00 & 1.17 \\
8\% Dextran & 0.54 & 0.74 \\
20\% Dextran & 0.60 & 0.75 \\
Riboflavin & 0.55 & 0.69 \\
Optisol & - & 1.05
\end{tabular}

first-stretch behavior in natural conditions. Also, the fact that in our inflation set-up the corneal tissue is loaded in the same direction as in vivo conditions and the globes are constantly exposed to the intraocular pressure makes it unnecessary to precondition the tissue, as it was never allowed to relax before the measurements, and to allow the fibers towards the load direction. [57, 208] These favorable conditions resulted in highly repeatable curvature and thickness deformation upon IOP variation, even without tissue pre-stressing.

\section{Results}

\section{Corneal Thickness}

The mean corneal thickness (measured over a central zone of $6 \mathrm{~mm}$ diameter) varied strongly across the different hydration conditions (Figure 4.1). The largest differences were observed between the dehydrated conditions (8\% and 20\% Dextran, Riboflavin-Dextran) and the other conditions (virgin, Optisol). The application of any of the Dextran solutions (30 minutes prior to measurements) produced a decrease to $56.5 \%$ (0h) and $72.7 \%(24 \mathrm{~h})$ in corneal thickness compared to the virgin cornea (see 4.2). The initial corneal thickness was found to be $510 \pm 24.4 \mu \mathrm{m}$ (0h) / $660 \pm 24.5 \mu m(24 \mathrm{~h})$ in Dextran treated eyes and $907 \pm 36.3 \mu \mathrm{m}$ (0h) / $1064 \pm 53.1 \mu \mathrm{m}(24 \mathrm{~h})$ in virgin eyes. The changes from 0 to 24 hours were very similar in all groups $\left(\Delta t h_{0 h-24 h}=-154 \pm 2.91 \mu \mathrm{m}\right)$, indi- 
cating that overnight swelling is independent of the prior state of corneal dehydration.

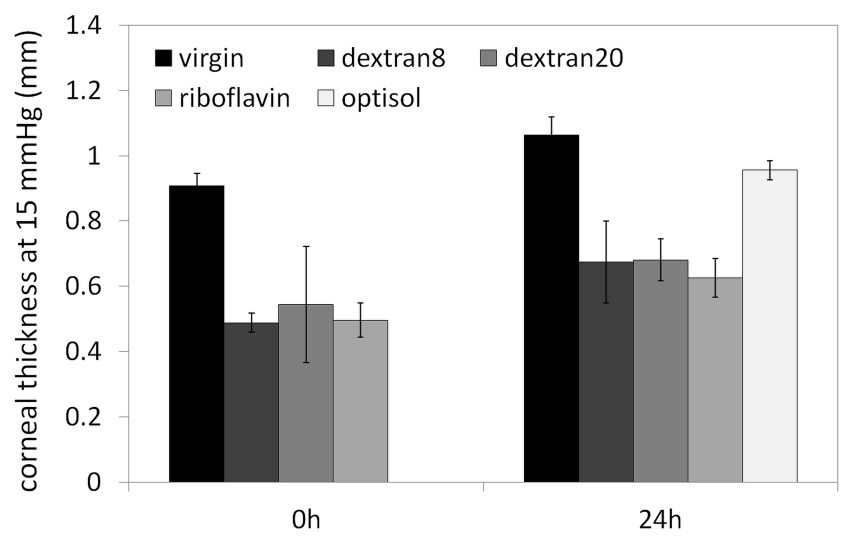

Figure 4.1: Initial corneal thickness before IOP variation for all tested conditions, at 0 hours and 24 hours.

Figure 4.2 shows the change in corneal thickness with IOP (with respect to the initial value at $15 \mathrm{mmHg}$ ) in all conditions (circles and triangles). An increase in IOP produced a reduction in corneal thickness in all groups, regardless the prior hydration state. The maximum mean thickness decrease in virgin corneas differed significantly from that found for $8 \%$ Dextran $(\mathrm{p}=0.04), 20 \%$ Dextran $(\mathrm{p}=0.001)$ and RiboflavinDextran $(\mathrm{p}=0.03)$ treated corneas, but not with respect to Optisol treated corneas $(\mathrm{p}=0.43)$. Measurements at $0 \mathrm{~h}$ and $24 \mathrm{~h}$ were statistically similar (CI: 95\%) for all conditions, except for Riboflavin-Dextran $(\mathrm{p}=0.02)$.

\section{Corneal Curvature}

Corneal curvature data were noisier than thickness data (mean $S D=$ $89.2 \mu \mathrm{m}$ versus $8.96 \mu \mathrm{m}$ ). 4.3 shows the changes in mean (anterior and posterior) corneal curvature as a function of IOP variation, averaged over $0 \mathrm{~h}$ and $24 \mathrm{~h}$ measurements. The variation of corneal curvature with IOP shows similar trends to those found for the thickness variations with 
Chapter 4. Effect of hydration state and storage media on corneal biomechanical response from in vitro inflation tests
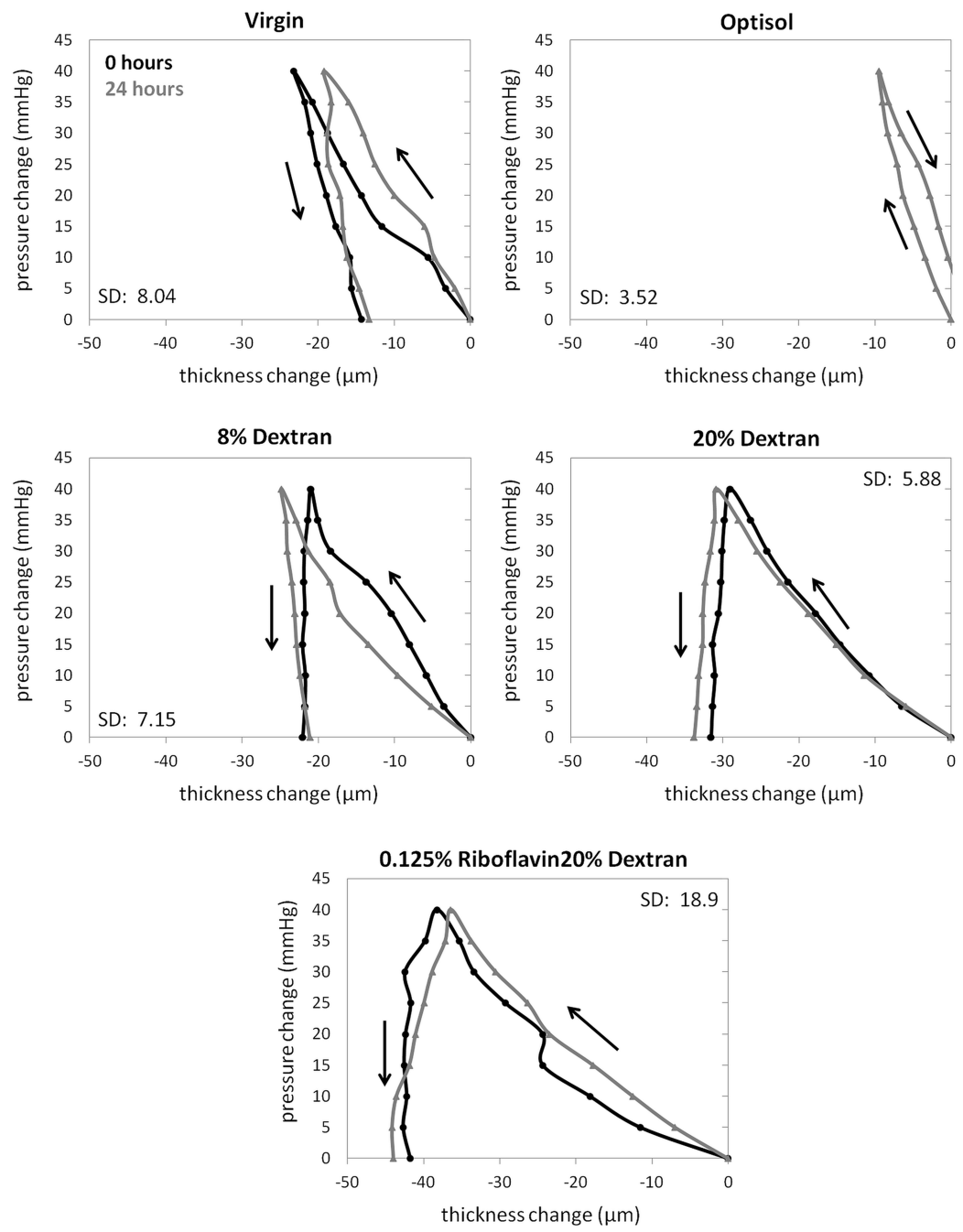

Figure 4.2: Corneal thickness response to IOP variation. Black represents 0 h data and grey $24 \mathrm{~h}$ data. IOP variation is respect to $15 \mathrm{mmHg}$ initial IOP. 
IOP: Virgin and Optisol corneas deformed less than corneas treated with Dextran, and showed less remaining deformation after IOP decreased back to physiological levels.

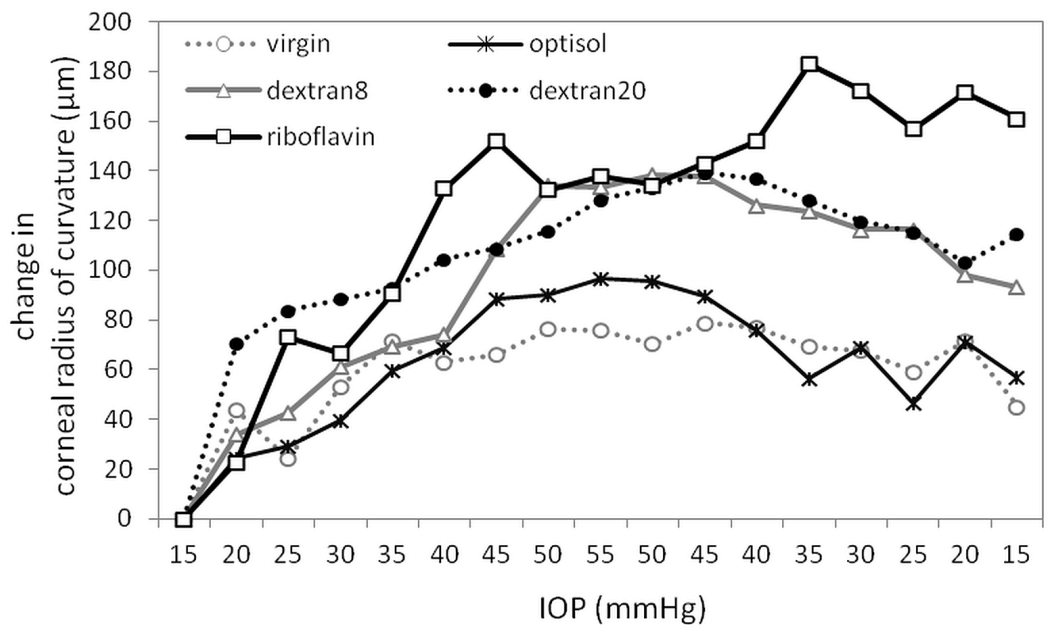

Figure 4.3: Changes in corneal radius of curvature as a function of IOP variation. Data are averaged of anterior and posterior changes, and $0 \mathrm{~h}$ and $24 \mathrm{~h}$ measurements.

\section{Corneal Hydration}

The corneal hydration states estimated for different treatments and times after enucleation are listed in Table 4.2. Corneas treated with Dextran showed a similar degree of dehydration, and virgin (and Optisol corneas) are about x 1.67 more hydrated. While hydration increased after 24 hours in all conditions (by x 1.3), the ratio of hydration between Virgin and Dextran-treated corneas remained constant.

\section{Epithelium and Corneal Transparency}

Virgin eyes could not be measured at 24 hours, as when IOP increased to $40 \mathrm{mmHg}$ the epithelium lost its transparency preventing the acquisition 
Chapter 4. Effect of hydration state and storage media on corneal biomechanical response from in vitro inflation tests

of Scheimpflug images of the posterior corneal surface. Also a custom spectral Optical Coherence Tomography system2 $[77,145]$ was tested and failed to obtain a feasible image. As soon as the epithelium had been removed, there was no further problem in obtaining high quality images from the anterior and posterior corneal surfaces. We also encountered epithelial transparency loss after storing eyes with epithelium for 24 hours in Optisol GS.

\section{Discussion}

We have investigated the biomechanical response of the cornea in a cycle of decreased and increased intraocular pressure in a whole eye inflation experiment, and found that different solutions typically used in storage or treatment of the cornea result in different corneal deformation patterns upon IOP variation. This suggests changes in the biomechanical properties of the cornea (elastic and viscoelastic properties) as a result of the modulation of corneal hydration produced by these solutions, and a potential impact of those solutions on the corneal micro-structure. The changes in corneal thickness as a function of IOP variation (Figure 4.2) reveal interesting differences across conditions. The slope of the curves in the IOP increasing range (from 15 to $55 \mathrm{mmHg}$ ) is related to the stiffness of the corneal tissue. Compared to the virgin cornea, Dextrantreated corneas showed a flatter slope and Optisol treated corneas a steeper slope. These results suggest that Dextran- treated corneas are less stiff than virgin or Optisol-treated corneas, although the initial corneal thickness is also likely to play a role in this variation. The most obvious differences across conditions occurred in the amount of hysteresis (related to the deformation remaining after the IOP variation cycle). A higher hysteresis was associated with a higher Dextran concentration, in comparison with virgin corneas. The presence of Riboflavin enhanced this effect further, while Optisol reduced it. The corneal deformation in the IOP decreasing range (from 55 to $15 \mathrm{mmHg}$ ) depends to a high 
degree on its viscoelastic properties (such as the relaxation time). A faster recovery should occur in virgin and Optisol-treated eyes than in Dextran-treated eyes. We have shown that the corneal hydration condition plays an important role in the biomechanical response of the cornea, as the different solutions affect corneal hydration differently. However, hydration alone is not the only cause for the observed differences in the biomechanical response of the cornea, as the measurements at 0 hour and 24 hours (which showed significant differences in the hydration state) only show slight differences in the deformation pattern with IOP. However, significant differences were observed across different treatment conditions. Although the water content of porcine corneas[81] is slightly lower compared to humans, the rate of dehydration produced by Dextran occurs similarly in human $[46,111]$ and porcine corneas. Interestingly, while the corneal swelling seems to occur similarly in all conditions after 24 hours (except to a less extent with Optisol), the response of corneal thickness upon varying IOP differed significantly across conditions. These results suggest that apart from the modulation of corneal hydration, the specific composition of the solutions has additional impact on the biomechanical properties of the cornea. According to Fratzl, the corneal tissue dries in two stages[69]. Initially, only the inter-fibril substance dehydrates; but then, after a certain level of dehydration is exceeded, the fibrils reduce in diameter. This model is consistent with our findings with Dextran. As dehydration of the extracellular matrix dominates overall corneal thickness, this probably happens up to a $510 \mu \mathrm{m}$ thickness (porcine corneas). This corneal thickness was consistent in corneas treated with Dextran at 0 hours, although it would be expected to be higher for higher Dextran concentrations. Beyond this first dehydration level, the level of subsequent fibril dehydration (following the model referred by Fratzl and Daxer [69] will be reached. Riboflavin is a smaller molecule (molar mass of $376.36 \mathrm{~g} / \mathrm{mol}$ ) than Dextran[95] (molecular weight from $10^{3}$ Daltons to $2 \cdot 10^{6}$ Daltons), and therefore may alter the structural properties of the collagen lamellae more easily. This could 
Chapter 4. Effect of hydration state and storage media on corneal biomechanical response from in vitro inflation tests

explain the different biomechanical behavior in comparison with the corneas treated with 20\% Dextran without Riboflavin. Also, Wollensak observed an increase in collagen fiber diameter after Riboflavin-Dextran UVA cross-linking [228] and a decrease in the corneal swelling,[222] although other studies on chemical collagen cross-linking (i.e. without Riboflavin as photosensitizer) reported reduced hydration of the collagen fibrils after cross-linking. $[8,134]$ We suggest that this increase in fibril diameter might come from the Riboflavin. An important implication of this study pertains to the design and evaluation of cross-linking treatments on corneal stiffening. Previous inflation experiments[109] showed a significant stiffening of the cornea, with the cross-linked corneas showing an increase by 1.58 in the estimated Young modulus. Similar to many other studies, the control condition involved de-epithelization of the cornea and Riboflavin-Dextran solution instillation. However, as the results from this study suggest an overall weakening of the corneal tissue after Riboflavin-Dextran instillation (flatter slope in 4.2), the CXL treatment in keratoconus eyes could be actually more effective when using another photosensitizer that does not soften the corneal tissue. The use of alternative photosensitizer solutions should be considered. Hypotonic solutions (e.g. $0.9 \%-\mathrm{NaCl}$ ) are already being studied in order to replace Dextran and to prevent the corneal dehydration and thinning during the treatment.[79] Our study suggests that not only Dextran, but also Riboflavin may be playing a role in the modulation of the biomechanical properties of the cornea. This study has important implications for the selection of the appropriate experimental conditions in experiments aiming at estimating biomechanical properties of the cornea. In many cases, particularly with eye-bank specimens, immediate access to tissue is not possible. We showed that none of the medical solutions containing Dextran, preserved its biomechanical response. This should be taken into account when performing biomechanical experiments on tissue that has been stored. The availability of experimental corneal deformation will allow to build more accurate analytical and numerical 
models, and increase the predictability of surgical results. In a first step, corneal deformation data can be used for inverse modeling to obtain the corresponding biomechanical parameters, for each condition. Corneal shape and thickness can serve as inputs for Finite Element Models. Both changes in corneal curvature and thickness showed similar same trends on the relative amounts of deformation and hysteresis. However, considering that the curvature deformation is more strongly influenced by the overall ocular geometry and by scleral properties, models can rely on corneal thickness changes as input data for future models, or more comprehensively, include experimental data on the scleral geometrical and mechanical properties to provide a complete model of eye inflation. 
Chapter 4. Effect of hydration state and storage media on corneal biomechanical response from in vitro inflation tests 


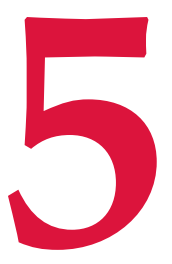

\section{Corneal biomechanical properties} from two-dimensional corneal flap extensiometry: application to UV-Riboflavin cross-linking.

In the previous chapter we demonstrated that hydration modifies the corneal biomechanical response. In this study we developed a new technique to measure the corneal stress-strain curve in thin two-dimensional flaps while controlling their hydration. This approach combines the advantages of previously used strip extensiometry and whole globe inflation technique, increases the resolution along the corneal thickness and allows 
Chapter 5. Corneal biomechanical properties from two-dimensional corneal flap extensiometry: application to UV-Riboflavin cross-linking.

controlling the hydration state.

This chapter is based on the publication by S. Kling et al, entitled "Corneal biomechanical properties from two-dimensional corneal ap extensiometry: application to UV-Riboflavin cross-linking" in Investigative Ophthalmology and Vision Science. Co-authors are H. Ginis and S. Marcos. This work was also presented in a poster contribution at the 2012 Annual Meeting of the Association for Research in Vision and Ophthalmology (ARVO) in Fort Lauderdale (Florida, USA).

The author of this thesis (i) implemented the experimental set-up in collaboration with H. Ginis, (ii) did the sample preparation, (iii) performed the measurements, (iv) did the data analysis and (v) prepared the manuscript in collaboration with S. Marcos. 


\section{Introduction}

Knowledge of corneal biomechanical properties is critical for modeling the biomechanical response of corneal tissue in disease (i.e. keratoconus, a progressive corneal disease that debilitates corneal tissue) and treatment (i.e. intrastromal ring segments, corneal cross-linking or incisional surgery) in order to increase the predictability of surgical outcomes. Various methods have been used in the past to estimate the modulus of elasticity (Young modulus) of the cornea. The most widespread method is strip extensiometry[56, 58, 224, 226] followed by corneal button inflation[16, 112] and whole-globe inflation.[56, 88, 109, 123] In these techniques a load is applied (typically along one axis - in strip extensiometry - or radially by increasing intraocular pressure in inflation techniques). The strain is then measured from the lateral elongation, axial apex displacement,[56] shift of mercury droplets attached on the corneal surface,[16] or from changes in the corneal radius of curvature $[109,158]$ upon the application of stress. New techniques for the estimation of corneal biomechanical properties from direct measurements of corneal deformation upon air puff applanation have also been recently presented.[45, 82] In all in vitro corneal biomechanical measurements corneal hydration plays a role, which is known to affect the tissue's biomechanical response.[87] Also, different corneal treatments effect corneal hydration and biomechanical properties differently (Kling S., IOVS, 2010, Corneal Biomechanical Response to Intraocular Pressure Changes From Scheimpflug and Anterior Segment OCT, ARVO E-Abstract, 4628/D750). UV riboflavin cross-linking (CXL) is an emergent technique for treatment of keratoconus, which aims at stiffening the corneal tissue. The increase in corneal rigidity gained with this treatment is believed to result from the reaction of the photosensitizer (riboflavin) with UV light, which creates radicals that induce additional cross-links between collagen fibrils, probably interhelically, intrahelically and intermicrofibrillary.[1, 228] Strip extensiometry stress-strain experi- 
Chapter 5. Corneal biomechanical properties from two-dimensional corneal flap extensiometry: application to UV-Riboflavin cross-linking.

ments showed an increase in corneal rigidity at several months after CXL treatment in human[224], porcine[89] and rabbit[183] models. Wholeglobe inflation experiments also showed an increase in corneal rigidity in eyes in vitro immediately after CXL.[109] The biomechanical response estimated from some of the previous methods may be affected by corneal shape (geometry), thickness, hydration state (in vitro) and intraocular pressure (in vivo). In this study we developed a new two-dimensional stress strain system that allows maintaining the original stress distribution along the corneal flap, while guaranteeing that corneal hydration and thickness are equal for all samples. This allows an accurate comparison between individual flaps of a certain layer, as well as a precise analysis of the treatment effects on a few corneal layers. To prove its application we evaluated the change in corneal rigidity following CXL treatment.

\section{Protocols}

The 2D-flap holder (see chapter 2) was used in combination with the commercial Tracey ray tracing aberrometer (see chapter 2). The experimental data were analyzed using the analytic procedure described in chapter 2.

In-vitro experiments were performed in order to measure the elasticity of corneal flaps and to investigate the effect of changes in rigidity due to CXL. Measurements were performed on fresh enucleated porcine eyes (before and immediately after CXL performed in vitro), as well as in New Zealand rabbit eyes ( 1 month after CXL performed in vivo). Measurements in porcine eyes allowed establishing the technique, while measurements in rabbit eyes allowed closer conditions to the clinical procedure. Porcine eyes were obtained from a local slaughterhouse and used within 24 hours. Rabbits were obtained from a certified farm at the age of 3 months (approx. $2 \mathrm{~kg}$ weight). After performing CXL treatment in the left eye, the 106 rabbits were housed in animal facilities where they were cared. A total of 13 porcine eyes and 4 rabbit 
eyes were tested. The protocols adhered to the ARVO guidelines for animal research and had been approved by the Institutional Review Board. In porcine eyes in vitro, first the epithelium was removed with a hockey epithelium removal knife and 20\% Dextran (Sigma-Aldrich D8821) solution was applied for 40 minutes. Then the intraocular pressure was adjusted to physiologic value $(15 \mathrm{mmHg}$ ). A flap was cut with a mechanical Carriazo-Pendular microkeratom (see chapter 2) and mounted in the custom holder (see chapter 2). Ultrasonic pachymetry was performed in the intact eye before cutting the flap, and then, the corneal bed was measured again in order to estimate the thickness of the removed flap. Measurements were conducted in the untreated flap (see below) and then CXL was applied on the corneal flap in-situ. Ultraviolet light $\left(370 \mathrm{~nm}, 3 \mathrm{~W} / \mathrm{cm}^{2}\right.$ ) was applied using homogenous circular illumination (entire cornea) during 30 minutes. For control purposes, measurements were also obtained on non UV- irradiated flaps, 30 minutes after the initial measurements. Rabbits were anesthetized using $1 \mathrm{ml}$ Ketamine hydrochloride 10\% $+2 \mathrm{ml}$ Xylazine 2\%. CXL was performed following standard clinical conditions (first 30 minutes riboflavin instillation, followed by 30 minutes $367 \mathrm{~nm}$ UV-light exposure, while continuing riboflavin instillation every 3 minutes). Left eyes were treated, and the right eyes were left untreated for control. Rabbits were euthanized one month after treatment. Corneal flaps were created and excised immediately after animal sacrifice, mounted in the flap holder and further handled as the in-vitro flaps. Rabbit eyes were examined with a confocal light microscope (HRT, Heidelberg Engineering, Heidelberg) at different times: before treatment, immediately after CXL, one day after CXL and one month after CXL. Flaps were treated with CXL in-situ and in-vivo. An analytical model was applied to estimate tissue elasticity in non-treated corneas and after CXL. 
Chapter 5. Corneal biomechanical properties from two-dimensional corneal flap extensiometry: application to UV-Riboflavin cross-linking.

\section{Stress-strain cycles}

Corneal flaps were mounted in a chamber (for a more detailed description see chapter 2) connected to a pressure system that applied the force to stretch flaps of porcine and rabbit corneas. Measurements were conducted for a series of pressures, for each flap and condition. Initially, the pressure in chamber 1 was set equal to pressure in chamber 2 (1013 $\mathrm{hPa}$ ). Then one preconditioning cycle was performed up to the pressure of $35 \mathrm{mmHg}$. Pressure was increased up to $30 \mathrm{mmHg}$, and then decreased, in about $5 \mathrm{mmHg}$ steps. After that measurements were performed for two inflation deflation cycles. The geometry of the flap was assessed using a commercial ray tracer (2) for each pressure. Flaps were mounted in the holder as shown in Figure 2.6, and the system used in place of the eye for aberrometry measurements. Changes in the low order aberrations are related to changes in the curvature and astigmatism of the flap surface (see 2). The measurement wavelength was $670 \mu \mathrm{m}$. Zernike coefficients (up to the 7th order) were obtained for a pupil diameter of $2.5 \mathrm{~mm}$, and the low order terms (defocus and astigmatism) were used for analysis. Ray tracing measurements were obtained during two inflation cycles for each flap and condition, which in total took about 20 minutes. Thereby measurements were performed in approximately $5 \mathrm{mmHg}$ pressure steps.

\section{Data Analysis}

The flap deformation was monitored with a ray-tracing aberrometer (see chapter 2). The amount of defocus in the artificial eye is related to the stretching of the corneal flap. The higher the pressure in Chamber 1 , the more curved the flap and the higher the change of the system's refractive power. Due to small deformation produced by the applied pressures, it can be assumed that the flap is deformed spherically. This approximation is further justified as we selected a central portion of the flap for measurement and analysis. Zernike coefficients $Z_{2}^{0}$ (defocus term) and $Z_{2}^{2} / Z_{2}^{-2}$ (astigmatism at $45^{\circ} / 90^{\circ}$ terms) were analyzed. The defocus 
term was used to calculate the surface area of the flap as a function of pressure (see chapter 2).

\section{Results}

\section{Flap Pachymetric and Microscopic Observations}

Corneal flap thickness of porcine and rabbit corneas ranged between 80 and $120 \mu \mathrm{m}$. After manual excision of the hinge, a 6-mm circular portion of the flap was mounted and measured. In compliance with other studies on confocal microscopy[78] we observed highly reflective structures in the rabbit mid stroma one month after CXL (see Figure 5.1). Interestingly, these structures could not be observed immediately after, or one day after CXL.

\section{Defocus Aberration}

Figure 5.2 shows the change in the defocus term from the Zernike polynomial expansion as a function of pressure in Chamber 1 from untreated and treated corneas in porcine (Figure 5.2A) and rabbit (Figure 5.2B). Black symbols represent the control measurement, while white symbos stand for the cross-linked condition. Data are the average across 13 porcine corneas, pre and post-CXL, and across 8 rabbit corneas, with (right) and without CXL (right) (Figure 5.2B).

Defocus increased linearly with increasing pressure in all conditions. Regression lines to control (bold) and CXL (thin) data show a positive slope. The lower the slope, the smaller the deformation and the stiffer the corneal flap. In porcine corneas (Figure 5.2A), the variation of the defocus with pressure in non-treated flaps was not statistically significantly different to CXL flaps $(\mathrm{p}=0.76)$. In rabbit corneas (Figure 5.2B) one month after CXL, the slope of the defocus term variation was significantly steeper in flaps from non-treated corneas than in flaps from cross-linked corneas $(\mathrm{p}=0.026)$, consistently with an increase in corneal 
Chapter 5. Corneal biomechanical properties from two-dimensional corneal flap extensiometry: application to UV-Riboflavin cross-linking.
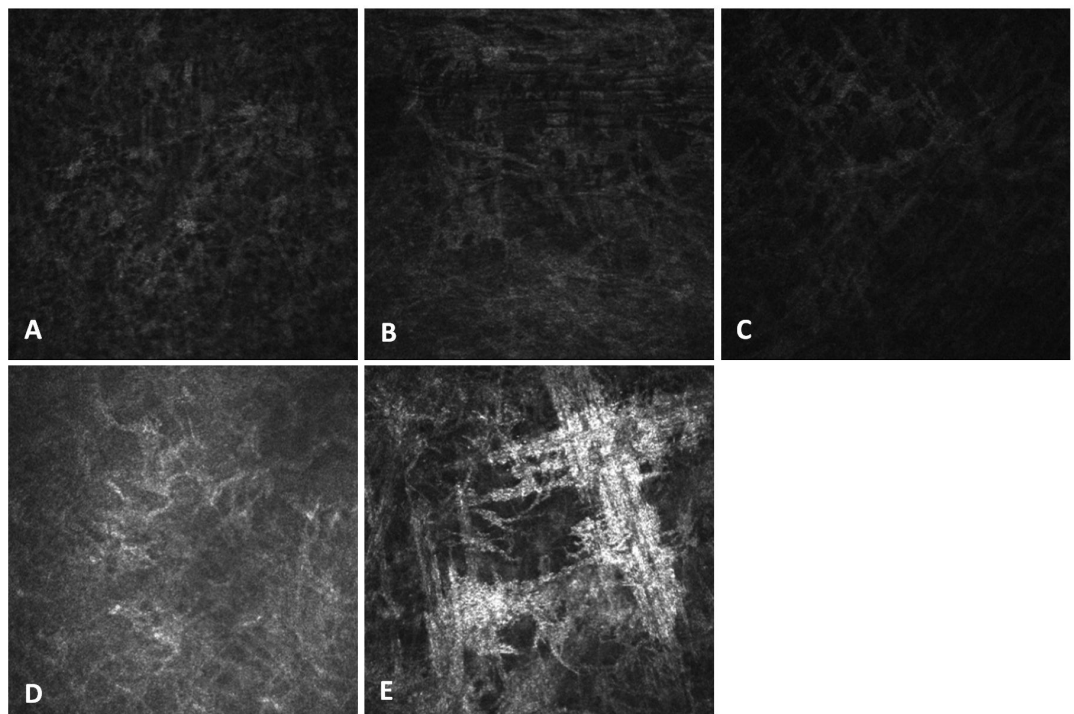

Figure 5.1: Confocal microscopy images comparing the mid stroma (about $130 \mu \mathrm{m}$ depth) in three conditions (rabbits): (A) virgin cornea, $(B)$ riboflavin instillation, $(C)$ immediately post cxl, (D) one day post cxl, (E) one month post cxl. 
rigidity after CXL. Variability across samples was $0.22 \mathrm{D}$ (non-treated)

- pre 0 post

A Pigs: immediately after in-vitro CXL

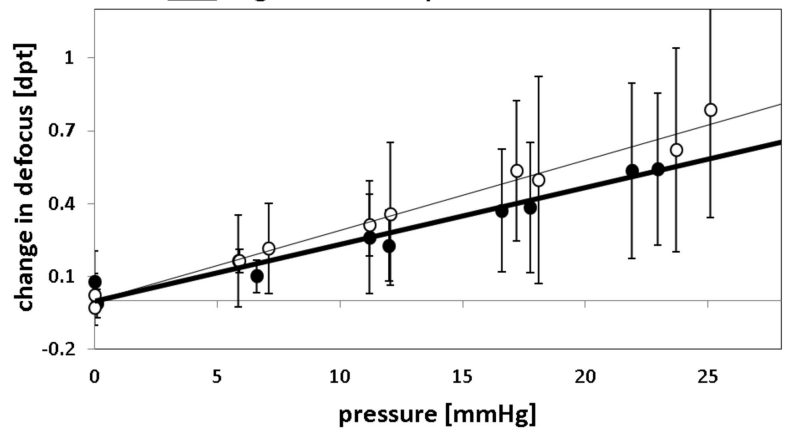

B Rabbits: 1-month after in-vivo CXL

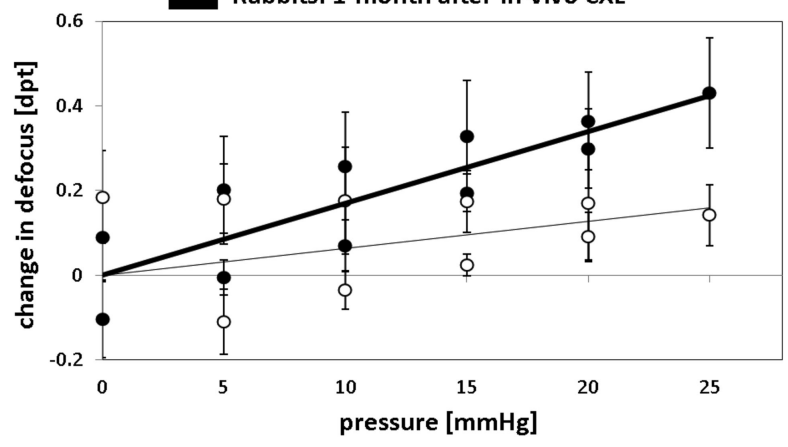

Figure 5.2: Changes in defocus as a function of pressure on the flap in pig eyes (A) and rabbit eyes (B) pre and post-CXL (in vitro CXL in pigs and in vivo CXL in rabbits).

and 0.26 D (CXL) in the in-situ condition, and 0.10 D (non-treated) and 0.08D (CXL) in the in-vivo condition. Porcine corneas showed a slightly flatter slope before treatment than immediately after CXL (23.4 versus $28.8 \mathrm{mD} / \mathrm{mmHg}$ ). However, the difference was not statistically significant. In rabbit eyes the slope in the non-treated eye $(17.0 \mathrm{mD} / \mathrm{mmHg})$ was significantly steeper $(\mathrm{p}=0.105)$ than one month after CXL (6.36 $\mathrm{mD} / \mathrm{mmHg}$ ). In certain conditions the flap geometry variation with pres- 
Chapter 5. Corneal biomechanical properties from two-dimensional corneal flap extensiometry: application to UV-Riboflavin cross-linking.

sured differed between the increased or decreased pressure. In porcine corneas neither the non-CXL nor the CXL flap showed this effect (control: $\mathrm{p}=0.338$ / CXL: $\mathrm{p}=0.509$ ). However in rabbit corneas, there was a significant shift in defocus (control: $p=0.032$ / CXL: $p=0.007$ ) after the increased/decreased pressure cycle: $0.19 \mathrm{D}$ in control flaps and $0.39 \mathrm{D}$ in CXL flaps (at $0 \mathrm{mmHg}$ pressure).

\section{Asitgamtic Aberration}

Mean astigmatism was modest, $0.55 \mathrm{D}$ (pre) and $0.47 \mathrm{D}$ (post) in porcine eyes and 0.74 D (non-treated) and 0.34 D (cxl) in rabbit flaps, and did not change significantly with pressure variation. In rabbit flaps a small decrease was observed after CXL.

\section{Young's Modulus}

\section{-, • pre $\quad---, 0$ post}

A Pigs: immediately after in-vitro CXL

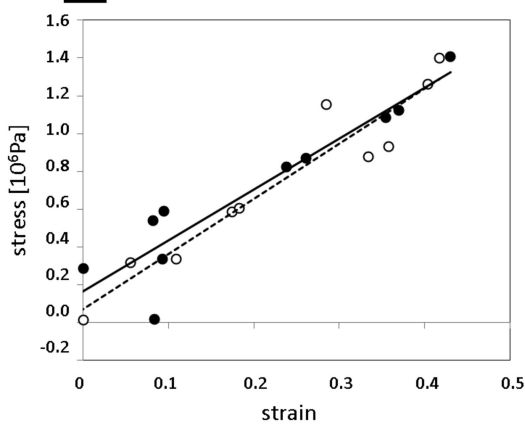

B Rabbits: 1-month after in-vivio CXL

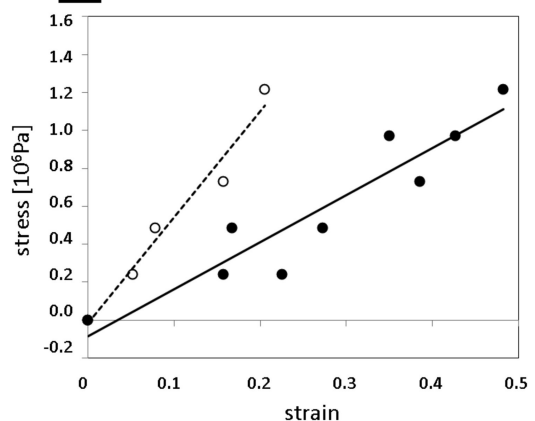

Figure 5.3: Stress-strain diagrams in pig eyes (A) and rabbit eyes (B), pre and post-CXL (in vitro CXL in pigs and in vivo CXL in rabbits). The Young modulus was estimated as the slope of the linear regressions.

Young's moduli were calculated for the different conditions. The average Young modulus in porcine flaps was $2.29 \pm 1.63 \mathrm{MPa}$, with no 
significant differences between pre and post in-situ CXL. The average Young's modulus of rabbit flaps was $1.98 \pm 0.40 \mathrm{MPa}$ and increased significantly $(\mathrm{p}=0.003)$ one month after in-vivo CXL $(4.83 \pm 1.32 \mathrm{MPa})$. Figure5.3 shows the stress-strain diagram, from which the slopes were used to calculate Young's modulus.

\section{Discussion}

We report a new 2D-flap extensiometry technique, which allows measuring corneal elasticity parameters, minimizing the effects associated with corneal hydration, among others. This method has proved sensitive to detect differences across conditions, and could be used to investigate corneal biomechanical properties at different corneal depths. While in the current study we used a mechanical microkeratome (which limited the thickness accuracy to which the flaps could be cut), the use of femtosecond laser would allow excising flaps of more resolved thickness and at different depth positions. The method could therefore be used to assess biomechanical properties of different corneal layers (i.e. anterior and posterior stroma). This information is valuable in spatially resolved finite element modeling analysis of the corneal biomechanical response. We evaluated the potential of the technique on corneal flaps from a porcine model, and investigated the biomechanical changes in the cornea 1-month after CXL in rabbit eyes. The estimated values of Young's moduli (porcine flap: 2.29 $\mathrm{MPa}$, rabbit flap: $1.98 \mathrm{MPa}$ ) fall within the ranges reported in literature, although these vary more than two orders of magnitude depending of the study.[16, 82, 88, 89, 107, 112, 113, 215, 224, 226] As a reference, in-vitro strip extensiometry experiments estimated a Young's modulus of 11.1 MPa for rabbit corneas and of 1.5 MPa for porcine corneas. [224, 226] An in-vitro button inflation study reported a Young's modulus of 2.87 - 19.5 MPa for the human cornea,[88] and a recent study using in-vitro whole globe inflation in porcine eyes reported a Young's modulus of 1.11 MPa (Kling S, et al. IOVS 2010;51:ARVO E-Abstract 
Chapter 5. Corneal biomechanical properties from two-dimensional corneal flap extensiometry: application to UV-Riboflavin cross-linking.

4628). Ultrasound measurements on in-vitro human corneas provided a value of 5.3MPa,[183] and an in-vivo approach based on the deformation with applanation tonometry $29 \mathrm{MPa}$ in humans. [165] The large differences likely arise from the different working principles of the techniques, and the hydration condition in which corneal tissue is measured. The proposed 2D-flap extensiometry technique reduces the variability by controlling the flap thickness and avoiding a major role of hydration. The technique therefore combines several advantages from previous methods. First, it guarantees a very similar distribution of the acting force (pressure) to the in-vivo condition, similar to button or whole-globe inflation. Second, the rigid circular fixation of the flap allows an accurate analysis of the corneal expansion, similarly to strip-extensiometry, but preserving a more realistic geometry and the actual orientation of the collagen fibers. The increase in corneal stiffness found after CXL in rabbits (x 2.43) is consistent with the literature. Previous studies reported an increase of corneal stiffness by a factor of $1.58-1.8$ (in pigs), by 4.5 (in humans) and by 1.6 (in rabbits).[109, 224, 226] A study of the long-term effects of CXL in rabbits (measured before and immediately after, 3 and 8 months after CXL) showed a high stability in the post-CXL corneal stiffness (a constant pre/post Young modulus ratio over time, by 1.6).1 While our method confirms the increase in corneal rigidity in rabbit corneas after 1 month post-CXL, we did not find a significant increase in porcine corneas immediately after CXL. This differs from previous studies on rabbit and porcine corneas, using strip extensiometry[226] and whole eye inflation.[109] In our study corneas were treated as a thin flap in situ, in contrast to all previous studies (and to the experiments in rabbit corneas in the current study) where the treatment was done on the entire corneal thickness, following standard clinical procedures. The in situ treatment in the first experiment was specifically chosen to allow a direct comparison with the biomechanical response of the pre-operative flap. The drastic corneal thinning (associated to the dehydration produced by Riboflavin),[108, 109] also observed during the CXL treatment,[114] 
would have produced a higher indetermination in the flap thickness cut. However, differences in the treatment protocol with respect to the clinical standard may have resulted in a less effective treatment. On the other hand, different results in flap extensiometry between in-vitro and in-vivo CXL could indicate a direction selective formation of cross-links: We can speculate that CXL may increase the number of bonds between different layers to a larger extent than the number of bonds within a single layer. In the current flap experiment mainly cross-links within a few layers carry the pressure load and flap thickness is not affected by hydration, suggesting that CXL might increase stiffness mainly in the transversal stretching direction. Additionally, other processes at the tissue level (e.g. wound healing) could contribute to the increase in corneal rigidity after CXL, as structural changes (highly reflective structures, Figure5.1) were observed to appear simultaneously with an increase in corneal rigidity. In a previous study in porcine eyes in vitro [109] we showed stronger stiffening effects of CXL in the horizontal than in the vertical direction. In the current study a slight reduction of corneal astigmatism with CXL was observed in rabbits, and has also been reported clinically in patients. [165] As a side effect, we observed that porcine corneal flaps did not show differences in the variation of the geometry with increased/decreased pressure, whereas in rabbit flaps a hysteresis was apparent. The current study on cornel flaps suggests that the spatially resolved analysis (in this case in thin corneal layers) of the processes occurring in CXL may give insights into the understanding of its mechanisms. The use of new techniques (such as second harmonic microscopy[23]) that allow visualization of the tissue, particularly collagen, at its structural level, in combination with the presented 2D-flap approach to exclude the effect of hydration may lead to interesting advances in the future. 
Chapter 5. Corneal biomechanical properties from two-dimensional corneal flap extensiometry: application to UV-Riboflavin cross-linking. 


\section{6}

\section{Brillouin microscopy of collagen}

\section{cross-linking: non-contact depth-dependent analysis of corneal elastic modulus}

The measurement of the corneal biomechanical properties in vitro are important, however it is of great clinical interest to determine the corneal rigidity in vivo. In this study we apply a non-contact Brillouin microscopy technique and demonstrate its sensitivity to distinguish corneas treated with different cross-linking protocols by their elasticity map.

This chapter is based on the publication by G. Scarcelli, S. Kling et 
Chapter 6. Brillouin microscopy of collagen cross-linking: non-contact depth-dependent analysis of corneal elastic modulus

al, entitled "Brillouin microscopy of collagen cross-linking: non-contact depth-dependent analysis of corneal elastic modulus" in Investigative Ophthalmology and Vision Science. Co-authors are E. Quijano, R. Pinedo, S. Marcos and S.H. Yun.

The author of this thesis (i) did the sample preparation, (ii) performed the measurements in collaboration with G. Scarcelli and (iii) revised the manuscript. 


\section{Introduction}

The decrease of corneal mechanical stability plays a critical role in the onset and progression of keratoconus and post-LASIK ectasia[49]. Corneal collagen cross-linking (CXL) is a promising treatment that aims at stopping the progression of ectasia by increasing corneal stiffness[221]. CXL induces the formation of covalent bonds between collagen fibers in the corneal stroma by photoactivation of a photosensitizer such as riboflavin. The increased number of the crosslinks increases the elastic modulus of the corneal tissue. CXL has been approved in Europe and is under clinical trials in the United States. The majority of CXL procedures follow the original "Dresden" protocol described by Wollensak et al.[226]. Over the past decade, studies following the long-term clinical outcome of the treatment have shown that the CXL procedure effectively stops progression of ectasia in the majority of patients[26, 165, 220]. Recently, however, a great deal of interest has been placed towards devising new CXL protocols to minimize the damage to keratocytes and to reduce the recovery time post intervention[80, 120, 224]. In this respect, a central role is played by the epithelium: because the epithelium represents a major barrier for the diffusion of photosensitizers into the stroma, the standard Dresden protocol involves the removal of the epithelium which results in delayed recovery and increased risks of infections; novel CXL procedures try to solve this issue by chemically loosening epithelial junctions [224, 232], or by custom epithelial debridement[120]. Despite the advance in CXL, it has been difficult to measure, monitor, and optimize the defining feature that drives the clinical outcome of the different CXL protocols, i.e. corneal mechanical stiffening, due to the lack of non-invasive mechanical characterization tools able to assess the performance of different protocols in vivo. Mechanical measurements are traditionally macroscopic and destructive [120]. Recently, a widespread effort to achieve a non-invasive test of corneal mechanical properties has been put forward. An ocular response analyzer measures corneal 
Chapter 6. Brillouin microscopy of collagen cross-linking: non-contact depth-dependent analysis of corneal elastic modulus

hysteresis[125]. Corneal hysteresis has been shown to correlate with CXL and advanced keratoconus[185, 199], but its clinical usefulness remains questionable[66, 67]. Anterior-segment imaging combined with an air puff has allowed dynamic measurements of corneal deformation, showing different deformation parameters in untreated corneas and crosslinked corneas[45]. This technique allows for in vivo measurements, but it remains challenging to determine the corneal biomechanical properties from the deformation images due to the contributions of the corneal geometry and intraocular pressure among other factors[45]. Other techniques based on ultrasound [85, 215] are under development[68, $76,206]$. Currently no clinical device is capable of directly measuring the mechanical properties, such as the elastic modulus, of corneal tissue. Recently, the feasibility of Brillouin microscopy for mapping corneal modulus in three dimensions with a high spatial resolution has been demonstrated $[177,180]$. This optical technique is noninvasive and does not involve structural or mechanical deformation of the cornea. In the study described here, Brillouin microscopy is applied to investigate the biomechanical properties of the cornea after various riboflavin-mediated CXL procedures. This study evaluates the differential effect of light dose, photosensitizer soaking, hydration and epithelial debridement on the mechanical outcome of CXL protocols. A Corneal Stiffening Index (CSI) is introduced as a quantitative metric to compare the mechanical outcome of a CXL protocol with that of the standard Dresden protocol. The results provide novel insights on the mechanical effects of CXL procedures and demonstrate Brillouin microscopy as a valuable tool for assessing CXL mechanical performance.

\section{Protocol}

The Brillouin microscopy set up described in chapter 2 was used to measure the corneal elasticity profile for different treatment protocols of cross-linking (epithelium-off, see chapter 2 versus epithelium-on, see 
chapter 2) and for different illumination and presoaking times.

Porcine eyes were obtained 2-4 hours post mortem and kept on ice during transport and storage until the starting of the experiments. The whole eye was placed in a chamber holder and gently flattened over a plastic dish to facilitate optical illumination/detection. Flattening the cornea did not alter its mechanical properties, as confirmed by control experiments of unflattened eyes resting suspended in a bath of saline solution or mineral oil. All reported experiments were conducted within 2 hours from tissue arrival. Brillouin measurements were obtained immediately following treatment, and typically took 20 minutes. Storage time (from tissue arrival until treatment) and measurement time (which could cause some evaporation) were found to have a negligible effect on corneal thickness. Also, differences in Brillouin modulus under similar treatment conditions, but slightly different times post-mortem (within the same session) were found to be negligible. On the other hand, soakingtimes and the solution used in the photosensitizer produced significant changes in thickness and Brillouin modulus.

To single out this factor, we performed sham control experiments where the entire eye followed a similar soaking protocol, but only half of the eye was exposed to UV light. Measurements were performed for both, the epi-off protocol (30-min soaking with Riboflavin/Dextran solution) and the epi-on protocol (30-min soaking with Riboflavin/PBS-BAC-NaCl solution). In these sets of eyes ( $\mathrm{N}=2$ for each condition), after soaking, one-half of the eye was blocked and the other half irradiated with UV light for 30 minutes. These controls guarantee identical timing, protocols and solution-dependent hydration states for a given condition, with the UV-irradiation being the only distinctive parameter.

A custom-made chamber held the corneas gently flattened onto a plastic dish in front of the Brillouin system so that the laser light accessed the sample in an inverted configuration through the flattened surface. 
Chapter 6. Brillouin microscopy of collagen cross-linking: non-contact depth-dependent analysis of corneal elastic modulus

\section{Rheometry}

For shear rheometry, a standard stress-controlled rheometer was used (AR-G2, TA Instruments) with 8-mm-diameter parallel-plates. At about 100 £gm pre-compression, first a strain sweep $(0.01 \%$ to $0.5 \%)$ was performed to determine the region of linear elastic regime; then, frequency sweeps from 0.1 to $10 \mathrm{~Hz}$ with $0.1 \%$ strain amplitude at $23^{\circ} \mathrm{C}$ were carried out. The data reported here refer to the shear modulus measured at $0.2 \mathrm{~Hz}$.

\section{Results}

\section{In situ mechanical characterization of corneas after stan- dard CXL}

Figure 6.1 shows the measurement results by Brillouin microscopy for the standard CXL procedure. Porcine corneas crosslinked following epithelial debridement, $30 \mathrm{~min}$ pre-soak and using $30 \mathrm{~min}$ of UV light exposure are compared to the untreated controls. Figure $6.1 \mathrm{a}-\mathrm{b}$ show representative crosssectional images ( $\mathrm{x}-\mathrm{z}$ plane) of the central portions of the cornea. The color-coding of Brillouin image reveals the depth-dependent variation of Brillouin shift (and elastic modulus) and the remarkable stiffening induced by cross-linking. For better visualization of the depth-dependence of corneal elasticity, the depth-profiles of Brillouin shift were computed from the cross-sectional images by averaging over the transverse axis. Figure $6.1 \mathrm{c}$ shows the representative depth profiles for both treated and untreated corneas. For each sample group, the depth profiles were highly consistent from sample to sample. The profiles clearly show the distinctly different modulus values between crosslinked and un-treated corneas. The depth-averaged modulus in each of the anterior, mid and posterior regions was calculated. A total of 6 crosslinked corneas and 3 untreated controls were used for this analysis. The increase in the mean modulus is remarkable in the anterior third of the cornea (unpaired t-test, 

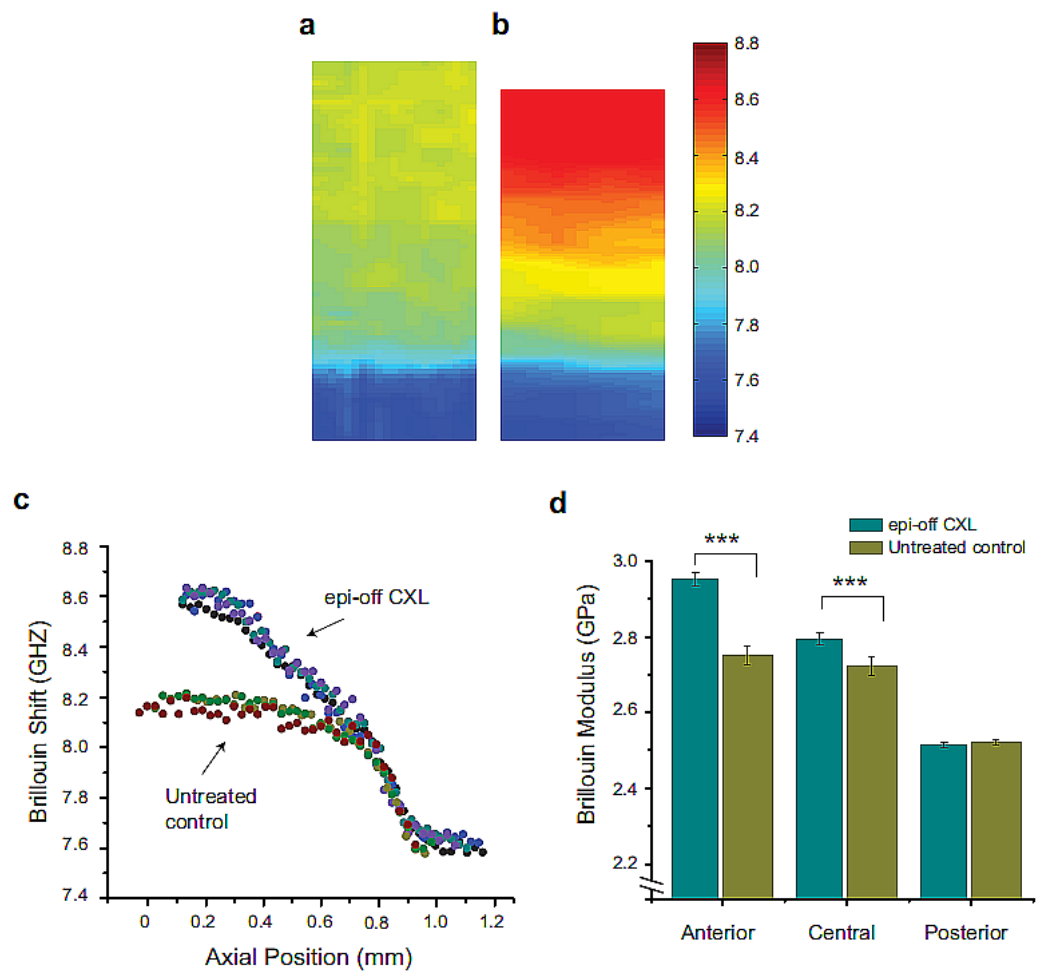

Figure 6.1: Brillouin mechanical characterization of standard epi-off CXL procedure. (a) A representative cross-sectional Brillouin image of normal porcine cornea. (b) A Brillouin image of the cornea after the standard CXL. The horizontal and vertical span is $0.05 \mathrm{~mm}(x)$ by $0.8 \mathrm{~mm}(z)$ in a and $b$. (c) Brillouin depth profiles of both crosslinked and untreated corneas. (d) Mean Brillouin modulus of the anterior, mid-, and posterior regions for the crosslinked $(N=6)$ vs. untreated corneas $(N=3) .{ }^{* * *} P<0.005$. 
Chapter 6. Brillouin microscopy of collagen cross-linking: non-contact depth-dependent analysis of corneal elastic modulus

$p<0.005)$, and it is less substantial but still highly statistically significant in the mid-stromal region (unpaired t-test, $p<0.005$ ). No changes were detected in the posterior stromal region. These features are consistent with previous literature and CXL modeling[113, 182].

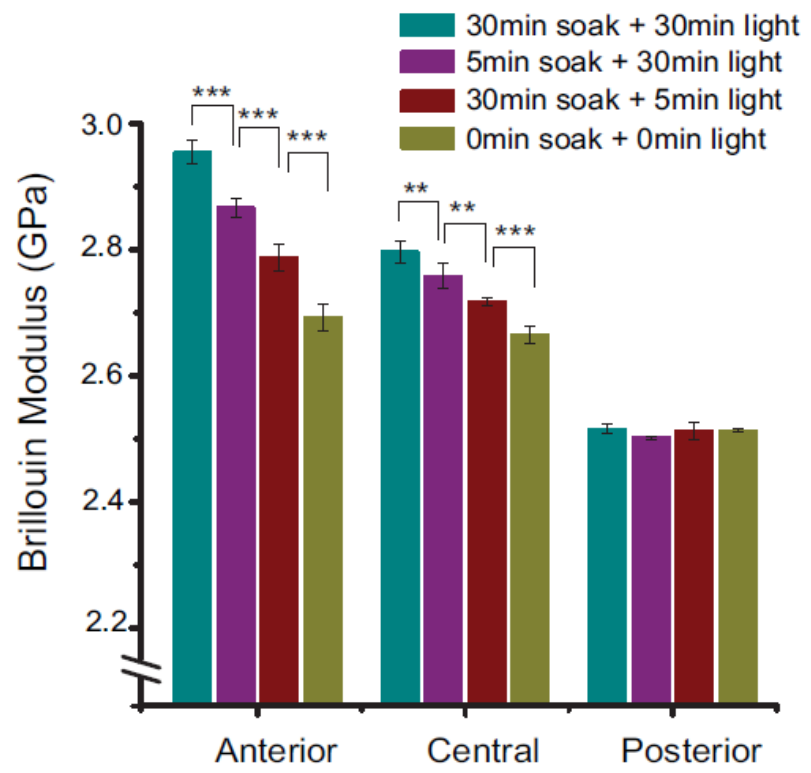

Figure 6.2: The effects of the varying soaking time and light exposure time on the mean Brillouin modulus of the anterior, mid-, and posterior cornea. ** $P<0.01$; *** $P<0.005$.

\section{Effect of pre-soaking time and UV light exposure time}

The dependence of the modulus change on two main parameters of CXL procedure, the photosensitizer pre-soaking time and the total optical energy was evaluated. Figure 6.2 depicts the comparison of Brillouin modulus in the anterior, middle and posterior region in four different conditions: a) untreated, b) 30 minutes presoaking with 5 minutes UV light exposure, c) 5 minutes presoaking with 30 minutes UV light, and 
d) 30 minutes presoaking with 30 minutes UV light exposure (standard Dresden protocol). From the Brillouin depth profiles and the average refractive index of 1.376 for corneal tissues, we measured the corneal thickness to be $0.98 \pm 0.035 \mathrm{~mm}$ for untreated controls, $0.88 \pm 0.05 \mathrm{~mm}$ for 30-min preasoak/5-min light, $0.87 \pm 0.03 \mathrm{~mm}$ for 5 -min presoak/30min light, and $0.84 \pm 0.03 \mathrm{~mm}$ for crosslinked corneas. All treatments induce statistically significant stiffening compared to the control in the anterior and mid-stroma regions (unpaired t-test, $p<0.01$ ), whereas no significant changes occur in the posterior region. However, significant differences (unpaired t-test, $p<0.01$ ) were measured between the soaking times of 5 and 30 minutes and between two illumination times of 5 and 30 minutes. At a soaking time of 5 minutes, the stiffness increase was reduced by $35-40 \%$ compared to standard Dresden protocol (i.e., CSI $=60-65)$. On the other hand, reducing light illumination to 5 minutes reduced mechanical efficacy by $65-70 \%$ (i.e. CSI $=35$ ). Figure 6.3 studies the dependence of CXL mechanical efficacy on the light exposure time by showing the corneal modulus of the anterior, central and posterior regions for UV light exposure time varying from 0 to 30 minutes with a fixed 30-min pre-soaking time. As shown in Figure 6.3 a, the posterior region on the cornea was not stiffened at any light exposure. In the midstromal region, only the full 30 minutes of light exposure produced a significant increase in modulus (unpaired t-test, $p<0.01$ ). And, in the anterior portion of the cornea a clear dosedependence of corneal stiffening to light exposure was observed.

Figure $6.3 \mathrm{~b}$ shows a highly significant linear dependence of the relative increase in anterior modulus on the light exposure time $(R 2>$ 0.98). The pre-soaking alone (without light) induced significant stiffening (unpaired t-test, $p<0.01$ ), as a result of corneal dehydration induced by the dextran-based solution. The mechanical effect of dehydration is further discussed in the next sections. 
Chapter 6. Brillouin microscopy of collagen cross-linking: non-contact depth-dependent analysis of corneal elastic modulus

a

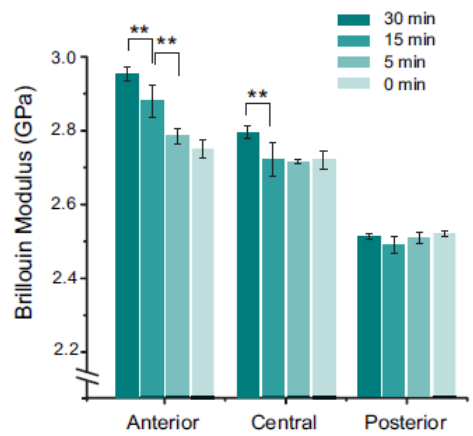

b

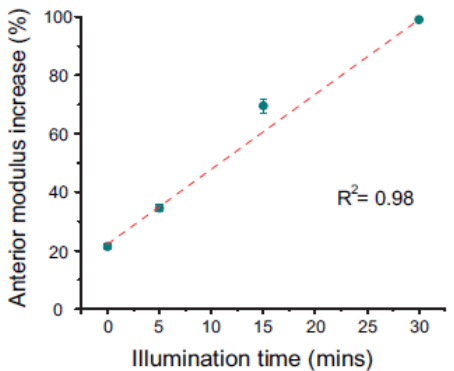

Figure 6.3: Mechanical outcome dependence on the light exposure time. (a) Mean Brillouin modulus of the anterior, mid, and posterior regions in the corneas treated with a presoaking time of 30 minutes and various UV exposure times of $0,5,15$ and 30 minutes, respectively. $* * P<$ $0.01 ; * * * P<0.005$. (b) The increase of mean Brillouin modulus in the anterior region as a function of exposure time. Circles, data; Error bars, standard deviations; Line, linear curve fit;

\section{Corneal collagen cross-linking without epithelial debride- ment}

Figure 6.4 shows the results on the mechanical efficacy of a transepithelial (ëpi-on) modality[106] evaluated by Brillouin microscopy. The Brillouin cross-sectional images and depth-profiles of a control (epi-on, 30 min presoak, no light) vs. a CXL treated cornea (epi-on, 30 min pre-soak, 30 min light) showed that the transepithelial CXL protocol was indeed capable of inducing corneal stiffening (unpaired ttest, $p<0.01$ ). However, the stiffening effect was lower than the one produced by the standard CXL protocol and was mostly confined to the anterior portion of the cornea, as shown in Figure $6.4 \mathrm{~d}$. From the Brillouin depth profiles, the corneal thickness was estimated to be $1.12 \pm 0.02 \mathrm{~mm}$ for controls and 1.05 $\pm 0.02 \mathrm{~mm}$ for crosslinked corneas. To estimate the overall efficacy of transepithelial CXL, CSI was calculated by averaging the modulus over 

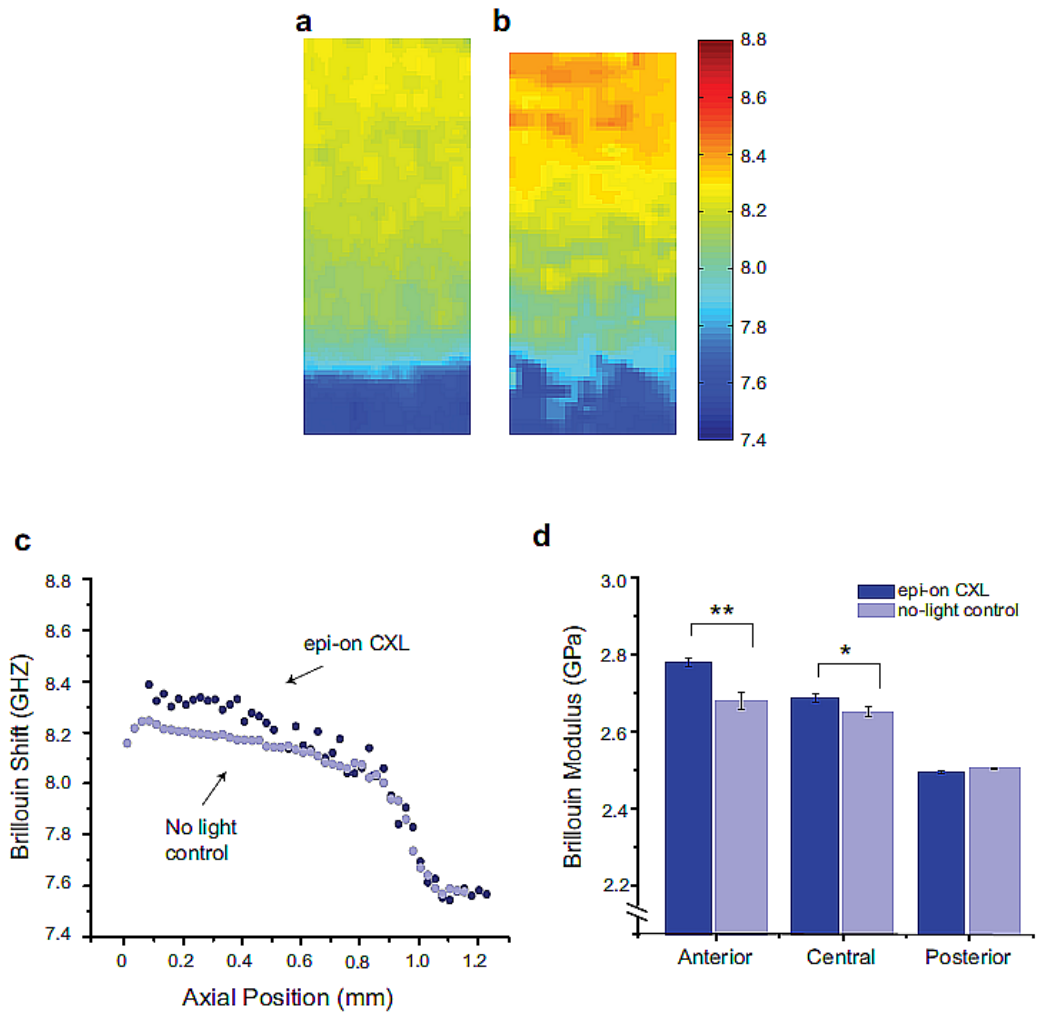

Figure 6.4: Brillouin mechanical characterization of transepithelial "epi-on" CXL. (a) A representative cross-sectional Brillouin image of normal porcine cornea. (b) A Brillouin image of the cornea after epi-on CXL. The horizontal and vertical span is $0.05 \mathrm{~mm}(x)$ by $0.8 \mathrm{~mm}(z)$ in $a$ and b. (c) Brillouin depth profile of epi-on CXL vs. soaked but not illuminated control cornea. (d) Mean Brillouin modulus of the anterior, mid-, and posterior regions for epi-on crosslinked $(N=2)$ vs. untreated corneas $(N=2)$. * $P<0.05 ; * * * P<0.005$. 
Chapter 6. Brillouin microscopy of collagen cross-linking: non-contact depth-dependent analysis of corneal elastic modulus

the entire corneal depth and comparing it to standard CXL. From the Brillouin measurement, the epi-on CXL was estimated to induce about $33 \%$ of the stiffening of epi-off CXL (i.e. CSI $=33$ ). For comparison, the mechanical stiffening of the two procedures was tested with goldstandard quasi-static rheology. This measurement showed that epi-on CXL induced stiffening of approximately $39 \%$ (i.e. CSI $=39$ ), consistent with the Brillouin-based measurement. As the experiments were performed ex-vivo immediately after the CXL procedure, this estimation includes the mechanical effect of the notably different hydration states of the cornea in the epi-on vs. epi-off procedure.
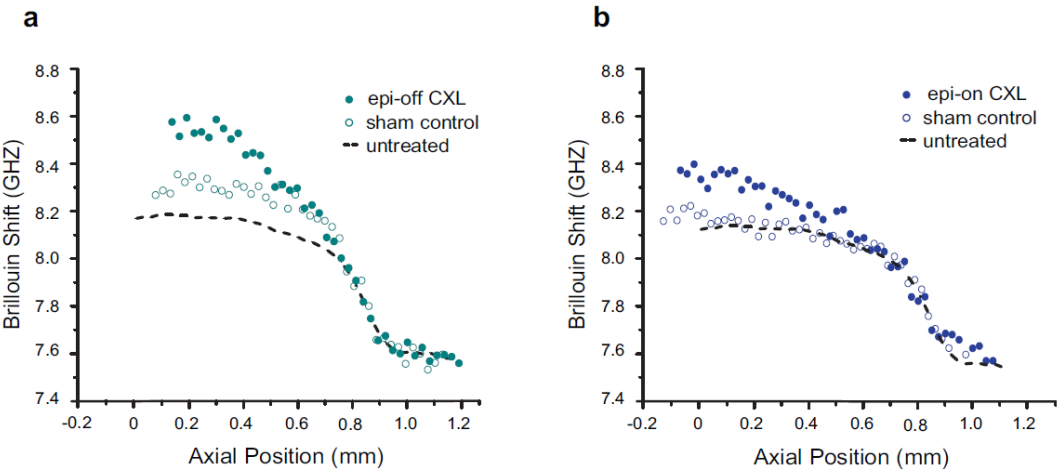

Figure 6.5: Mechanical effect of the corneal hydration state. (a) Brillouin depth profile of epi-off CXL vs. sham control (non-illuminated area of the same cornea). Dashed line indicates the average profile of untreated controls. (b) Brillouin depth profile of epi-on CXL vs. sham control. Dashed line indicates the average profile of untreated controls.

\section{Sham controls}

Sham control experiments were performed to evaluate the effects of different parameters (e.g. timing, soaking, evaporation) within the CXL procedure. UV light was administered to only a half of the eye after riboflavin soaking, whereas the other half of the eye was blocked during 
UV illumination. Figure $6.5 \mathrm{a}$ and $\mathrm{b}$ show the results for the epi-off protocol and the epi-on protocol, respectively. The Brillouin profiles of the UV-illuminated region and un-illuminated (sham) region of the eye are shown, along with the average Brillouin profile of untreated controls prior to soaking. As expected, the epi-off CXL profile in Figure 6.5 a and the epion CXL profile in Figure $6.5 \mathrm{~b}$ are consistent with the ones in Figure $6.1 \mathrm{c}$ and Figure $6.4 \mathrm{c}$, respectively. Importantly, the Brillouin moduli for the sham regions in Figure 6.5 a and Figure $6.5 \mathrm{~b}$ are equivalent to the results obtained with the controls used in Figures 6.1, 6.2,6.3 and 6.4, respectively. These control data were obtained with different storage times (up to $60 \mathrm{~min}$ ) of the samples and varying waiting times (up to $30 \mathrm{~min}$ ) between treatment and measurements. We concluded that within the short term of our experiments, differences in storage time or exposure to air had negligible effect on Brillouin modulus. On the other hand, the hydration and dehydration effects due to soaking solutions had significant mechanical effects, as previously noted (Figure 6.3). These effects are described in more detail below.

\section{Hydration effects on Brillouin modulus and shear modu- lus}

As expected, the epi-off dextran-based soaking caused the cornea to dehydrate (thickness $=0.90 \pm 0.03 \mathrm{~mm}$ ) while the epi-on saline-based soaking induced corneal swelling (thickness $=1.12 \pm 0.02 \mathrm{~mm}$ ). Figure 6.5 indicates that corneal dehydration is associated with an increased Brillouin modulus, whereas corneal hydration decreases Brillouin modulus. The dehydration caused by the application of dextran solution alone caused significant corneal stiffening with a CSI of about 20 . To validate this observation, gold-standard bulk rheology was used to compare shear modulus in samples soaked in dextran-based and saline-based solutions. As shown in Figure 6.6, corneas soaked with dextran-based epi-off solution had significantly higher shear moduli than corneas soaked with saline-based epi-on solution. This result is consistent with our Brillouin 
Chapter 6. Brillouin microscopy of collagen cross-linking: non-contact depth-dependent analysis of corneal elastic modulus

measurements.

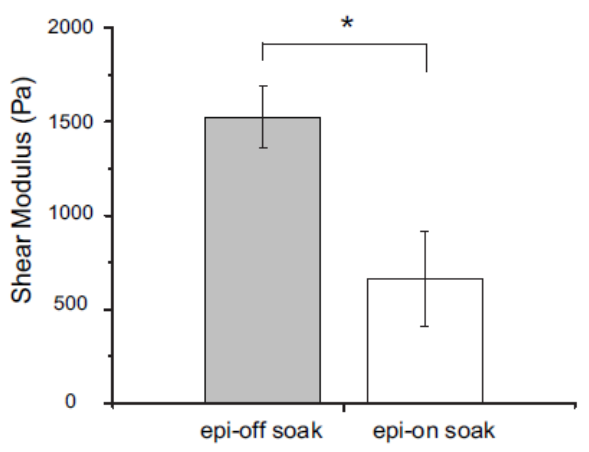

Figure 6.6: Shear modulus at $0.2 \mathrm{~Hz}$ of central corneal button (4 mm diameter). Buttons were resected and immersed in epi-off (dextran-based) solution $(N=6) v$ s. epi-on (saline-based) soaking $(N=6)$ for 30 min before measurement. Error bars: S.E.M. ${ }^{*} P<0.05$.

\section{Discussion}

Most CXL procedures tested around the world follow the original protocol described by Wollensak et al., which involves epithelial debridement, 30 min soaking in Riboflavin-Dextran solution and a 30 min application of UV light[226]. However, the significant thinning induced by the Riboflavin-Dextran solution during the procedure and the fear of endothelial cell damage make standard CXL only applicable to thicker corneas $(>350 \mu \mathrm{m})$. Moreover, the requirement of epithelial debridement to enable riboflavin diffusion into the corneal stroma results in significant eye pain in the first few days of post-operative recovery and increased risk of infections[204]. For these reasons, a significant effort has been undertaken to develop alternative procedures that result in reduced thinning, phototoxicity and recovery time while obtaining similar mechanical outcomes. In this context, transepithelial CXL[224, 232], custom epithelial debridement[120] and hypoosmolar photosensitizer 
Table 6.1: Mechanical efficacy of various CXL procedure quantified by the defined Corneal Stiffening Index. $R f=$ RiboflavinDextran; $B A C=$ benzalkonium chloride.

\begin{tabular}{l|c} 
& \\
CXL Procedure & Corneal Stiffening Index \\
\hline Untreated & 0 \\
\hline Epi-on $30 \mathrm{~min} \mathrm{BAC}+30 \mathrm{~min}$ light & 33 \\
\hline Epi-off $30 \mathrm{~min} \mathrm{Rf}+5 \mathrm{~min}$ light & 37 \\
\hline Epi-off $30 \mathrm{~min} \mathrm{Rf}+15 \mathrm{~min}$ light & 58 \\
\hline Epi-off $5 \mathrm{~min} \mathrm{Rf}+30 \mathrm{~min}$ light & 65 \\
\hline Epi-off $30 \mathrm{~min} \mathrm{Rf}+30 \mathrm{~min}$ light & 100 \\
\hline
\end{tabular}

solution[80] have been developed. However, the lack of a technology to monitor the mechanical outcome of various protocols has compromised the development of optimized CXL protocols. In this study, novel Brillouin microscopy was used to perform high-resolution noncontact and non-invasive mechanical characterization of corneas from intact eyes before and after CXL ex vivo in several settings relevant for application in the clinic. Brillouin microscopy enabled us to quantitatively determine the mechanical efficacy of various CXL protocols. We have introduced a universal metric to quantify the stiffening due to a certain CXL procedure relative to standard Dresden protocol. Table 6.1 summarizes the Corneal Stiffening Index (CSI) measured using Brillouin microscopy.

From the Brillouin elasticity images, it was found that corneal stiffening occurs in a depth dependent manner with most of the mechanical changes concentrated in the anterior portion of the corneal stroma. This result can be understood by considering the gradient of riboflavin diffusion along depth and the diminished light energy delivered to deep layers of the cornea due to absorption of the riboflavin in the anterior cornea $[23,193,197]$. Similar depth-dependence of corneal stiffening has been observed previously in a destructive mechanical test by Kohlhaas et al [113], who physically sectioned corneas at three different depths and 
Chapter 6. Brillouin microscopy of collagen cross-linking: non-contact depth-dependent analysis of corneal elastic modulus

observed a significant increase in the modulus of the anterior portion of the stroma but no increase in the mid-stromal region. In the present study Brillouin microscopy revealed a noticeable increase of the corneal modulus also in the central portion of the stroma. This result is consistent with the recent experimental and modeling studies that predicted a larger increase in the anterior stroma than Kohlhaas et al. and a significant increase of modulus also in the mid-stroma section [182]. It would be interesting to test if these results can be reproduced with other advanced mechanical measurement techniques $[107,109]$. The results of this study provide an insight into how the pre-soaking time and light dose affect the corneal mechanical outcome. A reduction of the pre-soaking time from 30 to 5 minutes reduced the effectiveness of cross-linking protocols by about $30 \%$ in terms of the increase of the corneal stiffness. The light exposure time was found to be a much more significant factor. A clear linear dependence was measured between the relative increase in anterior corneal modulus and UV light exposure time. This result is consistent with recent studies of Riboflavin UV cross-linking, which showed a linear dependence of the relative stiffness increase to total amount of UV energy absorbed [182]. In the central portion of the cornea, only the full 30 minutes of light illumination introduced a statistically significant increase in corneal modulus. In the study of the transepithelial CXL protocol, a clear depth-dependent stiffening was observed within the cornea with a statistically significant increase of corneal modulus in anterior and central regions of the stroma. The epi-on procedure was found to yield a CSI of 33, i.e. about one third of the mechanical efficacy of the standard CXL protocol. This estimation was validated with bulk rheology, which measured a CSI of 39, a reasonable agreement with the Brillouin measurement. The significant reduction in the mechanical efficacy of the epi-on procedure is probably the main factor responsible for the inferior clinical outcome demonstrated by recent clinical studies [115]. A prior study [224] that attempted to quantify the mechanical efficacy of transepithelial CXL found epi-on CXL to be approximately one 
fifth as efficient as the standard CXL (i.e., CSI $=20$ ).

The increased mechanical efficacy of this study is attributed to the improved protocol used for photosensitizer diffusion, developed by Raiskup et al.[164]. It should be noted that the epi-on CXL protocol may be less effective for the porcine corneas than human corneas because porcine corneas have thicker epithelium than human corneas [106]. Finally, the hydration state of the cornea was found to be relevant when assessing the mechanical efficacy as it contributes significantly to the mechanical properties of the cornea. This effect is particularly important for CXL procedures because the mechanical change due to corneal hydration is for the most part a transient effect and patients are expected to restore their normal hydration state in the long term [91, 119]. In this investigation, dehydration was measured to increase the corneal modulus whereas hydration decreased corneal modulus, which is in agreement with previous studies on corneas and other soft biological tissues [99, 143]. This effect was measured with Brillouin microscopy and confirmed with bulk rheology, as shown in Figure 6.6. The effect of hydration is also relevant to the long-term mechanical efficacy of the epi-on procedure because the soaking solution of epi-on CXL is based on PBS and thus tends to hydrate the cornea, whereas the epi-off procedure uses dextran that dehydrates the cornea and results in a CSI as high as 20.

In conclusion, this study has demonstrated that Brillouin microscopy is an accurate and useful tool to monitor and to determine the mechanical outcome of CXL in intact eyes at high spatial resolution. The dependence of the mechanical outcome on several operational parameters with and without epithelial debridement has been characterized and quantified in terms of a universal metric, CSI. Given the importance of the mechanical effect of hydration state and the transient nature of corneal hydration/dehydration due to CXL procedure, this study suggests that judging the long-term mechanical efficacy of CXL may require in vivo estimations. As Brillouin microscopy has been recently demonstrated in vivo[180], this technology may prove useful not only in the preclinical 
Chapter 6. Brillouin microscopy of collagen cross-linking: non-contact depth-dependent analysis of corneal elastic modulus

setting to develop new cross-linking agents and optimize CXL protocols, but also in the clinic as a monitoring tool to rapidly evaluate and longitudinally follow the mechanical outcome of the treatment. 


\section{7}

\section{Determination of corneal}

\section{biomechanical properties from deformation following an air-puff}

In the previous chapter we used a non-contact technique to measure the corneal elasticity map. In this study we use a different non-contact device, an air-puff tonometer and combine it with a sOCT in order to record the two-dimensional corneal deformation profile (temporal and spatial) during the air-puff event and to show differences between normal and cross-linked corneas.

This chapter is based on the publication by C. Dorronsoro, D. Pascual, P. Perez, S. Kling and S. Marcos, entitled "Dynamic OCT measurement of corneal deformation by an air puff in normal and cross-linked corneas" in 
Chapter 7. Determination of corneal biomechanical properties from deformation following an air-puff

Biomedical Optics Express.

The author of this thesis (i) did the sample preparation, (ii) performed the measurements in collaboration with C. Dorronsoro and P. Perez, (iii) did the data analysis in collaboration with C. Dorronsoro and (iv) revised the manuscript. 


\section{Introduction}

The mechanical properties of the cornea and its constituent materials are essential to relate its geometrical (and therefore optical) properties with its mechanical behavior $[6,50]$. Several corneal diseases, such as keratoconus [163], which lead to corneal deformation and thinning, affect dramatically the mechanical behavior of the cornea, and several treatments for these diseases attempt at modulating its biomechanical response. For example, an emerging treatment for keratoconus is the UV-Riboflavin cross-linking procedure, which aims at stiffening the weakened keratoconic corneas by creating intra and extrafibrillar bonds in the stromal collagen [195, 225, 226]. Other treatments such as implantation of intra-stromal rings aim at creating a supporting structure of the cornea, producing a flattening and a symmetrization of the anterior corneal surface [37]. This treatment may be highly dependent on the biomechanical properties of the cornea. Other corneal treatments largely rely on the biomechanical corneal response to produce (more or less permanent) refractive or corneal shape changes. For example, overnight orthokeratology uses reversed geometry contact lenses to reshape the cornea to correct low amounts of refractive errors [142]. Incisional refractive surgery [34], now of limited use because of its low predictability, relied completely on the biomechanical properties of the cornea. Corneal biomechanics is claimed to play a role (in combination with the ablation algorithm and laser-corneal tissue interactions) in the achieved corneal shape following laser refractive surgery, and particularly the corneal ectasia (corneal protrusion) developed post-LASIK by some patients [14, 42, 168]. Emergent corneal procedures for presbyopia (i.e. femtosecond Intracor treatment $[92,101]$ ) aiming at producing a change in corneal curvature in the central cornea, while maintaining its integrity, rely entirely on the biomechanical properties of the treated corneas. Despite the great need for the understanding and availability of corneal biomechanical parameters at the individual level, most of 
Chapter 7. Determination of corneal biomechanical properties from deformation following an air-puff

the data available today come from in vitro measurements of corneal biomechanics $[5,55,56,86,90,101,109,200]$. in vitro measurements have proved to be largely dependent on the technique used (with orders of magnitude differences in the reported corneal Young modulus of elasticity) and the experimental conditions (time post-mortem, hydration conditions, storage solutions, etc.). Although large efforts have been directed to the development of new paradigms for the analysis of experimental inflation data, in combination with more sophisticated models (corneal finite element models or corneal Micro-structure constituent based models $[53,56,109])$ the practical use of corneal biomechanical models have been somewhat limited by the reliability of the experimental input data and their validation. With no doubt, the understanding of corneal biomechanical properties, and the practical use of corneal biomechanical models will largely benefit from in vivo measurements. Many in vitro measurements require corneal excision and suppression of natural boundaries [56, 90, 101], and all methods suffer inevitably from unnatural corneal hydration states [86, 108]. Also, customized knowledge of the biomechanical properties at the individual level would allow incorporation of custom data into corneal biomechanical models and, in consequence, a better predictability of the outcomes. Knowledge of the corneal biomechanical properties at the individual level would allow, for example, a better selection of LASIK surgery candidates (identifying potential patients at risk of corneal ectasia), customization of intrastromal ring segments, deeper understanding of corneal biomechanics-modulating treatments or diagnosis and prognosis of keratoconus. The most important attempt to date to provide a clinical instrument to monitor the biomechanical response of the cornea has been the Ocular Response Analyzer (ORA, Reichert Ophthalmic Instruments, Buffalo, New York, USA) [125]. This instrument, an evolution of an air-puff tonometer, measures the changes in the light intensity recorded on a detector that collects light reflected from the cornea during the applanation produced by an air-puff impinging the cornea. The instrument derives values of the inward and 
outward pressure obtained during the dynamic applanation, which as a result of the viscous damping of the cornea, produces a delayed response. This difference is referred by the manufacturer as corneal hysteresis, due to its (indirect) relationship with corneal properties. Reports in the literature raise questions on the sensitivity of the technique to monitor changes in the biomechanical properties of the cornea. For example, there is conflicting evidence that the ORA is effective in identifying keratoconic patients [67, 125] or in detecting changes with cross-linking [230]. In general, the Ocular Response Analyzer does not provide a direct measure of corneal deformation upon applanation, nor a direct measurement of standard biomechanical parameters that describe the mechanical behavior of a material. Other approaches that have been suggested include Placido-ring based topography with applanation produced by a cantilever [76], optical coherence elastography [68] and ultrasonic spectroscopy [85]. Very recently, new approaches have been presented that attempt to overcome the lack of more direct measurements of corneal deformation in vivo, although the techniques are still at early stages. Oculus has recently presented a new high-speed Scheimpflug camera capable of imaging dynamic corneal deformation upon applanation with an air-puff. The instrument has not been yet widely released clinically, and the few available reports remain mainly qualitative [4] [C.J. Roberts et al, IOVS 2011, ARVO E-abstract 4384]. Scheimpflug imaging suffers from severe geometrical distortion and by optical distortions (in the posterior corneal surface) which need to be corrected before extracting quantitative information $[48,155,169]$. An alternative to anterior segment imaging in the eye to the Scheimpflug camera is optical coherence tomography (OCT) $[98,202]$. OCT has larger axial (and potentially lateral) resolution than Scheimpflug imaging, and high-speed OCT allows acquisitions of more than 100.000 A-scans/s [75, 77]. The combination of OCT technology with air-puff applanation has been recently published [3]. Despite the high-speed capabilities of the OCT system employed, the data obtained with their collinear configuration are limited to the dynamic acquisition 
Chapter 7. Determination of corneal biomechanical properties from deformation following an air-puff

of A-scans at the corneal apex, although in principle the technique could be applied on a larger corneal region (of primary interest for understanding full corneal dynamics). In this work, we present a new development of spectral OCT (sOCT) technology combined with air-puff applanation which allows monitoring corneal deformation over full corneal crosssections, besides high speed imaging of the corneal apex. We demonstrate the potential of the technique in vitro on porcine corneas, as well as in vivo in humans. In particular, we show the sensitivity of the technique to detect differences in corneal deformation between virgin corneas, corneas treated with Riboflavin + Dextran, and corneas after a UV-cross-linking procedure. These measurements are a first step for the estimation of corneal constituent material properties in vivo.

\section{Protocol}

The experiments were performed using an SOCT system in combination with a commercial tonometer (see chapter 2). All in vitro measurements were performed under constant IOP pressure $(18 \pm 1 \mathrm{~mm} \mathrm{Hg})$. This was achieved by means of an automated custom pressure control system (see chapter 2). The metrics described in chapter 2 were used to analyze the corneal deformation.

\section{Calibration and validation}

Three calibration and validation measurements were performed to detect potential artifacts in the images due to mirror movements or misalignments: (1) Dynamic imaging of the position of the mirror hole/tube tip during the air puff; (2) Measurement of a flat surface and rubber tonometry-probe eyes; (3) Repeated extended measurements of an in vitro eye. The potential effect of mirror vibrations was evaluated with measurements on rigid flat surfaces. Correct alignment was adjusted and validated when the images of a flat surface appeared un-distorted, and the deformation of the probe eye symmetrical. High measurement 
repeatability was obtained during 3 hours (every $10 \mathrm{~min}$ ). Increased variability after 3 hours of measurements was attributed to changes in the hydration properties of the eyes.

\section{Eyes and measurements}

Measurements were obtained on one eye of a 39-year old normal subject in vivo, and on fresh porcine enucleated eyes (virgin and after treatment) in vitro. Both in vivo and in vitro measurements were performed under similar instrumental conditions. Each measurement was repeated 5 times. The dynamic 2-D B-scan measurement mode (along the horizontal meridian) provides insights on the relative alignment of the eye with the air puff and the measuring instrument (sOCT), and therefore it was routinely used to obtain an optimal alignment before the 1-D measurements at full temporal resolution on the corneal apex. In measurements in vivo, the subject fixated a stimulus in the sOCT instrument. The subject was stabilized using a chin-rest. The study was approved by Institutional Review Boards and followed the tenets of the Declaration of Helsinki. The subject signed a consent form and was aware of the nature of the study. In measurements in vitro a custom eye holder allowed alignment of the eye to the instrument as well as implementing the IOP control system. The porcine eyes were obtained from a local slaughterhouse. A total of 6 eyes were used in the study. All eyes were initially measured untreated. The corneas were then de-epithelialized. Subsequent measurements were conducted after de-epitheliazation only (1 eye), after de-epitheliazation and instillation of Riboflavin $0.125 \%$ + Dextran 20\% solution during 30 minutes every 5 minutes (4 eyes), and after the full standard UV-crosslinking procedure with $3 \mathrm{~mW} / \mathrm{cm} 2$ 365-nm UV-A radiation, as described in [109] (2 eyes). All in vitro measurements were performed between 2 and within 6 hours post-mortem. Between measurements the eyes were kept in a refrigerator at $4^{\circ} \mathrm{C}$ in a chamber with cotton soaked in physiological saline (sodium chloride $0.9 \%$ ). 
Chapter 7. Determination of corneal biomechanical properties from deformation following an air-puff

\section{Results}
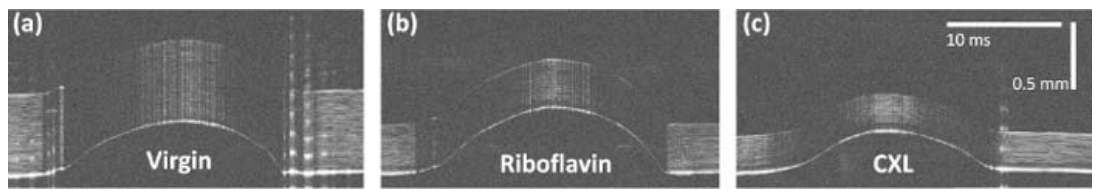

Figure 7.1: Examples of dynamic A-scan measurements on the same porcine eye in vitro, (a) before and (b) after treatments of Riboflavin and (c) UV-cross-linking (c). The vertical axis represents axial depth (with the anterior corneal surface down) and the horizontal axis represents time.
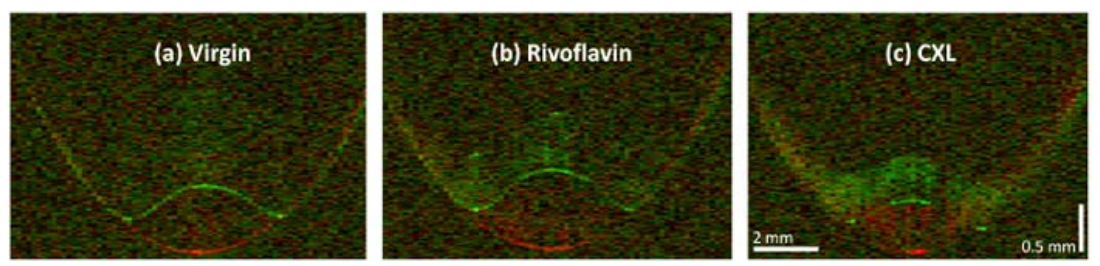

Figure 7.2: Horizontal sections of the cornea (B-scans), for three different conditions of the same in vitro porcine eye: (a) virgin eye with no treatment; (b) after instillation of Riboflavin; and (c) after UV-cross-linking. The stable non-deformed cornea (reference condition) is shown in red, and the deformed cornea is overlapped in green.

Figure7.1 shows three examples of dynamic A-scan measurements at the corneal apex on the same in vitro porcine eye before (Figure7.1a) and after treatments with Riboflavin (Figure7.1b) and UV-cross-linking (Figure7.1c). Figure7.2 shows B-scan measurements of the same cornea. Several images are captured during the corneal deformation event. The two extreme deformation conditions shown in Figure7.2 were used to extract the quantitative deformation parameters: the image of the stable non-deformed cornea (reference condition), shown in red; and the image of the maximum transient deformed cornea, overlapped in green. Figures7.1 and Figure 7.2 show the capability of the proposed methodology to retrieve information of the dynamic response of the cornea, with 
sufficient temporal (A-scans) and spatial (B-scans) resolution to extract quantitative parameters that describe the corneal deformation as a result of a 20-ms air-puff. The air-puff (conventionally used in tonometry) produces a very relevant corneal deformation in all conditions, although differences in the temporal and spatial shape of the corneal deformation occur across conditions.

Deformation amplitude and corneal thickness Deformation time Effects of treatment in the same eye deformation diameter

\section{Discussion}

We have demonstrated a new technique for the non-invasive imaging of the dynamic response of the cornea to an air puff inducing deformation, based on high-speed sOCT. The non-collinear configuration has allowed both for the high-speed evaluation of the dynamics of the corneal apex [3], and the imaging of the spatial corneal deformation over corneal cross-sections [4] [C.J. Roberts et al, IOVS 2011, ARVO E-abstract 4384]. This new generation of imaging techniques in vivo will potentially provide biomechanical information in individual eyes, avoiding the limitations of prior in vivo techniques [67, 108, 125, 151] (which provided only indirect information) and of in vitro techniques such as extensiometry [90] (which alter the anatomical integrity of the eye) or corneal button/whole eye inflation models $[56,109]$ (which are subject to hydration and preservation issues). The sensitivity of the technique has proven to be higher than existing techniques, as differences in corneal deformation dynamics across species and treatments are clearly detected. Furthermore, the entire deformation process can be dynamically visualized and studied quantitatively, allowing direct extraction of deformation parameters. For untreated porcine eyes, the different hydration states are the main source of variability across eyes. In treated porcine eyes, dehydration reduces this effect, and individual differences across eyes in tissue properties appear. In human eyes, the reduced signal to noise 
Chapter 7. Determination of corneal biomechanical properties from deformation following an air-puff

ratio introduces some uncertainty in the image analysis, but it is very low compared with the variability introduced by eye movements and positioning errors (not present in in vitro eyes). Despite the lower thickness of the human cornea as compared to the porcine corneas, the lower deformation amplitude (as well as shorter deformation durations and reduced deformation speeds) found in the study is indicative of a higher stiffness of human corneal tissue, in agreement with previous comparative reports of human and porcine corneal biomechanical properties $[55,226]$. However, this result could be affected by differences in the geometry and intraocular pressure across the tested eyes. In porcine corneas in vitro, where intraocular pressure was kept constant across eyes and treatments, we found a significantly different corneal response across conditions: virgin corneas, corneas after de-epitheliazation, after instillation of Riboflavin, and after UV-induced cross-linking. The corneal response after the UV-cross-linking treatment (and, to a minor extent, after Riboflavin) is consistent with an increase in corneal stiffness, as it produces reduced deformation amplitudes, reduced displaced volumes, and reduced deformation speeds to the same air-puff stimulus. This result is consistent with other studies aiming at estimating biomechanical properties of the cornea, following different approaches and time scales $[109,225]$. This study also shows that Riboflavin and UV-cross-linking produce changes in the temporal symmetry of the deformation event (a displacement in the peak amplitude toward the start of the deformation). The corneal recovery period is longer, and is also slower, compared to virgin and de-epithelialized corneas. The increasing/decreasing speed ratio appears as a very sensitive parameter to differences in treatment: it is increased with de-epitheliazation, reduced to the virgin corneas values after Riboflavin instillation (although with slightly reduced absolute speeds), and strongly increased with UV-cross-linking (with the absolute speeds reduced much more), indicating that the speed reduction is more important in the recovery period. These temporal and speed changes between the periods of increasing deformation and recovery 
suggest marked changes in the viscoelasticity of the tissue. The different corneal responses (to the same deformation stimuli) obtained after treatments indicate that there are marked biomechanical changes induced on the cornea. These differences are revealed by geometrical changes at a macroscopic scale upon deformation, but are likely the result of changes in the microscopic structure of the cornea. The relative contribution of the corneal geometry (mainly changes in thickness, and to a lesser extent in curvature), intraocular pressure and material constituent properties to the effect remains an interesting question, where the experimental data can provide insights. De-epitheliazation alone, which only produces a small reduction in corneal thickness (10\%) and minimal changes in the stromal arrangement, produces some changes in speed deformation parameters (but not other parameters), indicating a minor contribution of the epithelium to corneal biomechanics. The fact that Riboflavin alone produces significant changes in corneal deformation (in comparison with virgin corneas) is highly consistent with recent reports both from inflation models [108] and non-linear microscopy observations of collagen packing in Riboflavin + Dextran treated corneas [23], indicating both geometrical and ultra-structural changes. The instillation of Riboflavin + Dextran, and the subsequent corneal dehydration, also results in a reduction of corneal thickness (by 48\%), which is maintained after UV-cross-linking. However, the deformation parameters are capable of discriminating significantly across treatments: the deformation amplitude and the deformation speeds are decreased with Riboflavin and UV-cross-linking (while they are increased with de-epitheliazation alone) and the temporal distribution is affected (while it was not with de-epitheliazation). The difference in behavior is more apparent if we consider that all the treated corneas (with Riboflavin, followed by UVcross-linking or not) have been previously de-epithelialized. The differences in the deformation patterns that change with corneal thickness, cannot explain the changes in the biomechanical response observed after Riboflavin and UV-cross-linking. Conversely, the small change observed in 
Chapter 7. Determination of corneal biomechanical properties from deformation following an air-puff

the amplitude of the corneal deformation after Riboflavin, which is very similar to that of virgin eyes despite the drastic decrease (almost 50\%) in thickness, cannot be explained by a thickness change alone. The strong reduction in deformation amplitude (26\%) between UV-cross-linking and Riboflavin treated corneas (with similar thickness), reveals the changes induced by UV-cross-linking at the microscopic level, and the stiffening effect of the UV-cross-linking treatment. Our results also show that the deformation diameter and the displaced volume are reduced with the treatments, which also change the radius of the deformed cornea. Corneal curvature (and differences across eyes and conditions) could play a role in corneal deformation. Although corneal steepening has been reported clinically during the first month following UV-cross-linking [44], and this was observed also 24-hours after treatment in an in vitro model [109], differences in corneal radius of curvature were negligible immediately following treatment. This was further confirmed in our study, by imaging horizontal sections of the non-deformed corneas at constant pressure. We have demonstrated a technique for the evaluation (in vivo and in vitro) of dynamic corneal deformation, capable of detecting differences across species and treatments. Tempora and spatial measurements provide complementary (or even coincident) descriptions of the deformation event. In particular, using this technique, we have confirmed non-invasively that UV-cross-linking induces changes in the corneal biomechanical properties, as recently reported using invasive techniques $[109,151]$. The differences must be the result of changes in constituent properties of the cornea, and not solely the results in changes in corneal thickness, geometry or IOP, as these were constant across some of the conditions. The retrieved deformation parameters (amplitude, radius, diameter and volume of the deformation, as well as duration and speeds of the deformation periods), in combination with measurements of corneal biometry and IOP, pulse calibration, will be extremely valuable experimental data in corneal biomechanical models. Although these findings are of interest in the understanding of tonometric 
measurements of the cornea, the ultimate goal is to obtain the biomechanical properties of the tissue based on image-based measurements of corneal deformation. This information will serve as experimental inputs in finite element models of the normal, pathological and treated cornea [108], at an individual level, to improve diagnosis and prognosis of the disease and treatment. The developed technology can also help to understand the sources of the variation in the deformation response across corneas and conditions, including biomechanical properties of the tissue (elasticity and viscoelasticity), the changes in the structural properties of the corneas (as geometry) or the effect of IOP pressure (which was kept constant in our experiment).

\section{Conclusions}

The combination of high-speed sOCT imaging with air puff deformation shows a great potential as a research and clinical tool to retrieve the biomechanical properties of individual corneas, in vivo. The non-collinear configuration presented in this study, makes available both, images of the corneal deformation over full corneal cross-sections, and also high speed measurements of the temporal evolution of the corneal apex. The quantitative description provided by the set of deformation parameters proposed has proven to be sufficiently sensitive to detect, not only differences across species and treatments, but also across individual eyes. The differences found must be the result of changes in constituent properties of the cornea, and not a consequence of changes in corneal thickness, geometry or IOP. These measurements could serve as potential inputs to be used in finite element models of the normal, pathological and treated cornea, and are a first step for the estimation of the biomechanical properties of corneal tissue, at an individual level and in vivo, to improve diagnosis and prognosis of diseases and treatments. 
Chapter 7. Determination of corneal biomechanical properties from deformation following an air-puff 


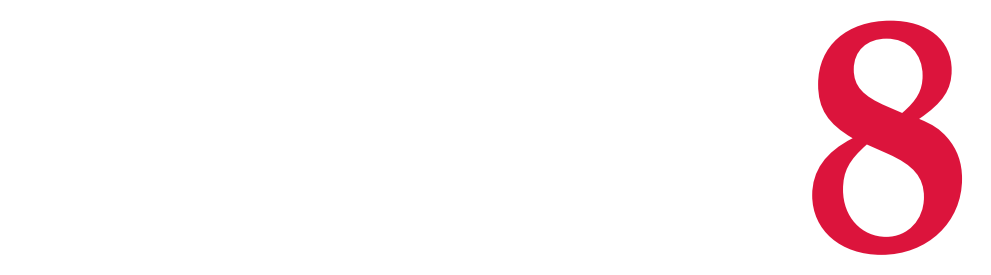

Contributing factors to corneal deformation in air-puff measurements

In the previous chapter we combined an air-puff with a sOCT. In this study we use a Scheimpflug based air-puff system, which recently has been FDA approved as a non-contact tonometer, to assess differences in the temporal and spatial corneal deformation under different conditions.

This chapter is based on the publication by S. Kling and S. Marcos, entitled "Contributing factors to corneal deformation in air-puff measurements" in Investigative Ophthalmology and Vision Science and was elected as research highlight. The work was also presented in an invited oral contribution at the 2012 8th International Congress of Corneal 
Chapter 8. Contributing factors to corneal deformation in air-puff measurements

Cross-linking in Geneva (Switzerland).

The author of this thesis (i) did the sample preparation, (ii) performed the experiments, (iii) did the data analysis and (iv) prepared the manuscript in collaboration with S. Marcos. 


\section{Introduction}

The use of an air-puff to produce a deformation of the cornea is frequently used in ophthalmology to measure the intraocular pressure (IOP). In applanation tonometry the IOP is inferred from the force required to flatten (applanate) a constant area of the cornea, making assumptions regarding the contribution of the corneal thickness on the measurements[29, 166]. Typically, in standard applanation tonometry, the force on the cornea is applied by a probe in contact with the cornea. In contrast, air puff non-contact tonometers[186] use a rapid air-pulse to flatten the cornea. Recently, air-puff systems have been proposed as potential tools to determine corneal biomechanical properties, taking advantage of the fact that corneal deformation depends on its thickness (from which tonometry measurements need to be corrected from) and the corneal biomechanical response. The Ocular Response Analyzer[125] uses an air-puff to deform the cornea into a slight concavity and monitors the pressures at which the cornea flattens inward and outward, correlating this difference with corneal hysteresis. The availability of high-speed imaging instruments has allowed to capture cross-sections of the cornea in real-time during an air puff event. Dorronsoro et al[45] used a high speed spectral OCT system in synchronization with an air-puff, and provided seven parameters (amplitude, diameter and volume of the maximum deformation, as well as duration and speed of the increasing deformation period and the recovery period) that described the dynamics of corneal deformation. An increase in corneal rigidity by cross-linking in pig corneas (under controlled IOP) resulted in a smaller corneal deformation by the air-puff. Recently, a commercial instrument based on Scheimpflug imaging[93] has been released (Corvis, Oculus, Wetzler, Germany), which captures the corneal dynamic deformation at a speed of about 4400 images/s and extracts the temporal apex deformation, its velocity and the applanation length from the temporal and spatial profiles of corneal deformation. The breadth of information extracted from the direct view of the corneal de- 
Chapter 8. Contributing factors to corneal deformation in air-puff measurements

formation holds promise to yield clinically relevant parameters correlated to the biomechanical properties. The possibility of measuring corneal stiffness in-vivo would allow an early diagnosis of certain pathologies (such as keratoconus, which weakens the corneal tissue[52, 150]), as well as the quantification of the success of treatments that alter the corneal stiffness (such as collagen cross-linking, increasingly used to stiffen the corneal tissue and to halt keratoconic progression[195]). Only few other non-destructive systems to measure the corneal biomechanical properties in-vivo have been proposed: Ultrasound techniques[206], with limited success due to the patients discomfort caused by eye immersion, and Brillouin microscopy[177], a new emerging non-contact technique which allows determining the corneal static elasticity modulus with high spatial resolution, although in its present form it cannot access the dynamic properties of the cornea. According to studies on corneal biomechanics,[17] the cornea is a viscoelastic tissue and hence, its relaxation time would be an interesting factor to be measured along with corneal elasticity. Measuring corneal deformation follwoing an air-puff therefore is a promising technique to measure the dynamic biomechanical properties of the cornea. However, corneal deformation also depends on other geometrical and structural factors besides the inherent mechanical properties of tissue. This is particularly relevant to interpret the in-vivo measurements provided by corneal air-puff instruments. In our study, we evaluated the contribution of different parameters to the corneal deformation by an air puff: The influence of IOP (by performing experiments in vitro at different IOPs), the role of corneal thickness and corneal dehydration (by comparing the deformation of virgin corneas to that of corneas treated with a dextran-containing solution), the role of corneal rigidity (by comparing the deformation of nontreated corneas with corneas treated with CXL), the role of corneal boundaries (absence/presence of the sclera, by comparing the deformation of corneal buttons attached to an artificial chamber and deformation of whole eyes), and the role of the ocular muscles and other in vivo/in vitro potential differences in 
tissue properties (by comparing the deformation in vitro and in vivo). The information provided by the experiments allowed us to identify the parameters that best relate to the corneal biomechanical properties.

\section{Conditions and Protocols}

The air-puff system based on Scheimpflug imaging (see chapter 2) was used for the experiments. Corneal deformation was imaged in pig and human corneas, in vitro and in vivo measurements under different conditions of pressure, dehydration, corneal stiffness and boundary (fixation) conditions. Measurement conditions and different data sets are summarized in Table 8.1.

Table 8.1: Overview on the different data sets, treatment and conditions used in the experiments. CCT = central corneal thickness.

\begin{tabular}{|c|c|c|c|c|}
\hline $\begin{array}{c}\text { data } \\
\text { set }\end{array}$ & treatment & $\begin{array}{l}\text { CCT } \\
(\mu m)\end{array}$ & $\begin{array}{c}\text { number } \\
\text { of eyes }\end{array}$ & condition \\
\hline \multirow{3}{*}{1} & virgin (pig) & 898 & \multirow{3}{*}{5} & \multirow{3}{*}{ in vitro } \\
\hline & Riboflavin-Dextran (pig) & 586 & & \\
\hline & cross-linking (pig) & 211 & & \\
\hline \multirow[b]{2}{*}{2} & globe virgin (pig) & 878 & \multirow{2}{*}{9} & \multirow{2}{*}{ in vitro } \\
\hline & button virgin (pig) & 993 & & \\
\hline \multirow{2}{*}{3} & \multirow{2}{*}{ virgin (human) } & 551 & 9 & in vivo \\
\hline & & 768 & 5 & in vitro \\
\hline
\end{tabular}

\section{Pig eyes}

A total of 14 fresh enucleated pig (six-month old) eyes were obtained from a local slaughterhouse (prior to scalding) and used within 4 hours post-mortem. 
Chapter 8. Contributing factors to corneal deformation in air-puff measurements

\section{Human eyes}

Donor eyes were obtained through an agreement between the Fundación Universidad Autónoma de Madrid and Instituto de Óptica (CSIC). Human patients were normal volunteers, and signed an informed consent after receiving an explanation regarding the nature of the study. All protocols followed the Tenets of the Declaration of Helsinki and had been approved by the Institutional Review Boards. in vivo air-puff measurements were performed on 9 eyes of 7 healthy human subjects aging ( 25 to 53 years). in vitro measurements were performed in 5 enucleated donor eyes (80 to 95 years).

\section{Different IOPs (data set 1)}

A set of 5 in vitro virgin porcine whole eye globes was used to assess the impact of IOP on the corneal deformation measurement. The eyes were mounted in a customized holder preventing global motion during the air-puff ejection. Then the IOP was increased from 15 to $45 \mathrm{mmHg}$ and 5 air-puff measurements were taken in each step. For the IOP adjustment an automated pressure system was used that has been described previously.[109] Briefly, a needle was introduced through the optical nerve head connecting the eye over a syringe to a water column. Custom routines written in Matlab (Mathworks, Natick, MA, USA) were then used to control a motor moving a syringe in order to change the height of the water column. Simultaneously, the pressure in the system (and hence the IOP) was recorded by a pressure sensor for accurate regulation.

\section{Corneal thickness and hydration state (data set 1)}

The eyes from (I) were subsequently used in an experiment to assess the impact of corneal thickness and hydration state, produced by RibloflavinDextran solution.[108] IOP was set to $15 \mathrm{mmHg}$. In this experiment, the first two stages of a standard UV collagen cross-linking treatment were performed: (i) De-epithelialization, (ii) Application of 0.125\%- 
Riboflavin-20\%-Dextran solution for 30 minutes This included removing the epithelium and applying Riboflavin-20\%Dextran solution . As in (I), the IOP was varied from 15 to $45 \mathrm{mmHg}$ and 5 air-puff measurements were taken in each IOP step.

\section{Corneal rigidity (data set 1 )}

The eyes from (II) were subsequently used to assess the impact on corneal rigidity, produced by a UV collagen cross-linking procedure.[109] The IOP was adjusted to $15 \mathrm{mmHg}$, The last stage of a cross-linking treatment was performed: (iii) illumination with $367 \mathrm{~nm}$ UV-light and $3 \mathrm{~mW} / \mathrm{cm} 2$ 30 minutes. As in (I) and (II) the IOP was varied from 15 to $45 \mathrm{mmHg}$ and 5 measurements were taken in each IOP step.

\section{Corneal buttons versus whole eyes (data set 2)}

A new set of 11 porcine eyes in vitro was used to compare corneal deformation in whole globes and corneal buttons, which allowed investigating the contribution of the sclera to the overall deformation. Whole eye globes were mounted in a customized holder preventing global motion during the air-puff ejection. The IOP was set to $15 \mathrm{mmHg}$ and 5 air-puff measurements were taken on each eye Then the corneas were excised and mounted in an artificial anterior chamber. The internal pressure was set to $15 \mathrm{mmHg}$ (same as the IOP before) and 5 air-puff measurements were on each cornea.

\section{in vitro versus in-vivo (data set 3 )}

in vitro experiments in 5 human donor eyes allowed comparing the deformation observed in human and porcine eyes. in vivo experiments were performed on 7 subjects with normal IOP. Comparison of corneal deformation in vivo and in vitro allowed us to determine the contribution of ocular muscles to the overall deformation, and of the potential physiological differences in vivo/in vitro in the investigations on enucleated 
Chapter 8. Contributing factors to corneal deformation in air-puff measurements

eyes. In- vitro human whole eyes were mounted in a customized holder (similar to the one used in porcine eyes, but scaled to fit human eye globe dimensions). IOP was set to $15 \mathrm{mmHg}$. 5 measurements were obtained in this condition per eye. in vivo measurements were obtained following typical clinical protocols. 1 measurement was obtained per eye.

\section{Analysis}

The corneal profile for each image captured during the deformation event was exported from the CORVIS software. Corneal apex indentation as a function of time and the corneal profile at maximal deformation were analyzed. The following parameters were obtained from this deformation data: (1) Peak to Peak distance (PDmax), i.e. the lateral distance between the two convex peaks in the corneal profile at maximal deformation (Figure 2.16A); (2) Temporal symmetry factor (TSym) that describes the degree of symmetry of the corneal apex versus time function (Figure 2.16B). Tsym is the ratio of the two areas under the curve separated by $T_{2}$ and can be calculated from:

$$
T_{\text {sym }}=\frac{\sum \Delta Y_{\text {apex }} T_{T_{1}}}{\sum \Delta Y_{\text {apex }} T_{3}}
$$

where $T_{1}$ is the time where the deformation starts, $T_{2}$ is the time at maximal deformation, and $T_{3}$ is the time when the direct deformation finishes. $\Delta Y_{\text {apex }}$ is the indentation of the corneal apex recorded by the Corvis system. $T_{s y m}$ indicates the degree of temporal symmetry in the deformation $\left(T_{s y m}=1\right.$ means a purely axis-symmetric temporal deformation). (3) Direct air-puff response, which describes the corneal deformation as direct response to the applied air-puff, i.e. as long as the air-puff applies a force onto the cornea (indicated by time points $T_{1}$ and $T_{3}$ ). (4) Indirect air-puff response was defined as the remaining deformation after $T_{3}$, i.e. after the elastic air-puff response.It depends on the viscoelastic properties and, for the in vivo condition, it depends 
additionally on the damping due to the ocular muscles. (5) Corneal central thickness before the air-puff deformation.

Statistical analysis was carried out using SPSS software (IBM). Multivariate analysis of variance (MANOVA) was performed to test the overall effect of IOP, geometry and treatment conditions on the corneal deformation. MANOVA with repeated measures was used to test the repeatability of the measurements. One-way analysis of variance (ANOVA) was performed to test the differences between individual groups. Confidence levels were set to $95 \%$.

\section{Results}

The major dependencies of the geometrical deformation parameters for the different evaluated corneal conditions are summarized in Table 8.2.

Table 8.2: Summary of experimental results.

\begin{tabular}{l|l|l|l} 
& $\begin{array}{l}\text { peak distance } \\
\text { (PD) }\end{array}$ & $\begin{array}{l}\text { maxiaml apex } \\
\text { indentation }\end{array}$ & $\begin{array}{l}\text { temporal } \\
\text { symmetry } \\
\text { factor }\left(T_{\text {sym }}\right)\end{array}$ \\
\hline IOP & $\begin{array}{l}\text { linear } \\
\text { dependency }\end{array}$ & $\begin{array}{l}\text { strong } \\
\text { non-linear } \\
\text { dependency }\end{array}$ & no effect \\
\hline Hydration / CCT & no effect & no effect & no effect \\
\hline rigidity & influence & influence & influence \\
\hline button / whole globe & no effect & no effect & influence \\
\hline in vitro / in vivo & no effect & no effect & no effect \\
\hline
\end{tabular}

\section{Effect of intraocular pressure (IOP) - data set 1}

Temporal apex deformation Figure 8.1A presents the apex deformation of a virgin porcine whole globe across time. The maximal indentation was very sensitive to variations in IOP $(p<0.001)$. In virgin eyes the maximal indentation varied from 1.262 to $0.550 \mathrm{~mm}$ for IOP ranging between 15 
Chapter 8. Contributing factors to corneal deformation in air-puff measurements

and $45 \mathrm{mmHg}$, decreasing at a rate of $-23.9 \mu \mathrm{m} / \mathrm{mmHg}$. The temporal symmetry factor $($ Tsym) did not change significantly $(\mathrm{p}=0.298)$ with IOP.
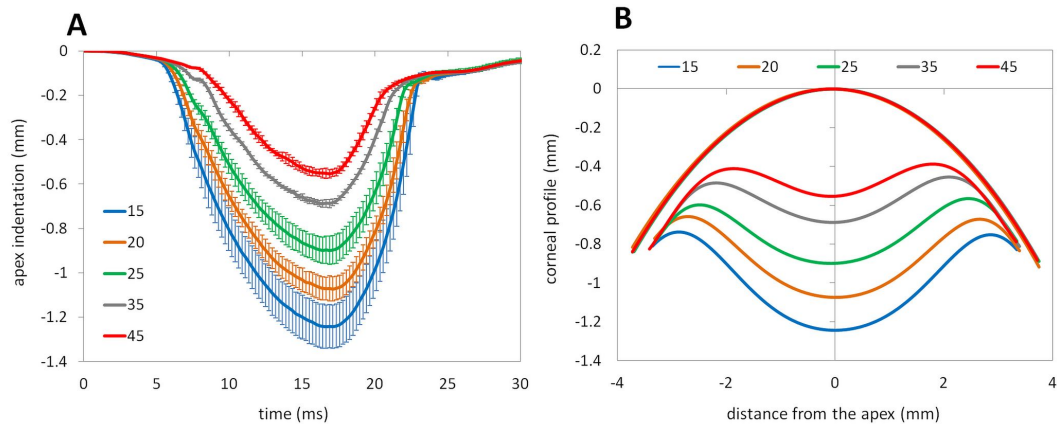

Figure 8.1: (A) Corneal apex deformation as a function of time (B) Corneal profile at maximal deformation. Different colors represent different IOPs, ranging from 15 to $45 \mathrm{mmHg}$. Data are for whole porcine eyes. Each point is the average of measurements in 5 eyes. Error bars stand for the standard deviation across eyes.

\section{Spatial deformation profile}

The corneal profile at maximal deformation is shown in Figure 8.1B. For a better comparison the initial apex position was shifted to zero. As expected (and similar to the maxiaml apex deformation) a strong dependency of the IOP on the maximal distance between peaks $\left(P D_{\max }\right)$ was found $(p<0.001)$.

\section{Effect of dehydration and CXL - data set 1}

\section{Temporal apex deformation}

Figure 8.2A shows the different temporal deformation patterns of the corneal apex throughout the CXL treatment. Riboflavin-Dextran instillation did not induce a significant $(\mathrm{p}=0.978)$ difference in the apex indentation with respect to virgin corneas (virgin: $1.26 \pm 0.16 \mathrm{~mm}$; Riboflavin-Dextran: $1.30 \pm 0.08 \mathrm{~mm}$ ). On the contrary, after CXL we 

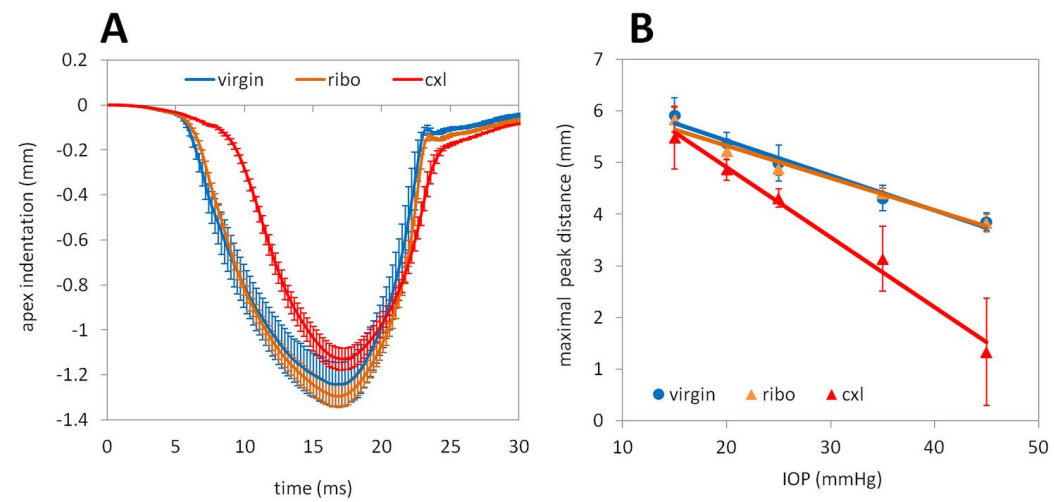

Figure 8.2: (A) Corneal apex deformation during the deformation event (IOP fixed at $15 \mathrm{~mm} \mathrm{Hg}$ ); (B) corneal profile peakto-peak at distance at maximum deformation (PDmax) as a function of IOP. Data are average across 5 whole globes each: virgin corneas (blue); Riboflavin-Dextran-treated corneas (yellow); cross-linked treated corneas (red). Each point is the average of measurements in five eyes.

observed a pronounced decrease of the apex indentation (by a factor of 1.67) and change (by factor 1.14) in the symmetry of the temporal apex deformation (Tsym), both compared to virgin. In general, in virgin corneas and in Ribofavin-Dextran treated corneas the inward deformation time took longer than the backward deformation (virgin corneas: Tsym $=1.653 \pm 0.25$; Riboflavin-Dextran treated corneas: Tsym $=1.463 \pm$ 0.21). This relation changed after CXL, where the durations of the inward and outward deformation were similar (Tsym $=1.002 \pm 0.16)$. Differences in the temporal symmetry between virgin and Riboflavin-Dextran corneas and differences in the temporal symmetry between virgin and cross-linked corneas were both highly significant $(p<0.001)$.

\section{Spatial deformation profile}

Figure $8.2 \mathrm{~B}$ shows the peak-to-peak distances of the corneal profile at maximal deformation as a function of IOP (profiles shown in Figure 8.1B for virgin porcine cornea), for virgin corneas, Riboflavin-Dextran treated 
Chapter 8. Contributing factors to corneal deformation in air-puff measurements

corneas, and cross-linked corneas, respectively. No significant difference in the peak distance was observed due to Riboflavin-Dextran instillation $(\mathrm{p}=0.891)$, while CXL produced a highly statistically significant decrease $(p<0.001)$.
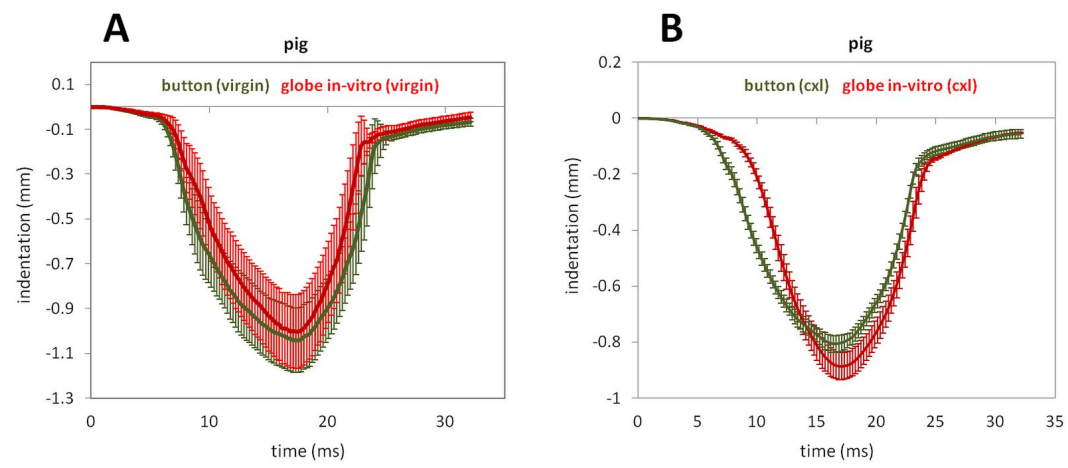

Figure 8.3: Corneal apex deformation across time in in vitro porcine corneas at IOP $=15 \mathrm{mmHg}$ (whole globes versus corneal buttons). Differences reveal the effect of the sclera. (A) Virgin porcine corneas: Each point is the average of measurements in 9 eyes. (B) Cross-linked porcine corneas: Each point is the average of measurements in 5 eyes.

\section{Difference between button and whole globe - data set 2}

\section{Temporal apex deformation}

Figure 8.3 shows the apex deformation of in vitro porcine corneas at normal IOP (15 mmHg). The maximal indentation depth of the apex (whole globes: $1.221 \pm 0.43 \mathrm{~mm}$; corneal buttons: $1.049 \pm 0.15 \mathrm{~mm}$ ) was not significantly $(\mathrm{p}=0.113)$ affected by the boundary condition. The deformation of the cornea in whole globes tended to be shorter in time, and the temporal symmetry was significantly $(\mathrm{p}=0.002)$ lower (whole globes: Tsym $=1.426 \pm 0.16$; corneal buttons: Tsym $=1.582 \pm 0.17$ ). The red curves (representing the in vitro porcine eye in Figure 8.3A and the in vitro human eye in Figure 8.4) show the same treatment condition and 
hence differences are due to the inter-species differences between pig and human corneal tissue. Figure 8.3B shows a comparison of air-puff corneal deformation in corneal buttons and whole globes following CXL. Similarly a shorter deformation was observed for whole globes compared to corneal buttons and temporal symmetry was significantly $(p<0.001)$ different (cross-linked whole globes: Tsym $=1.002 \pm 0.16$; cross-linked corneal buttons: Tsym $=1.309 \pm 0.132$ ).

\section{Spatial deformation profile}

Maximal peak distance was not significantly different $(\mathrm{p}=0.576)$ between whole globe $(P D m a x=5.57 \pm 0.98 \mathrm{~mm})$ and button $\left(P D_{\max }=5.74 \pm 0.39\right.$ $\mathrm{mm}$ ) porcine corneas, indicating a negligible contribution of the sclera in the spatial deformation profile.

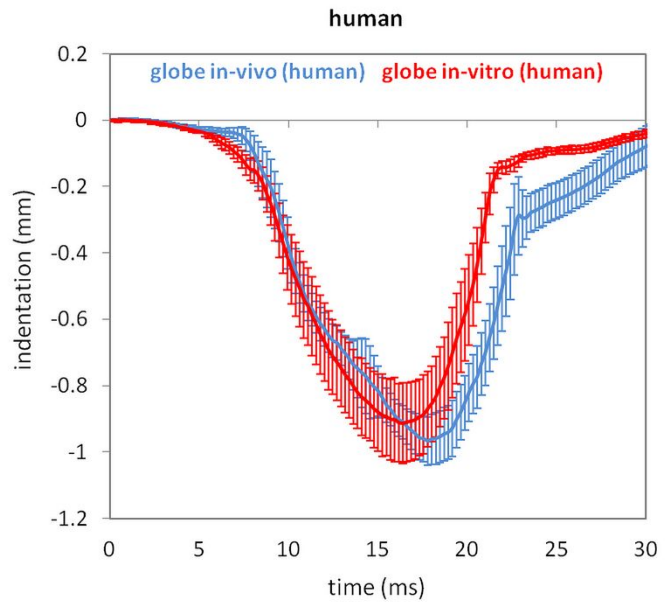

Figure 8.4: Corneal apex deformation across time in human globes at IOP $=15 \mathrm{mmHg}$ (in vitro versus in vivo). Each point is the average of measurements in nine eyes in vivo and five eyes in vitro, respectively. Differences reveal the effect of ocular muscles. 
Chapter 8. Contributing factors to corneal deformation in air-puff measurements

\section{Difference between in vitro and in-vivo - data set 3}

\section{Temporal apex deformation}

Figure 8.4 presents the apex deformation of human eyes at normal IOP $(15 \mathrm{mmHg})$. There is a trend for a larger deformation in the in-vivo than in vitro, although the differences are not statistically significant $(p=0.290)$. The trend could be associated to the age-differences between both groups. No significant difference $(\mathrm{p}=0.704)$ was found between the temporal symmetry in human whole globes in vitro $(\mathrm{Tsym}=1.600 \pm 0.212)$ and in vivo $($ Tsym $=1.608 \pm 0.264)$.

\section{Spatial deformation profile}

Maximal peak distance was not significantly different $(\mathrm{p}=0.384)$ between in vivo human whole globes (PDmax $=4.787 \pm 0.18 \mathrm{~mm}$ ) and in vitro human whole globes (PDmax $=4.778 \pm 0.33 \mathrm{~mm}$ ), indicating a negligible contribution of the ocular muscles in the spatial deformation profile.

\section{Central corneal thickness - all data sets}

Corneal thickness differed largely across the different treatment conditions and data sets (see 8.1). Riboflavin-Dextran treatment induced a decrease in corneal thickness by $65.3 \%$, which further decreased by $35.9 \%$ after CXL. in vitro virgin human corneas were on average 1.39 times thicker than $\mathrm{n}$ vivo human corneas. We also found that corneas in virgin whole globes were $115 \mu \mathrm{m}$ thinner than the contralateral corneas measured as corneal buttons.

\section{Repeatability of measurements - data set 2}

All four analyzed parameters were highly repeatable across the four consecutive measurements. Standard deviations of repeated measurements were: maximal apex indentation (whole globe: $\pm 0.064 \mathrm{~mm}$; corneal button: $\pm 0.027 \mathrm{~mm}$ ), maximal peak distance (whole globe: $\pm 0.516 \mathrm{~mm}$; 
corneal button: $\pm 0.077 \mathrm{~mm}$ ), Tsym (whole globe: \pm 0.102 ; button: \pm 0.045 ) and central corneal thickness (whole globe: $\pm 13.3 \mu \mathrm{m}$; corneal button: $\pm 15.4 \mu \mathrm{m})$.

\section{Discussion}

The investigation of the effect of different controlled conditions on the corneal deformation upon air-puff has allowed a better understanding of the different factors affecting corneal deformation, and a better interpretation of these measurements in human patients. The high repeatability of Corvis measurements reported in a recent study was confirmed by our measurements. [94] We found that corneal deformation following an air-puff is highly sensitive to IOP. The amount of apical deformation showed an almost linear correlation with IOP, which confirms the usefulness as a non-contact tonometer.[186] It also indicates the need for including IOP as parameter in the interpretation of the corneal deformation patterns measured in-vivo. In addition, we found that the maximal indentation depth of the apex was correlated with a stiffening of the corneal tissue due to CXL. The fact that Riboflavin-Dextran treated corneas experienced a similar deformation to virgin corneas suggests that corneal thinning and geometry changes cancel out the effect of potential biomechanical changes induced by Riboflavin-Dextran. Thinner corneas are generally expected to deform more, therefore an increase in corneal density (due to dehydration) could explain the increase in corneal rigidity with dehydration. It is know that dehydration can change the intrinsic biomechanical properties of the corneal tissue.[86] In our study we did not find a dependency between corneal deformation and central corneal thickness, likely because the observed thickness changes all related to density changes. A recent study [207] suggests that water intake into the cornea decreases its viscosity and cancels out the effect of thickness increase. Future studies might address the corneal response after partially removing corneal tissue (such as after LASIK or PRK surgery). The depen- 
Chapter 8. Contributing factors to corneal deformation in air-puff measurements

dency of the corneal deformation pattern on geometrical factors would be a limitation in the direct clinical application of air-puff deformation parameters to assess corneal biomechanical properties, as it implies the use of correction formulae in order to isolate the biomechanical contribution from other factors. We found that the temporal symmetry factor was highly sensitive to the CXL treatment, and depended only slightly on IOP and corneal thickness/dehydration. A change in temporal symmetry suggests an alteration of the viscoelastic material properties (such as the relaxation time). This change in the dynamic behavior of the cornea after CXL can be supported by a recent experimental study, where an uniaxial creep test in cross-linked corneal tissue suggested changes in the viscoelastic properties, in comparison with virgin corneal tissue (Richoz O., 2012, 8th CXL-Congress). Moreover, the delayed start of the apex indentation in cross-linked corneas (compared to virgin or RiboflavinDextran corneas) indicates a higher resistance to deformation due to an increase of corneal stiffness. In our experiments, the sensitivity of the maximal peak distance to CXL increased drastically with higher IOP (Figure 8.2B). This effect may be interesting for air-puff measurements in glaucoma patients. The corneal deformation pattern of a whole globe and corneal button showed significant differences in the temporal deformation pattern (Tsym), whereas no significant difference was observed in the maximal apex indentation and peak distance. As the sclera only affected the temporal apex deformation, the scleral deformation upon air-puff must be very small. A minor contribution to the differences between corneal deformation in corneal button and whole globes may be also due to the small, but significant $(\mathrm{p}=0.018)$ differences in radii of curvature between the corneal button fixed in the artificial chamber and in whole globes ( $R_{\text {Button }}=9.18 \pm 0.52$ versus $R_{\text {Globe }}=8.90 \pm 0.31 \mathrm{~mm}$ ). The difference observed in corneal thickness between whole globes and corneal button is surprising. Corneal thickness might have increased in buttons as the tissue when cut out is allowed to relax for a short time. The corneal deformation pattern in in vitro and in-vivo human 
eye globes did not show a significant difference in the maximal peak distance and in the temporal symmetry. The trend (non-significant) for a larger corneal deformation in vivo than in vitro could be associated to the age-differences between both groups. The most relevant difference was the larger deformation remaining after the direct air-puff response in vivo. This observation can be attributed to the contribution of the ocular muscles acting as an external viscoelastic damper. The addition of the corneal deformation to the damping of the ocular muscles may also explain small differences observed in the maximum corneal apex indentation. Differences are minor despite the great differences in handling and conditions of in vitro and in vivo human corneas. On the one hand, the in vitro eyes had been stored for up to 24 hours before measurement. This explains the increased corneal thickness due to hydration (8.1). On the other hand, in vitro eyes were significantly older than in vivo measured eyes, and slight differences in corneal stiffness with aging are expected.[59] Differences between deformation patterns of porcine and human corneas are consistent with prior knowledge of interspecies corneal biomechanical properties. Porcine corneas deformed to a larger extent and slower than human corneas. This confirms the previously reported difference in viscoelastic behavior between human and porcine cornea: porcine corneas relax more than human corneas (under a given stretch ratio).[231]

In comparison to the Ocular Response Analyzer (ORA), the corneal response following an air-puff was imaged with a higher temporal and spatial resolution in this study allowing the direct acquisition of corneal deformation patterns. To date, there is no clear evidence that the parameters obtained from the ORA (so-called corneal hysteresis $\mathrm{CH}$, and corneal resistance factor CRF) reveal constant and significant changes with CXL.[213, 230] Although alternative parameters can be extracted from the ORA signal,[199] the limitation of the system remains that only the temporal change of the slope of the corneal tangent in a small zone is measured. This limitation can be overcome by imaging the entire corneal 
Chapter 8. Contributing factors to corneal deformation in air-puff measurements

deformation within a zone of $8 \mathrm{~mm}$ of diameter, which gives access to higher number of descriptive parameters of the deformation. The high temporal and spatial resolution of the corneal deformation measurements upon air-puff ejection, such as those presented in the current study, and, in particular, the numerous experimental conditions tested, serve as excellent input data for inverse finite element models, which then will allow retrieving the corneal biomechanical properties (elastic and viscoelastic parameters, i.e. elasticity modules and Prony / relaxation time constants) and identifying the effect of side factors, such as the IOP, geometry and boundary conditions. Other emerging alternatives to measure corneal biomechanical properties in -vivo include Brillouin microscopy[180] and ultrasound,[215] which primarily address the measurement of the static elastic modulus. Corneal deformation measurements with air-puff give access to the dynamic behavior of the corneal tissue and therefore reveal different biomechanical properties than the before mentioned systems. Corneal dynamics, including viscoelasticity, have been studied primarily in -vitro[17, 74, 108, 231] and only been addressed in -vivo through the use of the ORA system.[199, 213, 230] In particular, it is expected that corneal changes following CXL treatment affect the viscoelastic properties to a large extent[17] (Richoz O., 2012, 8th CXL-Congress). Therefore the information that can be obtain from high resolution imaging of the corneal deformation with air-puff will make a valuable contribution in the characterization of the corneal biomechanicis in vivo.

In conclusion, the meaningfulness of deformation parameters obtained from the presented air-puff measurements depends on their correlation with the biomechanical properties of the corneal tissue. We identified the IOP to be mostly correlated with the maximal indentation depth of the apex, whereas corneal rigidity was best identified by the temporal symmetry factor. The maximal corneal peak distance was correlated to the maximal indentation depth of the apex. The contribution of the ocular muscles acted as an external damper and, when subtracted, the corneal deformation pattern is primarily driven by the real corneal 
response. Corneal viscoelastic properties are revealed by the temporal symmetry factor and changed after cross-linking. Our results allow a better clinical interpretation of the observed corneal deformation. This data can serve as input data for numerical and analytical models describing the cornea with the aim of predicting surgical outcomes more accurately. 
Chapter 8. Contributing factors to corneal deformation in air-puff measurements 


\section{Non-invasive in vivo evaluation of corneal viscoelastic biomechanical parameters}

In this study we present a numerical model in order to better understand the corneal deformation observed following an air-puff and analyze possibilities to retrieve biomechanical parameters out of the temporal and spatial deformation profile.

This chapter is based on the publication by S. Kling et al, entitled "Non-invasive in vivo evaluation of corneal viscoelastic biomechanical parameters", which is currently in preparation. Co-authors are N. Bekesi, C. Dorronsoro, D. Pascual and S. Marcos.

The author of this thesis (i) designed the study (ii) did the finite 
Chapter 9. Non-invasive in vivo evaluation of corneal viscoelastic biomechanical parameters

element simulation in collaboration with N. Bekesi and (iii) prepared the manuscript. 


\section{Introduction}

The demand for measuring biomechanical properties of biological tissue in vivo and non-invasively is high, because many physiologic and pathophysiologic processes alter the biomechanical properties of tissue. Abnormal tissue biomechanics play a key role in a wide range of diseases such as cystic fibrosis, osteoporoses, or aneurisms, among others. Also an alteration in the stress distribution to higher tension forces around tumors can favor their progression [24]. As tumor tissue is typically stiffer than normal tissue [13], measurement of tissue elasticity is used to diagnose cancer. Biomechancial measurements are also tracked to assess the function of muscles [129]. In dermatology, the characterization of skin biomechanics allows monitoring the effects of disease, wound healing [31], aging or cosmetics [18]. In ophthalmology, understanding of ocular biomechanics is essential in the research of underlying mechanisms, clinical evaluation, prognosis and treatment of multiple ocular conditions including myopia, glaucoma or corneal dystrophies. The cornea is a transparent optical and biomechanical structure. Pathological weakening of the corneal tissue in particular appears to be responsible for the corneal bulging, and therefore for the dramatic optical and visual degradation in keratoconus. Corneal collagen cross-linking is an emerging treatment for keratoconus attempting to increase corneal stiffness [194]. In addition, the mechanical response to surgical modification of the cornea, as scaffold for the major refractive surface of the eye, influences its optical performance. Surgical and disease models that integrate corneal geometrical data, sub-structural anatomy, elastic and viscoelastic properties have the potential to improve clinical outcomes of treatments such as corneal refractive surgery (i.e. by excimer ablation or femtosecond cuts) or intrastromal implants. However the predictability of these models will largely depend on the identification of pre-operative predictors of biomechanical response in individual patients. Today, most information regarding corneal biomechanical properties comes from ex 
Chapter 9. Non-invasive in vivo evaluation of corneal viscoelastic biomechanical parameters

vivo corneal strip extensiometry, or whole eye or corneal button inflation. These techniques have revealed important deficits in elastic tensile strength in keratoconus, and increased stiffness following cross-linking. However, these techniques are only applicable in vitro, where corneal properties may be affected by hydration state and other non-physiological conditions, which are not applicable in vivo. Several approaches have been suggested to measure corneal biomechanical properties in vivo, including corneal videokeratography upon stepwise central indentation with a cantilever [76] that measures corneal bending-resistance; ultrasonic [215] and magnetic resonance [123, 138] techniques that use the shear wave propagation velocity to map corneal elasticity in vivo; corneal optical coherence elastography producing a strain mapping based on speckle tracking; phase-sensitive Optical Coherence Tomography on phase changes of successive corneal cross-sectional images; and Brillouin microscopy [178], providing a spatially resolved map of corneal Brillouin modulus, which is correlated to the local relative values of corneal elastic modulus, upon induction of quasi-static oscillations $(\mathrm{GHz})$ in the tissue. A drawback of several of these techniques is that they are slow (magnetic imaging or Brillouin microscopy), show a relatively low spatial resolution (ultrasonic / magnetic resonance imaging) or require contact of a probe with the patient's corneal surface and hence the use of anesthetics (physical applanation and ultrasound-based techniques). Despite the wide variety of techniques, all of them only address the corneal elastic properties. However in the cornea and most soft-biological tissues viscoelastic properties, arising from the time-dependent nature of the biomechanical properties, are expected to be of major importance. For example, progressive deformation of the cornea (ectasia) occurring in keratoconus, and after some LASIK procedures, is a result of a viscoelastic phenomenon. Factors contributing to viscoelastic properties in the cornea may include the degree of collagen interweaving, keratocyte density, and the presence of hydrophilic proteoglycans, and their alteration may result in the viscoelastic failure or abnormal repair [50]. Certain treatments 
such as UV corneal collagen cross-linking likely modify both elastic and viscoelastic properties. A fast non-invasive technique to monitor corneal biomechanical properties is dynamic imaging of tissue deformation upon air-puff. Studying the deformation following an air-puff has recently been proposed in different biomedical areas [18, 65, 110]. For example, a focused air-puff system combined with a phase-sensitive optical coherence tomography system which captures the propagation velocity of surface waves, has been developed to quantify non-invasively the elasticity of soft tissue tumors [216]. Air-puff deformation is also used in other fields beyond medical diagnostics, such as to study chicken embryogenesis [205], or to monitor fruit firmness [71] or meat tenderness [122]. In most cases the degree of deformation of the sample is empirically related to mechanical parameter, but a retrieval of the inherent mechanical parameters of the tissue has been rarely attempted. To our knowledge, only Boyer et al [18] has proposed an analytical estimation of the "restricted Young's modulus" from experimental deformation curves in skin. Air puff applanation of the cornea is a frequent technique in ophthalmology to measure intraocular pressure. In these systems, intraocular pressure estimates are obtained from corneal deformation, assuming certain corneal mechanical properties and correction formulae to account for the influence of geometrical factors. Recently, corneal dynamic imaging upon deformation with an air puff has been presented. These systems use either high speed Optical Coherence Tomography [45] or a Scheimpflug imaging system [93] (a slit lamp camera with a tilted imaging plane) provided with a high speed camera as imaging devices to acquire dynamic images of the cross-sections of the cornea during the air-puff deformation event. Recent experimental studies confirm that the spatial and temporal deformation pattern of the cornea highly depends on factors beyond the inherent mechanical properties, such as corneal geometry, thickness and intraocular pressure. However, creating diagnostic tools for corneal disease or building predictive eye models for the biomechanical response to treatment require measuring the inherent 
Chapter 9. Non-invasive in vivo evaluation of corneal viscoelastic biomechanical parameters

mechanical properties of tissue. In this study we present a viscoelastic finite element model that predicts the geometrical spatial and temporal deformation of the cornea upon air-puff ejection. The model is validated with experimental deformation data of porcine eyes ex vivo, at different intraocular pressures, and of human eyes in vivo. A sensitivity analysis allows identifying the geometrical and biomechanical parameters that dominate the corneal deformation pattern. Being able to retrieve dynamic material properties will open a new way for tissue characterization in vivo, where interactions of molecular structure and extracellular matrix can be separated. Obtaining the relative modulus and relaxation time to describe the viscoelastic material properties will be key in the diagnosis of progressive corneal diseases, where these parameters could allow a prognosis of the severity and progression speed, and serve as a guidance for treatment.

\section{Eye Modelling}

(a)

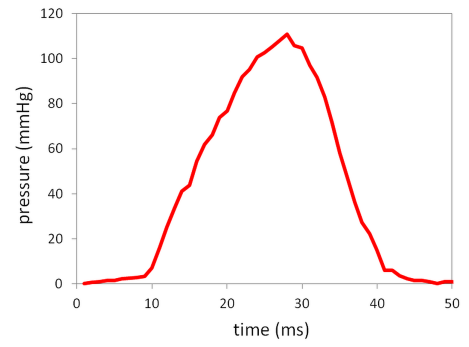

(b)

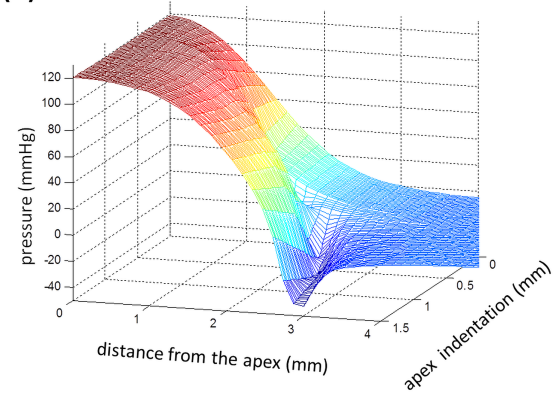

Figure 9.1: Air-puff characterization. (a) Experimentally measured temporal air-puff profile; (b) Results from CFD simulation showing the air-puff as a function of apex indentation and location along the cornea (horizontal distance from the apex).

Corneal deformation was studied in a finite element model with a two-dimensional axis-symmetric geometry. Initial corneal curvature and 
thickness dimensions were obtained from the experimental Scheimpflug cross-sectional images of the anterior segment of the eye in the horizontal meridian. Scleral dimensions were set to human and porcine eye dimensions reported in the literature. Table 9.1 summarizes the geometries for the different in vivo and in vitro conditions.

\section{Air-puff}

The air-puff was simulated a pressure applied on the anterior corneal surface, and varied as a function of time, distance to the apex and current corneal shape, see Figure 9.3. Figure 9.1 (a) depicts the experimentally measured temporal air-puff pressure profile at the center of the air-puff. A maximal air-puff pressure of $70.8 \mathrm{mmHg}$ at the corneal surface was taken. The geometry-dependent spatial pressure profile was obtained from a separate computational fluid dynamics (CFD) simulation, for a set of different deformed corneal geometries. Figure 9.1 (b) shows the resulting spatial air-puff profile expressed as a function of maximal corneal indentation at the apex and distance from the apex.

\section{Different corneal conditions}

In this section the finite element model was fitted to experimental corneal deformation data [110] obtained in a previous study, in which the temporal and spatial deformation profile of the cornea was measured with a high-speed Scheimpflug camera. Changes in the spatial and temporal deformation patterns were observed for different intraocular pressures (IOPs), boundary conditions and after cross-linking.

\section{Different intraocular pressures (IOPs)}

We simulated the corneal deformations upon the air-puff for different IOPs (ranging from 15 to $35 \mathrm{mmHg}$, i.e. covering the IOP range from physiological values to those found in severe glaucoma). We found that the differences between maximum corneal apex indentation was 
Chapter 9. Non-invasive in vivo evaluation of corneal viscoelastic biomechanical parameters

(a)

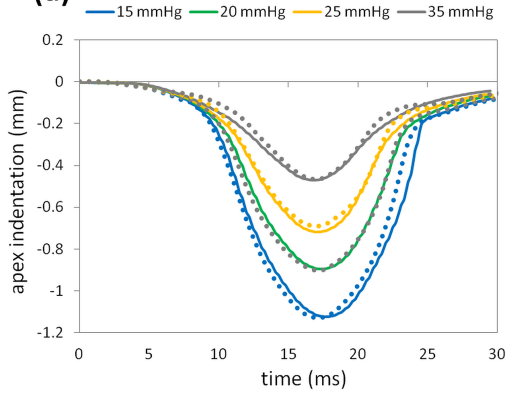

(b)

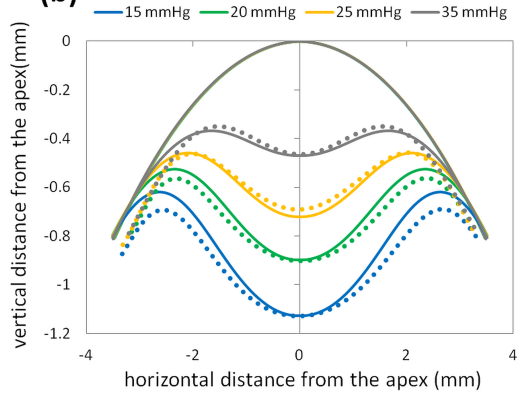

Figure 9.2: Corneal deformation in porcine eyes in vitro at different IOPs. Experimental data (dotted lines) and simulation results (continuous lines) for a cross-linked porcine cornea: (a) apex indentation as a function of time, (b) spatial profile at maximal deformation.

Table 9.1: Model parameters. The table lists the biomechanical and geometrical parameters used to simulate the human and pig corneal deformation.

\begin{tabular}{l|cc} 
& human & porcine \\
\hline condition & virgin & cxl \\
anterior elasticity modulus $(\mathrm{MPa})$ & 0.65 & 0.85 \\
difference between anterior and posterior & 1.16 & 30 \\
relaxation time $(\mathrm{ms})$ & 2 & 1.5 \\
relative modulus & 0.65 & 0.7 \\
material damping $(\mathrm{ms})$ & 0.01 & 1.3 \\
corneal thickness $(\mu \mathrm{m})$ & 558 & 211 \\
anterior curvature $(\mathrm{mm})$ & 8.03 & 8.06 \\
posterior curvature $(\mathrm{mm})$ & 6.86 & 7.54 \\
corneal diameter & 10 & 12 \\
scleral diameter & 19.5 & 23.5 \\
\hline
\end{tabular}


$1.13 \mathrm{~mm}$ for the lowest IOP and $0.46 \mathrm{~mm}$ for the highest IOP. Air-puff maximum pressure and, to a lesser extent differences in stiffness between the anterior and posterior cornea were found to play a major role in the predicted corneal deformation. Figure 9.2 shows the simulated corneal deformation $\left(E_{\text {anterior }}=25.5 \mathrm{MPa} ; E_{\text {posterior }}=0.85 \mathrm{MPa}\right.$ ) compared to experimental corneal deformation [110] in cross-linked porcine corneas in vitro for different IOPs. Both the decreased corneal apex indentation ( $\Delta$ fem $-0.676 \mathrm{~mm}$ versus $\Delta$ exp $-0.666 \mathrm{~mm}$ ) with increased IOP from 15 to $35 \mathrm{mmHg}$ and the decreased peak distance, i.e. horizontal distance between the corneal bending points at maximal deformation ( $\Delta$ fem -2.04 $\mathrm{mm}$ versus $\Delta \exp -2.34 \mathrm{~mm}$ ) are well reproduced by the model.

The relation between corneal indentation and eye globe compression (resulting from scleral deformation) depended on the IOP, but also on the difference between the anterior and posterior corneal stiffness. At $15 \mathrm{mmHg}$ the corneal deformation accounted for $48.9 \%$, while at 35 $\mathrm{mmHg}$ only for $7.80 \%$. Also, the higher the difference between the anterior and posterior corneal stiffness, the less dominant was the corneal indentation (i.e. for $E_{\text {anterior }} / E_{\text {posterior }}=1$ : corneal deformation $=52.0$ $\%$; for $E_{\text {anterior }} / E_{\text {posterior }}=1.16$ : corneal deformation $\left.=48.9 \%\right)$. The viscoelastic effect was evidenced by the remaining deformation following the air-puff event (which accounted for between 18.7 and $24.1 \%$ - for different IOPs- of the remaining indentation, and gradually decreased with time). This viscoelasticity described by a relative modulus of 0.7 and a relaxation time constant of $1.5 \mathrm{~ms}$, contributed $46 \%$ to the maximal apex indentation.

\section{Different boundary conditions}

Changes in the boundary conditions were implemented by simulating the human cornea (I) under natural fixation, i.e. by the ocular muscles and (II) within the eye, i.e. surrounded by the sclera in order to model the experiments with in vivo and enucleated eyes, respectively. Corneal in vivo deformation is compared to simulations with damping of the entire 
Chapter 9. Non-invasive in vivo evaluation of corneal viscoelastic biomechanical parameters

ocular globe, while corneal in vitro deformation is compared to simulations without damping (see Figure 9.4). We found that ocular muscles contributed with $0.112 \mathrm{~mm}$ to the apex displacement and induced a hysteresis (additional to the viscoelastic hysteresis of the cornea) in the zone of indirect air-puff response.

\section{What determines the corneal response?}

Table 9.2: Parameter gradients. The table shows the result of the sensitivity analysis.

\begin{tabular}{|c|c|c|c|c|c|}
\hline$\%$ & $\begin{array}{c}\Delta \text { peak } \\
\text { distance } \\
(\mathrm{mm})\end{array}$ & $\begin{array}{c}\Delta \text { apex } \\
\text { Adentation } \\
(\mathrm{mm})\end{array}$ & $\begin{array}{c}\text { physio- } \\
\text { logic } \\
\text { range }\end{array}$ & $\begin{array}{c}\Delta \text { peak } \\
\text { distance } \\
(\mathrm{mm})\end{array}$ & $\begin{array}{c}\Delta \text { apex } \\
\text { indentation } \\
(\mathrm{mm})\end{array}$ \\
\hline thickn & -7.1685 & 2.0108 & $\Delta 100 \mu m$ & -0.7168 & 0.2011 \\
\hline stiffness (MPa) & -3.6603 & 1.9088 & $\triangle 0.2 \mathrm{MPa}$ & -0.7218 & 0.3764 \\
\hline $\begin{array}{l}\text { relative modulus } \\
\text { (no unit) }\end{array}$ & 0.8929 & 1.9088 & $\Delta 1$ & 0.8929 & 0.1000 \\
\hline $\begin{array}{l}\text { relaxation } \\
\text { time (ms) }\end{array}$ & 0.0430 & -0.0232 & $\Delta 10 m s$ & 0.4300 & -0.2324 \\
\hline IOP $(\mathrm{mmHg})$ & -0.0618 & 0.0232 & $\Delta 10 \mathrm{mmHg}$ & -0.6180 & 0.2320 \\
\hline curvature $(\mathrm{mm})$ & 0.1875 & -0.0163 & $\Delta 1 \mathrm{~mm}$ & 0.1875 & -0.0163 \\
\hline density $10^{6} \mathrm{~g} / \mathrm{m}^{3}$ & 0.1577 & -0.0030 & $\Delta 500 \mathrm{~kg} / \mathrm{m}^{3}$ & 0.0008 & 0.0000 \\
\hline
\end{tabular}

Corneal biomechanical response varied across different treatments and conditions. A sensitivity analysis was performed for the human eye in vivo (i.e. with damping) in order to evaluate which geometrical and biomechanical parameters determine the corneal deformation with an air-puff. Figure 9.5 presents the predicted dependency of the apex indentation and peak distance (distance between the highest lateral points of the cornea at maximal deformation) on different geometrical and biomechanical parameters. Table 9.2 shows the slopes of the linear regressions to the data shown in Figure 9.5. Dependencies on absolute parameter variations were investigated. The left side in the graph 
represents data for parameter variations by $40 \%$, and the right side for parameter variations within the estimated physiological range. The sclera also had a significant effect, increasing the apex indentation by $0.20 \mathrm{~mm}$ when changing its rigidity by $90 \%$.

\section{Discussion and conclusions}

New imaging acquisition techniques such as a high speed Optical Coherence Tomography or high speed Scheimpflug imaging allow capturing the dynamic geometrical deformation response of the cornea. To our knowledge, we have presented for the first time numerical estimates of corneal elastic and viscoelastic parameters, based on Scheimpflug corneal deformation imaging (spatial and temporal) and sophisticated Finite Element Modeling. The numerical viscoelastic model reproduces with high accuracy both the temporal variation of corneal indentation and its spatial profile upon air-puff for different corneal deformation patterns. The estimated biomechanical parameters describe the inherent viscoelastic tissue properties, which are independent on the corneal geometry. Cross-linked porcine corneas were 1.31 times more rigid than virgin corneas confirming previous reports. Discrepancies, especially in the spatial deformation, were observed for very hydrated corneas, suggesting that swelling (in contrast to dehydration such as after crosslinking) induces changes in the material model that are not captured in the current simulation. In previous studies, static [6] and time dependent $[17,59]$ material properties of the corneal tissue have been addressed. However the dynamic response of a material can be very different from its static behavior; viscoelastic materials typically behave more rigid the faster a loading condition is applied. Dynamic analysis hence will give information on the instant rigidity of a material, while static material properties represent the elasticity at infinity. As dynamic analysis is performed at high speed, there is no time for osmotic pressure changes - related to the diffusion of water within the tissue - to 
Chapter 9. Non-invasive in vivo evaluation of corneal viscoelastic biomechanical parameters

produce any effect. Hence the dynamic properties of tissue are likely dominated by the extracellular matrix, while static properties give more information on the collagen structure. Combining static and dynamic analysis therefore might allow a better understanding of the interaction between the extracellular matrix with the collagen structure. The air-puff corneal imaging technique addresses dynamic properties of tissue in a similar time range $(20 \mathrm{~ms})$ as ultrasound-based elastography $(50 \mathrm{MHz})$ and magnetic resonance imaging $(300 \mathrm{MHz})$ and of a wider time range than Brillouin microscopy $(\mathrm{GHz})$. However, the new technique surpasses the first two in patient comfort, and the second two in acquisition rates. Besides, all these previous techniques attempted to estimate only the linear modulus of elasticity and neglected tissue viscosity. Our approach allowed retrieving corneal viscoelasticity (which we found to be mainly driven by the hysteresis remaining after the air-puff) and corneal elasticity (mainly determined by the amount of apex indentation). Although the sensitivity analysis showed a strong correlation between the corneal rigidity and its deformation, geometrical parameters, such as the central corneal thickness, as well as IOP, also had a large effect. This means that all factors need to be considered and entered as input parameters in the simulation, which restricts the complexity of the finite element simulation. We believe that in vivo dynamic analysis is an extremely valuable clinical diagnostic tool for corneal pathology, patient screening for refractive surgery, as well as guidance and evaluation of treatment. In particular, the air-puff technique is a promising approach to measure the viscoelastic properties on surfaces, unavailable from shear wave propagation analysis or Brillouin microscopy. Besides, the use of an air-puff makes the technique non-invasive and particularly well-suited to easily accessible tissues such as the cornea. Compared to previous analytical approximations, finite element modeling reduces largely the amount of approximations needed. Improvements in the current strategy for the currently highly computationally demanding optimization procedure, will allow bringing the technique to a stage where corneal biomechanical 
parameters are readily measured in the clinic.

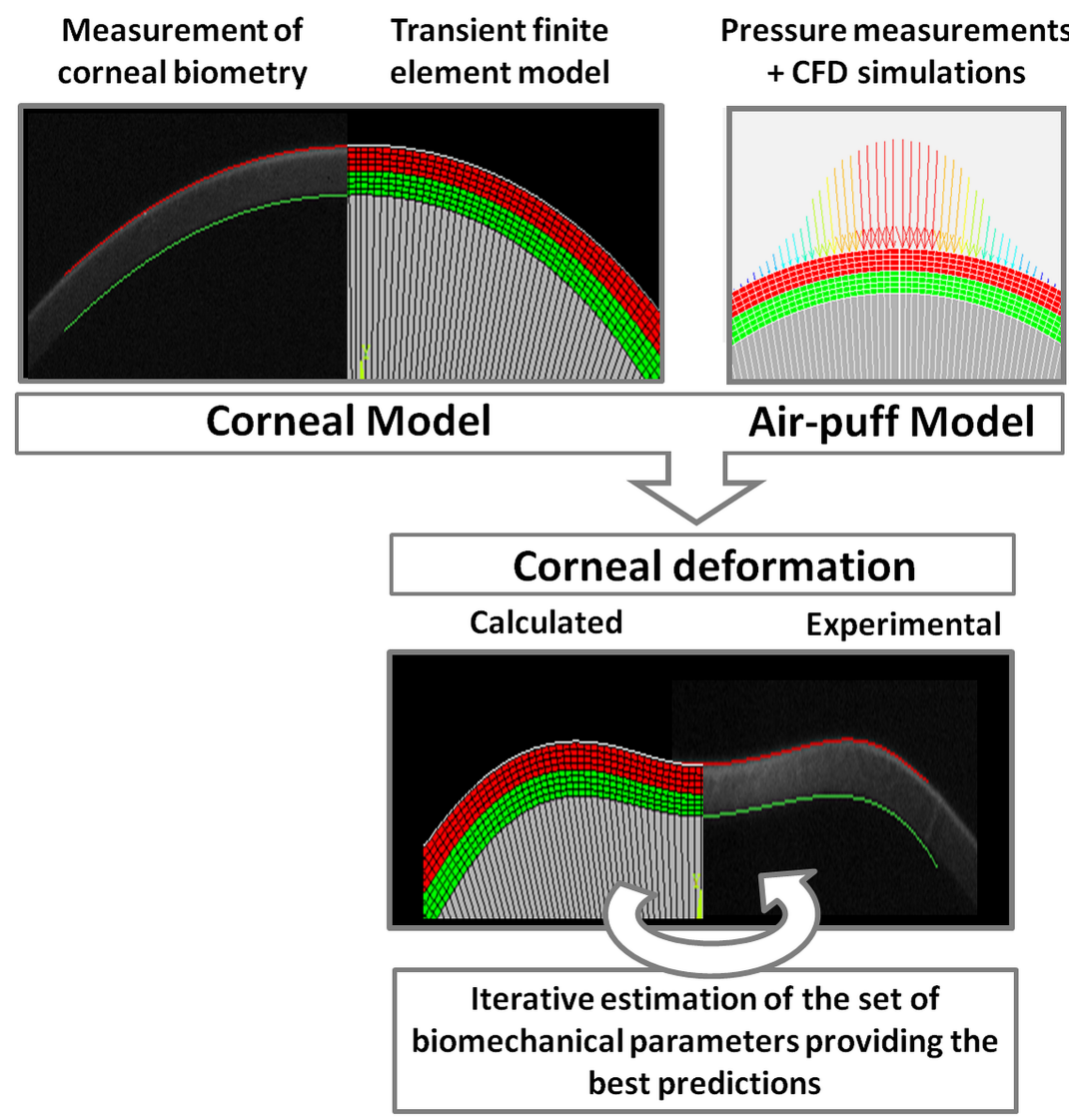

Figure 9.3: Schematic of the simulation procedure. (1) corneal geometric data from Scheimpflug images are used to define the model geometry. Inverse modeling is performed to account for the effect of applying the IOP; (2) the temporal pressure profile measured experimentally and the spatial pressure profile obtained from CFD simulation are applied to the cornea as a function of time, location and current deformed shape; (3) the finite element model is solved for the current parameter set; (4) the simulation results are compared to the experimentally measured deformation. A step-wise optimization approach is used to find the parameter set that leads to the most similar deformation. 
Chapter 9. Non-invasive in vivo evaluation of corneal viscoelastic biomechanical parameters

(a)

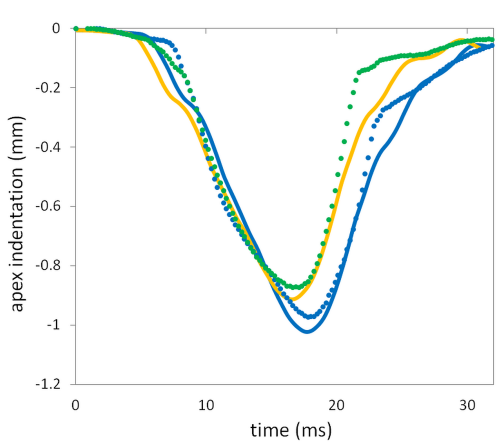

(b) — - in-vivo — in-vivo (without damping) $\ldots$ in-vitro

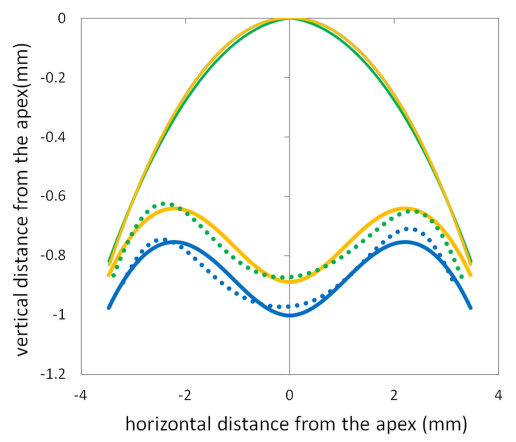

Figure 9.4: Corneal deformation pattern in human eyes in vivo. Experimental data (dotted lines) and simulation results (continuous lines) for the human cornea: simulations show the condition with and without ocular muscle damping; experimental data show in-vivo deformations (measured in patients) and in-vitro deformations (measured as an enucleated whole globe).
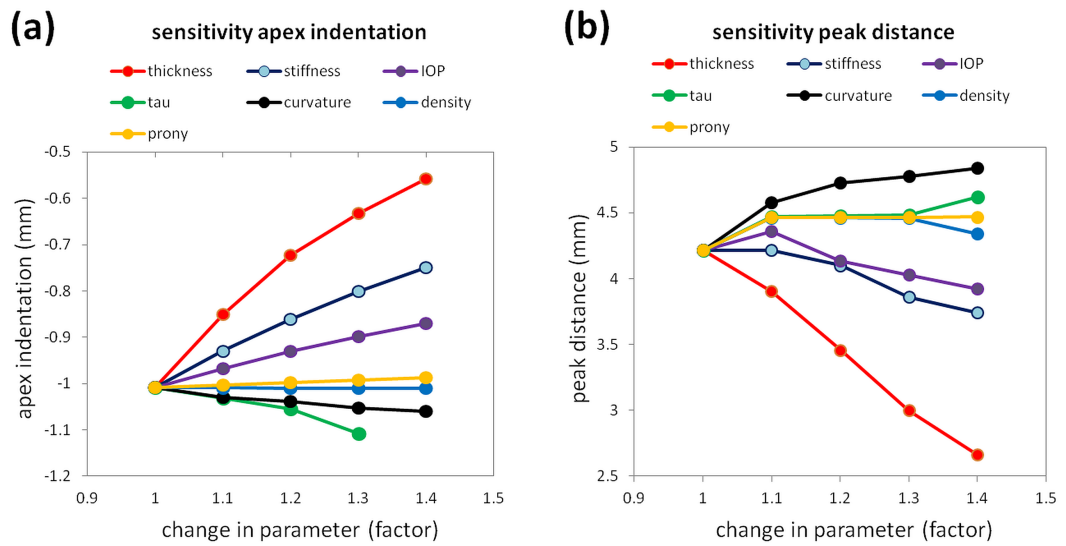

Figure 9.5: Sensitivity analysis for geometrical and biomechanical parameters. (a) for the maximal apex indentation, (b) for the maximal peak distance. 


\section{Methods}

\section{Air Puff Imaging}

The Corvis system (Oculus, Wetzlar, Germany) uses high-speed Scheimpflug imaging to capture the spatial and temporal corneal deformation following an air-puff. Typically 140 images are taken during the $\sim 25 \mathrm{~ms}$ deformation event (i.e. at a speed of about 5600 images/sec).

\section{Subjects and experimental data}

Experimental corneal deformation data following an air-puff were taken from our previous study [104]. In this study in vitro porcine corneas were measured under different hydration (after photosensitizer $0.125 \%$ riboflavin-20\%-dextran instillation), stiffness (after UV collagen crosslinking), boundary conditions (corneal button, in vitro eye globe, in vivo eye-globe) and intraocular pressures (IOP) in order to evaluate the effect of the different parameters on the corneal deformation pattern. For this study we used a sub-set of these experimental data: (1) in vitro cross-linked pig eyes, and (2) in vivo human eyes. Corneal experimental input parameters include the undeformed corneal geometry, the temporal profile of corneal apex indentation and the spatial corneal deformation pattern.

\section{Air-puff characterization}

A pressure sensor (MPX2301DT1, Freescale Seminconductor Inc, Tempe, AZ, USA) was used to measure the central temporal pressure distribution of the air-puff at $11 \mathrm{~mm}$ distance (typical position of the cornea) from the air-tube. The temporal pressure profile was roughly fit by a linear function, with a slope of $5.38 \mathrm{mmHg} / \mathrm{ms}\left(R^{2}=0.9768, \mathrm{p}<0.001\right)$ for pressure increase and $-8.38 \mathrm{mmHg} / \mathrm{ms}\left(R^{2}=0.9796, \mathrm{p}=0.03\right)$ for pressure decrease, respectively. The measured duration of the air-puff was $20.63 \mathrm{~ms}$ (97\%) and $27.5 \mathrm{~ms}$ (100\%). In order to determine the 
Chapter 9. Non-invasive in vivo evaluation of corneal viscoelastic biomechanical parameters

geometry-dependent spatial shape of the air-puff at different stages of the deformed cornea, a computational fluid dynamics (CFD) simulation of the interaction between the air puff and a set of deformed corneal geometries was performed. The maximum air speed $(115 \mathrm{~m} / \mathrm{s})$ was estimated by Bernoulli's equation based on the measured pressure of the air puff. This value is comparable with the air speed measured previously [133] using a hot wire anemometer next to the tube end (> $100 \mathrm{~m} / \mathrm{s}$ ). In order to determine the pressure distribution of the air puff we performed a set of CFD simulations. We found that the shape of the pressure distribution does not change significantly with varying the flow speed, but it alters for different deformed shapes of the cornea. In the finite element simulations, the applied pressure was a function of the apex indentation and location (see Figure $9.1 \mathrm{~b}$ ). This pressure function was then normalized and multiplied by a scaling factor Pmax.

\section{Finite element simulation}

\section{Geometry}

A 2D axis-symmetric eye model was defined corresponding to the different corneal conditions and boundaries (see Table 9.1 for parameter details of the eye model). For whole eye globes, the outer coat of a half eye globe was considered, consisting of cornea, limbus and sclera. Corneal curvatures were adjusted to match the experimental values after IOP application. Scleral geometry was taken from the literature [144] and limbus was defined by connecting cornea and sclera.

\section{Material models}

The corneal tissue was described by a linear viscoelastic material; limbus and sclera were described by a purely elastic material. It should be noted that these material models represent the macroscopic response of the biological tissues. The microscopic structure was not considered within the short time scales analyzed in this study. The cornea was divided in 
two equal layers (anterior and posterior, extending $50 \%$ of the entire corneal depth each) and a different elastic modulus was assigned to each. The aqueous and the vitreous body were modeled by a hydrostatic fluid with a pressure equal to the IOP. Biomechanical parameters of the sclera and limbus (see Table 9.1) were defined by data obtained from the literature [46]. The corneal density was set to $\rho_{\text {normal }}=1062 \frac{\mathrm{kg}}{\mathrm{m}^{3}}$ [103] for a $700 \mu \mathrm{m}$ corneal thickness and scaled according to the thickness values in the experiments.

\section{Boundary conditions and loads}

For the in vitro whole globe condition a part of the posterior sclera was fixed similar to the fixation in the eye-holder. For the in vivo condition the eye globe was damped along on the vertical symmetry axis. The intraocular pressure was applyied to the interior surfaces of cornea, limbus and sclera.

\section{Air-puff application}

A pressure load was applied on the element edges of the anterior surface of the cornea according to the spatial pressure distribution of the airpuff at the different indentation depths. This was necessary because the fluid dynamics characteristics change significantly as the cornea deforms. Furthermore the pressure variation with time (measured experimentally with the pressure sensor) was considered by multiplying the current pressure with the normalized temporal pressure profile.

\section{Ocular muscle contribution}

The ocular muscles were simulated by a mass-less longitudinal springdamper, defined by a spring constant $(5.106 \mathrm{~N} / \mathrm{m})$ and a damping coefficient (1.0). 
Chapter 9. Non-invasive in vivo evaluation of corneal viscoelastic biomechanical parameters

\section{Load steps}

In the first load step the IOP was applied to the model. Then, in the next step the load modeling the air-puff was applied. In order to achieve sufficient temporal resolution, this step was divided into 49 sub-steps, so the applied pressure was updated every $625 \mu \mathrm{s}$.

\section{Parameter selection}

A step-wise optimization approach was applied in order to fit the numerical response to the experimental behaviour: First, the initial radii of curvature (anterior, posterior) were adjusted so that after applying the intraocular pressure, both the modelled and experimental corneal geometry (within the 8-mm central zone) was identical. Second, the elastic modulus was used to adjust the absolute amount of indentation. Third, Pmax was adjusted in order to reporduce the desired difference in maximal indentation depth when changing the IOP from 15 to 35 $\mathrm{mmHg}$. (This step was performed only once and then the parameters of the air-puff were kept constant for all conditions), Fourth, the viscoelastic parameter set was adjusted in order to get the hysteresis in the temporal deformation profile, which is observed after the air-puff stops, i.e. in the zone of indirect air-puff response. Fifth, a difference in stiffness between the anterior and posterior corneal areas was introduced in order to adjust the spatial corneal deformation profile. In normally hydrated corneas the anterior cornea is approximately $16 \%$ more rigid. In cross-linked corneas the difference between the anterior and posterior corneal stiffness is larger, as typically only the $60 \%$ of the entire cornea is stiffened after treatment [184].

\section{Analysis}

The deformed geometry obtained from the FE-simulation was exported and analyzed in terms of the temporal deformation profile and the spatial shape at maximum deformation. After selecting the model parameter set 
that best represented the virgin human in vivo condition, a sensitivity analysis was performed in order to determine the geometrical and mechanical parameters that dominated the shape and amount of corneal deformation following the air-puff. Seven parameters were selected (corneal thickness, stiffness, curvature, density, IOP and two viscoelastic constants), changed consecutively by up to $+40 \%$ and the effect on the overall deformation was determined.

\section{Computing techniques}

ANSYS APDL structural mechanics code (ANSYS, Inc., Canonsburg, PA) was used for the mechanical simulations. The CFD simulations were carried out using the FLUENT module of the ANSYS software package. The analysis of the deformed corneal shape was performed in Matlab (The MathWorks, Natick, MA). 
Chapter 9. Non-invasive in vivo evaluation of corneal viscoelastic biomechanical parameters 


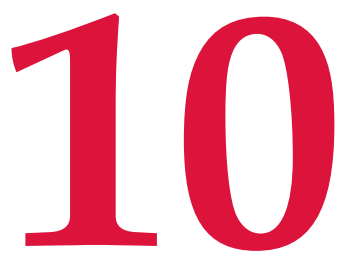

\section{Non-invasive quantification of}

\section{corneal biomechanical properties}

using OCT-Vibrography

In this study we present a novel technique to measure the dynamic biomechanical parameters of the corneal tissue. It is a more direct approach compared to the analysis fo the corneal deformation following an air-puff and hence is less affected by side factors.

This chapter is based on the publication by S. Kling et al, entitled "Non-invasive quantification of corneal biomechanical properties using OCT-Vibrography" submitted to Journal of the Royal Society Interface. Co-authors are I.B. Akca, E.W. Chang, G. Scarcelli, N. Bekesi, S.H. Yun and S. Marcos. This work was also presented in a poster contribution at 
Chapter 10. Non-invasive quantification of corneal biomechanical properties using OCT-Vibrography

the 2013 Annual Meeting of the Association for Research in Vision and Ophthalmology (ARVO) in Seattle (Washington, USA).

The author of this thesis (i) designed the study, (ii) did the finite element simulation, (ii) did the sample preparation, (iii) performed the experiments in collaboration with I.B. Avci, E. Chang and G. Scarcelli, (iv) did the data analysis in collaboration with I.B. Avci and E. Chang and (v) prepared the manuscript. 


\section{Introduction}

Corneal biomechanics are essential for the characterization of the cornea prior to and following treatments, such as after refractive surgeries (LASIK, PRK, ICRS implantation), UV collagen cross-linking treatment, keratoplasty, and in certain pathologies that compromise corneal structure (such as keratoconus). Measuring corneal biomechanical properties allows a better prediction of surgical outcomes, helps improving the understanding of collagen cross-linking and could permit the early diagnosis of keratoconus. A current limitation in measuring corneal biomechanics is that very few systems are applicable in-vivo. Two of these commercially available systems (Ocular Response Analyzer [125], CORVIS [110]) analyze the geometrical corneal deformation following an air-puff. This measure however is dependent on several side factors, such as IOP, the presence of the sclera and the dynamics of the ocular humors. Ultrasound techniques [215] estimate corneal stiffness from analysis of the shear wave, but they are limited by a relatively low imaging resolution and the need of direct contact to the eye, which reduces patient's comfort. Another promising technique addressing the in-vivo measurement of quasi-static (cornea undergoes $\mathrm{GHz}$ vibration) corneal biomechanical properties is Brillouin microscopy [177]. Both, the dynamic and static properties of the cornea are of interest, especially as several in-vitro studies suggest a change in the corneal viscoelasticity after cross-linking. Viscoelasticity describes a time dependent material property, which can be assessed only in a dynamic measurement. Ideally, the viscoelastic properties could be used to predict the temporal evolution of the corneal shape under a given intraocular pressure, and be indicative for a pathologic cornea. In mechanical engineering, modal analysis [61] is typically applied to study the dynamic behavior of a material [28]. Thereby the natural frequencies are identified by exciting the sample mechanically at different frequencies and by recording the oscillation amplitude at given locations. The measured frequency response function depends on the 
Chapter 10. Non-invasive quantification of corneal biomechanical properties using OCT-Vibrography

inherent mechanical properties of the material. In a recent study a novel imaging approach has been presented to measure the function of the eardrum by imaging the modal shapes occurring during vibration [30]. A phase-triggered optical coherence tomography (OCT) technique termed OCT vibrography was used to capture the motion while the eardrum was exposed to sound emitted from a loudspeaker. It has also been shown that this technique can induce vibrations in the eye globe and that different harmonics can be observed in the range from 50 to $1050 \mathrm{~Hz}$. In the current study we predict the corneal response to vibration using numerical simulations. In addition, we evaluated the ability of the method to isolate corneal biomechanical properties from other parameters. Pilot experimental data are presented to verify the numerical results.

\section{Protocol}

A numerical model was built in order to describe the vibration behavior of the cornea. Experiments were performed using a custom-developed high speed Optical Coherence Tomography (OCT) system synchronized with a loudspeaker. The measured frequency response functions were taken as input to fit the biomechanical parameters of the model, so to match the experimental and simulated vibration response. The retrieved set of parameters was used in a sensitivity analysis to study the factors that determine the natural frequencies. The effect of corneal elasticity on the vibration response was studied in in anterior and posterior corneal flaps, and virgin and cross-linked corneas, where differences in the biomechanical response are expected between corneal regions and with cross-linking treatment.

\section{Finite element (FE) simulation}

Resonance occurs if a system is excited by one of its natural frequencies, which depend on the interaction of the material's stiffness and mass. Modal analysis was performed - using proprietary software (ANSYS 
APDL 14.0 - Academic; ANSYS, Inc., Canonsburg, PA)) - to describe this context. A more detailed description of the theoretical formulation can be found in section 1 . This analysis allows studying the vibration characteristics, i.e. natural frequencies and mode shapes of a structure. The imposed frequencies were selected in the range of the experimentally scanned frequencies $(50-700 \mathrm{~Hz})$. As damping increases the width of the resonance peaks, the frequency spacing was tested between 1 and 50 $\mathrm{Hz}$ to find the minimally necessary but least computationally demanding frequency step size

\section{Geometry and boundaries}

Two different axis-symmetric 2D finite element models were built to simulate (I) corneal flaps and (II) whole eye globes. The flap geometries consisted of a rectangular cross-section of the circular corneal flap. The fixation in the flap holder was implemented by constraining the displacement at the external border to zero. The eye globe geometry consisted of a quarter of the anterior eye coat, including cornea, limbus, sclera, and aqueous humor. Table 10.1 shows the main numerical values of the model. It was assumed that the anterior and posterior cornea has different stiffness, as suggested by Hennighausen et al [86]. Geometries of the cornea, limbus and sclera were set to values taken from the literature [144]. Thereby the deformations occurring due to prestressing the tissue with the IOP were considered. The ocular geometry in the relaxed eye was defined according to Table 10.1. The interior of the eye coat was defined as a fluid, simulating the aqueous and vitreous humors. An IOP of $15 \mathrm{mmHg}$ was applied. In order to prevent global motion, the sclera was constrained at a circular ring of $1 \mathrm{~mm}$ width at a distance of 5 $\mathrm{mm}$ from the apex, representing the fixation by the eye holder.

\section{Material model and biomechanical properties}

Due to the nature of modal analysis, material nonlinearities were not considered. Although the cornea is prone to have non-linear properties, 
Chapter 10. Non-invasive quantification of corneal biomechanical properties using OCT-Vibrography

the small experimental deformations observed during sound excitation, i.e. $\pm 10 \mu \mathrm{m} \mathrm{m}$ vertical displacement, which indicates much lower values of longitudinal strain, suggest that it is possible to assume a linear elastic material model in these simulations. Corneal stiffness was left as a variable in order to adjust the modal response to the experimentally measured frequency response function. Stiffness for the limbus and sclera was fixed and set to Elimbus $=37.2 \mathrm{kPa}$ and Esclera $=79.2 \mathrm{kPa}$, respectively. A Poisson's ratio of $v=0.499$ and a density of $\rho=1160 \mathrm{~kg} / \mathrm{m}^{3}$ were used for all ocular tissues. The temporal non-linearity of the corneal tissue was implemented by using a viscoelastic material model, which was described by a two-parameter Prony series ( $1=0.1 / \tau 1=1 \mathrm{~ms}$ ). The relaxation time $(\tau)$ was chosen in the range of the sound frequencies, and the relative modulus (p) was set within the range of the experimentally observed peak width values. An acoustic fluid material model was applied to describe the aqueous and vitreous humors. Fluid material properties as shown in Table 10.1.

\section{Analysis}

The modal analysis allowed retrieving the natural frequencies and modal shapes for corneal flaps and whole eye globes under different conditions. A sensitivity analysis was performed to study the dependency on different geometrical (thickness, curvature) and biomechanical (corneal stiffness, density) parameters, as well as other side factors (IOP, presence of sclera).

\section{Experiments}

In the measurements the corneal stifness used for the simulation was adjusted in order to confirm the predictions of the natural frequencies and to evaluate differences in the frequency response function. 


\section{Phase-sensitive OCT}

A phase-sensitive OCT was used for the measurements. It is an updated version of the system presented by Chang et al in 2012 [30]. Briefly, the scanning beam of a swept source OCT was triggered to the sound wave emitted from a loudspeaker in order to image a single period of the oscillation with phase reference at consecutive locations along the cornea (see also chapter 2).

\section{Experimental protocol}

Measurements were performed for a frequency scan range of the loudspeaker from 240 to $520 \mathrm{~Hz} / 655 \mathrm{~Hz}$ with $\Delta \mathrm{f}=40 \mathrm{~Hz}$ (in flaps) and from 50 to $510 \mathrm{~Hz}$ with $\delta \mathrm{f}=20 \mathrm{~Hz}$ (in eye globes). The frequency ranges were selected according to estimations where the natural frequencies might be found. In whole eyes, for each frequency a cross-section of the central cornea (diameter $6-8 \mathrm{~mm}$ ) was imaged at a sound volume of 100 to $110 \mathrm{db}$. As a reference - in order to discount potential motions of the entire set-up - a rigid glass mounted next to the cornea was included at the border of each A-scan. In flaps, the central zone with a diameter of 3 $-4 \mathrm{~mm}$ was imaged. Here the sound volume was lower, which made the glass reference unnecessary in this condition.

\section{Eyes / conditions}

A total of 5 freshly enucleated eyes ( 2 procine, 3 bovine) were used within 1 to 12 hours post-mortem. Different boundary conditions for the cornea were tested: (i) 2D corneal flap mounted in a custom holder; (ii) eye globe mounted in a customized holder. Flaps (about $120 \mu \mathrm{m}$ ) were obtained with a custom-built microkeratome (Deriva, Valencia, Spain) from the anterior and posterior cornea. Eye globes were prepared and measured in three consecutive steps: (I) virgin state without any modification; (II) after de-epithelialization and instillation of $0.125 \%$ Riboflavin10\%Dextran for 30 minutes; (III) after irradiation with UV-light (370 nm, 
Chapter 10. Non-invasive quantification of corneal biomechanical properties using OCT-Vibrography

$3 \mathrm{~mW} / \mathrm{cm} 2$ ) for 30 minutes. Performing step II and III is equivalent to the standard Dresden protocol for Collagen Crosslinking, which increases corneal stiffness, with the only difference that the Dextran content of the photosensitizer solution has been reduced from $20 \%$ to $10 \%$ in order to prevent excessive corneal dehydration. This set of different boundary and treatment conditions allowed studying the effect of geometry, corneal rigidity and changes due to dehydration.

\section{Data analysis}

The vertical (transversal) oscillation of the central cornea was analyzed for each frequency and condition: First, the oscillation of the glass reference was subtracted from the overall oscillation measured at the cornea. Then the resulting signal was Fourier transformed in order to extract the exact amplitude at the frequency of interest. This step was necessary as the loudspeaker did not emit a totally single-frequency signal, but contained contributions of higher harmonics at certain frequencies. In order to obtain the frequency response curve, the amplitude of the central corneal oscillation was plotted as a function of frequency.

\section{Results}

\section{Biomechanical properties}

Generally the elasticity modulus was related to a shift in the natural frequencies, while the viscoelastic parameters determined the width of the individual resonance peaks.

\section{Corneal flaps}

The best prediction of the experimental flap vibration response was obtained for corneal stiffness of Eflap $=14.58 \mathrm{kPa}$ in the simulation. This corneal stiffness was used as reference for a sensitivity analysis to study the relative contribution of different factors on the natural 
(a)

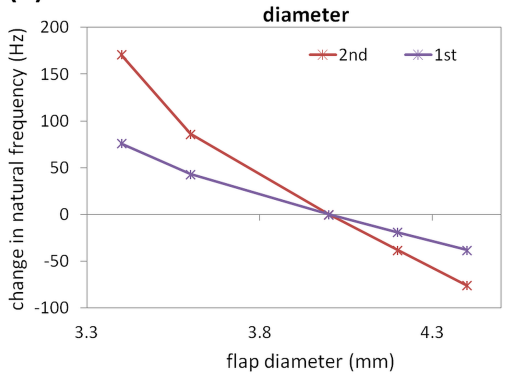

(c)

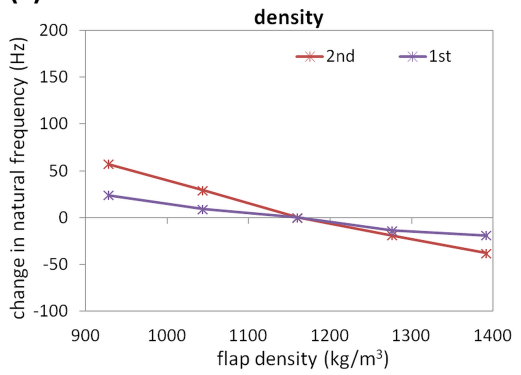

(b)

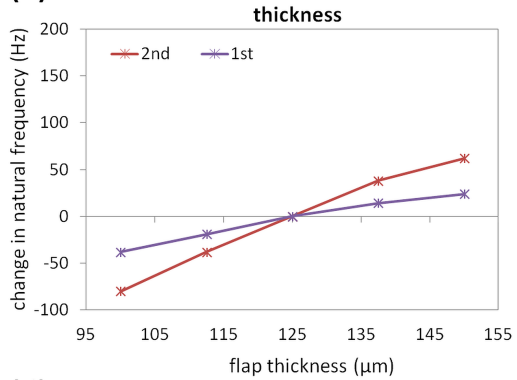

(d)

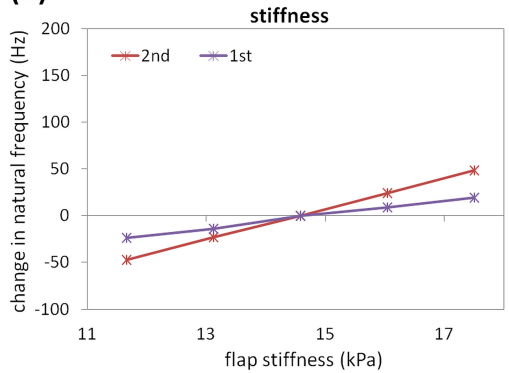

Figure 10.1: Predicted dependence of the change in 1st and 2nd natural frequencies with (a) flap diameter, (b) flap thickness, (c) flap density, and (d) flap stiffness, from a sensitivity analysis. 
Chapter 10. Non-invasive quantification of corneal biomechanical properties using OCT-Vibrography
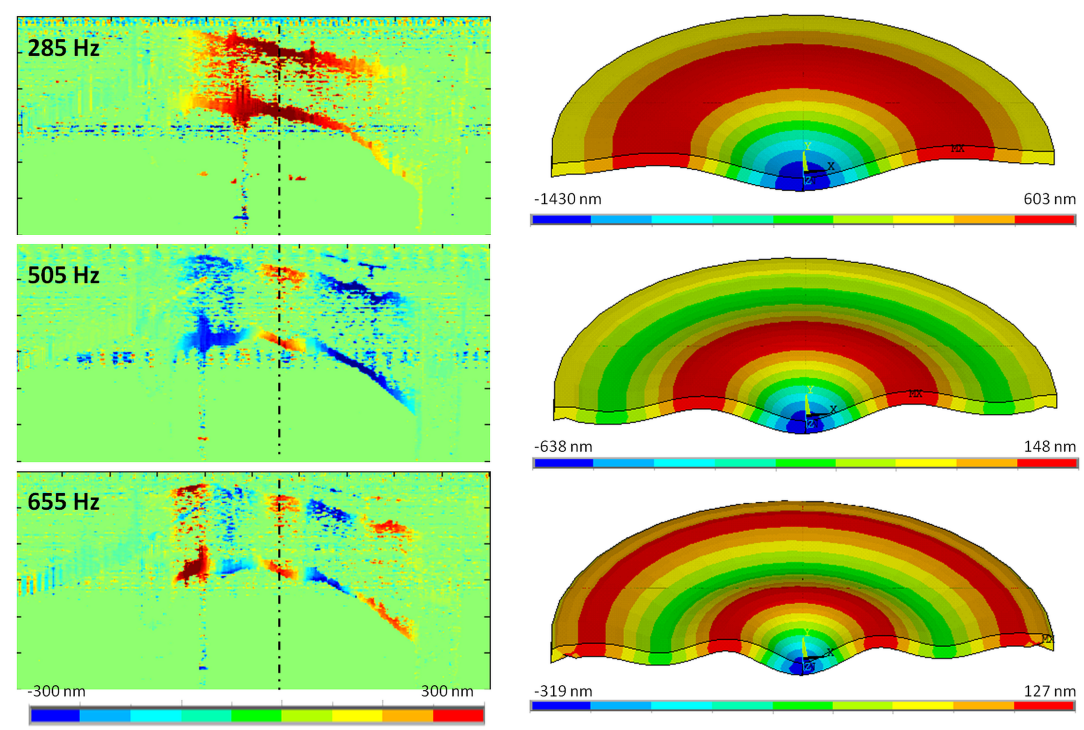

Figure 10.2: Left panel: Experimentally measured mode shapes at different frequencies in a posterior bovine flap. Right panel: Corresponding harmonics of the fundamental mode in corneal flaps, predicted from simulations in the range from 220 to $750 \mathrm{~Hz}$. 
frequencies and their shift. Figure 10.1 shows the observed dependencies for a parameter variation of $\pm 20 \%$ and Table 10.2 summarizes the slopes. The resonance frequency was most sensitive to the geometry of the membrane (its radius, followed by its thickness) and to a lesser exent, but still highly significantly, to density and corneal stiffness. Thereby the second resonance peak was more sensitive to changes in corneal stiffness than the first peak.

(a)

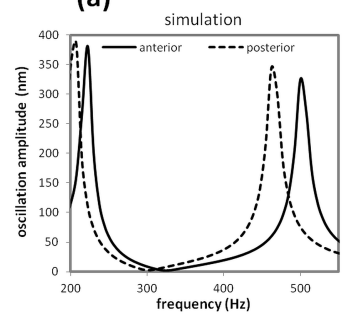

(b)

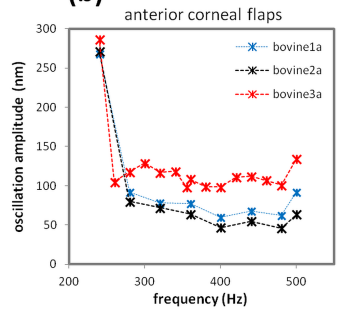

(c)

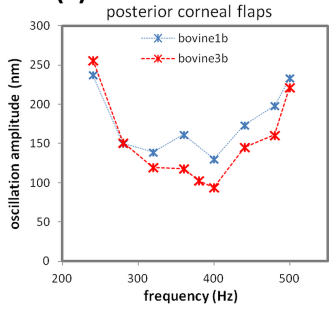

Figure 10.3: Frequency response function of the (a) simulated anterior and posterior corneal flaps, experimentally measured (b) anterior flaps, and (c) posterior bovine flaps.

Three different modes (harmonics of the fundamental mode) shapes were found within the range from 50 to $750 \mathrm{~Hz}$ in the numerical simulation (see Figure 10.2). Left panels illustrate the different mode shapes observed experimentally in a posterior flap. Figure 10.3(a) the predicted frequency response functions for corneal flaps. Simulations predicted well the experimental frequency response of anterior bovine flaps as shown in Figure 10.3(b). Posterior flaps (Figure 10.3(c)) showed a second resonance peak in the tested range, while anterior corneal flaps only showed one resonance peak. Optimal correspondence between the simulated and the experimental posterior cornea was obtained when the flap stiffness was decreased by a factor of 0.8 with respect to the anterior's flap (from $14.58 \mathrm{kPa}$ to $11.6 \mathrm{kPa}$ ). Each peak in Figure 10.3(a) corresponds to a different mode shape. This indicates that the first two modes in Figure 10.2 are presented near resonance, while the third mode is the beginning of its formation. 
Chapter 10. Non-invasive quantification of corneal biomechanical properties using OCT-Vibrography
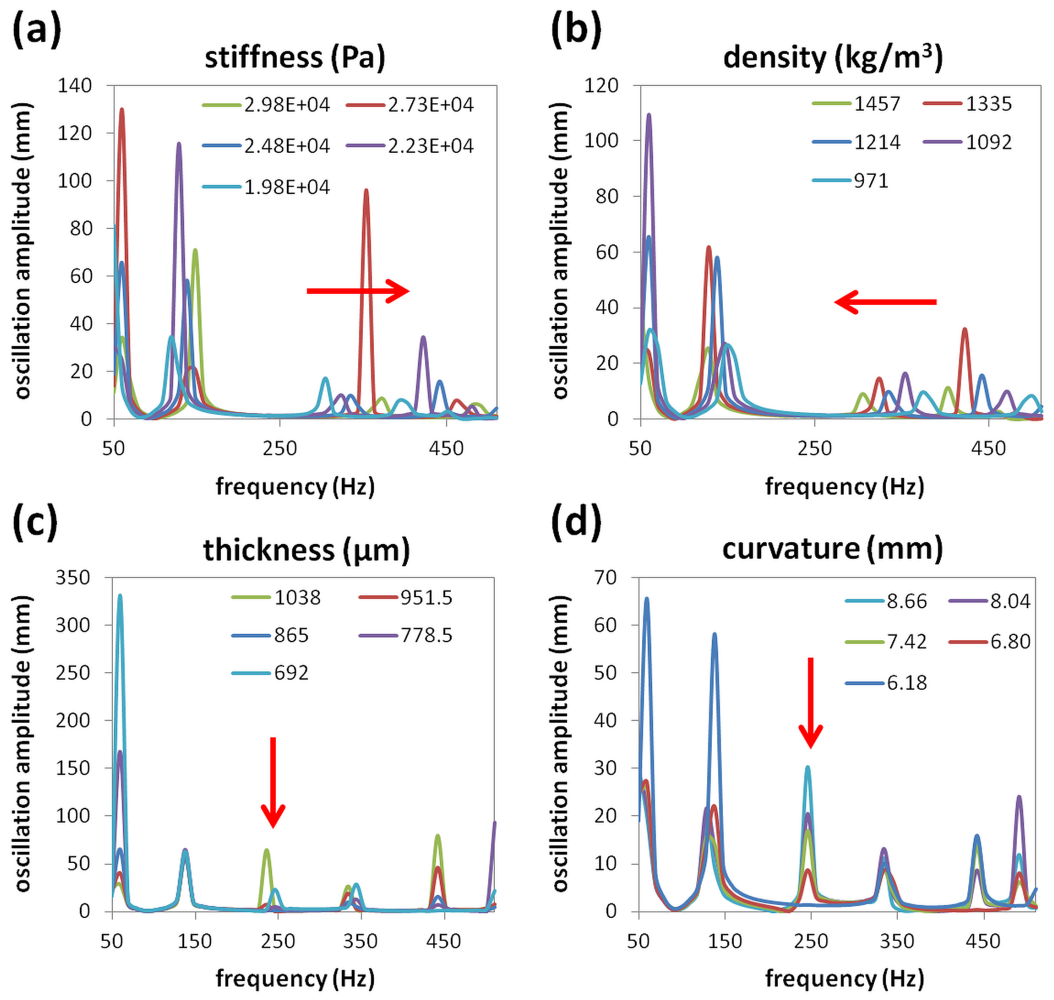

(e)

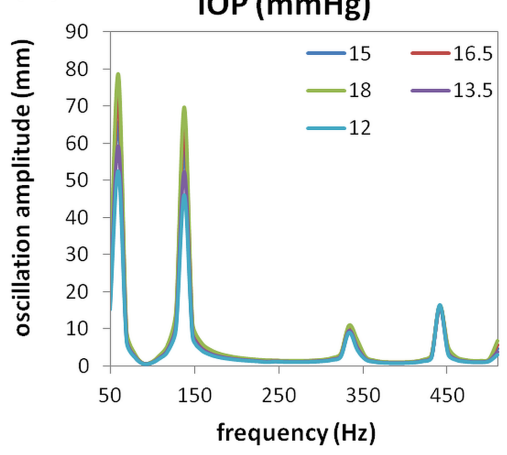

Figure 10.4: Simulated frequency response functions of whole eye globes for different (a) corneal stiffness, (b) density, (c) thickness, (d) curvature, and (c) IOP values. Red arrows indicate changes in the frequency response function when varying the corresponding parameter. 
Using a sampling rate of $40 \mathrm{~Hz}$ (as in the experiments) induced an estimated uncertainty of $4.37 \mathrm{kPa}(30 \%)$ in the retrieved corneal stiffness. As a general tendency, an increase in flap stiffness produced a shift of the resonance peaks and increased separation between peaks.

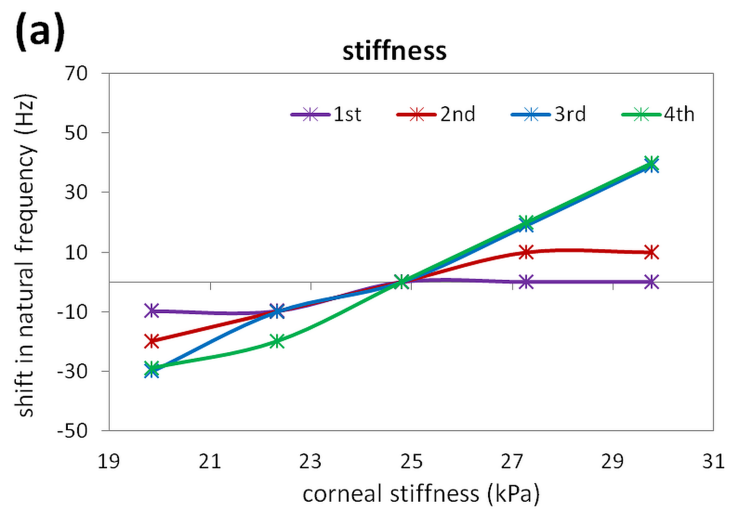

(b)

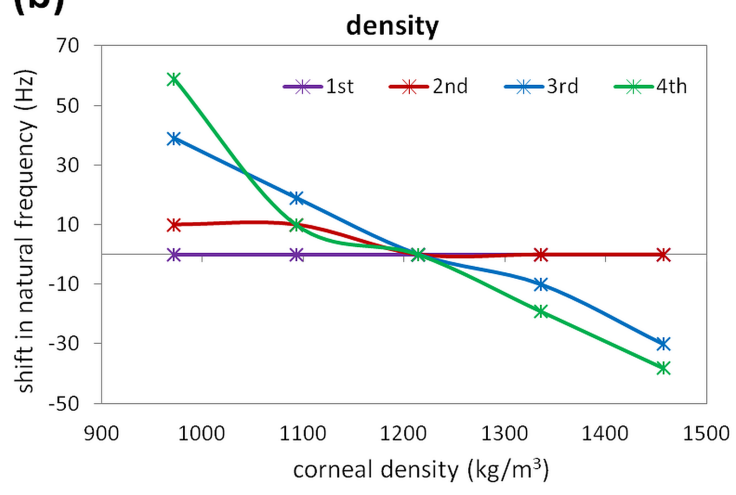

Figure 10.5: Predicted dependency of the natural frequency (1st-4th) shifts on (a) corneal stiffness and (b) corneal density.

\section{Whole eye globes}

The experimental oscillation response of eye globes was best reporoduced in the simulation with a corneal stiffness of $24.8 \mathrm{kPa}$ and $19.8 \mathrm{kPa}$ 
Chapter 10. Non-invasive quantification of corneal biomechanical properties using OCT-Vibrography
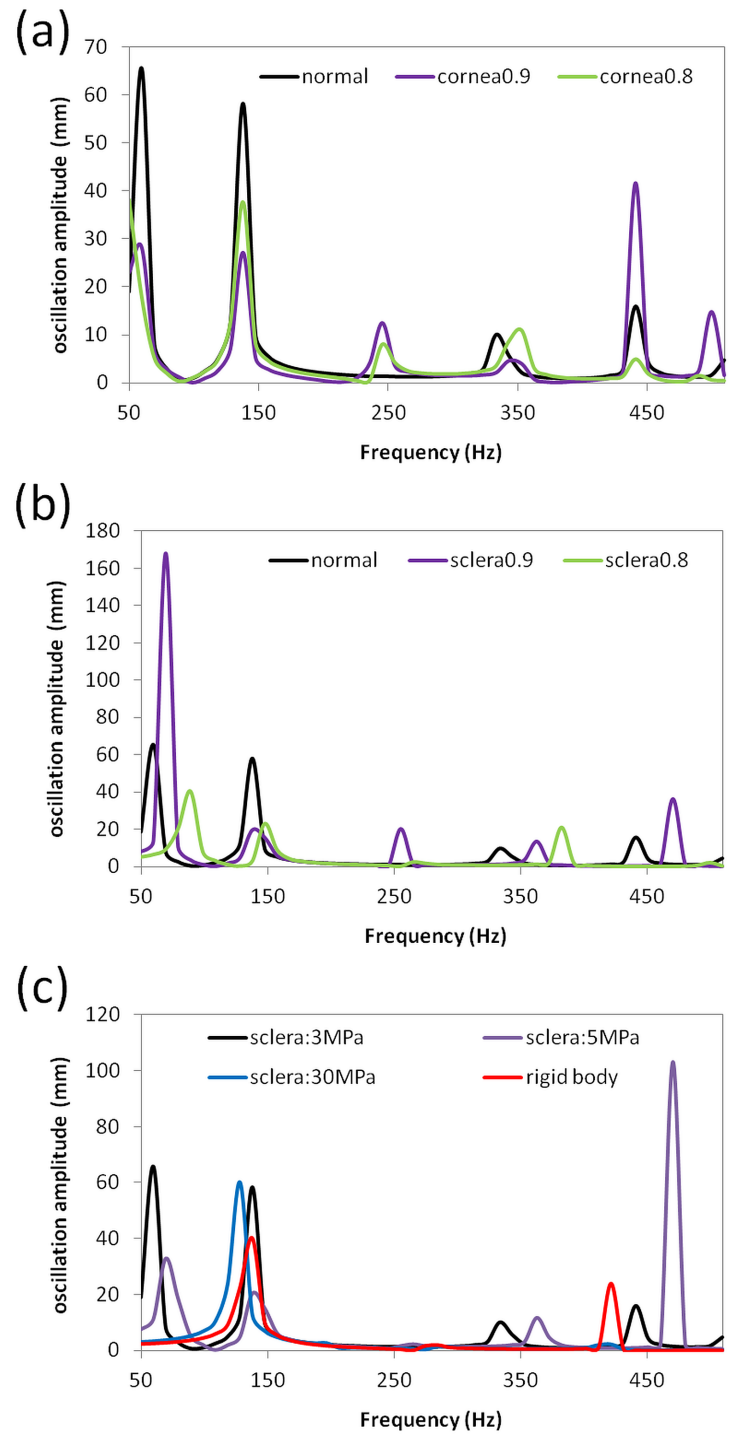

Figure 10.6: Predicted shifts in the frequency response function of the whole globe upon variations in (a) diameter of the cornea; (b) diameter of the sclera; (c) biomechanical properties of the sclera. The legend in (a) and (b) indicates the factor by which the dimensions of the cornea and sclera are modified. The legend in (c) indicate the scleral stiffness. 
for the anterior and posterior virgin cornea respectively. This corneal stiffness was used as reference for the sensitivity analysis. Figure 10.4 shows the resulting dependencies of the natural frequencies of a whole eye globe under different parameter variations (by $\pm 20 \%$ ). Thereby changes in corneal stiffness and density induced a shift in the natural frequencies showing a strong, linear dependency (see Figure 10.5). Table 10.2 summarizes the corresponding slopes of the linear trend. Corneal thickness and curvature played a role in the presence of the natural frequency at $250 \mathrm{~Hz}$, while the IOP had no significant effect.

Figure 10.6(a,b) show the simulated frequency response function of a whole globe when corneal and sclera dimensions were changed independently, which allowed us to evaluate the role of the geometry of each eye component. We observe that changes in corneal diameter induced predominantly changes in the resonance amplitude, while changes in the sclera dimension induced a shift of the natural frequency. The the role of the biomechanical properties of the scleraon the frequency response function of whole eye globes was further explored by simulating different scleral stiffness, i.e. low (74.4 kPa), normal (744 kPa), and an infinite (rigid body). Figure 10.6 (c) shows that scleral biomechanical properties played a role in the presence of the resonance peak at $59 \mathrm{~Hz}$ and induced a frequency shift, particularly at higher resonance frequencies. The different simulated mode shapes of whole eye globes at the observed within the frequency range from 50 to $510 \mathrm{~Hz}$ are shown in Figure 10.7.

Figure 10.8 compares the simulated frequency response functions for virgin and cross-linked corneas. An increase in corneal stiffness by a factor of 1.64 (from $24.8 \mathrm{kPa}$ to $40.6 \mathrm{kPa}$ ) predicted well the experimentally observed changes in the frequency response function after cross-linking. The experimental amplitudes are generally lower than in the simulations. The simulated modal analysis present the maximal possible oscillations, but in practice the amount of deformation depends on the volume of the sound wave, the energy coupling from sound to tissue, and the general damping of the sound wave within the material. Therefore the actual 
Chapter 10. Non-invasive quantification of corneal biomechanical properties using OCT-Vibrography
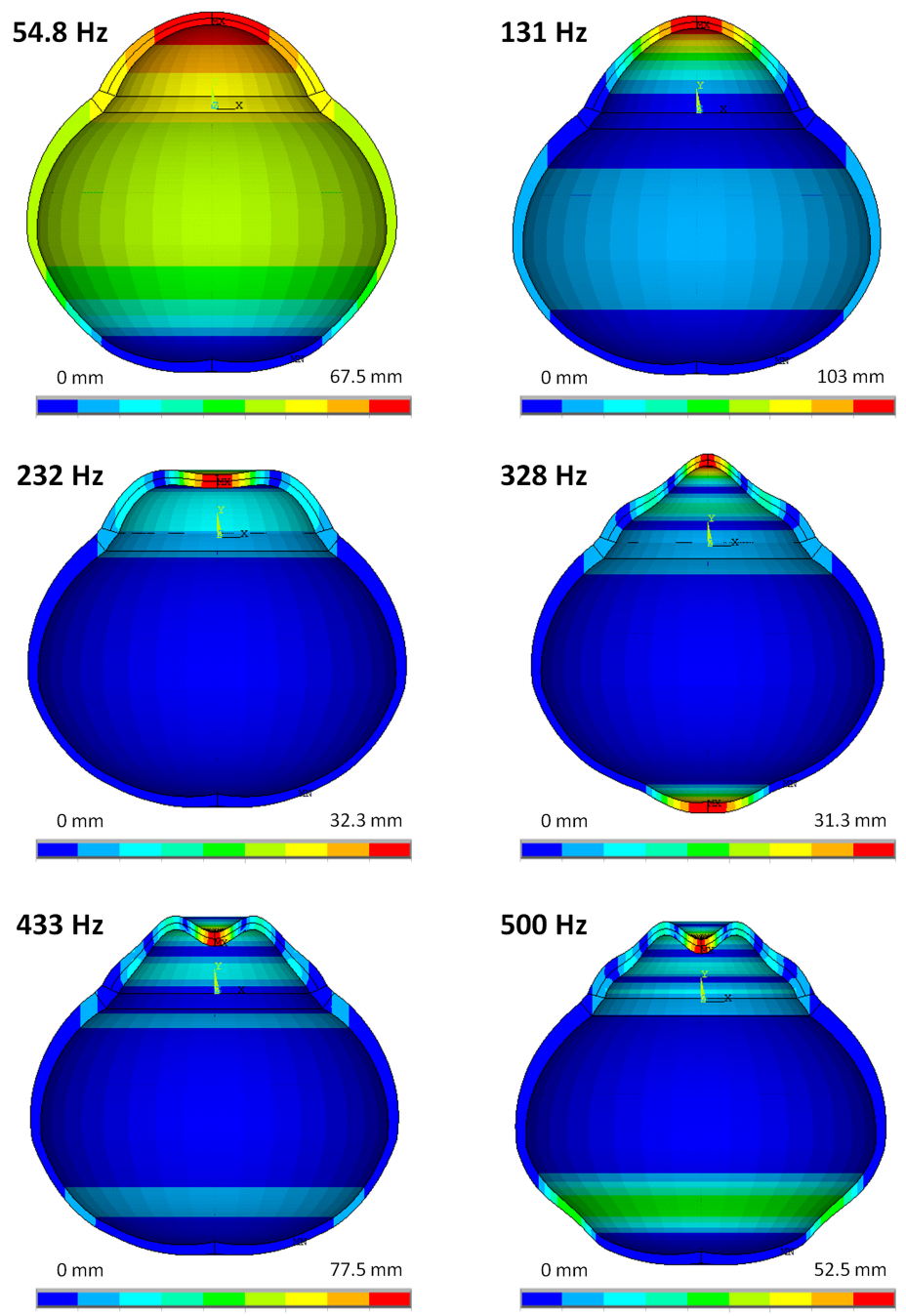

Figure 10.7: Harmonics of the fundamental mode in whole globes, predicted from simulation in the range from 50 to 510 $\mathrm{Hz}$. 

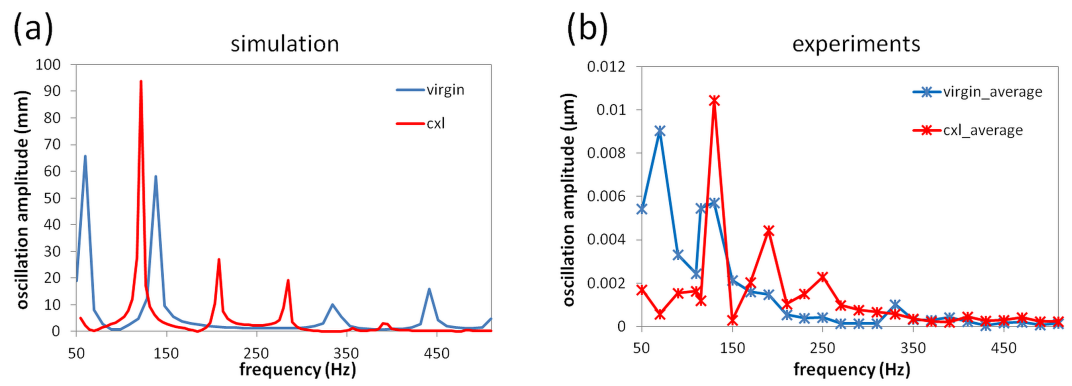

Figure 10.8: response function of the (a) simulated and (b) experimental (average of 2 eyes per condition) whole globes, virgin (in blue) and cross-linked (in red).

values of the oscillation amplitude are not important in this analysis, but the natural frequencies and the mode shapes are important.

Figure 10.9 shows changes in the frequency response function for a simulated simple keratoconic eye. Thereby panel (a) presents a central keratoconus, i.e. by reducing corneal stiffness in an angular zone from 70 to $90^{\circ}$, and panel (b) presents an idealized keratoconic cornea where the corneal stiffness has been reduced in a peripheral ring from 50 to $70^{\circ}$. Corneal stiffness was reduced in the selected area from 24.8 to 2.48 $\mathrm{kPa}$ for the simulattion. In both cases changes in the frequency response function were predicted: In the central keratoconus new resonance peaks appeared at 206 and $275 \mathrm{~Hz}$, and shifts occured for the resonances at 334 and $441 \mathrm{~Hz}$, while in the peripheral keratoconus a new resonance peak appeared at $187 \mathrm{~Hz}$ and shifts occured for the resonances at 138, 334 and $441 \mathrm{~Hz}$. In contrast to Figures 10.1 and 10.4, where the mechanical parameters were changed homogenously along the cornea, local variations in corneal stiffness in the simulations of keratoconic corneas resulted in different trends for the individual resonance modes, both in the direction of frequency shift and in the difference in amplitude across frequencies. Although the phase lag between stress (periodic application of force) and strain (subsequent deformation) is associated with the material's viscoelastic behavior, the experimentally observed 
Chapter 10. Non-invasive quantification of corneal biomechanical properties using OCT-Vibrography
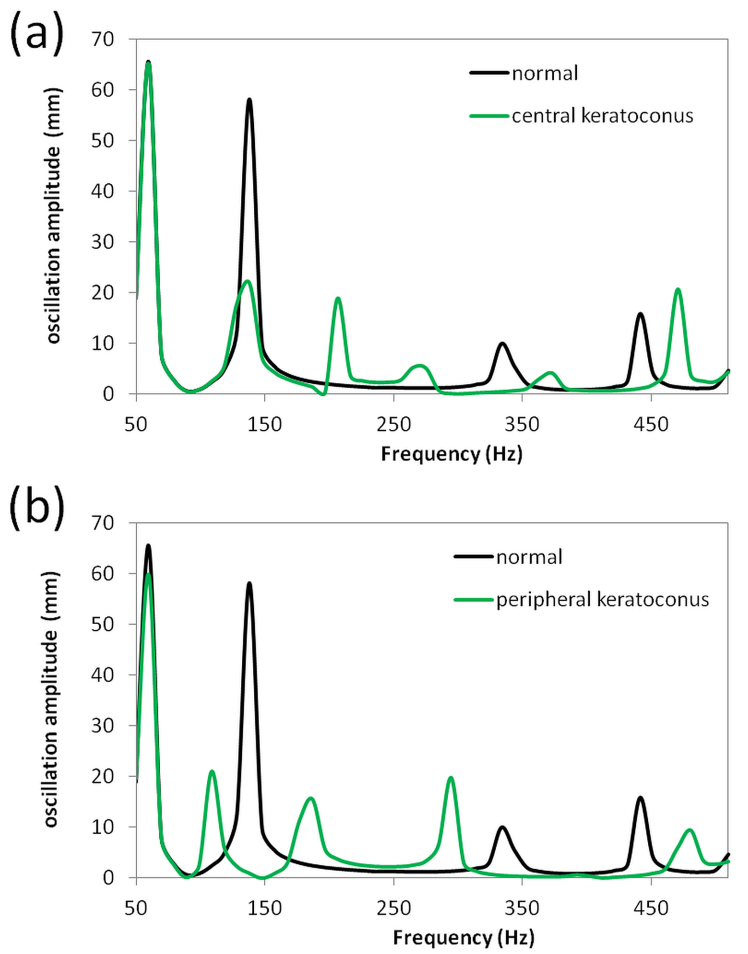

Figure 10.9: Predicted frequency response function in whole eye globes, for a normal cornea (black line) and for corneas with a localized reduction of corneal stiffness (green line), with the weakened area located (a) centrally (70$\left.90^{\circ}\right)$ or (b) in a peripheral ring $\left(50-70^{\circ}\right)$. 
phase lag did not show sufficient consistency to provide information regarding viscoelastic parameters, i.e. the ratio between the storage and loss modulus.

\section{Discussion}

Table 10.1: Sensitivity gradient of geometrical and biomechanical parameters respect to different resonance peaks, analyzed in a range of $\pm 20 \%$.

\begin{tabular}{|l|ccc|}
\hline & (unit) & flap & globe \\
\hline central thickness & $\mu \mathrm{m}$ & 120 & 865 \\
density & $\mathrm{kg} / \mathrm{m}^{3}$ & 1160 & 1160 \\
poisson's ratio & - & 0.499 & 0.499 \\
stiffness ant/post & - & 1 & 1.2 (virgin) / 2.4 (cxl) \\
relative modulus & - & 0.1 & 0.1 \\
relaxation time & $\mathrm{ms}$ & 1 & 1 \\
anterior curvature & $\mathrm{mm}$ & - & 6.8 \\
posterior curvature & $\mathrm{mm}$ & - & 5.57 \\
corneal diameter & $\mathrm{mm}$ & 4.0 & 10.4 \\
sclera diameter & $\mathrm{mm}$ & - & 19 \\
limbus stiffness & $\mathrm{kPa}$ & - & 37.2 \\
sclera stiffness & $\mathrm{kPa}$ & - & 79.2 \\
\hline & $(\mathrm{unit})$ & - & humors \\
\hline density & $\mathrm{kg} / \mathrm{m}^{3}$ & - & 1000 \\
sonic velocity & $\mathrm{m} / \mathrm{s}$ & - & 1480 \\
density & $\mathrm{kg} /(\mathrm{s} \cdot \mathrm{m})$ & - & $8.94 \mathrm{e}-4$ \\
\hline
\end{tabular}

We present a numerical model to study the natural frequencies of the cornea under vibration and propose a new technique to measure corneal biomechanical properties by non-contact. The advantage of this resonance-based approach is that the natural frequencies are directly related to the mechanical properties of the corneal tissue, and hence 
Chapter 10. Non-invasive quantification of corneal biomechanical properties using OCT-Vibrography

corneal OCT vibrography can be more directly associated to in-vivo corneal biomechanics than approaches relying on measuring the corneal deformation following an air-puff $[45,110,199]$ or the water volume needed to provoke a certain increase in the IOP[151]. We showed that natural frequencies are practically independent from other side factors such as the IOP, corneal thickness and curvature. The interactions between corneal deformation, thickness, and biomechanical properties in other techniques, such as tonometry, make the application specific correction formulae necessary [203]. However the current technique potentially allows the estimation of corneal biomechanical properties without the influence of corneal geometry or IOP. Corneal thickness is critical in the assessment of the real IOP. We showed a strong and consistent relation between the oscillation amplitude of the cornea and the actual IOP 10.4 .

Table 10.2: Sensitivity gradient of geometrical and biomechanical parameters respect to different resonance peaks, analyzed in a range of $\pm 20 \%$.

\begin{tabular}{lc|cccc|l}
\multicolumn{2}{c}{ flap } & 1st & 2nd & & unit \\
\hline stiffness & $14.58 \mathrm{kPa}$ & 7.7476 & 16.255 & & $\mathrm{~Hz} / \mathrm{Pa}$ \\
density & $1160 \mathrm{~kg} / \mathrm{m}^{3}$ & -93.95 & -205.1 & & & $\mathrm{~Hz} / \mathrm{mg} / \mathrm{km}^{3}$ \\
thickness & $125 \mu \mathrm{m}$ & 1256 & 2880 & & & $\mathrm{~Hz} / \mathrm{mm}$ \\
radius & $4.0 \mathrm{~mm}$ & -111.7 & -237.7 & & & $\mathrm{~Hz} / \mathrm{mm}$ \\
\hline \multicolumn{2}{c|}{ whole globe } & $1 \mathrm{st}$ & 2nd & 3rd & 4th & unit \\
\hline density & $1160 \mathrm{~kg} / \mathrm{m}^{3}$ & 0.00 & -24.71 & -137.56 & -183.69 & $\mathrm{~Hz} / \mathrm{mg} / \mathrm{km}^{3}$ \\
stiffness & $24.8 \mathrm{kPa}$ & 1.19 & 3.23 & 6.73 & 7.18 & $\mathrm{~Hz} / \mathrm{Pa}$
\end{tabular}

Therefore corneal OCT vibrography can be envisioned as a twoin-one diagnostic tool to assess (1) corneal stiffness by looking at the resonance frequency and (2) IOP by looking at the oscillation, however the contribution of thickness, curvature and corneal stiffness must be taken into account. The spatial shapes of the resonance modes in Figure 10.7 indicate that the deformation occurs primarily at the cornea, 
suggesting that the resonance modes are more sensitive to corneal than to scleral biomechanical properties. This was further confirmed by the sensitivity analysis, where a $40 \%$ change in sclera stiffness accounts for a frequency shift of $25 \mathrm{~Hz}$, while a the same amount of change in corneal stiffness accounts for a frequency shift of $49 \mathrm{~Hz}$. In flap simulations we observed the trend that the viscoelastic parameters were the related to the width of the resonance peaks and to the oscillation amplitude. Nevertheless, this effect is very much dependent on the used step width and was not further investigated. Also, the experimental step size was too large to retrieve the real width of the experimental resonance peaks. As shown in Figure 10.4, the frequency response function is also sensitive to local differences across the cornea. Weakening in a certain location will favor or hinder the formation of one mode or another. Although our 2D model only allowed to study axis-symmetric corneal weakening, the results suggest that OCT vibrography might also be useful in the diagnosis of keratoconus, as the oscillation pattern is affected by the location of the cone and the amount of local corneal stiffness decrease. Corneal stiffness estimated from the simulations of the experimental data differed between flaps and whole eyes. Possible reasons for the discrepancy include the fact that the flap is pre-stressed upon mounting in the holder, and that the corneal mechanical properties in the entire corneal vary along its thickness. Besides, the predictions indicate that a finer experimental frequency sampling is desirable to guarantee that all resonance peaks are captured within the scanned area. The oscillation amplitudes observed experimentally reach up to $300 \mathrm{~nm}$ (flaps) / $10 \mu \mathrm{m}$ (whole eye). These are larger than the oscillations induced by ultrasound, but orders of magnitude smaller than the corneal indentation induced by an air-puff tonometer, and hence very non-invasive to the corneal tissue. In corneal vibrography the oscillation amplitude depends on the actual stress-strain interactions within the corneal tissue, allowing a more direct estimation of the corneal mechanical properties. Ultrasound methods rely on the assumption 
Chapter 10. Non-invasive quantification of corneal biomechanical properties using OCT-Vibrography

that the corneal acoustic impedance is correlated with corneal elasticity, which has been demonstrated for low strain levels only [85]. Similarly, Brillouin microscopy relies on the relationship between the Brillouin and Young's modulus, which has been proved experimentally against rheology measurements [176]. Further experimental developments for OCT vibrography should include comprehensive in vitro evaluations in mounted whole eyes under different treatments, and improvements towards in vivo configuration, such as the use of a focused sound wave exciting the cornea to avoid the discomfort of high sound volume during measurements, and a lower corneal laser irradiance. An additional potential effect on the frequency response function in vivo may arise from the ocular muscles. This factor can be addressed computationally by modeling of ocular muscle damping, and experimentally by comparing in vivo with in vitro samples. 


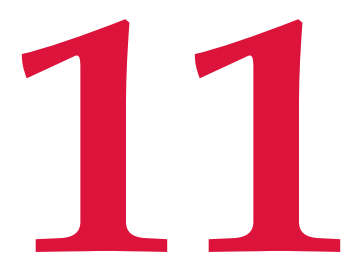

\section{Finite-Element Modeling of} Intrastromal Ring Segment Implantation into a Hyperelastic

Cornea

The outcome after Intrastromal Ring Segment Implantation varies highly across patients, probably because ICRS dimensions and its position are chosen according to empirical nomograms. In this study we build a numerical model to predict the results after ICRS implantation analyzing the effect of different factors.

This chapter is based on the publication by S. Kling and S. Marcos, 
Chapter 11. Finite-Element Modeling of Intrastromal Ring Segment Implantation into a Hyperelastic Cornea

entitled "Finite Element Modeling of Intrastromal Ring Segment Implantation into a Hyperelastic Cornea" in Investigative Ophthalmology and Vision Science.

The author of this thesis (i) designed the study, (ii) performed the finite element simulation and (iii) prepared the manuscript in collaboration with Susana Marcos. 


\section{Introduction}

Intrastromal corneal-ring segments (ICRSs) are currently used as a refractive technique to correct moderate to high myopia[36, 181] and as a therapeutic tool to improve the regularity of the aberrated corneal shape in astigmatic and keratoconic eyes.[171] In the latter, ICRSs also stabilize the weakened cornea. The first implants of peripheral intracorneal rings in humans were reported in 1966.[15] Later advances included changes in ICRS geometry (different arc lengths and heights, adjustable rings[64, 188]) and materials (synthetic gels[188, 192] or rigid materials[190]). Today, the most frequently implanted ICRSs are made of poly(methyl methacrylate) (PMMA), have a triangular ("Keraring"; Mediophacos Luda, and "Ferrara"; Ferrara Ophthalmics, both Belo Horizonte, Brazil) or hexagonal ("Intacs"; Addition Technology, Des Plaines, IL) shape and are available in several dimensions. For implantation, a tunnel parallel to the cornea is created at $70 \%-80 \%$ corneal depth,[118] in which the ICRS is subsequently inserted. Clinical studies report curvature changes of up to -13.75 diopters (D) after ICRS implantation,[189] as well as a small rotation of the ICRS.[36, 147] Clinically, the ICRS height and optical zone are modified to modulate the desired refractive change, typically from empirical nomograms. To our knowledge only a few analytical[43, 64, 153, 191] and one numerical study (Pinsky PM, et al. IOVS 1995;36:ARVO Abstract S308) have been proposed to model the response of the cornea to the ICRS. The studies suggest a linear relation between refractive change and ICRS height (the higher the ICRS, the more effective) and optical zone (ICRS inner distance to the apex, the smaller the more effective). This relation is also reflected in the clinically applied nomograms.[160] Although the biomechanical mechanisms underlying ICRS implantation have been approached, modeling relies strongly on the mechanical properties assigned to the corneal tissue and assumptions within the model. Corneal biomechanical models have improved in sophistication, from a single-membrane model with linear elastic[161] 
Chapter 11. Finite-Element Modeling of Intrastromal Ring Segment Implantation into a Hyperelastic Cornea

or viscoelastic [74] material properties, to hyperelastic orthotropic shell models that account for the tissue's microstructure,[152] as well as solid viscoelastic (Kling S., EVER, 2011, Instituto de Óptica, CSIC) and nonlinear[5] corneal models. Recently, the finite-element model (FEM) has also been suggested to model keratoconic corneas.[170] The microstructure of corneal tissue is dominated by collagen fibers (mainly type I[139]) that provide the necessary stability to maintain the spherical shape of the cornea. Keratoconus is generally thought to be associated with a local loss of collagen fibril orientation.[40, 132] This results in a local decrease of corneal rigidity $[52,150]$ and leads to the deformation in form of a cone.[126] The cone is typically slightly displaced interiorly and temporally from the corneal apex,[150] resulting in increased corneal aberrations [10, 126] and a highly distorted vision.[163] Keratoconic corneas with central cones were reported to occur in approximately[218] $25 \%$ of the patients. Recently, new quantitative imaging techniques have allowed analyzing geometric changes of the cornea before and after ICRS implantation.[147] Advanced imaging also allows a better understanding of the biomechanical mechanisms occurring with ICRS implantation, as a better verification of the simulated results is possible. We have developed a solid hyperelastic FEM of the anterior eye segment and studied the effect of ICRS implantation as a function of ICRS geometry and implantation parameters, for both normal corneas and a simple keratoconic model, with an altered elasticity profile. The model will reveal which parameters are most important to achieve the attempted refractive corrections after ICRS surgery. This will give a better understanding of the biomechanical response of the cornea and, hence, a more accurate prediction of the postsurgical results. 


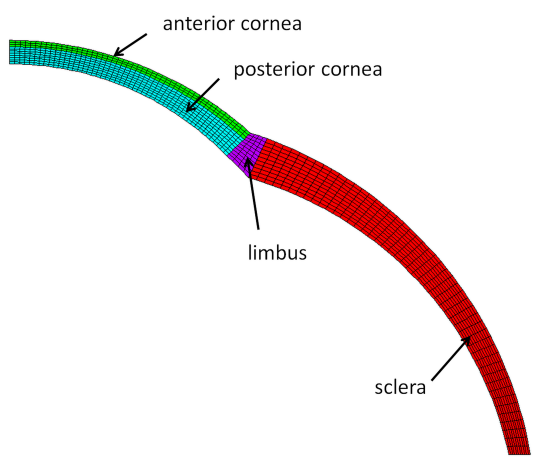

Figure 11.1: FEM geometry and mesh, consisting of anterior and posterior cornea, limbus, sclera.

\section{Protocols}

\section{Simulation of ICRS implantation}

A two-dimensional axis-symmetric model was built with a hyperelastic material for the ocular tissues. The geometry was defined by the cross-section of the anterior quarter of the outer eye coat, consisting of cornea, limbus, and sclera (see Figure 11.1). As previously found from experiments,[86] the anterior cornea was set slightly stiffer (factor 1.1) than the posterior cornea. Preoperative corneal curvatures (anterior, posterior) and thickness (538 $\mu \mathrm{m})$ were adjusted to represent an emmetropic eye with homogeneous corneal refraction of $42.2 \mathrm{D}$ over a $6-\mathrm{mm}$ pupil. The calculations were also performed for a simplified keratoconic eye with a centrally lower corneal elasticity. We applied a three-parameter Mooney Rivlin hyperelastic material model, similar to that previously proposed by Pandolfi et al.,18 with the following parameters: hyp1 = $1.500 \cdot 10^{3}$, hyp $2=1.415 \cdot 10^{5}$ hyp $3=2.139 \cdot 10^{7}, \mathrm{~d}=0$. The cornea was considered almost noncompressible[21] and thus Poisson's ratio was set to 0.5 . Corneal density[103] was set to $1062 \mathrm{~kg} / \mathrm{m}^{3}$. The sclera was modeled with a uniform Young's modulus of $33.6 \mathrm{MPa}$, set within the 
Chapter 11. Finite-Element Modeling of Intrastromal Ring Segment Implantation into a Hyperelastic Cornea

previously reported range (17-60 MPa).[73] The thickness variations along the sclera (from the limbus to the equator)were taken from the literature for pig eyes.[144] The scleral diameter was adjusted to human sclera $(23 \mathrm{~mm})$ and the limbus geometry was adjusted to match cornea and sclera, with material properties laying between both. A $15 \mathrm{~mm} \mathrm{Hg}$ pressure was applied to the posterior surfaces of cornea, limbus, and sclera, simulating physiologic intraocular pressure (IOP). Constraints were set at the scleral equator, permitting axial expansion to represent the natural eye fixation by the ocular muscles. A quadrilateral mesh was created for all geometric parts, resulting in approximately 1122 elements: 550 for cornea, 44 for limbus, and 528 for sclera (see Figure 11.1). The actual number of elements was dependent on the small variations in ICRS height, width, and corneal tunnel dimensions.

\section{FE Modeling of ICRSs}

ICRSs of triangular and hexagonal sections were modeled. Different ICRS heights and optical zones (defined as the distance from the inner ICRS edge to the corneal apex) were simulated, consistent with dimensions available on the market, including: 4.4, 5.0, 5.4, 6.0, and $6.6 \mathrm{~mm}$ optical zones; $150-350 \mu \mathrm{m}$ heights $(\mathrm{D}=50 \mu \mathrm{m})$, and $600 \mu \mathrm{m}$ width. The ICRS material was PMMA, an isotropic polymer with a Young modulus of 1800 MPa, a Poisson's ratio of 0.48 , and a density of $1170 \mathrm{~kg} / \mathrm{m} 3$.

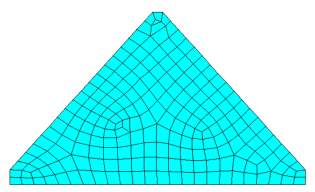

triangular ICRS

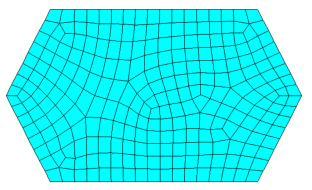

hexagonal ICRS

Figure 11.2: ICRS geometry and mesh of triangular and hexagonal crosssections (height: $350 \mu \mathrm{m}$ ). 


\section{Triangular Cross-Sectional ICRSs}

For the triangular ICRS the edges were slightly rounded, consistent with the actual shape of the ring, as reported from noncontact profilometric shapes of ICRS samples (Ferrara Rings and KeraRings; Pablo Perez-Merino and Susana Marcos, personal communication, 2009). A quadrilateral mesh was created for the ICRS consisting of 90 to 204 elements, depending on ICRS height. Triangular ICRSs are fabricated with a plane base; thus, the geometry was not further modified (see Figure 11.2A).

\section{Hexagonal Cross-Sectional ICRSs}

For the hexagonal ICRS the edges were set at $1 / 6$ width. The quadrilateral mesh consisted of 121 to 252 elements. Hexagonal ICRSs are fabricated with a tilted plane, adapted to the corneal curvature. This was implemented by rotating the ICRS by $-22^{c}$ irc (with respect to the position shown in Figure 11.2B) for implantation.

\section{Model Description}

For ICRS implantation three steps were considered: (1) Local material addition (ICRS), causing a local displacement of the corneal tissue. Friction between corneal tissue and ICRS was neglected. (2) Local stiffness increase due to PMMA insertion. (3) Applying only to triangular cross-sectional rings, disagreement between the orientation of the ICRS base with respect to the corneal tunnel, since the corneal tunnel is cut parallel to the corneal surface, but the ICRS is manufactured with a plane base.

\section{Simulation}

The simulation of the ICRS was performed following an incrustation method, using parameters similar to those used for clinical implantation. In a first step the tunnel was created at $75 \%$ depth of the cornea (oriented parallel to the corneal curvature) like that in surgery (70\%-80\%). 
Chapter 11. Finite-Element Modeling of Intrastromal Ring Segment Implantation into a Hyperelastic Cornea
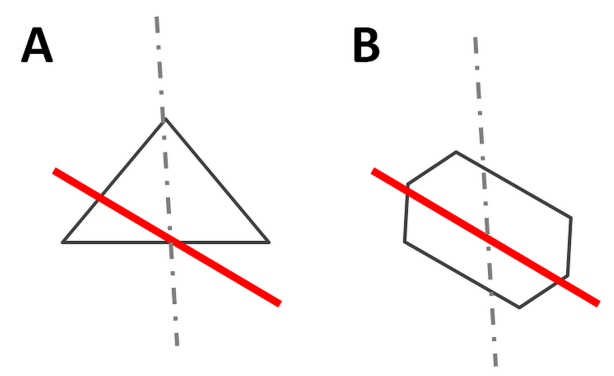

Figure 11.3: Positioning of the ICRS with respect to the tunnel (in red) within the cornea. (A) The triangular ICRS is positioned with the center of its base at the center of the tunnel. (B) The hexagonal ICRS is positioned with its center at the center of the tunnel.

Clinically, this is done either manually (separating collagen layers with a mechanical device) or with the use of a femtosecond laser. We simulated the tunnel by a theoretical gap between the upper and lower adjacent corneal areas, with a width slightly larger than that of the ICRS width. Although clinically the tunnel width is usually cut with a width similar to that of the ICRS, the corneal tunnel can be extended centrally toward the central visual axis or eccentrically toward the limbus (especially with manual tunnel creation).[38] A wider tunnel was also consistent with a more convergent simulation. The inner tunnel distance to the apex was set according to the optical zone of the ICRS. In a next step the ICRS geometry was placed within the cornea: for triangular ICRSs with the center of its base at the center of the tunnel; for hexagonal ICRSs with its center at the center of the tunnel (see Figure 11.3). Contacts without friction between the corneal tissue and the ICRS were simulated by flexible-flexible contact pairs[201] with a pinball region (contact search size) of 100 times the size of one finite element. Ramped effects of collision (i.e., linear increase of acting forces when establishing contacts) were defined between the ICRS surfaces and the tunnel surfaces. The response was analyzed after the ICRS had moved as an effect of establishing the contacts. Because viscoelastic material properties were not considered, 
the predicted deformed corneal shape describes the long-term effect of ICRS implantation.

\section{Keratoconic Model}

We studied a simplified keratoconic model consisting of an axis-symmetric cone. It is known from previous studies that keratoconic corneas exhibit a disorder of collagen fiber orientation at the location of the cone likely resulting in a local reduction of corneal tissue rigidity $[40,132]$ and resulting in a large local deformation. A hyperelastic material considered the nonlinear material properties of the corneal tissue with strain. In this study we investigated how local stiffness variations across the cornea affect the result of ICRS implantation. We simulated a pathologic cornea consisting of a central weakened zone, of different simulated diameters. Under the application of normal IOP (15 mm Hg) this led to the typical formation of a cone. Similar simple FEM models to mimic the local reduced elasticity modulus in keratoconus and its treatment by crosslinking have been recently presented by Roy and Dupps [170] Corneal weakening was implemented by reducing the three hyperelastic parameters (hyp 1-3) by a factor of $\mathrm{F}=100$ in an angular sector of the cornea (defined by the angle from the apex, at $90^{\circ} \mathrm{irc}$, toward the periphery, with the origin at the center of curvature of the cornea). Different cone extents were studied. Unless otherwise noted, the angular extent of the cone in keratoconic corneas was 78 (corresponding to a cone radius in the anterior cornea of $1.00 \mathrm{~mm}$ ). The amount of weakening was determined by reducing $\mathrm{F}$ until the IOP produced a deformation leading to a corneal power of $52.6 \mathrm{D}$ at the cone; this refraction corresponds to a stage II keratoconus in the Amsler-Krumeich scale. The deformation was consistent with a decrease in corneal thickness by $58.7 \mu \mathrm{m}$. Note, the tunnel was created before applying the IOP; thus, it will deform asymmetrically depending on the dimensions of the weakened tissue. 
Chapter 11. Finite-Element Modeling of Intrastromal Ring Segment Implantation into a Hyperelastic Cornea

\section{Results}

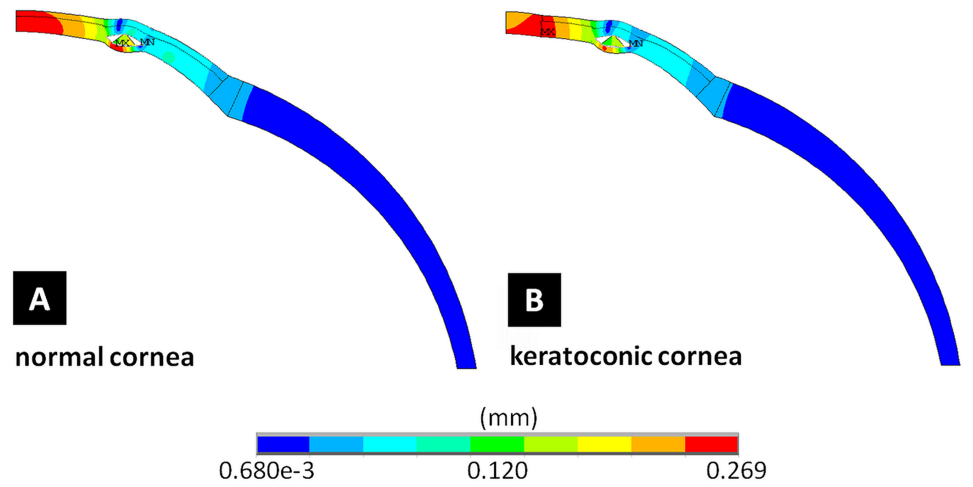

Figure 11.4: Absolute displacement after standard triangular ICRS (height: $300 \mu \mathrm{m}$; width: $600 \mu \mathrm{m}$; optical zone: 2.5 $\mathrm{mm}$ radius) implantation in a normal (A) and in a keratoconic cornea (B).

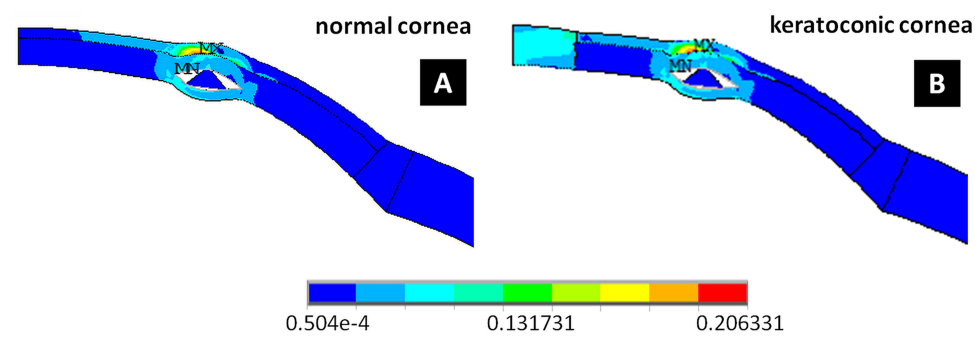

Figure 11.5: First principal strain distribution after standard triangular ICRS (height: $300 \mu \mathrm{m}$; width: $600 \mu \mathrm{m}$; optical zone: $2.5-\mathrm{mm}$ radius) implantation in a normal (A) and in a keratoconic cornea (B).

FEM predictions for the deformed corneal shape were analyzed for normal and keratoconic corneas. The absolute relative displacement of the surfaces and the first principal strain distribution resulting from the simulated implantation of a standard ICRS of triangular section (height: $250 \mu \mathrm{m}$; width: $600 \mu \mathrm{m}$; optical zone: $2.5 \mathrm{~mm}$ ) are shown 


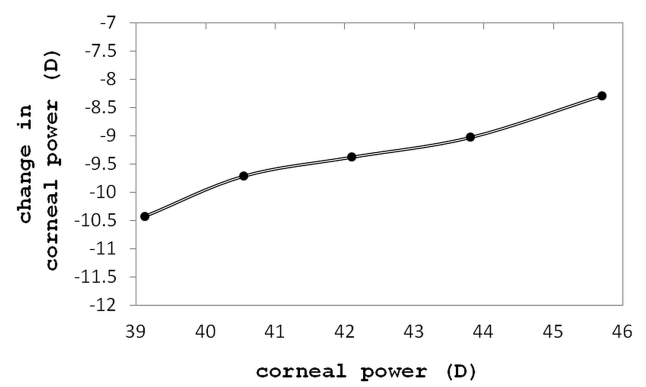

Figure 11.6: Changes in corneal power after standard triangular ICRS (height: $300 \mu \mathrm{m}$; width: $600 \mu \mathrm{m}$; optical zone: $2.5-\mathrm{mm}$ radius) implantation in a normal cornea as a function of the pre-op corneal power, analyzed for a pupil of 4-mm diameter.

in Figures 11.4 and 11.5, respectively. The anterior cornea showed a smooth transition near the location of the ICRS position, compared with the marked protrusion of the posterior cornea behind the ICRS. Although the geometric changes of the cornea were highly impacted by the geometry of the ICRS (see next subsections), preoperative corneal geometric parameters (such as the anterior corneal curvature) also played a role in the refractive changes after surgery. Figure 11.6 shows the effect of preoperative corneal curvature on refractive correction, with the higher corrections achieved with flatter corneas ( $-0.326 \mathrm{D}$ less refractive correction per $1 \mathrm{D}$ increase in curvature).

\section{Changes in Corneal Thickness}

Corneal thickness at the apex increased after ICRS implantation between 3.91 and $38.5 \mu \mathrm{m}$ for the normal cornea and between 66.8 and 97.9 $\mu \mathrm{m}$ for the keratoconic cornea, depending on ICRS geometry (see Figure 11.7). The higher ICRSs produced the largest increase in corneal thickness. Also hexagonal ICRS cross-sections increased central thickness less than that of triangular cross-sections. 
Chapter 11. Finite-Element Modeling of Intrastromal Ring Segment Implantation into a Hyperelastic Cornea

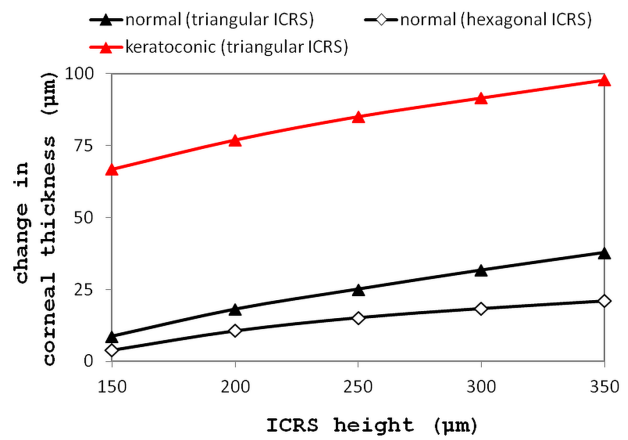

Figure 11.7: Changes in corneal apical thickness after ICRS (width $=600 \mu \mathrm{m}$ ) implantation in keratoconic and normal corneas, as a function of ICRS height $(150-350 \mu \mathrm{m})$; independent of optical zone dimensions. The simulation in the normal cornea is for triangular and hexagonal ICRSs, and for triangular ICRSs in the keratoconic cornea is for a triangular ICRS.

\section{Effect of ICRS Geometry on Anterior Corneal Curvature}

\section{Normal Corneas}

Figure 11.8 shows the effect of the ICRS on the change on the anterior corneal curvature (top row, Figure 11.8A,B) and the total corneal power (bottom row, Figure 11.8C,D), as a function of ICRS height, for different optical zone diameters $(4.4-6.6 \mathrm{~mm})$. Simulations were performed for triangular ICRS (left panels, Figure 11.8A,C) and hexagonal ICRSs (right panels, Figure 11.8B,D). Data are for a pupil of 4-mm diameter. The largest changes in anterior corneal curvature occurred for smaller optical zones (for a fixed ICRS height). ICRS with the largest optical zones (6 $\mathrm{mm}$ or larger) appeared to be ineffective in producing a change in curvature. Also, changes in anterior corneal curvature increased with ICRS height (for a fixed optical zone). Corneal power changed in a manner similar to that of the anterior corneal curvature, with slightly less negative refractive change, consistent with the positive contribution of the posterior surface. Hexagonal ICRSs produce lower changes than 

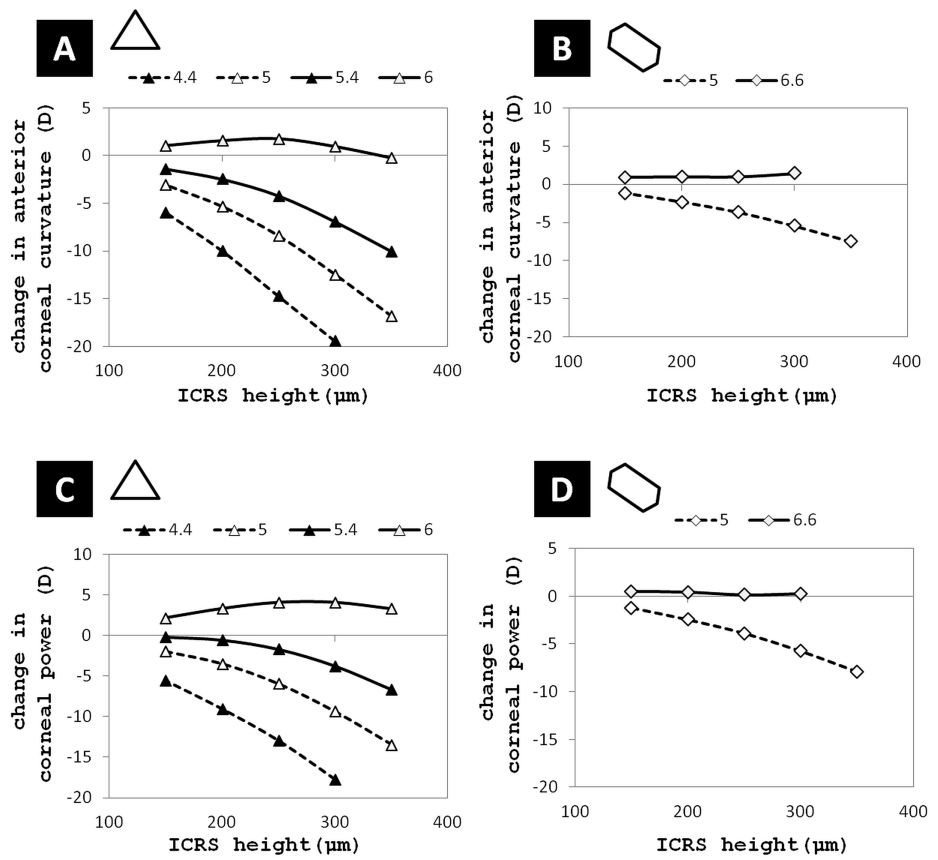

Figure 11.8: Normal cornea: changes in anterior corneal curvature $(A, B)$ and changes in corneal power $(C, D)$ after ICRS (width $=600 \mu \mathrm{m}$ ) implantation as a function of ICRS height for different optical zone diameters (4.4-6.6 $\mathrm{mm})$ and cross-sections ([A,C]: triangular; $[B, D]$ : hexagonal). Data are for 4-mm-diameter pupil. 
Chapter 11. Finite-Element Modeling of Intrastromal Ring Segment Implantation into a Hyperelastic Cornea

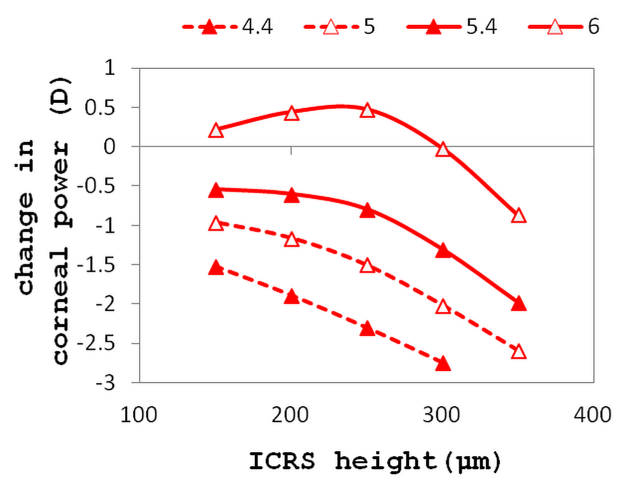

Figure 11.9: Keratoconic cornea: changes in corneal power after ICRS implantation (width $=600 \mathrm{~lm}$ ) as a function of ICRS height for different optical zone diameters (4.4-6.6 $\mathrm{mm}$ ). Data are for a 4-mm diameter pupil.

those produced by ICRSs with a triangular cross-section, but they also show an increase with ICRS height and a decrease with optical zone diameter.

\section{Keratoconic Corneas}

Figure 11.9 shows the effect of a triangular ICRS on corneal power on a keratoconic cornea. The curvature changes as a function of optical zone are similar to those found in normal corneas, whereas curvature changes as a function of ICRS height are slightly larger than those in normal corneas. Hexagonal ICRSs were not analyzed for keratoconic corneas, in that the triangular section ICRSs are more often used in clinics in the treatment of keratoconus. For a typical ICRS geometry used clinically (triangular section, $250 \mu \mathrm{m}$ height, 5-mm-diameter optical zone) our simulations predict a corneal power change close to $-10 \mathrm{D}$. 


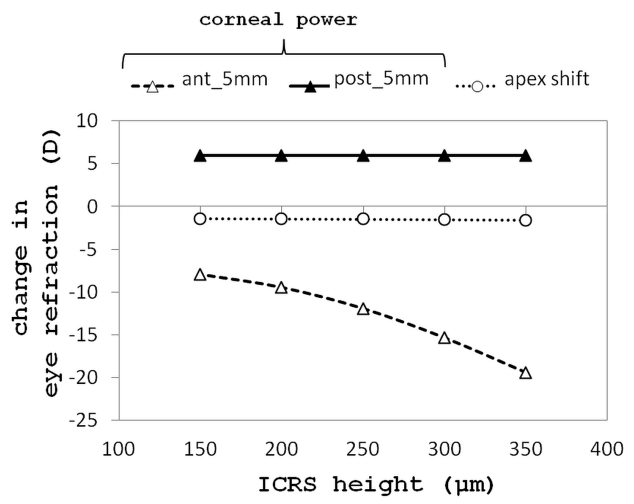

Figure 11.10: Contribution of the anterior and posterior corneal curvature and of the corneal apex shift to the absolute refractive change, after implantation of a triangular ICRS (width: $600 \mu \mathrm{m}$; 5-mm optical zone diameter) in a normal cornea. Data are for a 4-mm diameter pupil.

\section{Refractive Changes}

The changes in refraction are due to changes in curvature of both the anterior and posterior corneal surfaces, as well as changes in the relative position of the corneal apex. Figure 11.10 shows the relative contributions of the anterior and posterior corneal surfaces, and the relative shift of the apex (which ranges from $0.637 \mathrm{~mm}$ for the smallest optical zone and thinnest ICRS to $1.08 \mathrm{~mm}$ for the largest optical zone and highest ICRS) to the refractive change, after implanting a triangular 5-mm optical zone ICRS in a normal cornea. The anterior surface and the apex reduced corneal refraction, whereas the deformations of the posterior surface increased corneal refraction. On average, changes in the anterior cornea, posterior cornea, and apex shift contributed by $-75.4 \%$, $+12.3 \%$, and $-13.4 \%$, respectively, in a normal cornea. 
Chapter 11. Finite-Element Modeling of Intrastromal Ring Segment Implantation into a Hyperelastic Cornea
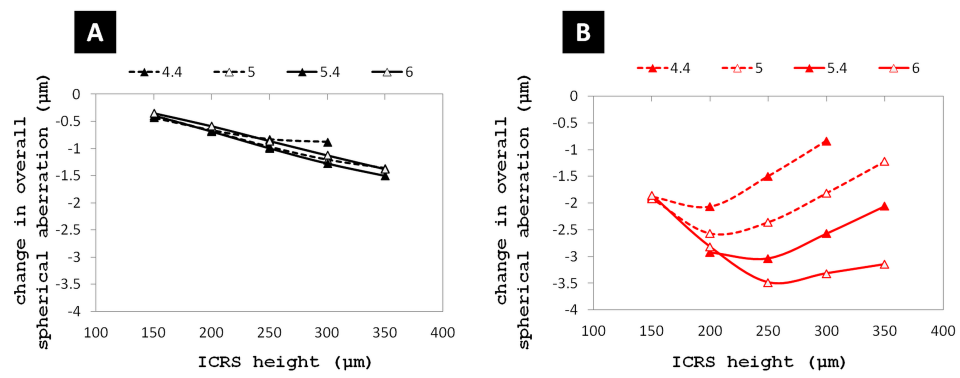

Figure 11.11: Changes of spherical aberration in a (A) normal and (B) keratoconic cornea after implanting triangular ICRSs (width $=600 \mathrm{~lm}$ ) as a function of ICRS height for different optical zone diameters (4.4-6 mm). Data are for 4-mm-diameter pupil.

\section{Changes in Spherical Aberration}

\section{Normal Corneas}

11.11A shows the change of the corneal spherical aberration (SA) for different optical zones, for a 4-mm-diameter pupil. SA was negative (consistent with a corneal prolate shape), its absolute value increased with ICRS height (between -0.5 and $-1.5 \mu \mathrm{m}$ ), and was stable across optical zones (in all cases larger than the pupil diameter under test).

\section{Keratoconic Corneas}

ICRSs implanted in a simulated keratoconic cornea also induced SA, with higher amounts in most cases than those in normal corneas (up to -3.5 $\mathrm{lm}$ ). Unlike in normal corneas, the largest absolute amount of SA was not induced with the highest ICRSs, but ICRSs with 200- $250 \mathrm{~lm}$ height (see Figure 11.11B). Also the induced SA varied with optical zone, with the larger optical zone inducing the largest amount of SA. 


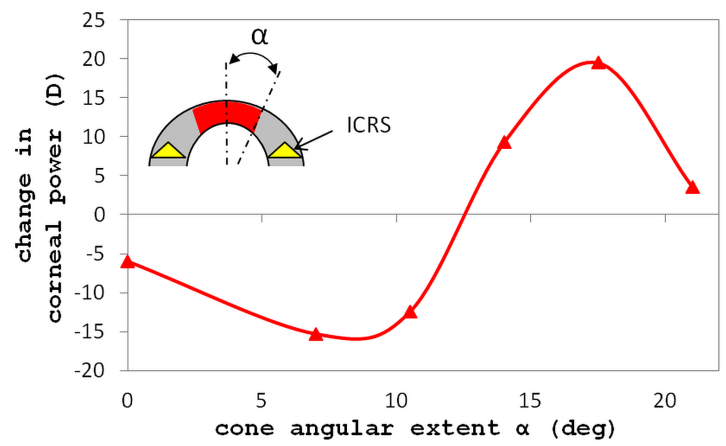

Figure 11.12: Changes in corneal power after standard triangular ICRS (height: $300 \mu \mathrm{m}$; width: $600 \mu \mathrm{m}$; optical zone: $5-\mathrm{mm}$ radius) implantation in a cornea with a central weakened zone of different angular extents $(\alpha)$ : $0^{\circ}$ (normal cornea) to $20^{\circ}$. The insets illustrate the definition of the angular dimensions of the cone.

\section{Effect of Keratoconus Size (Axis-Symmetric Model)}

The effect of ICRS implantation on corneal power was highly dependent on cone size. Figure 11.12 shows the effect of a standard triangular ICRS on the corneal reshaping of keratoconic corneas with weakened central area of different angular extents. The cornea was effectively flattened for cone extents smaller than $13^{\circ}$, but actually steepened for larger cones, with the corneal power change following a sinusoidal pattern as a function of cone size. The first point in the graph $\left(0^{\circ}\right)$ corresponds to a normal cornea. The induced spherical aberration also changed with cone size, reaching a maximum (absolute value) for a cone size of $17.5^{\circ}$.

\section{Discussion}

We present a finite-element model that identifies the most important parameters determining corneal deformation (and hence the change in refractive power) after ICRS implantation. Most previous predictions of ICRS-induced refractive changes were based on analytical,[43, 64, 
Chapter 11. Finite-Element Modeling of Intrastromal Ring Segment Implantation into a Hyperelastic Cornea

153, 191] numerical (Pinsky PM, et al. IOVS 1995;36:ARVO Abstract S308), or empirical regression models.[160] Fleming et al.[64] considered only the anterior corneal shape for estimations of the corneal refractive change. Although we can confirm that this is on average the largest factor $(75.4 \%)$, our calculations also show that the posterior surface $(11.3 \%)$ and the corneal apex shift (13.4\%) contribute significantly to the finally achieved refractive change after ICRS implantation. Although our model predicted the overall refractive outcomes found in patients, we observe slightly larger differences in the range of predicted refractive corrections (from 4.09 to $-17.71 \mathrm{D}$ in our model, for a 4-mm pupil) in comparison with clinical results (-1.0 to -6.0 D).[36, 181] Differences may arise from the assumed input biomechanical properties of cornea, limbus, and sclera, as well as from the geometric approximations in the model. We performed axis-symmetric simulation assuming an ideal spherical outer eye coat and a 3608 ICRS. Assuming an ideal spherical eye coat can be justified for myopic corrections in normal eyes. Also, an axis-symmetric model for keratoconus is representative of a centrally located keratoconus, which accounts for approximately $25 \%$ of the keratoconic population.29 Although a larger part of keratoconic corneas are asymmetric, the predictions of our symmetric model are consistent with clinical observations found in keratoconic patients implanted with ICRS: (1) ICRSs are more effective for smaller optical zones, (2) and there is an almost linear relationship between ICRS height and the amount of refractive decrease after implantation. We also found the triangular ICRS to be more effective than the hexagonal ICRS, which confirms previous clinical observations. [118] We found changes in corneal power of keratoconic corneas of 3.31 to $-20.5 \mathrm{D}$, which are consistent with keratometric reports from the literature ( 4.56 to -13.75 D).10 Also the predicted changes in spherical aberration $(-0.34$ to $-3.07 \mu \mathrm{m})$ from our simulations fall in the range of what is reported clinically $(-0.17$ to $-0.40 \mu \mathrm{m})$.[159] We observed an axial shift of the corneal apex after ICRS implantation (up to $-1.08 \mathrm{~mm}$ ). This effect has also been observed 
clinically, reporting a decrease in the anterior chamber depth after ICRS implantation.[135] As we could confirm in our simulations, the conical deformation in keratoconus makes the eye longer,[19] and the overall axial length of the eye decreases upon ICRS implantation. Our model also predicts the trend toward increased corneal thickness after ICRS implantation [147] as a result of material displacement toward the corneal apex, by 3.91 to $97.9 \mu \mathrm{m}$ (depending on the ICRS geometry and optical zone), which falls in the range of clinical observations (17 $\mu \mathrm{m},[62] 76$ $\mu \mathrm{m},[147])$. Due to the geometric shape of the ICRS, the central corneal thickness increase was more evident with triangular than with hexagonal cross-section (see Figure 11.7). In previous studies it was speculated that the ICRS rotates within the cornea, because its base is observed to be oriented with a certain angle respect to the corneal surface.[36, 147] Also an accumulation of cells at the ICRS edges has been observed using microscopy, [156] and tissue wound healing was attributed to induce the rotation. However, the disagreement between the orientation of ICRS base and corneal tunnel (triangular ICRSs only) is not a true rotation but rather a result from the plane shape of the rigid ICRS being inserted into the curved soft cornea. Additional smallest rotations moving the ICRS base away from its horizontal orientation may occur due to acting forces along the ICRS or the mentioned healing process. This effect is much smaller than the disagreement in orientation between the ICRS base and the corneal tunnel (5 to $12^{\circ}$, [189] vs. $45^{\circ}$ (angle between corneal surfaces and ICRS base)) and can also be observed in the simulations (see Figures 11.4 and 11.5). The biomechanical changes occurring with keratoconus are still largely unknown, although a decrease in ocular rigidity in keratoconic eyes has been reported.[19, 52] This probably results in a local stretching that would explain the decreased corneal thickness at the cone. We studied the effect of decreasing the hyperelastic parameters, which result in a decrease in corneal stiffness and a local change in curvature. Previous studies[72, 170] simulated the weakened keratoconic cornea by decreasing stiffness and corneal thickness sepa- 
Chapter 11. Finite-Element Modeling of Intrastromal Ring Segment Implantation into a Hyperelastic Cornea

rately. However, ideally, the change in mechanical parameters would cause the out-bulging and thinning at once. We captured this aspect in our model by decreasing corneal stiffness until the IOP led to the formation of a cone. Note that this weakening refers to the unloaded cornea. As known from stress-strain graphs, the elastic modulus of hyperelastic materials depends on the amount that the material has been stretched. We observe highest strains at the position of the cone, which causes a local hardening of the corneal tissue compared to its unloaded condition. From estimations from our model, the Young's modulus of the stressed keratoconic cornea is approximately 10 times weaker than the normal cornea. Experimentally, the keratoconic cornea is estimated to be $0[140]$ to 2.04[6] weaker than normal cornea. The fact that this is the average across keratocones of different severity might justify the need of stronger weakening for a $52.6 \mathrm{D}$ cone like that in our simulation. Our axis-symmetric keratoconic model described the geometric change-effect of implanting an ICRS with an altered elasticity profile and the corresponding conical deformation. This is accurate for centrally located keratocones and a good approximation for asymmetric keratocones, because the cone is always centered within the ICRS. Very interestingly, apart from the geometric parameters of the ICRS and the optical zone, the extent of the cone played a primary role in the effectiveness of the ICRS. The lack of effectiveness (and actual sign reversal in the corneal power range) for larger cones may explain, at least in part, the high outcome variability found clinically. $[118,160,189,190]$ A limitation of our model is the lack of conformity of the corneal tissue to the ICRS shape, unlike the evidence from in vivo OCT images in corneas implanted with ICRSs implanted in corneas.[147] Nevertheless, Twa and colleagues[210] observed new collagen formation, increased keratocyte density, and lipid accumulation adjacent to the ICRS after implantation. We suggest that these processes might fill the free space observed in our simulation. Interestingly, the local shape of the anterior and posterior cornea in front and behind the ICRS observed in recently published fully 
quantitative corneal OCT images of corneas implanted with ICRSs11 is very well predicted by the model. Our model relied on the assumption of corneal mechanical parameters available from the literature. However, these parameters (in particular the Young's modulus) varies up to two orders of magnitude.[5, 109, 123, 224, 226] The predictability of our simulations will benefit from an increased accurate knowledge of the mechanical parameters of individual patient corneas. Newly developed systems [23, 45, 72, 149, 174] give hope that in vivo measurement of corneal elasticity will be possible soon: (1) optical coherence tomography (OCT) [147, 149] allows accurate measurements of corneal and limbus geometry, which are essential input parameters to FE models. Furthermore, OCT is useful to evaluate the outcomes of the model. (2) Brillouin microscopy has allowed in vivo estimates of the local elasticity map across the cornea.[174] (3) Second-harmonic microscopy (so far only applicable in vitro due to high-level light exposures) allows imaging the collagen fiber organization in the intact cornea. [23, 137] (4) Dynamic corneal imaging in response to an airpuff response analysis allows gaining insights into dynamic corneal biomechanical properties in vivo.[45] Our model predicts the effects of ICRS geometry on the corneal biomechanical response, which will benefit from more refined models and customized corneal geometric and mechanical parameters. Besides, the model gives insights on some surgical aspects that could be modulated to improve the refractive outcomes. For example, the model predicts that the relative orientation of the ICRS within the cornea plays a role in the corneal reshaping. Also, preliminary simulations suggested an increased effect of ICRS, when the tunnel is cut with a diameter slightly smaller than the diameter of the ICRS. Finally, the 2D axis-symmetric model could be extended to 3D to allow modeling of very asymmetric corneas, frequent in keratoconus. In summary, finite-element simulation of ICRS implantation allows identifying important parameters that determine corneal reshaping and refractive outcomes. This model permits a more detailed prediction of the surgical outcomes than currently used estima- 
Chapter 11. Finite-Element Modeling of Intrastromal Ring Segment Implantation into a Hyperelastic Cornea

tions, and thus a more systematic selection of the most appropriate ICRS parameters as well as a better understanding of the mechanisms involved in the biomechanical corneal response to surgery. This approach will become especially important as biomechanical models of the cornea become more sophisticated and incorporate corneal biomechanical properties of individual patients from emerging quantitative imaging systems. 


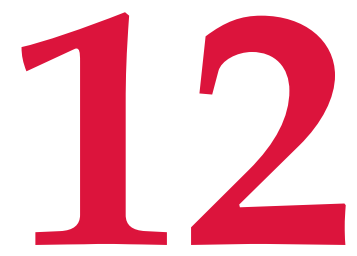

\section{Conclusions}

\section{Achievements}

- The stress-strain curve of the cornea in natural conditions and under the load of different intraocular pressures was assessed for cross-linked corneas and compared to virgin corneas for the first time.

- Storage media and the hydration state were identified to have a strong effect on the biomechanical response of corneal tissue.

- A new technique was developed for bi-directional flap extensiometry tests of corneal tissue, where stress is applied similar to the natural condition while corneal hydration can be controlled. 
- Brillouin microscopy was used to measure differences in the corneal stiffening pattern for different kinds of cross-linking and at different stages of the treatment.

- Geometrical parameters obtained from corneal deformation upon air-puff ejection were studied along with their dependency on corneal stiffness, corneal thickness, intraocular pressure and the presence of the sclera.

- A finite element model was built reproducing the experimental data on corneal deformation with air-puff at different hydration states, intraocular pressures and boundary conditions. The model was used to study the sensitivity of different geometrical deformation parameters with respect to their biomechanical properties.

- A novel vibration technique was developed to measure corneal dynamics by non-contact.

- Finite element simulations were performed to predict the refractive change after implanting intrastromal ring segments of different dimensions. The effect in healthy corneas was compared to keratoconic corneas. 


\section{Conclusions}

1.

Instillation of riboflavin-dextran solution reduced corneal thickness (by $281 \pm 5 \mu \mathrm{m})$. Cross-linking produced a 1.54-times reduction in corneal thinning and 2.8-times reduction in corneal apical rise with increased IOP. Anterior and posterior cornea flattened with increased IOP (less flattening in CXL eyes) and became steeper with decreased IOP. The horizontal meridian attened signicantly $(P<0.01)$ more than the vertical meridian. Young's modulus was higher in cross-linked eyes (1.096 \pm $\left.0.30 \frac{\mathrm{kN}}{\mathrm{m}^{2}}\right)$ than in non-crosslinked eyes $\left(0.692 \pm 0.30 \frac{\mathrm{kN}}{\mathrm{m}^{2}}\right)$. Hysteresis in non-treated eyes was also larger than in cross-linked eyes. Crosslinking stiffened porcine corneas signicantly. Both experimental data and stress-strain analysis are valuable for finite element models to improve understanding of CXL and its predictability. Although differences are expected between human corneas in vivo and porcine corneas ex vivo, the results are consistent with clinical data found in patients. The apparent biomechanical anisotropy of pig corneas must be confirmed in humans.

\section{2.}

Corneal deformation differed across conditions and hydration states. Dehydration by any dextran solution increased the hysteresis after the inflation/deflation cycle (14.29 vs 22.07 to $41.75 \mu \mathrm{m}$ ), whereas overnight hydration did not lead to a significant difference. Compared to control corneas, corneas treated with Optisol-GS showed the most similar behavior. Corneas treated with $0.125 \%$ riboflavin-20\% dextran deformed most ( $\Delta$ thickness $\left._{\max }=38.27 \mu \mathrm{m}\right)$, indicating a softening of the corneal tissue compared to control corneas $(23.18 \mu \mathrm{m})$ and corneas treated with $8 \%$ dextran $(21.01 \mu \mathrm{m})$ and $20 \%$ dextran $(29.07 \mu \mathrm{m})$. Dextran instillation decreased corneal thickness on average to $56.5 \%$ at 0 hours and $72.7 \%$ at 24 hours. Corneal hydration and tissue preservation changed corneal 
biomechanics, in particular its relaxation over a period of 24 hours.

3.

Flap curvature changed with increased function of IOP in pig flaps $\left(23.4 \cdot 10^{-3} \mathrm{D} / \mathrm{mmHg}\right)$. In rabbit flaps curvature changed signicantly less in 1 month post CXL $(P=0.026)$ than in untreated corneas (17.0 vs. $6.36 \cdot 10^{-3} \mathrm{D} / \mathrm{mmHg}$ ). Young's modulus was 2.29 megapascals (MPa) in porcine corneas, $1.98 \mathrm{MPa}$ in untreated rabbit corneas, and 4.83 $\mathrm{MPa}$ in 1 month post CXL rabbit corneas. At the same time, highly reflective structures were observed in the rabbit midstroma after treatment. 2Dflap extensiometry allows estimating corneal elasticity in vitro. The measurements are spatially resolved in depth, minimize the effects of corneal hydration, and preserve the integrity of the cornea. The method proved the efficacy of CXL in increasing corneal rigidity after 1 month in rabbits.

4.

Brillouin corneal stiffness increased signicantly $(P<0.001)$ by epi-off and epi-on CXL. The increase of Brillouin modulus was depth-dependent, indicating that anterior stromal stiffening contributes the most to mechanical outcome. The increase of anterior Brillouin modulus was linearly proportional to the light dose $(R 2>0.98)$. Compared to the standard epi-off procedure, a typical epi-on procedure resulted in a third of stiffness increase in porcine corneas $(C S I=33)$. Brillouin microscopy allowed imaging and quantifying CXL-induced mechanical changes without contact in a depth-dependent manner at high spatial resolution. This technique may be useful to evaluate the mechanical outcomes of CXL procedures, to compare different cross-linking agents, and for real-time monitoring of CXL in clinical and experimental settings. 


\section{5.}

We have confirmed non-invasive that Riboflavin and UV-cross-linking induce changes in the corneal biomechanical properties. These measurements are a first step for the estimation of the biomechanical properties of corneal tissue, at an individual level and in vivo, to improve diagnosis and prognosis of diseases and treatments involving changes in the biomechanical properties of the cornea.

\section{6.}

Temporal and spatial deformation profiles were very sensitive to the IOP $(p<0.001)$. The sclera slightly affected the temporal symmetry, while the ocular muscles drastically changed the amount of viscoelastic recovery. CXL produced a significant $(\mathrm{p}=0.001)$ reduction of the cornea indentation (by a factor of 1.41), and a change in the temporal symmetry of the corneal deformation profile (by a factor of 1.65), indicating a change in the viscoelastic properties with treatment. Corneal deformation following an air-puff allows the measurement of dynamic properties, which are essential for the characterization of corneal biomechanics.

\section{7.}

The proposed finite element model could reproduce well experimental data of the corneal response to an air-puff. It showed a good adaptability to different intraocular pressures, boundaries and different stiffness. The sensitivity analysis revealed a strong correlation between the geometrical deformation and biomechanical parameters and insensibility to side factors such as intraocular pressure and corneal thickness. Our model opens the way to retrieve dynamic material properties from dynamic imaging. 
8.

Simulations showed that corneal vibration in flaps is sensitive to both geometrical and biomechanical parameters, while in whole globes it is primarily sensitive to corneal biomechanical parameters. Natural frequency shifts suggest that posterior corneal flaps were 0.8 times weaker than anterior flaps and cross-linked corneas 1.6 times stiffer than virgin corneas. Sensitivity analysis showed that natural frequencies of whole globes were nearly independent from corneal thickness and IOP within the physiological range. OCT vibrography is a promising non-invasive technique to measure corneal stiffness without biases from corneal thickness and IOP.

9.

Predictions of the corneal response to implanted Intracorneal ring segment implantation indicate that ICRS geometry (height and optical zone) had a significant influence on corneal power: changes from 4.08 to -17.7 diopters (D) (healthy)/3.31 to $-20.5 \mathrm{D}$ (keratoconic) were observed. Central corneal thickness was predicted to increase by up to $38.5 \mu \mathrm{mm}$ (healthy)/97.9 $\mu \mathrm{m}$ (keratoconic). Spherical aberration also changed upon ICRS implantation. The protrusion of the posterior cornea behind the rings was well predicted. The model confirmed the clinically reported trends on the effect of ring geometry. FEM is a powerful tool to study the corneal response to ICRS implantation. The combination of FEM with individual biomechanical properties and geometry of patients holds promise to increase the predictability of ICRS surgery. 


\section{Future Work}

Clinical usage of FE MOdELS The finite element model developed to analyze the corneal deformation following an air-puff might be incorporated in the Corvis device and be used in clinical practice soon. A current limitation is time-consuming optimization process, however this could be overcome by using neural networks or genetic algorithms.

In vivo MEASUREMENTS WITH OCT VIBROGRAPHY A prototype for a corneal OCT vibrography system has been presented. Future versions of the device will use a focused sound wave and a lower laser scanning beam intensity. This will allow the application in vivo, where studying the effect of the natural fixation by ocular muscles will be an important point.

In vivo MEASUREMENTS WITH BRILLOUIN MICROSCOPY A first version of an in vivo Brillouin microscopy already has been built by S.H. Yun's lab at the Wellman Center for Photomedicine (Massachusetts General Hospital) and is currently being used to measure the difference between healthy and keratoconic corneas. Studies in the near future will evaluate the effect of UV cross-linking in vivo and on the long run Brillouin microscopy might be used in clinics as a diagnostic tool for kertatoconus in an early stage.

INCRASE CXL EFFICIENCY Different techniques have been presented that allow measuring the corneal biomechanics more accurately. This will be important in finding out factors, procedures and photo-sensitizers that might improve the efficacy of UV cross-linking. 
Chapter 12. Conclusions

256 de 329 


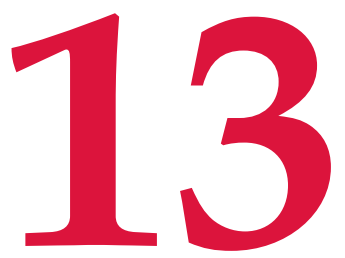

\section{Publications out of this thesis}

\section{Publications included in this thesis}

S. Kling, L. Remón, A. Pérez-Escudero, J. Merayo-Lloves, S. MARCos Corneal biomechanical changes after collagen cross-linking from porcine eye inflation experiments Investigative Ophthalmology and Vision Sciences [IF 3.597], 2010, 51(8): 3961-3968

C. Dorronsoro, D. Pascual, P. Pérez-Merino, S. Kling, S. Marcos Dynamic OCT measurement of corneal deformation by an air puff in normal and cross-linked corneas Biomedical Optics Express [IF 2.333], 2012, 3(3):473-487 
S. KLING, HS. GinIS, S. MARCOS Corneal biomechanical properties from two-dimensional corneal flap extensiometry: application to UV-Riboflavin cross-linking Investigative Ophthalmology and Vision Sciences [IF 3.597], 2012, 53(8): 5010-5015

S. Kling, S. MARcos Finite Element Modeling of Intrastromal Ring Segment Implantation into a Hyperelastic Cornea Investigative Ophthalmology and Vision Sciences [IF 3.597], 2013, 54(1): 881-889

G. Scarcelli, S. Kling, E. QuiJano, R. Pineda, S. Marcos, S.H. Yun Brillouin microscopy of collagen crosslinking: non-contact depth-dependent analysis of corneal elastic modulus Investigative Ophthalmology and Vision Sciences [IF 3.597], 2013, 54(2): 1418-1425

S. KLING, S. MARCOS Effect of hydration state and storage media on corneal biomechanical response from in-vitro inflation tests Journal of Refractive Surgery [IF 2.541], 2013, 29(7): 490-497

S. Kling, S. MARCos Contributing factors to corneal deformation in air-puff measurements Investigative Ophthalmology and Vision Sciences [IF 3.597], 2013, 54(7): 5078-5085

S. Kling, I.B. AKca, E. Chang, G. Scarcelli, N. BeKesi, S.H. Yun, S. MARCOS Quantifying OCT-Vibrography for the non-invasive estimation of corneal Biomechanical Properties in preparation

S. Kling, N. Bekesi, C. Dorronsoro, D. Pascual, S. Marcos Noninvasive in-vivo evaluation of corneal viscoelastic biomechanical parameters in preparation 


\section{Congress contributions}

S. Kling, L. Remón, A. Pérez-Escudero, J. Merayo-Lloves, S. MARcos Biomechanical response of normal and cross-linked porcine corneas ARVO, Fort Lauderdale (FL), 2009, Poster presentation

S. Kling, L. Remón, A. Pérez-Escudero, J. Merayo-Lloves, S. MarCOS Corneal biomechanical properties after cross-linking from quantitative imaging in an inflation porcine corneal model ASETCIRC (Asociación Española de Tecnología y Cirugía de Implantes, Refractiva y Córnea), Madrid (Spain), 2010, Invited oral presentation

S. Kling, L. Remón, A. Pérez-Escudero, J. Merayo-Lloves, S. MARcos Biomechanical effects in cross-linking Jornadas de Jóvenes Investigadores en Óptica Visual, Madrid (Spain), 2010, Oral presentation

S. Kling, P. PÉrez-Merino, S. Ortiz, D. Pascual, S. Marcos Corneal Biomechanical Response to Intraocular Pressure Changes From Scheimpflug and Anterior Segment OCT ARVO, Fort Lauderdale (FL), 2010, Poster presentation

S. Kling, P. PÉrez-Merino, S. Ortiz, D. Pascual, S. Marcos Corneal Biomechanical Properties From An Inflation Porcine Eye Model In Treated And Virgin Eyes Euromech 518 Workshop Biomechanics of the Eye, London (UK), 2010, Oral presentation

S. Kling, P. PÉrez-Merino, S. Ortiz, D. Pascual, S. Marcos Biomechanical Properties From Corneal Surface and Limbus Expansion upon Intraocular Pressure Variation from Optical Coherent Tomography and Finite Element Modeling Engineering the Eye III, Benasque (Spain), 2011, Poster presentation 
S. Kling, P. PÉrez-Merino, S. Ortiz, J.J. Del Coz Diaz, S. Marcos Corneal Biomechanical Characterization from In-Vitro Eye Inflation, Optical Coherence Tomography and Finite Element Modeling EVER, Crete (Greece), 2011, Oral presentation

S. KLING, H. Ginis, S. MARcos Corneal Biomechanical Properties and their Change with Corneal UV-Riboflavin Cross-linking from 2D FlapExtensiometry ARVO, Fort Lauderdale (FL, USA), 2012, Poster presentation

S. KLING, H. Ginis, S. Marcos Propiedades biomecánicas de la cornea determinadas por extensiometría bidimensional a partir de trazado de rayos. X Reunión Nacional de la Óptica, Zaragoza (Spain), 2012, Oral presentation

S. Kling, J.J. Del Coz DiAZ, J.L. SuAREZ, S. MARCos An orthotropic, viscoelastic model for the cornea and the effect of implanting an intrastromal ring segment. 6th European Congress on Computational Methods in Applied Sciences and Engineering (ECCOMAS), Vienna (Austria), 2012, Oral presentation

S. Kling, H. Ginis, G. Scarcelli, E. Chang, S.H. Yun, S. Marcos Spatially depth-resolved corneal elasticity: applications to cross-linking 8th International Congress of Corneal Crosslinking, Geneva (Switzerland), 2012, Invited oral presentation

S. Kling, D. Pascual, C. Dorronsoro, S. Marcos Corneal biomechanics: Deformation imaging with Air-puff 8th International Congress of Corneal Crosslinking, Geneva (Switzerland), 2012, Invited oral presentation

S. Kling, E.W. Chang, G. Scarcelli, S. Marcos, S.H. Yun OCTVibrography: A Novel Non-Contact Method to Estimate Corneal Biomechan- 
ical Properties ARVO, Seattle (WA, USA), 2013, Poster presentation

\section{Seminars}

S. KLING, S. MARCos Optical imaging to assess corneal biomechanics Grupo de señales, imágenes y ambientes virtuales, Universidad Autónoma de México, Mexico City (Mexico), 2013, Seminar 
Chapter 13. Publications out of this thesis 


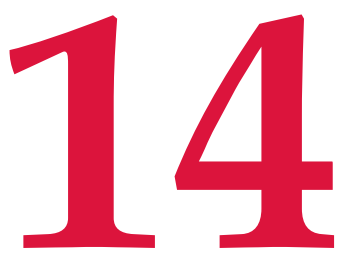

\section{Resúmenes en Español}

\section{Capítulo 1}

Este capítulo resume los antecedentes más relevantes en los campos de la fisiología, oftalmología, óptica y mecánica que han sido relevantes para el desarollo de esta tesis. En este capítulo se revisa la estructura biológica de las diferentes capas de la cornea, su refracción y los cambios con la edad. Después se define terminología del campo de la óptica utilizada durante la tesis, incluyendo la frente de onda, aberraciones, coherencia, interferencia y scattering de Brillouin. A continuación, se presentan brevemente las patologías del ojo (particularmente patologías corneales) y algunos tratamientos clínicos. Explicamos métodos numéricos para modelar la cornea y terminamos describiendo técnicas experimentales que se han aplicado para medir las propiedades biomecánicas de la 
cornea.

\section{Capítulo 2}

Este capítulo describe los métodos y materiales experimentales y teóricos que fueron utilizados en los capítulos siguientes. Primero se explican diferentes sistemas de imágen, incluyendo cámara de Scheimpflug, trazado de rayos, Tomografía de Coherencia Óptica y microscopía de Brillouin. Después se describen los aparatos y procedimientos para el manejo de ojos enucleados incluyendo un sistema de presión para ajustar la presión intraocular, una cámara húmeda que evita la deshidratación durante las medidas, soportes para montar los ojos delante los sistema y soluciones médicas y tratamientos aplicados para preservar el tejido, tal como para cambiar su rigidez. Seguimos con una descripción de rutinas para el análisis de diversos experimentos, incluyendo factores geométricos para describir la deformación de la cornea tras un pulso de aire, para determinar la deformación bidimensional en flaps a traves de coeficientes de Zernike, para determinar el modulo de elasticidad de la deformación geométrica durante el inflado del ojo entero e introducimos un factor para comparar diferencias en la rigidéz de la cornea de manera relativa. Terminamos el capítulo describiendo los bases de diferentes materiales en terminos de elementos finitos y explicamos como se analiza el cambio réfractivo en la geometría deformada obtenída de las simulaciones numéricas.

\section{Capítulo 3}

Es importante conocer la respuesta biomecánica de la cornea para mejorar los resultados de cirugías refractivas o tratamientos que alteran la rigidéz de la cornea. En este capítulo se comparan la respuesta de la córnea y cambios de la presión intraocular (IOP) en ojos no tratados y después de crosslinking (CXL). 
Se trataron 23 ojos enucleados con condiciones estándar de CXL (365 $\mathrm{nm}, 3 \mathrm{~mW}, 30$ minutos), y se tomaron 15 ojos contralaterales como control. Se tomaron imágenes tridimensionales con un Scheimpflug topógrafo de forma automática, mientras se mantuvo la presión intraocular constante (14 ojos) o variable (24 ojos, diferencia de $40 \mathrm{mmHg}$ en pasos de $4 \mathrm{mmHg}$ ). Los ojos se medieron dentro de una cámara húmeda (con control de temperatura y hídratación). Las medidas se realizaron inmediatamente después del tratamiento y se repetieron a las 24 horas después. Se analizó la geometría de la cornea en función de la IOP, y se estimaron las curvas de tensió y elongación para el globo ocular.

La aplicación de solución de riboflavina-dextrano redujó el espesor de la córnea (por $281 \pm 5 \mu \mathrm{m}$ ) y el tratamiento de CXL indució un adelgazamiento adicionalmente por un factor de 1.54. Aparte CXL disminuyó el desplacamiento del apex de la corneal en función de la presión intraocular por un factor de 2.8. Un aumento de la presión intraocular observó un aplanamiento de la cara anterior y posterior (que fue menor en ojos con CXL). El meridiano horizontal cambió significativamente $(P<0.01)$ más que el meridiano vertical. El módulo de Young fue mayor $\left(1.096 \pm 0.30 \mathrm{kN} / \mathrm{m}^{2}\right)$ en ojos con CXL que en los ojos control $\left(0.692 \pm 0.30 \mathrm{kN} / \mathrm{m}^{2}\right)$. Observamos un histéresis mayor en ojos no tratados.

Confirmamos que el tratamiento de CXL aumenta la rigidéz de las córneas de cerdo de manera significativa. Tanto los datos experimentales de deformació como las curvas de tensió y elongació son valiosa para modelos de elementos finitos y para una mejor comprensión de CXL y su predicció. Aunque se espera diferencias entre córneas humanas in vivo y córneas de cerdo ex vivo, los resultados son consistentes con los datos clínicos que se encuentran en pacientes. La aparente anisotropía biomecánica en ojos de cerdo debe ser confirmado en humanos. 


\section{Capítulo 4}

Evaluamos la influencia de la hidratación (modulada por soluciones de conservación/tratamiento y dependiente del tiempo post-mortem) sobre la deformacion de la cornea para distintas presiones intraoculares y la respuesta biomecanica.

Se usaron 30 ojos de cerdo recié enucleados y realizamos experimentos de inflación in vitro en los globos oculares enteros. Se dividieron los ojos en cinco grupos y se trataron con diferentes soluciones: $20 \%$ de dextrano, $8 \%$ de dextrano, 0.125\% riboflavin-20\% de dextrano, Optisol-GS, y un grupo de ojos virgenes (sin tratar) como control. Primero se aumentó la presión intraocular (de 15 a $55 \mathrm{mmHg}$ ) y después se disminuyó (a 15 $\mathrm{mmHg}$ ) en pasos de $5 \mathrm{mmHg}$. En cada paso se tomamron las imágenes con un topógrafo de Scheimpflug. A las 24 horas se repetieron las medidas. Se analizaron cambios en el espesor corneal y en la curvatura en función de la presión intraocular.

Observamos que la cornea se deforma de manera distinta bajo diferentes condiciones y estados de hidratación. Soluciones con dextrano deshidrataron la cornea y aumentaron el histéresis después del cíclo de inflación (14.29 vs 22.07 a $41.75 \mu \mathrm{m}$ ). Por el contrario la hidratación que se observó después de $24 \mathrm{~h}$ no dio lugar a una diferencia significativa. En comparación con las córneas de control, las córneas tratadas con Optisol-GS mostraron un comportamiento muy similar. Las córneas tratadas con $0.125 \%$ ribofl avin-20\% dextrano se deformaron más $\left(\Delta_{t h}\right.$ $=38.27 \mu \mathrm{m}$ ), lo que indica un pérdida de rigidéz del tejido corneal comparado con córneas control $(23.18 \mu \mathrm{m})$, córneas tratadas con $8 \%$ de dextrano $(21.01 \mu \mathrm{m})$ y con $20 \%$ de dextrano $(29.07 \mu \mathrm{m})$. La aplicación de dextrano disminuyó el espesor de la cornea en promedio a $56.5 \%$ a las 0 horas y a $72.7 \%$ a las 24 horas.

La hidratación y la conservación del tejido corneal cambian la respuesta biomecánica, en particular, su relajación durante un período de 24 horas. 


\section{Capítulo 5}

En estudios previos se han medido las propiedades biomecánicas de la córnea por extensiometría unidimensional o por métodos de inflado. En este estudio se ha desarrollado una técnica nueva de extensiometría bidimensional de un flap de la cornea combinando las ventajas de ambos métodos y la aplicamos para medir el efecto de crosslinking (CXL).

Se montaron flaps del estroma anterior $(96 \mu \mathrm{m})$ de corneas desepitelizadas (13 cerdos / 8 conejo) en un soporte que consiste en una lente BK7, una retina reflectante, y dos cámaras (una lleno de riboflavina-dextrano, otro llena de aceite de silicona). Se estiró el flap dentro del soporte durante cinco ciclos de cambio de presión (0-30-30 $\mathrm{mmHg}$ ) en pasos de $5 \mathrm{mmHg}$. El flap se deformó cambiando su superficie de plana a esférica, lo que se monitorizo mediante la estimacion de las aberraciones de Zernike (termino de desenfoque) mediante un aberrometro de trazado de rayos. Se utilizaron flaps de cerdo para demostrar el funcionamiento del sistema, y flaps de conejo para demostrar el efécto de crosslinking tras tratamiento in vivo (realizado mediante los procedimientos clínicos estándar) con ojos contralaterales sin tratamiento de control. Nuestros resultados nos permitieron estimar el módulo de Young a partir del cambio refractivo (extensión) en función de la presión (tensión). Para una mejor compresión de los resultados encontrados se examinaron las corneas de conejo en diferentes tiempos después de CXL.

Se obervaron cambios en la curvatura de los flaps en función de la presión aplicada (en cerdos $\left(23.4 \cdot 10^{-3} \mathrm{D} / \mathrm{mmHg}\right)$. En flaps de conejo la curvatura cambió significativamente menos 1 mes después de CXL ( $\mathrm{p}=$ 0.026 ) comparado con flaps no tratados (17.0 vs $6.36 \cdot 10^{-3} \mathrm{D} / \mathrm{mmHg}$ ). El módulo de Young fue 2.29 MPa en córneas de cerdo, $1.98 \mathrm{MPa}$ en córneas de conejos no tratadas y 4.83 MPa en córneas de conejo un mes después de CXL. Al mismo tiempo con el aumento de la rigidez se observaron estructuras reflectantes en el estroma intermedio en corneas de conejo.

El nuevo método de extensiometría bidimensional permitió la es- 
timación de la elasticidad de la córnea in vitro, la resolución de las propiedades a lo largo del espesor de la cornea, la reduccioón de los efectos de la hidratación y la preservación de la integridad dentro de la capa de la córnea. Se demostró el efecto de CXL para aumentar la rigidez en el estroma anterior después de 1 mes en conejos.

\section{Capítulo 6}

El tratamiento de crosslinking (CXL) está diseñado para aumentar la rigidez de la cornea y de esta manera impedir la progresión de queratocono o de ectasia. Todavia resulta difícil evaluar los resultados del tratamiento de manera cuántitativa debido a la falta de métodos adecuados de caracterizacion mecánica no invasiva. En este estudio se utilizós un sistema de microscopía de Brillouin para cuantificar el módulo de elasticidad a lo largo de la cornea antes y después de CXL.

El tratamiento de CXL se llevó a cabo en ojos frescos de cerdo. Se estudiaron los efectos de diferentes parámetros del tratamiento, como el tiempo de aplicación del photosensibilizador, el tiempo de iluminación con luz UVA y la presencia del epitelio. A partir de los mapas del módulo de Brillouin se analizaron las rigideces de la córnea en el estroma anterior, intermedio y posterior. Se introdujo un nuevo índice para comparar la rigidez de la cornea (CSI) haciendo referencia al aumento de rigidex observado despues de CXL estandar (aplicación de riboflavina por 30 minutos, iluminación con UVA de $3 \mathrm{~mW} / \mathrm{cm} 2$ durante 30 minutos).

El modulo de Brillouin de la córnea aumentó significativamente $(P<0.001)$ después de CXL, en los dos casos con y sin epitelio. El módulo de Brillouin demostró un gradiente a lo largo de la profundidad, lo que indica que el estroma anterior es la parte de la cornea que experimenta un cambio mayor de la rigidez con CXL. El aumento del módulo de Brillouin en el estroma anterior fue linealmente proporcional a la dosis de luz $(R 2>0.98)$. En comparación con el protocolo estándar, el protocolo sin epitelio resultó 33\% menos eficaz. 
La microscopía de Brillouin permitió visualizar y cuantificar los cambios mecánicos inducidos por el tratamiento de CXL de manera noinvasiva, sin contacto y con alta resolución espacial. Esta técnica se considera útil para evaluar los efectos mecánicos del tratamiento de CXL, sobre todo para comparar diferentes típos de CXL y para monitorizar en tiempo real la rigidé de la cornea clínicamente y experimentalmente.

\section{Capítulo 7}

Se presenta una nueva técnica para registrar la respuesta dinámica de la córnea tras un soplo de aire con un sistema de imágen. El sistema consiste de un OCT combinado con un tonómetro de aire para tomar imágenes de secciones transversales de la cornea entera y de la evolución temporal del apex. Se realizó un análisis cuantitativo que permitió extraer varios parámetros de deformación geométricos, como la indentación, el diámetro y el volumen de la deformación máxima, así como la duración y la velocidad de indentación y de recuperación. Se demostró el potencial de la técnica en córneas de cerdo in vitro bajo presión intraocular constante en diferentes condiciones (sin tratamiento, después de aplicar riboflavina y después de cross-linking), así como en córneas humanas in vivo.

La nueva técnica ha demostrado ser muy sensible para detectar diferencias en los parámetros de deformación a través de condiciones. Se confirmó de forma no invasiva que la riboflavina y cross-linking inducen cambios en las propiedades biomecánicas de la córnea. Estas diferencias parecen ser el resultado de cambios en las propiedades constituyentes de la córnea, y no es una consecuencia de los cambios en el espesor corneal, la geometría o la presión intraocular. Estas mediciones son un primer paso para la estimación de las propiedades biomecánicas del tejido de la córnea, a nivel individual e in vivo, para mejorar el diagnóstico y el pronóstico de las enfermedades y los tratamientos que implican cambios en las propiedades biomecánicas de la córnea. 


\section{Capítulo 8}

Recientemente se han presentado sistemas con pulso de aire para medir las propiedades biomecánicas de la córnea in vivo. En este estudio se evaluó el efecto de varios factores en la respuesta geométrica de la córnea a un pulso de aire: la presión intraocular (IOP), la rigidez de la córnea, la deshidratación, la presencia de la esclerótica, y la presencia de los musculos oculares.

Se utilizaron 14 ojos de cerdo recién enucleados, 5 ojos humanos donados para experimentos in vitro y 9 ojos humanos para experimentos in vivo. Se estudió la deformación de la cornea en función de: (I) la presión intraocular en el rango de 15 a $45 \mathrm{mmHg}$ (in vitro), (II) la deshidratación, después de aplicar riboflavina-dextrano (in vitro), (III) la rigidez de la cornea, después de aplicar el tratamiento de crosslinking (CXL) estándar (in vitro), (IV) las condiciones de contorno, como el efecto de la presencia de la esclerótica (comparación entre botones de la córnea y globos enteros, in vitro en cerdos) y (V) el efecto de los músculos oculares (comparación entre globos enteros humanos in vitro e in vivo). Se caracterizó la deformación temporal de la córnea por la indentación del apex a través del tiempo, la indentación máxima y la simetría temporal (comparando la deformación hacia el interior frente al exterior). El perfil de la córnea espacial se caracterizó por la distancia entre los dos picos en el estado de máxima deformación.

La deformación temporal y espacial fue muy sensible a la IOP ( $p<$ 0.001). La esclerótica afectó ligeramente la simetría temporal, mientras que los músculos oculares cambiaron drásticamente la cantidad de recuperación de la córnea (histéresis). El tratamiento de CXL redujó significativamente ( $p=0.001$ ) la indentación de la córnea (por un factor de 1.41), y cambió la simetría temporal (por un factor de 1.65), lo que indica un cambio en las propiedades viscoelásticas tras CXL.

El estudio de la deformación de la cornea tras un pulso de aire permitió medir las propiedades dinámicas, las cuales son esenciales 
para la caracterización de la biomecánica de la córnea.

\section{Capítulo 9}

Las propiedades biomecánicas son clave en el diagnóstico de patologías en tejidos biológicos, sin embargo su medida es difícil in vivo. Aunque se han propuesto diferentes métodos, todavía es necesario el desarollo de técnicas más precisas y menos invasivas. Recientemente se han desarrollado sistema que utilizan un pulso de aire para estudiar la dinámica de la córnea, de la piel y de colonias de bacterias. Típicamente se registra la deformación geométrica, que es relacionada indirectamente con los parámetros biomecánicos. Sin embargo, no existe un algoritmo sofisticado que relaciona los datos experimentales con las propiedades dinámicas intrínsicas de la muestra. En este estudio se presenta un modelo de elementos finitos del ojo capaz de reproducir los datos experimentales de la respuesta de la córnea a un pulso de aire. Se hicieron simulaciones para diferentes presiones intraoculares y condiciones de contorno y se mostró una buena adaptación del modelo a las diferentes condiciones. Debido a la importancia de las mediciones in vivo, se realizó un análisis de sensibilidad para esta condición con el fin de estudiar la correlación entre la deformación geométrica y los parámetros biomecánicos. Nuestros resultados muestran como imágenes dinámicas dan acceso a las propiedades intrínsicas e dinámicas de tejido.

\section{Capítulo 10}

Las propiedades biomecánicas de la cornea son clave para el diagnóstico de patologías y para la evaluación de tratamientos que alteran la refracció o la rigidez de la córnea. La mayoría de los métodos para medir la rigidez de la córnea son destructivos, mientras que los no destructivos que se aplican in vivo (por ejemplo midiendo la deformación de la cornea tras un pulso de aire) dependen mucho de la geometría (espesor) de la cornea 
y de la presión intraocular. En este estudio se demostró la capacidad de una nueva técnica, OCT vibrogrfía, para determinar los parámetros biomecánicos de la córnea, con menos limitaciones que en métodos previos.

Se realizarons simulaciones numéricas con un modelo de elementos finitos axisimétrico y se demostró la dependencia de la frecuencia natural de los parámetros biomecánicos de la cornea. Se aplicó un análisis modal y se estudió la respuesta de la oscilación en función de la frecuencia para flaps finos de la córnea y globos oculares enucleados. Se analizó el efecto de los siguientes parámetros: módulo de elasticidad, viscoelasticidad , la geometría (espesor, curvatura), la presión intraocular y la densidad. Se utilizó el modelo numérico para predecir la respuesta biomecánica en flaps de 3 córneas bovinas y en 2 ojos enucleados de cerdo. Para la comprobación experimental se excitó la cornea con sonido (100-110 db) y se utilizó un OCT sincronizado con la onda de sonido para medir la oscilación de la cornea en función de la frecuencia (en un rango de 50 a $510 \mathrm{~Hz}$ ). Las mediciones se llevaron a cabo en flaps de la corneal del estroma anterior y posterior, y en globos oculares enteros en dos condiciones (virgen y después de crosslinking) con el fin de estudiar el efecto de cambios en la rigidez de la córnea.

Los resultados de la simulación demuestran que la respuesta vibratoria de los flaps fue sensible tanto a parámetros geométricos como biomecánicos. En globos oculares enteros la respuesta vibratoria fue más sensible a los parámetros biomecánicos. El modelo predijo para una cornea más rígida frecuencias naturales más altas, lo cual se confirmó experimentalmente ( $\Delta f \sim 60 \mathrm{~Hz}$ después de CXL). Las frecuencias naturales están más espaciadas para los tejidos de menor rigidez, lo que sugiere que flaps de la cornea posterior son aproximadamente 0.9 veces menos rígidos que los flaps de la cornea anterior. La anchura de los picos de resonancia estuvo relacionada con la viscoelasticidad corneal. El análisis de la sensibilidad mostró que las frecuencias naturales son casi independientes del espesor de la córnea o de la presión intraocular 
(ambos dentro del rango fisiológico).

La vibrografía OCT es una técnica no invasiva prometedora para estimar las propiedades biomecánicas de la córnea, dado que permite aislar la rigidez de la córnea de otros parámetros. Las simulaciones permitieron determinar el rango de frecuencias de sonido necesario en los experimentos.

\section{Capítulo 11}

La cirugía de implante de segmentos de anillos intraestromales (ICRSs) se aplican para mejorar la visión en pacientes de queratocono, en miopes y en astigmatas. La selección de la geometría del ICRS y la posición de implantación se basan principalmente en nomogramas empíricos. En este estudio se desarrolló un modelo de elementos finitos (FEM) para mejorar la predicción post quirúrgica, estudiando la respuesta de la córnea a las diferentes geometrías del ICRS en córneas normal y con queratocono.

Se construyó un modelo de elementos finitos en dos dimensiones usando el software ANSYS-APDL. Se aplicó la teoría hiperelástica para los tejidos oculares (córnea, limbo, esclerótica) y la teoría elástica lineal para el ICRS de forma triangular o hexagonal hecho de poli metacrilato de metilo. Se desarrolló un modelo de incrustación que tiene en cuenta la adición de material local, el aumento de rigidez en la posición del ICRS y la diferencia geométrica de los anillos triangulares y hexagonales en su base (plano) con el túnel (paralelo a la superficie de la córnea). Se estudiaron diferentes alturas del ICRS (150-350 $\mu \mathrm{m})$ y zonas ópticas (4.4 a $6.6 \mathrm{~mm}$ ). El queratocono se simuló mediante un modelo axisimétrico con elasticidad de la córnea reducida a nivel local.

La geometría del ICRS (altura y zona óptica) afectó significativamente la refracción de la cornea: se observaron cambios de 4.08 a -17.7 D (sana) / 3.31 a -20.5 D (queratocono). El modelo también predice un aumento del espesor de la cornea central (38.5 $\mu \mathrm{mm}$ (sana) / $97.9 \mu \mathrm{m}$ (queratocono) y cambios en la aberración esférica. La protrusión de la 
córnea posterior detrás de los anillos coincide con datos experimentales. El modelo confirmó las tendencias reportadas clínicamente sobre el efecto de la geometría del anillo.

Un modelo de elementos finitos es una potente herramienta para el estudio de la respuesta de la córnea tras implantación de un ICRS. Usando propiedades biomecánicas y la geometría de pacientes individuales como datos de entrada en modelos de elementos finitos promete facilitar la predicción de cirugías de ICRS en el futuro.

\section{Conclusiones en Español}

\section{1.}

El tratamiento de cross-linking disminuye el espesor por un factor $1.54 \mathrm{y}$ reduce el desplazamiento del apex al aumentar la presión intraocular por un factor de 2.8 comparado con ojos control. La instilación de solución de riboflavina-dextrano reduce el espesor de la cornea $281 \pm 5 \mu \mathrm{m}$. La curvatura de la córnea anterior y posterior se aplana al aumentar la IOP (menos aplanamiento en los ojos CXL). El meridiano horizontal se aplana significativamente $(P<0.01)$ más que el meridiano vertical . El módulo de Young es mayor en ojos tratados mediante cross-linking (1.096 \pm 0.30 $\left.\frac{k N}{m^{2}}\right)$ que en los ojos control $\left(0.692 \pm 0.30 \frac{\mathrm{kN}}{\mathrm{m}^{2}}\right)$. La histéresis después de un ciclo de presión esayor en ojos control que en ojos con cross-linking. El tratamiento de cross-linking aumenta la rigidez de las córneas de cerdo de manera significativa. Tanto los datos experimentales como el análisis de tensión y extensión son valiosos para modelos de elementos finitos y para mejorar la comprensión del efecto de cross-linking y su predictibilidad. Aunque son probables ciertas diferencias entre córneas in vivo en humanos y corneas ex vivo en cerdos, los resultados experimentales son consistentes con datos clínicos en pacientes. La anisotropía en los dos meridianos de las corneas de cerdo debe ser confirmada en humanos. 


\section{2.}

Observamos que la córnea se deforma significativamente diferente dependiendo de su estados de hidratación y de las soluciones aplicadas. Cualquier solución con dextrano produce una deshidratación y aumenta la histéresis después del ciclo de presión (14.29 vs 22.07 a $41.75 \mu \mathrm{m}$ ), mientras que la hidratación durante la noche no da lugar a una diferencia significativa. En comparación con las córneas control, las córneas tratadas con Optisol GS muestran el comportamiento más parecido. Las córneas tratadas con $0.125 \%$ riboflavin-20\%dextrano se extienden más ( $\Delta$ thickness $s_{\max }=38.27 \mu \mathrm{m}$ ), lo que indica un ablandamiento del tejido de la córnea en comparación con córneas control $(23.18 \mu \mathrm{m})$, córneas tratadas con $8 \%$ de dextrano $(21.01 \mu \mathrm{m})$ o $20 \%$ de dextrano $(29.07 \mu \mathrm{m})$. La instilación de dextrano reduce el espesor de la córnea en promedio a $56.5 \%$ a 0 horas y a $72.7 \%$ a 24 horas. La hidratación de la cornea y su conservación cambian la respuesta biomecánica, en particular su relajación.

\section{3.}

La curvatura de flaps de cerdo aumenta al incrementar la presión dentro de la camara $\left(23.4 \cdot 10^{-3} \mathrm{D} / \mathrm{mmHg}\right)$. En flaps de conejo la curvatura cambia significativamente menos 1 mes después de CXL $(P=0.026)$ que en córneas no tratadas (17.0 dioptrías vs $6.36 \cdot 10^{-3} \mathrm{D} / \mathrm{mmHg}$ ). El módulo de Young es 2.29 MPa en córneas de cerdo, 1.98 MPa en córneas de conejos no tratadas, y 4.83 MPa en córneas de conejos 1 mes después de CXL. Al mismo tiempo que el aumento de rigidez después CXL observamos estructuras altamente reflectantes en el midstroma del conejo. La extensiometría bidimensional en flaps permite estimar la elasticidad de la corneal in vitro. Las mediciones estan resueltas espacialmente en profundidad, minimizan los efectos de la hidratación de la cornea, y preservan la integridad bidimensional de la córnea. El método demuestra el efecto de CXL para aumentar la rigidez en flaps 
corneales 1 mes después del tratamiento.

\section{4.}

El módulo de Brillouin de la corneal aumenta significativamente $(\mathrm{P}<$ 0.001) después de CXL, con y sin epitelio. El aumento del módulo de Brillouin depende de la profundidad, lo que indica que el aumento de rigidez en el estroma anterior contribuye más al efecto mecánico observado. El aumento del módulo de Brillouin es linealmente proporcional a la dosis de luz $(R 2>0.98)$. En comparación con el procedimiento estándar epi-off, el procedimiento epi-on resulta solo en un tercio del incremento de la rigidez en córneas porcinas $(C S I=33)$. La microscopía de Brillouin permite visualizar y cuantificar cambios mecánicos inducidos por CXL de manera sin contacto, en función la profundidad y con alta resolución espacial. Esta técnica puede ser útil para evaluar los resultados de los procedimientos mecánicos con CXL, para comparar los diferentes agentes de CXL, y para monitorizar en tiempo real el tratamiento de CXL en entornos clínicos y experimentales .

5.

Hemos confirmado de forma no invasiva que la riboflavina en combinación con luz UV induce cambios en las propiedades biomecánicas de la córnea. Estas mediciones son un primer paso para la estimación de las propiedades biomecánicas del tejido de la córnea a nivel individual e in vivo, para mejorar el diagnóstico y el pronóstico de enfermedades y tratamientos que implican cambios en las propiedades biomecánicas de la córnea.

\section{6.}

Los perfiles de deformación temporal y espacial son muy sensibles a la presión intraocular $(p<0.001)$. La esclerótica afecta ligeramente la 
simetría temporal, mientras que los músculos oculares cambian drásticamente la cantidad de recuperación viscoelástica. Cross-linking produce una reducción significativa $(\mathrm{p}=0.001$ ) de la indentación córneal (por un factor de 1.41) y un cambio en la simetría del perfil temporal de deformación (por un factor de 1.65), lo que indica un cambio en las propiedades viscoelásticas con el tratamiento. La deformación de la córnea tras un pulso de aire permite la medición de las propiedades dinámicas, que son esenciales para la caracterización de la biomecánica de la córnea.

\section{7.}

Presentamos un modelo de elementos finitos capaz de predecir los datos experimentales de la deformación corneal tras un pulso de aire. Mostramos una buena adaptación del modelo a diferentes presiones intraoculares, condiciones de contorno y diferente rigidez. El análisis de sensibilidad revela una fuerte correlación entre la deformación geométrica y los parámetros biomecánicos y demuestra la insensibilidad a factores laterales como la presión intraocular y el espesor córneal. Nuestro modelo abre el camino para obtener las propiedades dinámicas de tejidos a partir de imágenes de deformacón.

\section{8.}

Las simulaciones muestran que la vibración en flaps corneales es sensible tanto a los parámetros geométricos como biomecánicos, mientras que en globos enteros es principalmente sensible a los parámetros biomecánicos de la cornea. Cambios en las frecuencias naturales sugieren que flaps de la parte posterior de la cornea son 0.8 veces menos rigidos que los flaps de la parte anterior y que las córneas tratadas con cross-linking son 1.6 veces más rígidas que las córneas vírgenes. El análisis de sensibilidad muestra que las frecuencias naturales de globos enteros son casi independientes del espesor corneal y de la presión intraocular dentro del rango fisiológico. La vibrografía OCT es una técnica no invasiva 
prometedora que permite medir la rigidez de la cornea sin depender del espesor corneal y de la presión intraocular.

\section{9.}

Las simulaciones de implantación de anillos intracorneales en la córnea indican que la geometría del ICRS (altura y zona óptica) tienen una influencia significativa en la refracción corneal: observamos cambios entre 4.08 y -17.7 dioptrías en ojos sanos y entre 3.31 y $-20.5 \mathrm{D}$ en queratocono. El espesor corneal central aumenta hasta $38.5 \mu \mathrm{m}$ en ojos sanos y $97.9 \mu \mathrm{m}$ en queratocono. La aberración esférica cambia después de la implantación de ICRS. La simulación predice muy bien la protrusión de la córnea posterior detrás de los anillos. El modelo confirma las tendencias clínicas sobre el efecto de la geometría del anillo. FEM es una potente herramienta para estudiar la respuesta de la córnea para la implantación de ICRS. El método de FEM en combinación con propiedades biomecánicas individuales y geometrías individuales de pacientes promete aumentar la predictibilidad de la cirugía de ICRS . 


\section{List of Figures}

1.1 The eye, taken from Fuensanta et al [212]. . . . . . . . 2

1.2 Histology of the cornea indicating the five layers: $\mathrm{Ep}=$ epithelium, $\mathrm{Bo}=$ Bowman layer, $\mathrm{St}=$ Stroma, $\mathrm{De}=$ Descemet membrane, En=edothelium. (Taken from www.flapandzap.com) 3

1.3 Histology of the cornea showing keratocyte nuclei. (Taken from simple-med.blgspot.) . . . . . . . . . . . . 5

1.4 Left panel: basic shape of the first 15 Zernike polynomials; right panel: corresponding point-spread-functions, i.e. the way a punctual light source is imaged under the given aberration. Taken from [212]. . . . . . . . . 8

1.5 Principle of ray-tracing aberrometry. . . . . . . . . 12

1.6 Interference pattern, taken from www.goldberg.lbl.gov. . 13

1.7 Scheimpflug's principle. . . . . . . . . . . . . . . 14

1.8 Geometrical distortion observed in Scheimpflug images. 14

1.9 Scheimpflug image of the eye: (A) uncorrected, (B) corrected from distortions. . . . . . . . . . . . . . . 15

1.10 Typical image obtained from an ultrasonic bio-microscope. Here the focus is adjusted to the lens plane. The cornea appears deformed as optical distortion has not been corrected. The resolution is much lower compared to OCT images. . . . . . . . . . . . . . . . . 16

1.11 Differences in corneal orientation and the corresponding x-ray signal. Adapted from [136]. . . . . . . . . . . 18 
1.12 Differences in collagen orientation in a normal and keratoconic cornea. Adapted from [130]. . . . . . . . . . 18

1.13 Second harmonic generated images showing a higher collagen fiber branching in the anterior cornea compared to the posterior cornea. Adapted from [219]. . . . . . . 19

1.14 Intrastromal ring segments implanted in a human cornea. (Taken from [63]). . . . . . . . . . . . . . . 25

1.15 Stress-strain curves of a material showing three zones: (1) elastic deformation (Hooks law applies), (2) plastic deformation, (3) necking, with final rupture of the specimen .................... 36

1.16 Creep and relaxation testing scheme (typical for viscoelastic materials). . . . . . . . . . . . 38

1.17 1D corneal flap mounted within clamps for uni-axial stress-strain extensiometry measurements. . . . . . . . 40

1.18 Signal recorded with the Optical Response Analyzer (ORA), image taken from manufacturer website (www.reichert.com) 43

1.19 Corvis ST software. . . . . . . . . . . . . . 44

2.1 Commercial Pentacam Scheimpflug system. . . . . . . 52

2.2 Typical image of a human cornea obtained with the sOCT system. . . . . . . . . . . . . . . . . . 52

2.3 Sketch of the sOCT setup: SLD - superluminescent diode, OI - optical isolator, FC - 80:20 fiber coupler, PC polarization controller, NDF - neutral density filter, DC dispersion compensator, L1-L8 - lenses, $\mathrm{M}$ - silver mirror, PZT - piezotranslator, SC - galvanometric scanners, DM - dichroic mirror, $\mathrm{T}$ - target, HDG- holographic volume diffraction grating, CMOS - linescan camera, COMP computer. (Reproduced from [77]) . . . . . . . . . 53

2.4 sOCT system. . . . . . . . . . . . . . . . 54

2.5 Phase-sensitive swept-source OCT system. . . . . . . . 55

2.6 Commercial ray tracing system (iTrace). . . . . . . . . 56 
2.7 Set-up of the custom built sOCT air-puff system (bird's view). A tilted mirror is fixed to the tube tip of a modified non-contact air tonometer and provides an optical channel to a custom sOCT instrument (see text for details and angles). A custom eye holder and intraocular pressure control system (both in gray) were used for the in vitro experiments; in vivo experiments were performed without any further eye fixation. . . . . . . . . . 58

2.8 Patient view of the sOCT air-puff system. Air (blue) and laser (red) paths during the air-puff measurement by sOCT. 59

2.9 Set-up of the pressure control system. . . . . . . . . 61

2.10 Porcine eyes mounted within the wet chamber in order to prevent dehydration. . . . . . . . . . . . . . 62

2.11 Whole eye globe holder. . . . . . . . . . . . . 62

2.12 Corneal button holder. . . . . . . . . . . . . . 63

2.13 2D Flap holder. . . . . . . . . . . . . . . . 64

2.14 Carriazo-Pendular microkeratome. . . . . . . . . 65 
2.15 Diagram of the images resulting from the measurements and the corresponding extracted parameters. (a) Measurements of the temporal dynamics of the apex (Ascans) and (b) Measurements of the dynamics (B-scans) of a corneal meridian. 1: Corneal thickness. 2: Mean deformation speed during the increasing deformation period. 3: Peak speed (maximum slope) of the increasing deformation period. 4: Deformation amplitude (peak deformation, dividing the periods of increasing and decreasing deformation). 5: Peak speed of the decreasing deformation period. 6: Mean speed of the decreasing deformation period. 7: Duration of the increasing deformation period. 8: Duration of the decreasing deformation period. 9: overall duration of the deformation event. 10: Diameter of the deformed zone. 11: Displaced volume. 12: Non-deformed corneal radius. 13: Deformed corneal radius. See text for details. . . . . . . . . . . . . . . . 69

2.16 (A) Illustration of the corneal profile at maximal deformation and definition of the peak-to-peak distance (PDmax). (B) Illustration of the apex indentation as a function of time, and definition of the parameters of the temporal symmetry (Tsym), elastic air-puff response and the viscoelastic recovery and/or muscle damping. . . . . 71

3.1 Before Riboflavin-Dextran instillation . . . . . . . . . 86

$3.230 \mathrm{~min}$ after Riboflavin-Dextran instillation . . . . . . . . 86

3.3 Change in corneal thickness with IOP pressure, for crosslinked corneas $(\bullet)$ and non cross-linked corneas $(\Delta)$. Symbols are average data across eyes and the error bars stand for standard deviatons. A. Immediately after the treatment. B. 24-hours after the treatment. Data are average of $16 \mathrm{cxl}$ and 8 non-cxl. Error bars represent standard deviation. . . . . . . . . . . . . 88 
3.4 Apex shift as a function of (increasing and decreasing) pressure. Cross-linked corneas are represented by $\bullet$ and non-cross-linked by $\Delta$. Apex shift during 40 minutes (equivalent to duration of the pressure variation experiment) under constant pressure is shown in dashed line. Data are immediately after treatment. Data are average of $4 \mathrm{cxl}$ and 4 non-cxl. Error bars represent standard deviation. ............... 8

3.5 Change in anterior horizontal radius of curvature with varying pressure, immediately after (A) and 24-hours after treatment (B). Cross-linked corneas are represented by $\bullet$ and non cross-linked corneas by $\Delta$. Data are average of $16 \mathrm{cxl}$ and 8 non-cxl. Error bars represent standard deviation ..................... 91

3.6 Change in posterior horizontal radius of curvature with varying pressure, immediately after (A) and 24-hours after treatment (B). Cross-linked corneas are represented by $\bullet$ and non cross-linked corneas by $\Delta$. Data are average of $16 \mathrm{cxl}$ and 8 non-cxl. Error bars represent standard deviation. . . . . . . . . . . . . . 92

3.7 Change in anterior vertical radius of curvature with varying pressure, immediately after (A) and 24-hours after treatment (B). Cross-linked corneas are represented by $\bullet$ and non cross-linked corneas by $\Delta$. Data are average of $16 \mathrm{cxl}$ and 8 non-cxl. Error bars represent standard deviation.

3.8 Change in posterior vertical radius of curvature with varying pressure, immediately after (A) and 24-hours after treatment (B). Cross-linked corneas are represented by $\bullet$ and non cross-linked corneas by $\Delta$. Data are average of $16 \mathrm{cxl}$ and 8 non-cxl. Error bars represent standard deviation. . . . . . . . . . . . . 95 
3.9 Change in anterior radius of curvature (averaged horizontal and vertical) as a function of time, relative to the value at the beginning of the experiment. A. For variable pressure. B For constant pressure. The accompanying graphs represent the change of pressure with time, Cross-linked eyes are shown with $\bullet$ and non-crosslinked eyes with $\Delta$. Pressure variations induced a characteristic corneal deformation (much larger in untreated than cross-linked corneas). Cross-linking produced also a significant decrease in corneal curvature (even under constant pressure). . . . . . . . . . . . 96

3.10 Strain-stress curves derived from equations 1.41 and 2.3. • are for cross-linked corneas and $\Delta$ for untreated corneas. The left part of the curve corresponds to increased pressure and the right part of the curve to the decreased pressure. A. Immediately after treatment. B. After 24-hours. . . . . . . . . . . . 98

4.1 Initial corneal thickness before IOP variation for all tested conditions, at 0 hours and 24 hours. . . . . . . . . . 111

4.2 Corneal thickness response to IOP variation. Black represents $0 \mathrm{~h}$ data and grey $24 \mathrm{~h}$ data. IOP variation is respect to $15 \mathrm{mmHg}$ initial IOP. . . . . . . . . . . . . . 112

4.3 Changes in corneal radius of curvature as a function of IOP variation. Data are averaged of anterior and posterior changes, and $\mathrm{Oh}$ and $24 \mathrm{~h}$ measurements. . . . 113

5.1 Confocal microscopy images comparing the mid stroma (about $130 \mu \mathrm{m}$ depth) in three conditions (rabbits): (A) virgin cornea, (B) riboflavin instillation, (C) immediately post cxl, (D) one day post cxl, (E) one month post cxl. . 126 
5.2 Changes in defocus as a function of pressure on the flap in pig eyes (A) and rabbit eyes (B) pre and post-CXL (in vitro CXL in pigs and in vivo CXL in rabbits). . . . . . . . 127

5.3 Stress-strain diagrams in pig eyes (A) and rabbit eyes (B), pre and post-CXL (in vitro CXL in pigs and in vivo CXL in rabbits). The Young modulus was estimated as the slope of the linear regressions. . . . . . . . . . 128

6.1 Brillouin mechanical characterization of standard epi-off CXL procedure. (a) A representative cross-sectional Brillouin image of normal porcine cornea. (b) A Brillouin image of the cornea after the standard CXL. The horizontal and vertical span is $0.05 \mathrm{~mm}(\mathrm{x})$ by $0.8 \mathrm{~mm}(\mathrm{z})$ in a and b. (c) Brillouin depth profiles of both crosslinked and untreated corneas. (d) Mean Brillouin modulus of the anterior, mid-, and posterior regions for the crosslinked $(\mathrm{N}=6)$ vs. untreated corneas $(\mathrm{N}=3) .{ }^{*} * * P<0.005 \ldots 139$

6.2 The effects of the varying soaking time and light exposure time on the mean Brillouin modulus of the anterior, mid-, and posterior cornea. $* * P<0.01$; $* * * P<0.005 . \quad \ldots 140$

6.3 Mechanical outcome dependence on the light exposure time. (a) Mean Brillouin modulus of the anterior, mid, and posterior regions in the corneas treated with a presoaking time of 30 minutes and various UV exposure times of $0,5,15$ and 30 minutes, respectively. $* * P<$ 0.01 ; *** $P<0.005$. (b) The increase of mean Brillouin modulus in the anterior region as a function of exposure time. Circles, data; Error bars, standard deviations; Line, linear curve fit; . . . . . . . . . . . . . . . . . . 142 
6.4 Brillouin mechanical characterization of transepithelial "epi-on" CXL. (a) A representative cross-sectional Brillouin image of normal porcine cornea. (b) A Brillouin image of the cornea after epi-on CXL. The horizontal and vertical span is $0.05 \mathrm{~mm}(\mathrm{x})$ by $0.8 \mathrm{~mm}(\mathrm{z})$ in a and b. (c) Brillouin depth profile of epi-on CXL vs. soaked but not illuminated control cornea. (d) Mean Brillouin modulus of the anterior, mid-, and posterior regions for epi-on crosslinked $(\mathrm{N}=2)$ vs. untreated corneas $(\mathrm{N}=2)$. $* P<0.05 ; * * * P<0.005$. . . . . . . . . . . . . . 143

6.5 Mechanical effect of the corneal hydration state. (a) Brillouin depth profile of epi-off CXL vs. sham control (non-illuminated area of the same cornea). Dashed line indicates the average profile of untreated controls. (b) Brillouin depth profile of epi-on CXL vs. sham control. Dashed line indicates the average profile of untreated controls. . . . . . . . . . . . . . . . . . 144

6.6 Shear modulus at $0.2 \mathrm{~Hz}$ of central corneal button (4 mm diameter). Buttons were resected and immersed in epioff (dextran-based) solution $(\mathrm{N}=6)$ vs. epi-on (salinebased) soaking $(\mathrm{N}=6)$ for $30 \mathrm{~min}$ before measurement. Error bars: S.E.M. ${ }^{*} P<0.05 . \ldots \ldots 146$

7.1 Examples of dynamic A-scan measurements on the same porcine eye in vitro, (a) before and (b) after treatments of Riboflavin and (c) UV-cross-linking (c). The vertical axis represents axial depth (with the anterior corneal surface down) and the horizontal axis represents time. . 158 
7.2 Horizontal sections of the cornea (B-scans), for three different conditions of the same in vitro porcine eye: (a) virgin eye with no treatment; (b) after instillation of Riboflavin; and (c) after UV-cross-linking. The stable non-deformed cornea (reference condition) is shown in red, and the deformed cornea is overlapped in green. . . 158

8.1 (A) Corneal apex deformation as a function of time (B) Corneal profile at maximal deformation. Different colors represent different IOPs, ranging from 15 to $45 \mathrm{mmHg}$. Data are for whole porcine eyes. Each point is the average of measurements in 5 eyes. Error bars stand for the standard deviation across eyes. . . . . . . . . 174

8.2 (A) Corneal apex deformation during the deformation event (IOP fixed at $15 \mathrm{~mm} \mathrm{Hg}$ ); (B) corneal profile peakto-peak at distance at maximum deformation (PDmax) as a function of IOP. Data are average across 5 whole globes each: virgin corneas (blue); Riboflavin-Dextrantreated corneas (yellow); cross-linked treated corneas (red). Each point is the average of measurements in five eyes. . . . . . . . . . . . . . . 175

8.3 Corneal apex deformation across time in in vitro porcine corneas at IOP $=15 \mathrm{mmHg}$ (whole globes versus corneal buttons). Differences reveal the effect of the sclera. (A) Virgin porcine corneas: Each point is the average of measurements in 9 eyes. (B) Cross-linked porcine corneas: Each point is the average of measurements in 5 eyes. . . . . . . . . . . . . . 176 
8.4 Corneal apex deformation across time in human globes at IOP $=15 \mathrm{mmHg}$ (in vitro versus in vivo). Each point is the average of measurements in nine eyes in vivo and five eyes in vitro, respectively. Differences reveal the effect of ocular muscles. . . . . . . . . . . . . . 177

9.1 Air-puff characterization. (a) Experimentally measured temporal air-puff profile; (b) Results from CFD simulation showing the air-puff as a function of apex indentation and location along the cornea (horizontal distance from the apex). . . . . . . . . . . . . . . 190

9.2 Corneal deformation in porcine eyes in vitro at different IOPs. Experimental data (dotted lines) and simulation results (continuous lines) for a cross-linked porcine cornea: (a) apex indentation as a function of time, (b) spatial profile at maximal deformation. . . . . . . . . 192

9.3 Schematic of the simulation procedure. (1) corneal geometric data from Scheimpflug images are used to define the model geometry. Inverse modeling is performed to account for the effect of applying the IOP; (2) the temporal pressure profile measured experimentally and the spatial pressure profile obtained from CFD simulation are applied to the cornea as a function of time, location and current deformed shape; (3) the finite element model is solved for the current parameter set; (4) the simulation results are compared to the experimentally measured deformation. A step-wise optimization approach is used to find the parameter set that leads to the most similar deformation. . . . . . . . . . . . 197 
9.4 Corneal deformation pattern in human eyes in vivo. Experimental data (dotted lines) and simulation results (continuous lines) for the human cornea: simulations show the condition with and without ocular muscle damping; experimental data show in-vivo deformations (measured in patients) and in-vitro deformations (measured as an enucleated whole globe). . . . . . . . . . 198

9.5 Sensitivity analysis for geometrical and biomechanical parameters. (a) for the maximal apex indentation, (b) for the maximal peak distance. . . . . . . . . . . 198

10.1 Predicted dependence of the change in 1st and 2nd natural frequencies with (a) flap diameter, (b) flap thickness, (c) flap density, and (d) flap stiffness, from a sensitivity analysis. . . . . . . . . . . . . . . . 213

10.2 Left panel: Experimentally measured mode shapes at different frequencies in a posterior bovine flap. Right panel: Corresponding harmonics of the fundamental mode in corneal flaps, predicted from simulations in the range from 220 to $750 \mathrm{~Hz}$. . . . . . . . . . . . . 214

10.3 Frequency response function of the (a) simulated anterior and posterior corneal flaps, experimentally measured (b) anterior flaps, and (c) posterior bovine flaps. . . . . . 215

10.4 Simulated frequency response functions of whole eye globes for different (a) corneal stiffness, (b) density, (c) thickness, (d) curvature, and (c) IOP values. Red arrows indicate changes in the frequency response function when varying the corresponding parameter. . . . . . . 216

10.5 Predicted dependency of the natural frequency (1st-4th) shifts on (a) corneal stiffness and (b) corneal density. . . 217 
10.6 Predicted shifts in the frequency response function of the whole globe upon variations in (a) diameter of the cornea; (b) diameter of the sclera; (c) biomechanical properties of the sclera. The legend in (a) and (b) indicates the factor by which the dimensions of the cornea and sclera are modified. The legend in (c) indicate the scleral stiffness. . . . . . . . . . . . . . . . 218

10.7 Harmonics of the fundamental mode in whole globes, predicted from simulation in the range from 50 to $510 \mathrm{~Hz} .220$

10.8 response function of the (a) simulated and (b) experimental (average of 2 eyes per condition) whole globes, virgin (in blue) and cross-linked (in red). . . . . . . . . 221

10.9 Predicted frequency response function in whole eye globes, for a normal cornea (black line) and for corneas with a localized reduction of corneal stiffness (green line), with the weakened area located (a) centrally $\left(70-90^{\circ}\right.$ ) or (b) in a peripheral ring $\left(50-70^{\circ}\right) . \ldots . \ldots 222$

11.1 FEM geometry and mesh, consisting of anterior and posterior cornea, limbus, sclera. . . . . . . . . . . 231

11.2 ICRS geometry and mesh of triangular and hexagonal crosssections (height: $350 \mu \mathrm{m}$ ). . . . . . . . . . . 232

11.3 Positioning of the ICRS with respect to the tunnel (in red) within the cornea. (A) The triangular ICRS is positioned with the center of its base at the center of the tunnel. (B) The hexagonal ICRS is positioned with its center at the center of the tunnel. . . . . . . . . . . . . . 234

11.4 Absolute displacement after standard triangular ICRS (height: $300 \mu \mathrm{m}$; width: $600 \mu \mathrm{m}$; optical zone: $2.5-\mathrm{mm}$ radius) implantation in a normal (A) and in a keratoconic cornea (B). . . . . . . . . . . . . . 236 
11.5 First principal strain distribution after standard triangular ICRS (height: $300 \mu \mathrm{m}$; width: $600 \mu \mathrm{m}$; optical zone: 2.5 -mm radius) implantation in a normal (A) and in a keratoconic cornea (B). . . . . . . . . . . . . . 236

11.6 Changes in corneal power after standard triangular ICRS (height: $300 \mu \mathrm{m}$; width: $600 \mu \mathrm{m}$; optical zone: $2.5-\mathrm{mm}$ radius) implantation in a normal cornea as a function of the pre-op corneal power, analyzed for a pupil of 4-mm diameter. . . . . . . . . . . . . . 237

11.7 Changes in corneal apical thickness after ICRS (width $=600 \mu \mathrm{m}$ ) implantation in keratoconic and normal corneas, as a function of ICRS height (150-350 $\mu \mathrm{m})$; independent of optical zone dimensions. The simulation in the normal cornea is for triangular and hexagonal ICRSs, and for triangular ICRSs in the keratoconic cornea is for a triangular ICRS. . . . . . . . . . . . . 238

11.8 Normal cornea: changes in anterior corneal curvature (A, B) and changes in corneal power (C, D) after ICRS (width $=600 \mu \mathrm{m}$ ) implantation as a function of ICRS height for different optical zone diameters (4.4-6.6 mm) and cross-sections ([A, C]: triangular; $[\mathrm{B}, \mathrm{D}]$ : hexagonal). Data are for 4-mm-diameter pupil. . . . . . . . . . . 239

11.9 Keratoconic cornea: changes in corneal power after ICRS implantation (width $=600 \mathrm{~lm}$ ) as a function of ICRS height for different optical zone diameters (4.4-6.6 mm). Data are for a 4-mm diameter pupil. . . . . . . . . . . 240

11.10 Contribution of the anterior and posterior corneal curvature and of the corneal apex shift to the absolute refractive change, after implantation of a triangular ICRS (width: $600 \mu \mathrm{m}$; 5-mm optical zone diameter) in a normal cornea. Data are for a 4-mm diameter pupil. . . . . 241 
11.11 Changes of spherical aberration in a (A) normal and (B) keratoconic cornea after implanting triangular ICRSs (width $=600 \mathrm{~lm}$ ) as a function of ICRS height for different optical zone diameters $(4.4-6 \mathrm{~mm})$. Data are for 4-mm-diameter pupil. . . . . . . . . . . . . . . 242

11.12 Changes in corneal power after standard triangular ICRS (height: $300 \mu \mathrm{m}$; width: $600 \mu \mathrm{m}$; optical zone: 5 -mm radius) implantation in a cornea with a central weakened zone of different angular extents $(\alpha): 0^{\circ}$ (normal cornea) to $20^{\circ}$. The insets illustrate the definition of the angular dimensions of the cone. . . . . . . . . . . . . 243

\section{List of Tables}

1.1 Zernike polynomials used to describe optical aberrations. $x$, stands for horizontal and $y$ for vertical. . . . . . . . 10

1.2 Amsler-Krumeich scale to classify keratoconus severity. . . 22

1.3 Comparison of different material models. . . . . . . . . . 29

1.4 Corneal Young's moduli reported in literature. . . . . . . . 46

4.1 Summary of the different conditions studied. . . . . . . 108

4.2 Corneal normalized thickness before measurements at $0 \mathrm{~h}$ and 24 h. . . . . . . . . . . . . . 110

6.1 Mechanical efficacy of various CXL procedure quantified by the defined Corneal Stiffening Index. $\mathrm{Rf}=$ RiboflavinDextran; BAC = benzalkonium chloride. . . . . . . . . 147 
8.1 Overview on the different data sets, treatment and conditions used in the experiments. CCT $=$ central corneal thickness. . . . . . . . . . . . . . . . . . . . . 169

8.2 Summary of experimental results. . . . . . . . . 173

9.1 Model parameters. The table lists the biomechanical and geometrical parameters used to simulate the human and pig corneal deformation. . . . . . . . . . . . . . . . . 192

9.2 Parameter gradients. The table shows the result of the sensitivity analysis. . . . . . . . . . . . . . . . . 194

10.1 Sensitivity gradient of geometrical and biomechanical parameters respect to different resonance peaks, analyzed in a range of $\pm 20 \%$. . . . . . . . . . . . . 223

10.2 Sensitivity gradient of geometrical and biomechanical parameters respect to different resonance peaks, analyzed in a range of $\pm 20 \% \ldots \ldots$. . . . . . . . . . . . . 224 
LIST OF TABLES

294 de 329 


\section{Bibliography}

[1] ABAD, J., AND PANesso, J. Corneal collagen cross-linking induced by uva and riboflavin. Techniques in Ophthalmology 6, 1 (2008), 8-12.

[2] Ahearne, M., Yang, Y., Then, K., And LiU, K. An indentation technique to characterize the mechanical and viscoelastic properties of human and porcine corneas. Ann Biomed Eng 35, 9 (2007), 1608-1616.

[3] Alonso-Caneiro, D., Karnowski, K., Kaluzny, B., KowalczyK, A., AND WoJTKowski, M. Assessment of corneal dynamics with high-speed swept source optical coherence tomography combined with an air-puff system. Optics Express 19, 15 (2011), 1418814199.

[4] Ambrosio Jr, R., Nogueira, L., Caldas, D., Fontes, B., Luz, A., Cazal, J., Alves, M., And Belin, M. Evaluation of corneal shape and biomechanics before lasik. Int. Ophthalmol. Clin. 51, 2 (2011), 11-38.

[5] Anderson, K., El-Sheikh, A., And Newson, T. Application of structural analysis to the mechanical behaviour of the cornea. $J R$ Soc Interface 1 (2004), 3-15.

[6] Andreassen, T., Simonsen, A., And Oxlund, H. Biomechanical properties of keratoconus and normal corneas. Exp Eye Res 31 (1980), 435-441. 
[7] Asejczyk-Widlicka, M., And Pierscionek, B. The elasticity and rigidity of the outer coats of the eye. Br J Ophthalmol 92, 10 (2008), 1415-1418.

[8] Avery, N., AND BAIley, A. Restraining Cross-Links Responsible for the Mechanical Properties of Collagen Fibers: Natural and Artificial. Collagen - Sturcture and Mechanics, Fratzl P., Springer, 2008.

[9] BALDWin, W., AND MilLs, D. A longitudinal study of corneal astigmatism and total astigmatism. American Journal of Optometry adn Physiologic Optics 58, 3 (1981), 206-211.

[10] Barbero, S., Marcos, S., Merayo-Lloves, J., And MorenoBARRIUSO, E. Validation of the estimation of corneal aberrations from videokeratography in keratoconus. J Refract Surg 18 (2002), 263-270.

[11] BASHKAR, G. Photodynamic therapy (pdt). Resonance 5, 6 (2000), 15-29.

[12] BeEms, E., AND VAN-Best, J. Light transmission of the cornea in whole human eyes. Exp Eye Res 50, 4 (1990), 393-395.

[13] Beil, M., Micoulet, A., von Wichert, G., Paschke, S., Walther, P., OMARY, M., VAN Veldhoven, P., Gern, U., WolfFHieber, E., Eggermann, J., Waltenberger, J., Adler, G., SPATZ, J., AND SEUfFERLEIN, T. Sphingosylphosphorylcholine regulates keratin network architecture and visco-elastic properties of human cancer cells. Nature Cell Biology, 5 (2003), 803-811.

[14] Binder, P. Ectasia after laser in situ keratomileusis. J. Cataract Refract. Surg. 29, 12 (2003), 2419-2429.

[15] Blavatskaia, D. The use of intralamellar homoplasty in order to reduce refraction of the eye. Oftalmol Zh 7 (1966), 530-537. 
[16] Boyce, B., Grazier, J., Jones, R., And Nguyen, T. Fullfield deformation of bovine cornea under constrained inflation conditions. Biomateriales 29, 28 (2008), 3896-3904.

[17] Boyce, B., Jones, R., Nguyen, T., And Grazier, J. Stresscontrolled viscoelastic tensile response of bovine cornea. Journal of Biomechanics 40, 11 (2007), 2367-2376.

[18] Boyer, G., Pailler, M., Molimard, J., Pericoi, M., Laquieze, S., AND ZAHOUANI, H. Non contact method for in vivo assessment of skin mechanical properties for assessing effect of ageing. Medical Engineering Physics 34 (2012), 172-178.

[19] Brooks, A., Robertson, I., AND MAhoney, A.-M. Ocular rigidity and intraocular pressure in keratoconus. Aust J Ophthalmol 12 (1984), 317-324.

[20] Brouwer, I., Ustin, J., Bentlez, L., Sherman, A., Dhruv, N., AND Tendick, F. Measuring In Vivo Animal Soft Tissue Properties for Hatpic Modeling in Surgical Space, Inner Space, Virtual Space, vol. 81. Book Series: Studies in health technology and informatics, 2001.

[21] BRyant, M., AND McDonnell, P. Constitutive laws for biomechanical modeling of refractive surgery. J Biomech Eng 118 (1996), 473-481.

[22] Bueno, J., Gualda, E., AND ARTAl, P. Analysis of corneal stroma organization with wavefront optimized nonlinear mircroscopy. Cornea 30, 6 (2011), 692-701.

[23] Bueno, J., Gualda, E., Giakoumaki, A., Pérez-Merino, P., Marcos, S., AND ARTAL, P. Multiphoton microscopy of ex vivo corneas after collagen cross-linking. Invest Opthtalm Vis Sci 52, 8 (2011), 5325-5331. 
[24] Butcher, D., Alliston, T., And Weaver, V. A tense situation: forcing tumour progression. Nature Reviews Cancer, 9 (2009), 108-122.

[25] Cano, D., Barbero, S., And Marcos, S. Comparison of real and computer-simulated outcomes of lasik refractive surgery. JOSA A 21 (2004), 926-936.

[26] Caporossi, A., Baiocchi, S., Mazzotta, C., Traversi, C., And CAPOROSSI, T. Parasurgical therapy for keratoconus by rib oflavinultraviolet type a rays induced cross-linking of corneal collagen - preliminary refractive results in an italian study. Journal of Cataract and Refractive Surgery 32 (2006), 837-845.

[27] CAREW, E., BARber, J., AND Vesely, I. Role of preconditioning and recovery time in repeated testing of aortic valve tissues: Validation through quasilinear viscoelastic theory. Ann of Biomed Eng 28 (2000), 1093-1100.

[28] Celic, D., AND Boltezar, M. Identification of the dynamic properties of joints using frequency-response functions. Journal of sound andvibration 317 (2008), 158-174.

[29] Cennamo, G., Rosa, N., La Rana, A., Bianco, S., and SeBASTIANI, A. Non-contact tonometry in patients that underwent photorefractive keratectomy. Ophthalmologica 211, 6 (1997), 341-343.

[30] Chang, E., Kobler, J., And S.H., Y. Subnanometer optical coherence tomographic vibrography. Optics Letters 37, 17 (2012), 3678-3680.

[31] Chao, C., Zhen, Y., AND Cheing, G. A novel noncontact method to assess the biomechanical properties of wound tissue. Wound Repair and Regeneration 19, 3 (2011), 324-329. 
[32] Chen, S., Urban, M., Pislaru, C., Kinnick, R., Zheng, Y., YAO, A., AND GREENLEAF, J. Shearwave dispersion ultrasound vibrometry (sduv) for measuring tissue elasticity and viscosity. IEEE Trans Ultrason Ferroelectr Freq Control 56, 1 (2009), 55-62.

[33] Cheng, X., AND PInsky, P. Mechanisms of self-organization for the collagen fibril lattice in the human cornea. $J$ r Soc Interface 10, 87 (2013), 1742-5662.

[34] Choi, D., Thompson JR, R., And Price JR, F. Incisional refractive surgery. Curr. Opin. Ophthalmol. 13, 4 (2002), 237-241.

[35] Clough, R. W. The finite element method in plane stress analysis. Proc. American Society of Civil Engineers 23 (1960), 345-378.

[36] Cochener, B., Savary-LeFloch, G., and Colin, J. Effect of intrastromal corneal ring segment shift on clinical outcome: one year results for low myopia. J Cataract Refract Surg 26 (2000), 978-986.

[37] Colin, J., Cochener, B., Savary, G., And Malet, F. Correcting keratoconus with intracorneal rings. J Cataract Refr Surg 26, 8 (2000), 1117-1122.

[38] Coskunseven, E., Kymionis, G., And Jankov, M. Complications of intra corneal ring segment implantation with femtosecond laser channel creation in patients with keratoconus (explanations and solutions). J Emmetropia 1 (2010), 221-228.

[39] Courant, R. Variational methods for the solution of problems of equilibrium and vibrations. Bulletin of the American Mathematical Society 49 (1943), 1-23.

[40] Daxer, A., AND Fratzl, P. Collagen fibril orientation in the human corneal stroma and its implications in keratoconus. Invest Ophthalmol Vis Sci 38 (1997), 121-129. 
[41] Daxer, A., Misof, K., Grabner, B., Ettl, A., And Fratzl, P. Collagen fibrils in the human corneal stroma: Structure and aging. Invest Ophthalmol Vis Sci 39 (1998), 219-224.

[42] Deenadayalu, C., Mobasher, B., Rajan, S., And Hall, G. Refractive change induced by the lasik flap in a biomechanical finite element model. J Refract Surg 22, 3 (2006), 286-292.

[43] Duotyan, G., Kurtz, R., Fernandez, D., And Juhasz, T. An analytically solvable model for biomechanical response of the cornea to refractive surgery. J Biomech Eng 123 (2001), 440-445.

[44] Doors, M., TAhzib, N., Eggink, F., Berendschot, T., Webers, C., AND NUIJTs, R. Use of anterior segment optical coherence tomography to study corneal changes after collagen cross-linking. Am J Ophthalmol 148, 6 (2009), 844-851.

[45] Dorronsoro, C., Pascual, D., Perez-Merino, P., Kling, S., AND MARCos, S. Dynamic oct mesurement of corneal deformation by air-puff in normal and cross-linked corneas. Biomed Opt Exp 3, 3 (2012), 473-487.

[46] Downs, J., Suh, J., Thomas, K., Bellezza, A., Hart, R., And Burgoyne, C. Viscoelastic material properties of the peripapillary sclera in normal and early-glaucoma monkey eyes. Invest Ophth Vis Sci 46, 2 (2005), 540-546.

[47] Dua, H., Faraj, L., Said, D., Gray, T., AND Lowe, J. Human corneal anatomy redefined: A novel pre-descemet's layer (dua's layer). Ophthalmology 120, 9 (2013), 1778-1785.

[48] Dubbelman, M., Weeber, H., van der Heijde, R., ANd VolKerDieben, H. Radius and asphericity of the posterior corneal surface determined by corrected scheimpflug photography. Acta Ophthalmol. Scand. 80, 4 (2002), 379-383. 
[49] Dupps, W. Biomechanical modeling of corneal ectasia. Journal of Refractive Surgery 21 (2005), 186-190.

[50] Dupps, W., AND Wilson, S. Biomechanics and wound healing in the cornea. Exp Eye Res 83 (2006), 709-720.

[51] Edelhauser, H. The balance between corneal transparancy and edema the procor lecture. Invest Ophth Vis Sci 47, 5 (2006), 1755-1767.

[52] Edmund, C. Corneal elasticity and ocular rigidity in normal and keratoconic eyes. Acta Ophthalmologica 66, 2 (1988), 130-140.

[53] Elsheikh, A., AND Alhasso, D. Mechanical anisotropy of porcine cornea and correlation with stromal microstructure. Exp Eye Res 88, 6 (2009), 1084-1091.

[54] Elsheikh, A., Alhasso, D., And Rama, P. Assessment of the epithelium's contribution to corneal biomechanics. Exp Eye Res 86 (2008), 445-451.

[55] Elsheikh, A., Alhasso, D., And Rama, P. Biomechanical properties of human and porcine corneas. Exp Eye Res 86 (2008), 783-790.

[56] Elsheikh, A., AND Anderson, K. Comparative study of corneal strip and extensometry and inflation tests. $J R$ Soc Interface 46, 2 (2005), 409-414.

[57] Elsheikh, A., Geraghty, B., Rama, P., CAmpanelli, M., And MEEK, K. Characterization of age-related variation in corneal biomechanical properties. J. R. Soc. Interface 7, 51 (2010), 14751485.

[58] Elsheikh, A., KAssem, W., AND Jones, W. Strain-rate sensitivity of porcine and ovine corneas. Acta of 291 Bioengineering and Biomechanics 13, 2 (2011), 25-36. 
[59] Elsheikh, A., Wang, D., Brown, M., Rama, P., Campanelli, M., AND PYE, D. Assessment of corneal biomechanical properties and their variation with age. Current Eye Research 1, 32 (2007), 11-19.

[60] ERTAN, A., AND Colin, J. Intracorneal rings for keratoconus and keratectasia. J Cataract Refract Surg 33, 7 (2007), 1303-1314.

[61] EWINs, D. Modal Testing: theory, practice and application (2nd Ed.). Wiley, 2000.

[62] Ferrara, G., Torquetti, L., Ferrara, P., and Merayo-Lloves, J. Intrastromal corneal ring segments: visual outcomes from a large case series. Clin Exp Ophthalmol 40 (2012), 433-439.

[63] Fink, A., Gore, C., And Rosen, E. Corneal changes associated with intrastromal corneal ring segments. Archives of Ophthalmology 117 (1999), 282.

[64] Flemming, J., Wan, W., And Schanzlin, D. The theory of corneal curvature change with the intrastromal corneal ring. CLAO J 15 (1989), 146-150.

[65] Fleury, V., Al-Kilani, A., Boryskina, O., Cornelissen, A., Nguyen, T., Unbekandt, M., Leroy, L., Baffet, G., Le Noble, F., Sire, O., Lahaye, E., ANd Burgaud, V. Introducing the scanning air puff tonometer for biological studies. Physical Review E 81 (2010), 021920-1-15.

[66] Fontes, B., Ambrosio, R., Jardim, D., Velarde, G., And Nose, W. Corneal biomechanical metrics and anterior segment parameters in mild keratoconus. Ophthalmology 117 (2011), 673-679.

[67] Fontes, B., Ambrosio, R., Velarde, G., And Nose, W. Ocular response analyzer measurements in keratoconus with normal 
central corneal thickness compared with matched normal control eyes. Journal of Refractive Surgery 27 (2011), 209-215.

[68] Ford, M., Dupps, W., Rollins, A., Roy, A., AND Hu, Z. Method for optical coherence elastography of the cornea. Journal of Biomedical Optics 16 (2011).

[69] Fratzl, P., AND DAXER, A. Structural transformation of collagen fibrils in corneal stroma during drying: An x-ray scattering study. Biophys J 64 (1993), 1210-1214.

[70] FRIEDEnWALD, J. Contribution to the theory and practice of tonometry. Am J Ophthalmol 20 (1937), 985-1024.

[71] Garcia-Ramos, F., Valero, C., Homer, I., Ortiz-Canavate, J., AND Ruiz-Altisent, M. Non-destructive fruit firmness sensors: a review. Spanish Journal of Agricultural Research 3, 1 (2005), 61-73.

[72] Gefen, A., Shalom, R. An Elad, D., And Mandel, Y. Biomechanical analysis of the keratoconic cornea. J Mech Behav Biomed Mater 2 (2009), 224-236.

[73] Girard, M., Suh, J.-K., Bottlang, M., Burgoyne, C., And Downs, J. Biomechanical changes in the sclera of monkey eyes exposed to chronic iop elevations. Invest Ophthalmol Vis Sci 52 (2011), 5656-5669.

[74] Glass, D., Roberts, C., Litsky, A., AND Weber, P. A viscoelastic biomechanical model of the cornea describing the effect of viscosity and elasticity on hysteresis. Invest Ophthalmol Vis Sci 49 (2008), 3919-3926.

[75] Gora, M., Karnowski, K., Szkulmowski, M., Kaluzny, B., Huber, R., KowAlczYK, A., AND WojtKowski, M. Ultra highspeed swept source oct imaging of the anterior segment of human 
eye at $200 \mathrm{khz}$ with adjustable imaging range. Opt. Express 17, 17 (2009), 14880-14894.

[76] Grabner, G., Eilmsteiner, R., Steindl, C., Ruckhofer, J., MATtioli, R., AND W., H. Dynamic corneal imaging. Journal of Cataract and Refractive Surgery 31 (2005), 163-174.

[77] Grulkowski, I., Gora, M., Szkulmowski, M., Gorczynska, I., SzlaG, D., Marcos, S., KowalczyK, A., AND WoJTKowski, M. Anterior segment imaging with spectral oct system using a high-speed cmos camera. Opt Express 17, 6 (2009), 4842-4858.

[78] Guthoff, R., Zhivov, A., AND O., S. In vivo confocal microscopy, an inner vision of the cornea - a major review. Clinical and Experimental Ophthalmology 37 (2009), 100-117.

[79] Hafezi, F., Kanellopoulos, J., Wiltfang, R., And Seiler, T. Corneal collagen crosslinking with riboflavin and ultraviolet a to treat induced keratectasia after laser in situ keratomileusis. $J$ Cataract Rafr Surg 33 (2007), 2035-2040.

[80] Hafezi, F., Mrochen, M., Iseli, H., And Seiler, T. Collagen crosslinking with ultraviolet-a and hypoosmolar riboflavin solution in thin corneas. Journal of Cataract and Refractive Surgery 35 (2009), 621-624.

[81] Hamaoui, M., Tahi, H., Chapon, P., Duchesne, B., Fantes, F., FEuER, W., AND JEAN-MARIE. Corneal preparation of eye bank eyes for experimental surgery. Cornea 20, 3 (2010), 317-320.

[82] Hamilton, K., AND PYE, D. Youngs modulus in normal corneas and the effect on applanation tonometry. Optom Vis Sci 85 (2008), 445-450.

[83] Han, Z., Sui, X., Zhou, D., Zhou, C., And Ren, Q. Biomechanical and refractive behaviors of keratoconic cornea based on 
three-dimensional anisotropic hyperelastic models. J Refract Surg 29, 4 (2013), 282-290.

[84] Hayes, S., Boote, C., Lewis, J., AND et Al. Comparative study of fibrillar collagen arrangement in the corneas of primates and other mammals. Anat Rec 290, 12 (2007), 1542-1550.

[85] HE, X., AND LIU, J. A quantitative ultrasonic spectroscopy method for noninvasive determination of corneal biomechanical properties. Invest. Ophthalmol. Vis. Sci. 50 (2009), 5148-5154.

[86] Hennighausen, H., Feldman, S., Bille, J., And McCulloch, A. Anterior-posterior strain variation in normally hydrated and swollen rabbit cornea. Invest Ophth Vis Sci 39, 2 (1998), 253-262.

[87] HJortdal, J. Extensibility of the normo hydrated human cornea. Acta Opthalmologica 73, 1 (1995), 12-17.

[88] HJORTDAL, J. Regional elastic performance of the human cornea. J Biomech 29, 7 (1996), 931-942.

[89] HJortdal, J., AND Koch Jensen, P. In vitro measurement of corneal strain, thickness and curvature using digital image processing. Acta Opthalmologica 73 (1995), 5-11.

[90] Hoeltzel, D., Altman, P., Buzard, K., And Choe, K. Strip extensiometry for comparison of the mechanical response of bovine, rabbit and human corneas. J Biomech Eng 114, 2 (1992), 202-215.

[91] Holopainen, J., And Krootila, K. Transient corneal thinning in eyes undergoing corneal cross-linking. American Journal of Ophthalmology 152 (2011), 533-536.

[92] Holzer, M., Mannsfeld, A., Ehmer, A., And Auffarth, G. Early outcomes of intracor femtosecond laser treatment for presbyopia. J. Refract. Surg. 25, 10 (2009), 855-861. 
[93] Hon, Y., AND LAM, A. Corneal deformation measurement using scheimpflug noncontact tonometry. Optometry and Vision Science 90, 1 (2013), 1-8.

[94] Hong, J., Xu, J., Wei, A., Deng, S., Cui, X., Yu, X., AND Sun, $\mathrm{X}$. A new tonometer - the corvis st tonometer clinical comparison with non-contact, and goldmann applanation tonometers. Invest Ophthalmol Vis Sci, in press (2013), iovs.12-10984.

[95] Hull, D., Green, K., And Bowman, K. Dextran uptake into and loss from corneas stored in intermediate-term preservative. Invest Ophth Visual (1976), 663-666.

[96] Iribarren, R., Morgan, I., NAngia, V., And Jonas, J. Crystalline lens power and refractive error. Invest Ophthalml Vis Sci 53, 2 (2011), 543-550.

[97] IZATt, J., AND Choma, M. chap. 2, Optical Coherence Tomography Technology and Applications, theory of optical coherence tomography ed., vol. XXIX. Springer, 2008.

[98] Izatt, J., Hee, M., Swanson, E., Lin, C., Huang, D., Schuman, J., Puliafito, C., ANd Fujimoto, J. Micrometer-scale resolution imaging of the anterior eye in vivo with optical coherence tomography. Arch. Ophthalmol. 112, 12 (1994), 1584-1589.

[99] Jayasuriya, A., Scheinbeim, J., Lubkin, V., Bennett, G., AND KrAMER, P. Piezoelectric and mechanical properties in bovine cornea. Journal of Biomedical Materials Research Part A 66A (2003), 260-265.

[100] Johnson, C., Mian, S., Moroi, S., Epstein, D., IzATt, J., And AFSHARI, N. Role of corneal elasticity in damping of intraocular pressure. Invest Ophthalmol Vis Sci 48, 6 (2007), 2540-2544.

[101] Jue, B., AND MAURICE, D. The mechanical properties of the rabbit and human cornea. J Biomech 19, 10 (1986), 847-853. 
[102] Kamaev, P., Friedman, M., Sherr, E., And Muller, D. Photochemical kinetics of corneal cross-linking with riboflavin. Invest Ophthalmol Vis Sci 53, 4 (2012), 2360-2367.

[103] KAMpMEIER, J., RADT, B., BiRngRuber, R., AND BRINKMANn, R. Thermal and biomechanical parameters of porcine cornea. Cornea 19 (2000), 355-363.

[104] Kanellopoulos, A. Comparison of sequential vs same-day simultaneous collagen cross-linking and topography-guided prk for treatment of keratoconus. J Refract Surg 25, 9 (2009), 812-819.

[105] KikKaWA, Y., AND Hirayama, K. Uneven swelling of corneal stroma. Investigative Ophthalmology 9 (1970), 735-xxx.

[106] Kissner, A., AND ET AL. Pharmacological modification of the epithelial permeability by benzalkonium chloride in uva/riboflavin corneal collagen cross-linking. Current Eye Research 35 (2010), 715-721.

[107] Kling, S., Ginis, H., ANd Marcos, S. Corneal biomechanical properties from two-dimensional corneal flap extensiometry: Application to uv-riboflavin cross-linking. Invest. Ophthalmol. Vis. Sci. 53 (2012), 5010-5015.

[108] KLING, S., AND MARCos, S. Effect of hydration state and storage media on corneal biomechanical response from in-vitro inflation tests. Journal of Refractive Surgery 29, 7 (2013), 490-497.

[109] Kling, S., Remon, L., Pz-Escudero, A., Lloves-Merayo, J., AND MARCos, S. Corneal biomechanical changes after collagen cross-linking from porcine eye inflation experiments. Invest Ophth Vis Sci 51, 8 (2010), 3961-3968.

[110] KLING, S., AND S., M. Contributing factors to corneal deformation in air puff measurements. Invest Ophthalmol Vis Sci 54, 7 (2013), 5078-5085. 


\section{BIBLIOGRAPHY}

[111] Kniestedt, C., Nee, M., And Stamper, R. Accuracy of dynamic contour tonometry compared with applanation tonometry in human cadaver eyes of different hydration states. Graefes Arch Clin Exp Ophthalmol 243 (2005), 359-366.

[112] Knox Cartwright, N., Tyrer, J., And Marshal, L. J. Agerelated differences in the elasticity of human cornea. Invest Invest Ophthalm Vis Sci 52, 7 (2011), 4324-4329.

[113] KohlhaAs, M., Spoerl, E., Schilde, T., Unger, G., Wittig, C., AND Pillunat, L. Biomechanical evidence of the distribution of cross-links in corneastreated with riboflavin and ultraviolet a light. J Cataract Refract Surg 32, 2 (2006), 279-283.

[114] Kontadakis, G., Ginis, H., Karyotakis, N., Pennos, A., PenTARI, I., KYMIONIS, G., AND PALLIKARIS, I. The effect of riboflavin mediated corneal crosslinking on corneal hydration. Acta Opthalmologica 88, s246 (2010).

[115] Koppen, C., Wouters, K., Mathysen, D., Rozema, J., And TasSIGNON, M.-J. Refractive and topographic results of benzalkonium chloride-assisted transepithelial crosslinking. Journal of Cataract and Refractive Surgery 38 (2012), 1000-1005.

[116] Krachmer, J., Feder, R., ANd Belin, M. Keratoconus and related noninflammatory corneal thinning disorders. Survey of Ophthalmology 28, 4 (1984), 293-322.

[117] Krachmer, J. H., Mannis, M. J., And Holland, E. J. Cornea: Fundamentals, Diagnosis and Management. Elsevier MOSBY, 2005.

[118] Kubaloglu, A., Cinar, Y., Sari, E., Koytak, A., Ozdemir, B., AND OZERTURK, Y. Comparison of 2 intrastromal corneal ring segment models in the management of keratoconus. $J$ Cataract Refract Surg 36 (2010), 978-985. 
[119] KYMIOnis, G., AND ET, A. Intraoperative pachymetric measurements during corneal collagen cross-linking with riboflavin and ultraviolet a irradiation. Ophthalmology 116 (2009), 2336-2339.

[120] KYMionis, G., AND ET AL. Customized pachymetric guided epithelial debridement for corneal collagen cross linking. Bmc Ophthalmology 9 (2009).

[121] Kymionis, G., Kounis, G., Portaliou, D., And ET AL. Intraoperative pachymetry measurements during corneal collagen crosslinking with riboflavin and ultraviolet a irradiation. Ophthalmology 116, 12 (2009), 2336-2339.

[122] LeE, Y., Owens, C., And Meullenet, J. A novel laser air puff and shape profile method for predicting tenderness of broiler breast meat. Poult Sci 87, 7 (2008), 1451-1457.

[123] Litwiller, D., Lee, S., KolipakA, A., AND ET Al. Mr elastography of the ex vivo bovine globe. J Magn Reson Imaging 32 (2010), 44-51.

[124] LiU, Z., AND YEUnG, K. The preconditioning and stress relaxation of skin tissue. Journal of Biomedical Pharmaceutical Engineering 2, 1 (2008), 22-28.

[125] LUCE, D. Determining in vivo biomechanical properties of the cornea with an ocular response analyzer. $J$ Cataract Refract Surg 31 (2005), 156-162.

[126] Maeda, N., Fujikado, T., Kuroda, T., And et Al. Wavefront aberrations measured with hartmann-shack sensor in patients with keratoconus. Ophthalmology 109 (2002), 1996-2003.

[127] Malik, S., Moss, S., Ahmed, N., Furth, A., Wall, R., AND MEEK, K. Ageing of the human corneal stroma: structural and biochemical changes. Biochimica et Biophsica Acta - Molecular Basis of Disease 1138, 3 (1992), 222-228. 
[128] Maurice, D. M. The cornea and the sclera. The Eye 13 (1984), 1-158.

[129] McNeill, R. Tendon elasticity and muscle function. Comparative Biochemistry and Physiology Part A: Molecular Integrative Physiology 133, 4 (2002), 1001 4ww1.

[130] Meek, K., AND Boote, C. Te use of x-ray scattering techniques to quantify the orientation and distribution of collagen in the corneal stroma. Porgress in Retinal and Eye Research 28 (2009), 369-392.

[131] Meek, K., And Newton, R. Organization of collagen fibrils in the corneal stroma in relation to mechanical properties and surgical practice. J Refract Surg 15, 6 (1999), 695-699.

[132] Meek, K., Tuft, S., Huang, Y., And ET AL. Changes in collagen orientation and distribution in keratoconus corneas. Invest Ophthalmol Vis Sci 46 (2005), 1948-1956.

[133] Metzler, K., Roberts, C., Whitaker, S., Lawrence, M., MaLIK, J., AND BONS, J. Modelling corneal response to an air puff using deformation data to retrieve young's modulus. Investigative Ophthalmology and Vision Science 237, E (2013), 1629.

[134] Miles, C., Avery, N., Rodin, V., And A.J., B. The increase in denaturation temperature following cross-linking is caused by dehydration of the fibres. J Mol Biol 346 (2005), 551-556.

[135] Miranda, D., Sartori, M., Francesconi, C., Allemann, N., FERRARA, P., AND CAMPOS, M. Ferrara intrastromal corneal ring segments for severe keratoconus. J Refractive Surg 19 (2003), $645-653$.

[136] Morgan, S., Dooley, E., Hocking, P., Ingleheam, C., Ali, M., Sorensen, T., Meek, K., AND Boote, C. An x-ray scattering study into the structural basis of corneal refractive function in an avian model. Biophysical Journal 104 (2013), 2586-2594. 
[137] Morishige, N., Wahlert, A., Kenney, M., And et AL. Secondharmonic imaging microscopy of normal human and keratoconus cornea. Invest Ophthalmol Vis Sci 48 (2007), 1087-1094.

[138] Muthupillai, R., Lomas, D., Rossman, P., Greenleaf, J., ManDUCA, A., AND EHMAN, R. Magnetic resonance elastography by direct visualization of propagating acoustic strain waves. Science 269, 5232 (1995), 1854-1857.

[139] Nakayasu, K., Tanaka, M., Konomi, H., And Hayashi, T. Distribution of types i, ii, iii, iv and v collagen in normal and keratoconus corneas. Ophthalmic Res 18 (1986), 1-10.

[140] Nash, I., Greene, P., And Foster, C. Comparison of mechanical properties of keratoconus and normal corneas. Exp Eye Res 35 (1982), 413-424.

[141] NAvarro, R. The optical design of the human eye: a critical review. Journal of Optometry 2, 1 (2009), 3-18.

[142] Nichols, J., Marsich, M., Nguyen, M., Barr, J., ANd BulLIMORE, M. Overnight orthokeratology. Optom. Vis. Sci. 77, 5 (2000), 252-259.

[143] Nicolle, S., AND Palierne, J. Dehydration effect on the mechanical behaviour of biological soft tissues: Observations on kidney tissues. Journal of the Mechanical Behavior of Biomedical Materials 3 (2010), 630-635.

[144] Olsen, T., Sanderson, S., Feng, S., and Hubbard, W. Porcine sclera: Thickness and surface area. Invest Ophthal Vis Sci 43, 8 (2002), 2529-2532.

[145] Ortiz, D., Pinero, D., Shabayek, M., Arnalich-Montiel, F., AND ALIO, J. Corneal biomechanical properties in normal, postlaser in situ keratomileusis and keratoconic eyes. $J$ Cataract Refr Surg 33, 8 (2007), 1371-1375. 
[146] ORTIZ, S., AND ET AL. Optical distortion correction in optical coherence tomography for quantitative ocular anterior segment by three-dimensional imaging. Optics Express 18 (2010), 2782-2796.

[147] Ortiz, S., Perez-Merino, P., Alejandre, N., Gambra, E., Jimenez-Alfaro, I., AND MArcos, S. Quantitative oct-based corneal topography in keratoconus with intracorneal ring segments. Biomed Opt Express 3 (2012), 814-824.

[148] Ortiz, S., Siedlecki, D., Perez-Merino, P., Chia, N., De CasTro, A., SzKulmowski, M., WoJkowski, M., AND MARcos, S. Corneal topography from spectral optical coherence tomography (soct). Opt Express 2, 12 (2011), 3232-3247.

[149] Ortiz, S., Siedlecki, D., Remon, L., And Marcos, S. Optical coherence tomography for quantitative surface topography. Appl Optics 48 (2009), 6708-6715.

[150] Oxlund, H., AND Simonsen, A. Biochemical studies of normal and keratoconus corneas. Acta Opthalmologica 63, 6 (1985), 666-669.

[151] Pallikaris, I., Kymionis, G., Ginis, H., Kounis, G., And TsilIMBARIS, M. Ocular rigidity in living human eyes. Invest Ophth Vis Sci 46, 2 (2005), 409-414.

[152] Pandolfi, A., And Manganiello, F. A model for the human cornea: constitutive formulation and numerical analysis. Biomech Model Mechanobiol 5 (2006), 237-246.

[153] Patel, S., Marshall, J., And Fritzke, F. Iii. model for deriving the optical performance of the myopic eye corrected with an intracorneal ring. J Refract Surg 11 (1995), 248-252.

[154] Perez-Escudero, A., Dorronsoro, C., Sawides, L., Rem., Merayo-Lloves, J., AND Marcos, S. Minor influence of myopic 
laser in situ keratomileusis on the posterior corneal surface. Invest Ophth Vis Sci 50, 9 (2009), 4146-4154.

[155] Perez-Escudero, A., Dorronsoro, C., Sawides, L., Remon, L., Merayo-Lloves, J., AND Marcos, S. Minor influence of myopic laser in situ keratomileusis on the posterior corneal surface. Invest Ophthalmol Vis Sci 50, 9 (2009), 4146-4154.

[156] Perez-Merino, P., Parra, F., Ibares-Fras, L., AND et Al. Clinical and pathological effects of different acrylic intracorneal ring segments in corneal additive surgery. Acta Biomaterialia 6 (2012), 2572-2579.

[157] Petsche, S., AND PINSKY, P. The role of 3-d collagen organization in stromal elasticity: a model based on x-ray diffraction data and second harmonic-generated images. Biomech Model Mechanobiol $x x$ (2013), 1-13.

[158] Pierscionek, B., Asejczyk-Widlicka, M., And Schachar, R. The effect of changing intraocular pressure on the corneal and sclera curvatures in the fresh porcine eye. Br J Ophthalmol 91 (2007), 801-803.

[159] Pinero, D., Alio, J., El Kady, B., And Pascual, I. Corneal aberrometric and refractive performance of 2 intrastromal corneal ring segment models in early and moderate ectatic disease. $J$ Cataract Refract Surg 36 (2010), 102-109.

[160] Pinero, D., Alio, J., Teus, M., Barraquer, R., And UcedaMontannes, A. Modeling the intracorneal ring segment effect in keratoconus using refractive, keratometric, and corneal aberrometric data. Invest Ophthalmol Vis Sci 51 (2010), 5583-5591.

[161] Pinsky, P., AND DATYE, D. A microstructurally-based finite element model of the incised human cornea. J Biomech 24 (1991), 907-922. 


\section{BIBLIOGRAPHY}

[162] Pinsky, P., van Der Heide, D., And Chernyak, D. Computational modeling of mechanical anisotropy in the cornea and sclera. $J$ Cataract Refract Surg 31 (2005), 136-145.

[163] Rabinowitz, Y. Keratoconus. Surv Ophthalmol 42 (1998), 297319.

[164] Raiskup, F., Pinelli, R., ANd Spoerl, E. Riboflavin osmolar modification for transepithelial corneal cross-linking. Current Eye Research 37 (2012), 234-238.

[165] Raiskup-Wolf, F., Hoyer, A., Spoerl, E., And Pillunat, L. Collagen crosslinking with riboflavin and ultraviolet-a light in keratoconus: Long-term results. Journal of Cataract and Refractive Surgery 34 (2008), 796-801.

[166] Recep, Ó.F. AbD Hasiripi, H., Nurullah, C., AND SRIKatiPOGLU, H. Relation between corneal thickness and intraocular pressure measurement by noncontact and applanation tonometry. J Cataract Refract Surg 27, 11 (2001), 1787-1791.

[167] Ritz, W. Uber eine neue metode zur losung gewisser variationsprobleme der matematischen physik. J. Reine Angew. Math. 135 (1909), 1-61.

[168] Roberts, C. The cornea is not a piece of plastic. $J$ Refract Surg 16, 4 (2000), 407-413.

[169] Rosales, P., AND MARcos, S. Quantitative scheimpflug imaging of the crystalline and intraocular lens. J Refract Surg 25, 5 (2009), 421-428.

[170] RoY, A., AND DuPPs, W. J. Patient-specific computational modeling of keratoconus progression and differential response to collagen cross-linking. Invest Ophthalmol Vis Sci 52 (2011), 9174-9187. 
[171] Ruckhofer, J., Stoiber, J., Twa, M., And Grabner, G. Correction of astigmatism with short arc-length intrastromal corneal ring segments: preliminary results. Ophthalmology 110 (2003), $516-524$.

[172] Ruiz-Ederra, J., Garcia, M., Hernandez, M., Urcola, H., Hernandez-Barbachano, E., Araiz, J., and Vecino, E. The pig eye as a novel model of glaucoma. Exp Eye Res 81, 5 (2005), 561-569.

[173] Sarvazyan, A., Rudenko, O., Swanson, S., Fowlkes, J., AND EMELIANOV, S. Shear wave elasticity imaging: A new ultrasonic technology of medical diagnosis. Ultrasound in Medicine and Biology 24, 9 (1998), 1419-1435.

[174] ScARCElli, G., Kim, P., AND Yun, S. Cross-axis cascading of spectral dispersion. Optics Letters 33 (2008), 2979-2981.

[175] SCARCELli, G., KiM, P., AND YUn, S. In vivo measurement of age-related stiffening in the crystalline lens by brillouin optical microscopy. Biophysical Journal 101 (2011), 1539-1545.

[176] Scarcelli, G., Kling, S., Quijano, E., Pineda, R., Marcos, S., AND YUN, S. Brillouin microscopy of collagen crosslinking: noncontact depth-dependent analysis of corneal elastic modulus. Invest Ophthalmol Vis Sci 54, 2 (2013), 1418-1425.

[177] ScArcelli, G., Pineda, R., ANd Yun, S. Brillouin optical microscopy for corneal biomechancis. Invest Ophthalmol Vis Sci 53, 1 (2012), 185-190.

[178] ScArcelli, G., AND Yun, S. Confocal brillouin microscopy for three-dimensional mechanical imaging. Nature Photonics 9, 2 (2007), 39-43. 


\section{BIBLIOGRAPHY}

[179] SCARCELli, G., AND Yun, S. Multistage vipa etalons for highextinction parallel brillouin spectroscopy. Optics Express 19 (2011), 10913-10922.

[180] SCARCELLI, G., AND Yun, S. In vivo brillouin optical microscopy of the human eye. Optics Express 20 (2012), 9197.

[181] Schanzlin, D., Asbell, P., Buris, T., And Durrie, D. The intrastromal corneal ring segments. phase ii results for the correction of myopia. Ophthalmology 104 (1997), 1067-1078.

[182] Schumacher, S., Mrochen, M., Wernli, J., Bueeler, M., AND SEILER, T. Optimization model for uv-riboflavin corneal crosslinking. Invest. Ophthalmol. Vis. Sci. 53 (2012), 762-769.

[183] Sedaghat, M., NAderi, M., And Zarei-Ghanavati, M. Biomechanical parameters of the cornea after collagen crosslinking measured by waveform analysis. J Cataract Refract Surg 36 (2010), 1728-1731.

[184] SeIler, T., AND HAfEzi, F. Corneal cross-linking-induced stromal demarcation line. Cornea, 25 (2006), 10571059.

[185] Shah, S., Laiquzzaman, M., Bhojwani, R., Mantry, S., AND Cunliffe, I. Assessment of the biomechanical properties of the cornea with the ocular response analyzer in normal and keratoconic eyes. Invest. Ophthalmol. Vis. Sci. 48 (2007), 30263031.

[186] SHIELDS, B. The non-contact tonometer. its value and limitations. Survey of Ophthalmology 24, 4 (1980), 211-219.

[187] Shin, T., Vito, R., Johnson, L., And McCarey, B. The distribution of strain in the human cornea. J Biomech 30, 5 (1997), 497-503.

[188] Siepser, S., AND inventor Kera Associates, A. Device used to change corneal curvature, 1990 . US patent 4,976,719. 
[189] Siganos, C., Kymionis, G., Kartakis, N., Theodorakis, M., ASTYRAKAKIS, N., AND PALLIKARIS, I. Management of keratoconus with intacs. Am J Ophthalmol 135 (2003), 64-70.

[190] Siganos, D., Ferrara, P., Chatyinikolas, K., Bessis, N., AND PAPASTERgioU, G. Ferrara intrastromal corneal rings from the correction of keratoconus. J Cataract Refr Surg 28, 11 (2002), 1947-1951.

[191] Silvestrini, T., Mathis, M., Loomas, B., And Burris, T. A geometric model to predict the change in corneal curvature from the intrastromal corneal ring (icr). Invest Ophthalmol Vis Sci 35(suppl) (1994), 2023.

[192] Simon, G., Parel, J., Lee, W., And Kervick, G. Gel injection adjustable keratoplasty. Graefes Arch Clin Exp Ophthalmol 229 (1991), 418-424.

[193] SondergaARd, A., HJortdal, J., Breitenbach, T., AND IVARSEN, A. Corneal distribution of riboflavin prior to collagen cross-linking. Current Eye Research 35 (2010), 116-121.

[194] Spoerl, E., Huhle, M., Kasper, M., And Seiler, T. Erhhung der festigkeit der hornhaut durch vernetzung. Ophthalmologe 94 (1997), 902-906.

[195] Spoerl, E., Huhle, M., AND SeIler, T. Induction of cross-links in corneal tissue. Exp Eye Res 66, 1 (1998), 97-103.

[196] Spoerl, E., Mrochen, M., Sliney, D., Trokel, S., ANd Seiler, T. Safety of uv-a riboflavin cross-linking of the cornea. Cornea 26, 4 (2007).

[197] Spoerl, E., Raiskup-Wolf, F., And Pillunat, L. Biophysical principles of collagen crosslinking. Klinische Monatsblatter Fur Augenheilkunde 225 (2008), 131-137. 


\section{BIBLIOGRAPHY}

[198] Spoerl, E., Schreiber, J., Hellmund, K., AND et Al. Studies on the stabilization of the cornea in rabbits. Ophthalmologe 97, 3 (2000), 203-206.

[199] Spoerl, E., Terai, N., Scholz, F., Raiskup, F., And Pillunat, L. Detection of biomechanical changes after corneal cross-linking using ocular response analyzer software. Journal of Refractive Surgery 27 (2011), 452-457.

[200] SRODKA, W., AND IsKANDER, D. Optically inspired biomechanical model of the human eyeball. $J$ Biomed Opt 13, 4 (2008), 04403410440348.

[201] Stefancu, A.-I., Melenciuc, S.-C., And Budescu, M. Finite element analysis of frictional contacts. Bul Inst Politeh Iasi 58 (2011), 131-139.

[202] Steinert, R., And Huang, D. Anterior Segment Optical Coherence Tomography. SLACK Incorporated, Thorofare, N.J., 2008.

[203] Stodtmeister, R. Applanation tonometry and correction according to corneal thickness. Acta Ophthalmologica Scandinavica 76, 3 (1998), 319-324.

[204] Suri, K., Hammersmith, K., And Nagra, P. Corneal collagen cross-linking: ectasia and beyond. Current Opinion in Ophthalmology 23 (2012), 280-287.

[205] Swan, L., Gordon, R., AND SEcKBACH, J. Origin(s) of Design in Nature: A Fresh, Interdisciplinary Look at How Design Emerges in Complex Systems, Especially Life, vol. 23. Springer, 2012.

[206] TANTER, M. High-resolution quantitative imaging of cornea elasticity using supersonic shear imaging. Medical Imaging, IEEE Transactions 28, 12 (2009), 1881-1893. 
[207] Terai, N., Raiskup, F., Haustein, M., Pillunat, L., and Spoerl, E. Identification of biomechanical properties of the cornea: The ocular response analyzer. Curr Eye Res 37 (2012), 553-562.

[208] Tower, T., Neidert, M., AND Tranquillo, R. Fiber alignment imaging during mechanical testing of soft tissue. Ann of Biomed Eng 30 (2002), 1221-1233.

[209] Tsonis, P. A. Animal Models in Eye Research. Academic Press, 2008.

[210] Twa, M., Ruckhofer, J., Kash, R., Costello, M., And SchanZLIN, D. Histologic evaluation of corneal stroma in rabbits after intrastromal corneal ring implantation. Cornea 22 (2003), 146152.

[211] VAUghan, J., AND RANDALL, J. Brillouin scattering, density and elastic properties of the lens and cornea of the eye. Nature 284 (1980), 489-491.

[212] Vera-Diaz, F., AND Doble, N. The Human Eye and Adaptive Optics, Topics in Adaptive Optics, Dr. Bob Tyson (Ed.). InTech, 2012.

[213] Vinciguerra, P., Albe, E., Mahmoud, A., Trazza, S., Hafezi, F., AND ROBERTS, C. Intra- and postoperative variation in ocular response analyzer parameters in keratoconic eyes after corneal cross-linking. Journal of Refractive Surgery 9, 26 (2010), 669-676.

[214] Vinciguerra, P., Albe, E., Trazza, S., And et Al. Refractive, topographic, tomographic and aberrometric analysis of keratoconic eyes undergoing corneal cross-linking. Ophthalmology 116, 3 (2009), 369-378.

[215] Wang, H., Prendiville, P., McDonnell, P., And Chang, W. An ultrasonic technique for the measurement of the elastic moduli of human cornea. Journal of Biomechanics 29 (1996), 1633-1636. 
[216] Wang, S., Li, J., Manapuram, R., Menodiado, F., Ingram, D., Twa, M., Lazar, A., Lev, D., Pollock, R., And Larin, K. Noncontact measurement of elasticity for the detection of softtissue tumors using phase-sensitive optical coherence tomography combined with a focused air-puff system. Optics Letter 37, 24 (2012), 5184-5186.

[217] Wilson, G., Oleary, D., And Vaughan, W. Differential swelling in compartments of the corneal stroma. Invest. Ophthalmol. Vis. Sci. 25 (1984), 1105-1108.

[218] Wilson, S., Lin, D., AND Klyce, S. Corneal topography of keratoconus. Cornea 10 (1991), 2-8.

[219] Winkler, M., Chai, D., Krilling, S., Nien, C., Brown, D., Jester, B., JubAsz, T., AND Jester, J. Nonlinear optical macroscopic assessment of 3-d corneal collagen organization and axial biomechanics. Invest Ophthalmol Vis Sci, 52 (2011), 8818-8827.

[220] WitTig-Silva, C., AND ET AL. Randomized controlled trial of corneal collagen cross-linking in progressive keratoconus: Preliminary results. Journal of Refractive Surgery 24 (2008), S720-S725.

[221] Wollensak, G. Crosslinkining treatment of progressive keratoconus: new hope. Curr Opin Ophthalmol 17, 4 (2006), 356-360.

[222] Wollensak, G., Aurich, H., Pham, D., And Wirbelbauer, C. Hydration behavior of porcine cornea crosslinked with riboflavin and ultraviolet. A. J Cataract Refract Surg 33, 3 (2007), 516 -521.

[223] Wollensak, G., AND IOMdina, E. Biomechanical and histological changes after corneal crosslinking with and without epithelial debridement. J Cataract Ref Surg 35, 3 (2009), 540-546.

[224] WollensaK, G., AND IOMdina, E. Long-term biomechanical properties of rabbit cornea after photodynamic collagen crosslinking. Acta Ophthalmol 87, 1 (2009), 48-51. 
[225] Wollensak, G., Spoerl, E., AND Seiler, T. Riboflavin/ultraviolet-a-induced collagen crosslinking for the treatment of keratoconus. Am. J. Ophthalmol. 135, 5 (2003), 620-627.

[226] Wollensak, G., Spoerl, E., AND Seiler, T. Stress-strain measurements of human and porcine corneas after riboflavinultraviolet-a-induced cross-linking. J Cataract Refr Surg 29 (2003), 1780-1785.

[227] Wollensak, G., Spoerl, E., Wilsch, M., And Seiler, T. Keratocyte apoptosis after corneal collagen cross-linking using riboflavin/uv-a treatment. Cornea 23, 1 (2004), 43-49.

[228] Wollensak, G., Wilsch, M., Spoerl, E., And Seiler, T. Collagen fiber diameter in the rabbit cornea after collagen crosslinking by riboflavin/uva. Cornea 23, 5 (2004), 503-507.

[229] Woo, S., Kobayashi, A., Schlegel, W., and LaWrence, C. Nonlinear material properties of intact cornea and sclera. Exp Eye Res 14, 1 (1972), 29-39.

[230] Y., G., Barkana, Y., Morad, Y., Hartstein, M., Avni, I., AND ZADOK, D. Can we measure corneal biomechanical changes after collagen cross-linking in eyes with keratoconus? - a pilot study. Cornea 5, 28 (2009), 498-502.

[231] Yanjun, Z., JiAn, Y., Kun, H., Zhihui, L., And Xiuyun, L. A comparison of biomechanical properties between human and porcine cornea. Journal of Biomechanics 34, 4 (2001), 533-537.

[232] Yuen, L., Chan, C., AND Wachler, B. Effect of epithelial debridement in corneal collagen crosslinking therapy in porcine and human eyes. Journal of Cataract and Refractive Surgery 34 (2008), 1815-1816. 


\section{BIBLIOGRAPHY}

[233] Zeng, Y., YANG, J., HuAng, K., LeE, Z., AND LeE, X. A comparison of biomechanical properties between human and porcine cornea. J Biomech 34 (2001), 533-537.

[234] ZERnike, F. Beugungstheorie des schneidenverfahrens und seiner verbesserten form, der phasenkontrastmethode. Physica 1, 7-12 (1934), 689-704. 


\section{Índice General}

0 Prólogo

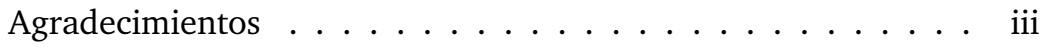

Motivación .................

Preguntas abiertas abordadas en ésta tesis . . . . . . . $\mathrm{v}$

Objetivos de ésta tésis . . . . . . . . . . . . . vii

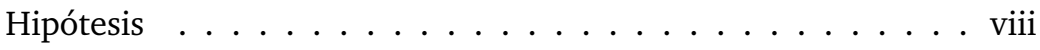

Estructura de esta tesis . . . . . . . . . . . . . . viii

1 Introducción 1

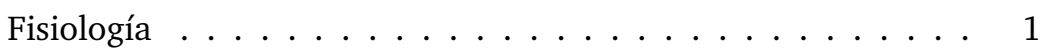

El ojo ........................ 1

La Cornea . . . . . . . . . . . . . . . . 3

Antecedentes en Óptica . . . . . . . . . . . . . . . . . . . . . 8

Frente de Onda . . . . . . . . . . . . 8

Aberraciones ................ 8

Errores refractivos del ojo . . . . . . . . . . . . . . . 9

Sistema de Trazado de Rayos . . . . . . . . . . . . 11

Coherencia . . . . . . . . . . . . . . 12

Interferencia . . . . . . . . . . . . . . . 13

Imagen del segmento anterior . . . . . . . . . . . . . . . . 13

Microscopía de la cornea . . . . . . . . . . . . . . . . . . . . . . . . . . 17

Enfermedades de la cornea y tratamientos . . . . . . . . . . . . . 21

Patologías de la cornea . . . . . . . . . . . . . . . . . . . . . . . . . 21

Tratamientos de la cornea . . . . . . . . . . . . . . 22 
Antecedentes mecánicos . . . . . . . . . . . . 26

Método de elementos finitos . . . . . . . . . . . . . . . . . 26

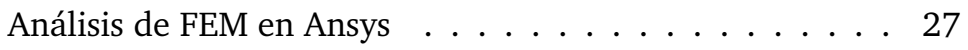

Modelos de material . . . . . . . . . . . . . . . . . . . . . . . . . . . . . . . .

Modelos de la cornea . . . . . . . . . . . . . . . . . . . . . . . . . . . . . . .

Pruebas de materiales . . . . . . . . . . . . . . . 36

Medida de la elasticidad de la cornea . . . . . . . . . . . . . . . . . . . . . . 40

Métodos in vitro . . . . . . . . . . . . . . . . . . . . . . . . . . 40

Métodos in vivo aplicados clínicamente . . . . . . . . . . . 42

Estado de la técnica de la biomecánica ocular experimental 45

2 Métodos 49

Métodos experimentales . . . . . . . . . . . . . . . . 51

Sistemas de Imagen . . . . . . . . . . . . . . . . . . . . . . . . . . . . . . . . .

Sistema de Presión . . . . . . . . . . . . . 60 60

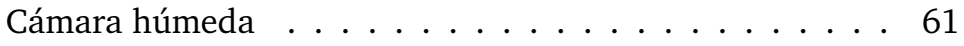

Porta ojos . . . . . . . . . . . . . . . . . . . 62

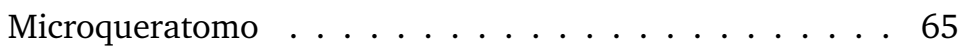

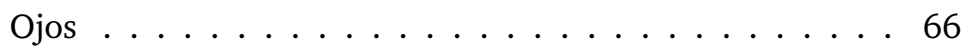

Almacenamiento . . . . . . . . . . . 666

Tratamientos de la cornea . . . . . . . . . . . . . . . . . . 67 67

Rutinas de análisis . . . . . . . . . . . . . . 69

Métodos teóricos . . . . . . . . . . . . 75

Software . . . . . . . . . . . . . . 75

Análisis refractivo de modelos FE . . . . . . . . . . 76

Modelos de elementos finitos . . . . . . . . . . . . . . . . . . . . . . . . . . . .

FEM Solver . . . . . . . . . . . . . . . 78

Análisis de senibilidad . . . . . . . . . . . . 78

3 Cambios biomecánicos tras cross-linking: Experimentos de inflado en ojos de cerdo $\quad 79$ Introducción $\ldots \ldots \ldots \ldots$. . . . . . . . . 81 
Protocolo .................... 83

Set-up . . . . . . . . . . . . . 8 84

Análisis de datos . . . . . . . . . . . . . . . 85

Resultados . . . . . . . . . . . . . . . 86

Cambios en el espesor corneal . . . . . . . . . . . . 86

Cambios en la posición del apex corneal . . . . . . . . . 87

Cambios en la curvatura corneal en el meridiano horizontal y vertical . . . . . . . . . . . . . . . . 990

Cambios refractivos: presión constante versus inflado . . . 94

Modelo biomecánico simple: Modulo de Young e histéresis 97 Discusión . . . . . . . . . . . . . . . 99

Comparación de datos experimentales con datos clínicos previos .................... 99

Implicaciones de los resultados . . . . . . . . . . . . 102

4 Efectos de la hidratación y medio de preservación sobre la respuesta biomecánica en experimentos de inflado en vitro 105 Introducción ． . . . . . . . . . . . . . . . 107 Condiciones y protocolos . . . . . . . . . . . . . . 108 Resultados . . . . . . . . . . . . . . . 110

Espesor corneal . . . . . . . . . . . . . . . 110

Curvatura de la cornea . . . . . . . . . . . . . . . . . 111

Hidratación de la cornea . . . . . . . . . . . . . . . . . 113

El epitelio y la transparencia de la cornea . . . . . . . . 113 Discusión . . . . . . . . . . . . . . . . . . 114

5 Propiedades biomecánicas de la cornea determinadas a partir de flaps bidimensionales aplicados a la evaluación de cross-linking 119 Introducción . . . . . . . . . . . . . . . . 121

Protocolo . . . . . . . . . . . . . . . . . 122

Ciclos de tensión y extensión . . . . . . . . . . . 124

Análisis de datos . . . . . . . . . . . . . . . . . 124 
Resultados . . . . . . . . . . . . . . . . 125

Paquimetría de los flaps y observaciones microscópicas . . 125

Aberración de desenfoque . . . . . . . . . . . . . . . . 125

Aberración de astigmatismo . . . . . . . . . . . 128

Módulo de Young . . . . . . . . . . . . . . . . . . . . . . . . . . . . . . . . . . . . . . . . . . . .

Discusión . . . . . . . . . . . . . . . . . 129

6 Microscopía de Brillouin aplicada a cross-linking: análisis sin contacto del módulo de elasticidad de la cornea en función de la profundidad

Introducción . . . . . . . . . . . . . 135

Protocolo . . . . . . . . . . . . . . 136

Reometría . . . . . . . . . . . . 138

Resultados . . . . . . . . . . . . . . . . 138

In situ caracterización de las propiedades mecánicas después de cross-linking . . . . . . . . . . . 138

Efecto de aplicación del fotosensibilizador y la duración de la exposición a la luz UV . . . . . . . . . . . 140

Cross-linking sin desepitelización . . . . . . . . . . . . . . . . . . . . . . . 142

Grupos de control . . . . . . . . . . . . . . . . . . 144

Efecto de hidratación sobre el modulo de Brillouin y de corte145

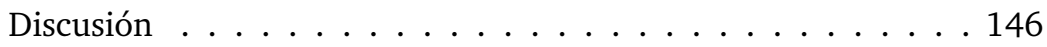

7 Determinación de propiedades biomecánicas de la cornea a partir de la deformación tras un pulso de aire 151 Introducción . . . . . . . . . . . . . . . . . . . . . . . . . . 153

Protocolo . . . . . . . . . . . . . . . 156

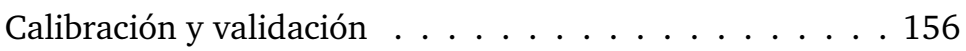

Ojos y mediciones . . . . . . . . . . . . . . . . . . . . . . . . . . . . . . . . . . . . . . . . . . .

Resultados . . . . . . . . . . . . . . . 158

Discusión . . . . . . . . . . . . . . . . . 159

Conclusiones ...................... 163 
8 Factores que contribuyen a la deformación corneal tras un pulso de aire Introducción . . . . . . . . . . . . . . . . 167

Condiciones y protocolos . . . . . . . . . . . . . . . . . . . . 169

Ojos de cerdo . . . . . . . . . . . . . . . . . . . . . . . 169

Ojos humanos . . . . . . . . . . . . . . . . . . 170

Diferentes IOPs (set de datos 1$) \ldots \ldots$. . . . . . . . 170

Espesor corneal y estado de hidratación (set de datos 1) 170

Rigidez de la cornea (set de datos 1) . . . . . . . . . . 171

Botones corneales versus globos enteros (set de datos 2) 171

in vitro versus in-vivo (set de datos 3 ) $\ldots \ldots \ldots 171$

Análisis . . . . . . . . . . . . . . . . . . . . . . . 172

Resultados . . . . . . . . . . . . . . . . . . . . 173

Efecto de la presión intraocular - set de datos $1 \ldots$. . . 173

Perfil de la deformación espacial . . . . . . . . . . . . . 174

Efecto de la deshidratación y de CXL - set de datos $1 \ldots 174$

Diferencias entre botón y globo entero - set de datos $2 \ldots 176$

Diferencias entre in vitro and in vivo - set de datos $3 \ldots 178$

Espesor central de la cornea - todos sets de datos . . . . . 178

Repetibilidad de las mediciones - set de datos $2 \ldots \ldots$. . 178

Discusión . . . . . . . . . . . . . . . . . . . 179

9 Evaluación no invasiva y sin contacto de las propiedades viscoelásticas de la cornea $\quad 185$ Introducción ． . . . . . . . . . . . . . . . . . 187

Simulación . . . . . . . . . . . . . . . . . . . . . . 190

Condiciones diferentes de la cornea . . . . . . . . . . . . 191

Diferentes presiones intraoculares (IOPs) . . . . . . . . . 191

Diferentes condiciones de contorno . . . . . . . . . . . 193

¿Qué determina la respuesta de la cornea? . . . . . . . . . 194

Discusión . . . . . . . . . . . . . . . . . . . . 195

Métodos . . . . . . . . . . . . . . . . . . . . . . . . . . . . 199

Imágenes de deformación con un pulso de aire . . . . . 199 
Sujetos y datos experimentales . . . . . . . . . . . . 199

Caraterización del pulso de aire . . . . . . . . . . . . . 199

Simulación con elementos finitos . . . . . . . . . . 200

Selección de parámetros . . . . . . . . . . . . . 202

Análisis . . . . . . . . . . . . . . . . 202

Técnicas de computación . . . . . . . . . . . . . . . 203

10 Vibrografía OCT para la cuantificación no invasiva de las propiedades biomecánicas de la cornea 205 Introducción _ . . . . . . . . . . . . . . . 207 Protocolo . . . . . . . . . . . . . . . . . . . . . . 208

Simulación con elementos finitos (FE) _ . . . . . . . 208

Experimentos . . . . . . . . . . . . . . 210

Resultados . . . . . . . . . . . . . . . . . . . . 212

Propiedades biomecánicas . . . . . . . . . . . . . . . 212

Flaps corneales . . . . . . . . . . . . . . . . . 212

Globos enteros . . . . . . . . . . . . . . . . . . 217

Discusión . . . . . . . . . . . . . . . . . . 223

11 Simulación con elementos finitos de la implantación de un anillo estromal en una cornea hiperelástica 227 Introducción . . . . . . . . . . . . . . . . . . . . . . 229 Protocolo . . . . . . . . . . . . . . . . . . . . 231

Simulación de la implantación de ICRS . . . . . . . . . . . 231

Modelado de ICRSs con elementos finitos . . . . . . . . 232

Descripcción del modelo . . . . . . . . . . . . . . . . 233

Simulación . . . . . . . . . . . . . . . . 233

Modelo de queratocono . . . . . . . . . . . . . . . 235

Resultados . . . . . . . . . . . . . . . . . . . . . 236

Cambios en el espesor corneal . . . . . . . . . . . . . . 237

Efecto de la geometría del ICRS sobre la curvatura anterior de la cornea . . . . . . . . . . . . . . . . 238

Cambios refractivos . . . . . . . . . . . . . . . . 241 
Cambios en la aberración esférica . . . . . . . . . . . . . 242

Efecto del tamaño del queratocono (modelo axisimétrico) 243

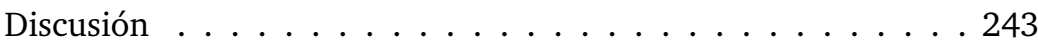

12 Conclusiones $\quad 249$

Logros . . . . . . . . . . . . . . . . . . . . 249

Conclusiones . . . . . . . . . . . . . 251

Trabajo futuro . . . . . . . . . . . . . 255

13 Publicaciones de esta tesis $\quad 257$

Publicaciones incluidas en ésta tesis . . . . . . . . . . . . . 257

Contribuciones a congrésos . . . . . . . . . . . . . 259

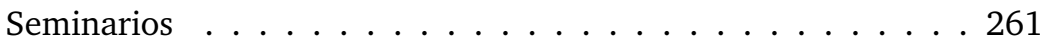

14 Resúmenes en Español 263

Capítulo $1 \ldots \ldots \ldots \ldots \ldots \ldots$

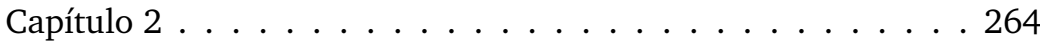

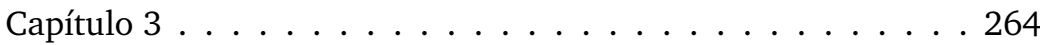

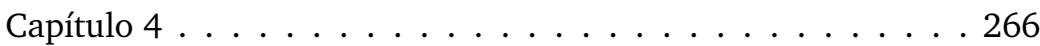

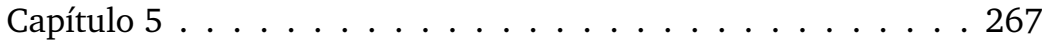

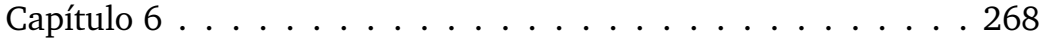

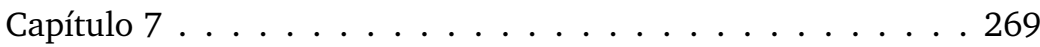

Capítulo $8 \ldots \ldots \ldots \ldots \ldots$. . . . . . . . . . . . . . . . . . . . .

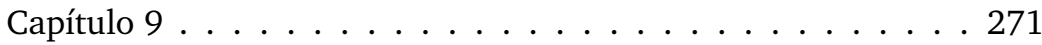

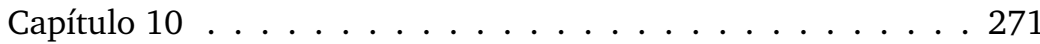

Capítulo 11 . . . . . . . . . . . . . . . . . 273

Conclusiones en Español . . . . . . . . . . . . . . . 274 
